Eastern Illinois University

The Keep

Faculty Research and Creative Activity

Psychology

$10-2013$

\title{
Making a stronger case for comparative research to investigate the behavioral and neurological bases of three-dimensional navigation
}

Daniele Nardi

Eastern Illinois University, dnardi@eiu.edu

Verner P. Bingman

Bowling Green State University - Main Campus

Follow this and additional works at: http://thekeep.eiu.edu/psych_fac

Part of the Psychology Commons

\section{Recommended Citation}

Nardi, Daniele and Bingman, Verner P., "Making a stronger case for comparative research to investigate the behavioral and neurological bases of three-dimensional navigation" (2013). Faculty Research and Creative Activity. 16.

http://thekeep.eiu.edu/psych_fac/16

This Article is brought to you for free and open access by the Psychology at The Keep. It has been accepted for inclusion in Faculty Research and Creative Activity by an authorized administrator of The Keep. For more information, please contact tabruns@eiu.edu. 


\title{
Navigating in a three-dimensional world
}

\author{
Kathryn J. Jeffery \\ Department of Cognitive, Perceptual and Brain Sciences, Division of \\ Psychology \& Language Sciences, University College London, London WC1H \\ $O A P$, United Kingdom \\ k.jeffery@ucl.ac.uk \\ www.ucl.ac.uk/jefferylab/ \\ Aleksandar Jovalekic ${ }^{1}$ \\ Institute of Neuroinformatics, University of Zurich, $\mathrm{CH}-8057$ Zurich, Switzerland \\ ajovalekic@ini.phys.ethz.ch \\ Madeleine Verriotis ${ }^{1}$ \\ Department of Neuroscience, Physiology and Pharmacology, University \\ College London, London WC1E 6BT, United Kingdom \\ madeleine.verriotis@ucl.ac.uk \\ Robin Hayman \\ Institute of Cognitive Neuroscience, Alexandra House, London WC1N 3AR, \\ United Kingdom \\ r.hayman@ucl.ac.uk
}

\begin{abstract}
The study of spatial cognition has provided considerable insight into how animals (including humans) navigate on the horizontal plane. However, the real world is three-dimensional, having a complex topography including both horizontal and vertical features, which presents additional challenges for representation and navigation. The present article reviews the emerging behavioral and neurobiological literature on spatial cognition in non-horizontal environments. We suggest that three-dimensional spaces are represented in a quasiplanar fashion, with space in the plane of locomotion being computed separately and represented differently from space in the orthogonal axis - a representational structure we have termed "bicoded." We argue that the mammalian spatial representation in surface-travelling animals comprises a mosaic of these locally planar fragments, rather than a fully integrated volumetric map. More generally, this may be true even for species that can move freely in all three dimensions, such as birds and fish. We outline the evidence supporting this view, together with the adaptive advantages of such a scheme.
\end{abstract}

Keywords: ethology; grid cells; head direction cells; hippocampus; navigation; neural encoding; place cells; spatial cognition; threedimensional

\section{Introduction}

Behavioural and neurobiological studies of spatial cognition have provided considerable insight into how animals (including humans) navigate through the world, establishing that a variety of strategies, subserved by different neural systems, can be used in order to plan and execute trajectories through large-scale, navigable space. This work, which has mostly been done in simplified, laboratory-based environments, has defined a number of sub-processes such as landmark recognition, heading determination, odometry (distance-measuring), and context recognition, all of which interact in the construction and use of spatial representations. The neural circuitry underlying these processes has in many cases been identified, and it now appears that the basics of navigation are reasonably well understood.

The real world, however, is neither simple nor twodimensional, and the addition of vertical space to the two horizontal dimensions adds a number of new problems for a navigator to solve. For one thing, moving against gravity imposes additional energy costs. Also, moving in a volumetric space is computationally complicated, because of the increased size of the representation needed, and because rotations in three dimensions interact. It remains an open question how the brain has solved these problems, and whether the same principles of spatial encoding operate in all three dimensions or whether the vertical dimension is treated differently.

Studies of how animals and humans navigate in environments with a vertical component are slowly proliferating, and it is timely to review the gathering evidence and offer a theoretical interpretation of the findings to date. In the first part of the article, we review the current literature on animal and human orientation and locomotion in three-dimensional spaces, highlighting key factors that contribute to and influence navigation in such spaces. The second part summarizes neurobiological studies of the encoding of three-dimensional space. The third and final part integrates the experimental evidence to put forward a hypothesis concerning three-dimensional spatial encoding-the bicoded model-in which we propose that in encoding 3D spaces, the mammalian brain constructs mosaics of connected surface-referenced 
maps. The advantages and limitations of such a quasi-planar encoding scheme are explored.

\section{Theoretical considerations}

Before reviewing the behavioural and neurobiological aspects of three-dimensional navigation it is useful to establish a theoretical framework for the discussion, which will be developed in more detail in the last part of this article. The study of navigation needs to be distinguished from the study of spatial perception per se, in which a subject may or may not be moving through the space. Additionally, it is also important to distinguish between space that is encoded egocentrically (i.e., relative to the subject) versus space that is encoded allocentrically (independently of the subject-i.e., relative to the world). The focus of the present article is on the allocentric encoding of the space that is being moved through, which probably relies on different (albeit interconnected) neural systems from the encoding of egocentric space.

An animal that is trying to navigate in $3 \mathrm{D}$ space needs to know three things - how it is positioned, how it is oriented, and in what direction it is moving. Further, all these things require a reference frame: a world-anchored space-defining framework with respect to which position, orientation, and movement can be specified. There also needs to be some kind of metric coordinate system - that is, a signal that specifies distances and directions with respect to this reference frame. The core question for animal navigation,

Kathryn (Kate) Jeffery is Professor of Behavioural Neuroscience at University College London (UCL), United Kingdom. After graduating in 1985 in medicine from the University of Otago, New Zealand, she embarked on a research career to study neural representation, training with Cliff Abraham (University of Otago), Richard Morris (University of Edinburgh), and John O'Keefe (UCL). In 1999 she joined the Psychology Department (now Division of Psychology \& Language Sciences) at UCL, where she continues research into the neural basis of spatial cognition.

Aleksandar Jovalekic currently is a Postdoctoral Fellow at the Institute of Neuroinformatics in Zurich. He obtained his PhD at University College London in 2012, where his doctoral work focused on how rodents navigate in three-dimensional environments.

Madeleine Verriotis is a Postdoctoral Research Associate at University College London (UCL). She received her BSc in Psychology and her PhD in Behavioural Neuroscience from UCL, during which she explored the representation of three-dimensional space in the rat and human brains. Her postdoctoral research focuses on the mechanisms underlying the development of pain processing in the human infant brain.

Robin Hayman is a Postdoctoral Research Associate in Neuroscience at University College London (UCL). He gained his BSc in Psychology with Literature at the University of Staffordshire, MSc in Neuroscience at UCL, and PhD in Psychology also at UCL. therefore, concerns what the reference frame might be and how the coordinate system encodes distances and directions within the frame.

The majority of studies of navigation, at least within neurobiology, have up until now taken place in laboratory settings, using restricted environments such as mazes, which are characterized both by being planar (i.e., two-dimensional) and by being horizontal. The real world differs in both of these respects. First, it is often not planar-air and water, for example, allow movement in any direction and so are volumetric. In this article, therefore, we will divide three-dimensional environments into those that are locally planar surfaces, which allow movement only in a direction tangential to the surface, and those that are volumetric spaces (air, water, space, and virtual reality), in which movement (or virtual movement) in any direction is unconstrained. Second, even if real environments might be (locally) planar, the planes may not necessarily be horizontal. These factors are important in thinking about how subjects encode and navigate through complex spaces.

What does the vertical dimension add to the problem of navigation? At first glance, not much: The vertical dimension provides a set of directions one can move in, like any other dimension, and it could be that the principles that have been elucidated for navigation in two dimensions extend straightforwardly to three. In fact, however, the vertical dimension makes the problem of navigation considerably more complex, for a number of reasons. First, the space to be represented is much larger, since a volume is larger than a plane by a power of $3 / 2$. Second, the directional information to be encoded is more complex, because there are three planes of orientation instead of just one. Furthermore, rotations in orthogonal planes interact such that sequences of rotations have different outcomes depending on the order in which they are executed (i.e., they are non-commutative), which adds to processing complexity. Third, the vertical dimension is characterized by gravity, and by consequences of gravity such as hydrostatic or atmospheric pressure, which add both information and effort to the computations involved. And finally, there are navigationally relevant cues available for the horizontal plane that are absent in the vertical: for example, the position of the sun and stars, or the geomagnetic field.

The internal spatial representation that the brain constructs for use in large-scale navigation is often referred to as a "cognitive map" (O'Keefe \& Nadel 1978). The cognitive map is a supramodal representation that arises from the collective activity of neurons in a network of brain areas that process incoming sensory information and abstract higher-order spatial characteristics such as distance and direction. In thinking about whether cognitive maps are three-dimensional, it is useful to consider the various 3D mapping schemes that are possible.

Figure 1 shows three different hypothetical ways of mapping spaces, which differ in the degree to which they incorporate a representation of the vertical dimension. In Figure la, the metric fabric of the map-the gridfollows the topography but does not itself contain any topographical information: the map is locally flat. Such a surface is called, in mathematics, a manifold, which is a space that is locally Euclidean - that is, for any point in the space, its immediate neighbours will be related to it by the usual rules of geometry, even if more-distant points are related in a non-Euclidean way. For example, the surface of a 


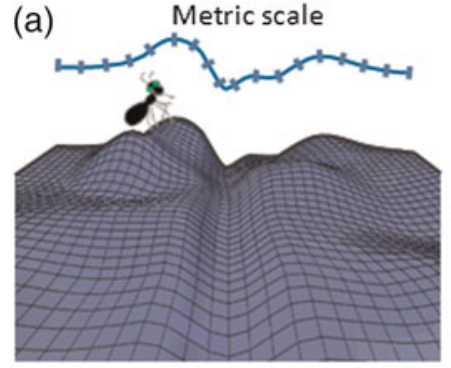

(b)

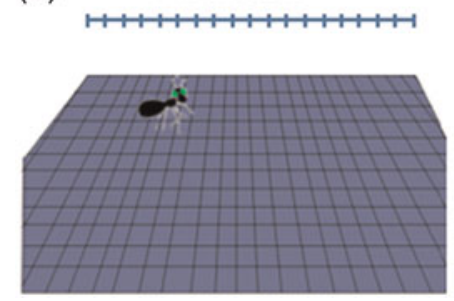

(c)
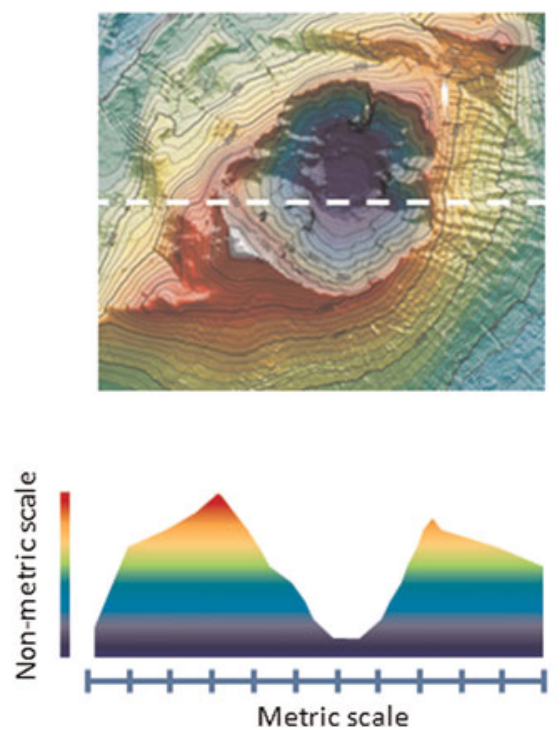

(d)

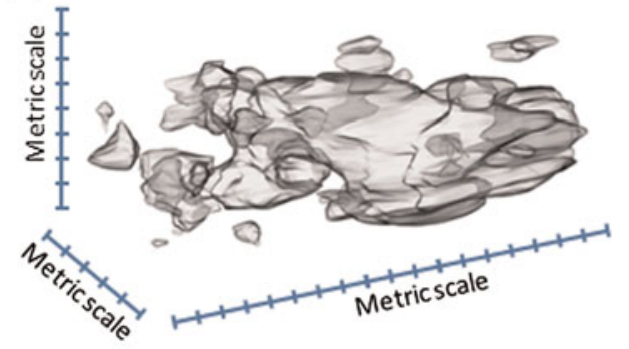

Figure 1. Different kinds of three-dimensional encoding. (a) A surface map, with no information about elevation. (b) An extracted flat map in which the horizontal coordinates have been generated, by trigonometric inference, from hilly terrain in (a). (c) A bicoded map, which is metric in the horizontal dimension and uses a non-metric scale (i.e., shading) in the vertical (upper panel = bird's eye view of the terrain, lower panel = cross-section at the level of the dotted line). (d) A volumetric map, which is metric in all three dimensions, like this map of dark matter in the universe.

sphere is a manifold, because a very small triangle on its surface will be Euclidean (its interior angles add up to 180 degrees) but a large triangle will be Riemannian (the sum of its interior angles exceeds 180 degrees). Given a small enough navigator and large enough surface undulations, the ground is therefore a manifold. For the purposes of the discussion that follows, we will call a map that follows the topography and is locally Euclidean a surface map. A surface map in its simplest form does not, for a given location, encode information about whether the location is on a hill or in a valley. ${ }^{2}$ Although such a map accurately represents the surface distance a navigator would need to travel over undulating terrain, because it does not contain information about height (or elevation) above a reference plane it would fail to allow calculation of the extra effort involved in moving over the undulations, and nor would the calculation of shortcuts be accurate.

A variant of the surface map is the extracted flat map shown in Figure 1b. Here, distances and directions are encoded relative to the horizontal plane, rather than to the terrain surface. For a flat environment, a surface map equates to an extracted flat map. For hilly terrain, in order to determine its position in $x-y$ coordinates, the navigator needs to process the slope to determine, trigonometrically, the equivalent horizontal distance. To generate such a map it is therefore necessary to have some processing of information from the vertical dimension (i.e., slope with respect to position and direction), and so this is a more complex process than for a purely surface map. There is evidence, discussed later, that some insects can in fact extract horizontal distance from hilly terrain, although whether this can be used to generate a map remains moot.

In Figure 1c, the map now does include a specific representation of elevation, but the encoding method is different from that of the horizontal distances. Hence, we have termed this a bicoded map. In a bicoded map, vertical distance is represented by some non-spatial variable - in this case, colour. Provided with information about $x-y$ coordinates, together with the non-spatial variable, navigators could work out where they were in three-dimensional space, but would not have metric information (i.e., quantitative information about distance) about the vertical component of this position unless they also had a key (some way of mapping the non-spatial variable to distances). Lacking a key, navigators could still know whether they were on a hill or in a valley, but could not calculate efficient shortcuts because they could not make the trigonometric calculations that included the vertical component. Such a map could be used for long-range navigation over undulating terrain, but determining the most energy-efficient route would be complicated because this would require chaining together segments having different gradients (and therefore energetic costs), as opposed to simply computing a shortest-distance route. Although the representational scheme described here is an artificial one used by human geographers, later on we will make the point that an analogous scheme-use of a non-metric variable to encode one of the dimensions in a three-dimensional map-may operate in the neural systems that support spatial representation.

Finally, the volumetric map shown in Figure $1 \mathrm{~d}$ could be used for navigation in a volumetric space. Here, all three dimensions use the same metric encoding scheme, and the shortest distance between any two points can be calculated straightforwardly using trigonometry (or some neural equivalent), with - on Earth - elevation being simply the distance in the dimension perpendicular to gravity. However, calculating energy costs over an undulating surface would still be complicated, for the same reasons as above-that is, energy expenditure would have to be continuously integrated over the course of the planned journey in order to allow comparisons of alternate routes. 
The questions concerning the brain's map of space, then, are: What encoding scheme does it use for navigation either over undulating surfaces or in volumetric spaces? Is the vertical dimension encoded in the same way as the two horizontal ones, as in the volumetric map? Is it ignored entirely, as in a surface map, or is it encoded, but in a different way from the horizontal dimensions, as in the bicoded map? These questions motivate the following analysis of experimental data and will be returned to in the final section, where we will suggest that a bicoded map is the most likely encoding scheme, at least in mammals, and perhaps even including those that can swim or fly.

\section{Behavioural studies in three dimensions}

A key question to be answered concerning the representation of three-dimensional space is how information from the vertical dimension is incorporated into the spatial representation. We will therefore now review experimental studies that have explored the use of information from the vertical dimension in humans and other animals, beginning with an examination of how cues from the vertical dimension may be processed, before moving on to how these cues may be used in self-localization and navigation. The discussion is organized as follows, based on a logical progression through complexity of the representation of the third (usually vertical) dimension:

Processing of verticality cues

Navigation on a sloping surface

Navigation in multilayer environments

Navigation in a volumetric space

\subsection{Processing of verticality cues}

Processing of cues arising from the vertical dimension is a faculty possessed by members of most phyla, and it takes many forms. One of the simplest is detection of the vertical axis itself, using a variety of mechanisms that mostly rely on gravity, or on cues related to gravity, such as hydrostatic pressure or light gradients. This information provides a potential orienting cue which is useful not only in static situations but also for navigation in three-dimensional space. Additionally, within the vertical dimension itself gravity differentiates down from up and thus polarizes the vertical axis. Consequently, animals are always oriented relative to this axis, even though they can become disoriented relative to the horizontal axes. The importance of the gravity signal as an orienting cue is evident in reduced or zero-gravity environments, which appear to be highly disorienting for humans: Astronauts report difficulty in orienting themselves in three-dimensional space when floating in an unfamiliar orientation (Oman 2007).

The vertical axis, once determined, can then be used as a reference frame for the encoding of metric information in the domains of both direction and distance. The technical terms for dynamic rotations relative to the vertical axis are pitch, roll, and yaw, corresponding to rotations in the sagittal, coronal, and horizontal planes, respectively (Fig. 2A). Note that there is potential for confusion, as these rotational terms tend to be used in an egocentric (body-centered) frame of reference, in which the planes of rotation move with the subject. The static terms used to describe the resulting orientation (or attitude) of the subject are elevation, bank, and azimuth (Fig. 2B), which respectively describe: (1) the angle that the front-back (antero-posterior) axis of the subject makes with earth-horizontal (elevation); (2) the angle that the transverse axis of the subject makes with earth-horizontal (bank); and (3) the angle that the antero-posterior axis of the subject makes with some reference direction, usually geographical North (azimuth). Tilt is the angle between the dorsoventral cranial axis and the gravitational vertical.

The other metric category, in addition to direction, is distance, which in the vertical dimension is called height - or (more precisely) elevation, when it refers to distance from a reference plane (e.g., ground or water level) in an upward direction, and depth when it refers to distance in a downward direction. Determination of elevation/depth is a special case of the general problem of odometry (distance-measuring).

There is scant information about the extent to which animals encode information about elevation. A potential source of such information could be studies of aquatic animals, which are exposed to particularly salient cues from the vertical dimension due to the hydrostatic pressure gradient. Many fish are able to detect this gradient via their swim bladders, which are air-filled sacs that can be inflated or deflated to allow the fish to maintain neutral buoyancy. Holbrook and Burt de Perera (2011a) have argued that changes in vertical position would also, for a constant gas volume, change the pressure in the bladder and thus provide a potential signal for relative change in height. Whether fish can actually use this information for vertical self-localization remains to be determined, however.

The energy costs of moving vertically in water also add navigationally relevant information. These costs can vary across species (Webber et al. 2000); hence, one might expect that the vertical dimension would be more salient in some aquatic animals than in others. Thermal gradients, which are larger in the vertical dimension, could also provide extra information concerning depth, as well as imposing differential energetic costs in maintaining thermal homeostasis at different depths (Brill et al. 2012; Carey 1992).

Interestingly, although fish can process information about depth, Holbrook and Burt de Perera (2009) found that they appear to separate this from the processing of horizontal information. Whereas banded tetras learned the vertical and horizontal components of a trajectory equally quickly, the fish tended to use the two components independently, suggesting a separation either during learning, storage, or recall, or at the time of use of the information. When the two dimensions were in conflict, the fish preferred the vertical dimension (Fig. 3), possibly due to the hydrostatic pressure gradient.

Several behavioural studies support the use of elevation information in judging the vertical position of a goal. For instance, rufous hummingbirds can distinguish flowers on the basis of their relative heights above ground (Henderson et al. 2001; 2006), and stingless bees can communicate the elevation as well as the distance and direction to a food source (Nieh \& Roubik 1998; Nieh et al. 2003). Interestingly, honeybees do not appear to communicate elevation (Dacke \& Srinivasan 2007; Esch \& Burns 1995). Rats are also able to use elevation information in goal localization, 
(a)

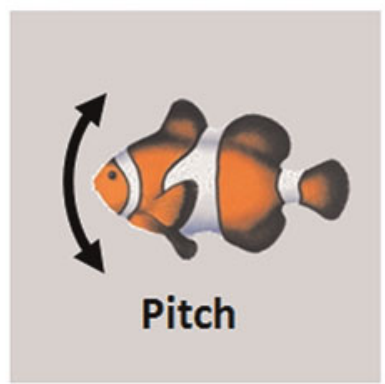

Sagittal plane
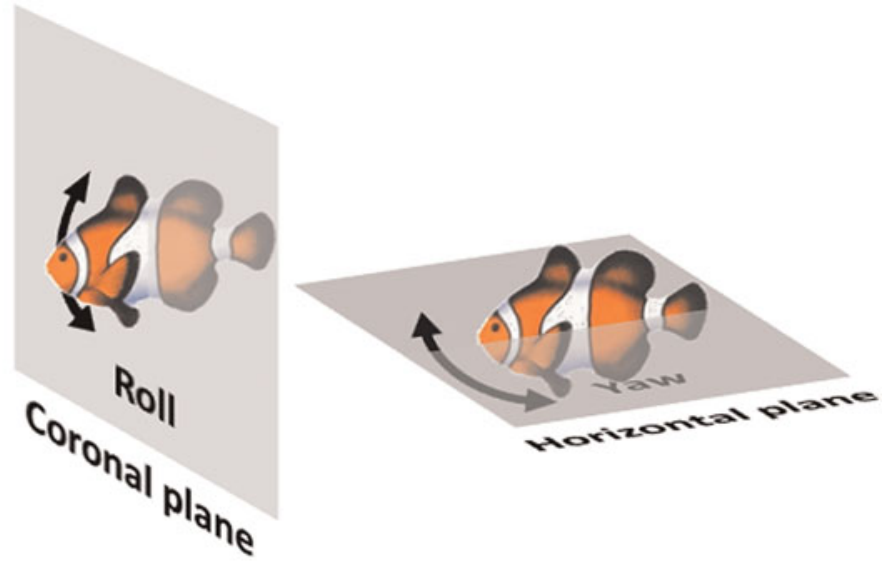

(b)
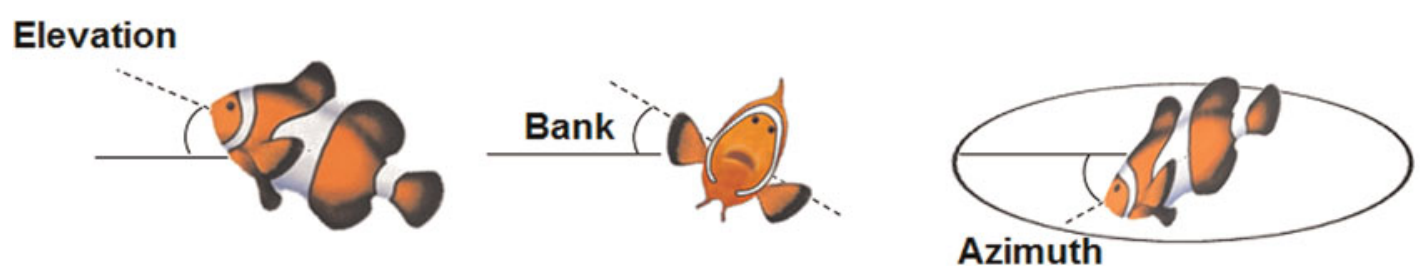

Figure 2. (a) Terminology describing rotations in the three cardinal planes. (b) Terminology describing static orientation (or "attitude") in each of the three planes.

and-like the fish in the Holbrook and Burt de Perera (2009) study - appear to separate horizontal and vertical components when they do so. For example, Grobéty \& Schenk (1992a) found that in a lattice maze (Fig. 4A), rats learned to locate the elevation of a food item more quickly than they learned its horizontal coordinates, suggesting that elevation may provide an additional, more salient spatial cue, perhaps because of its added energy cost, or because of arousal/anxiety.

Jovalekic et al. (2011) also found a vertical/horizontal processing separation in rats, but in the opposite direction from Grobéty and Schenk, suggesting a task dependence on how processing is undertaken. Rats were trained on a vertical climbing wall (studded with protruding pegs to provide footholds; Fig. 4B) to shuttle back and forth between a start and goal location. When a barrier was inserted into the maze, to force a detour, the rats overwhelmingly preferred, in both directions, the route in which the horizontal leg occurred first and the vertical leg second. Likewise, on a three-dimensional lattice similar to the one used by Grobéty and Schenk, the rats preferred the shallow-steep route to the steep-shallow route around a barrier. However, the task in Jovalekic et al.'s study differed from that faced by Grobéty and Schenk's rats in that in the latter, both the starting location and the goal were well learned by the time the obstacle was introduced. It may be that elevation potentiates initial learning, but that in a well-learned situation the animals prefer to solve the horizontal component first, perhaps due to an inclination to delay the more costly part of a journey.

Humans can estimate their own elevation, but with only a modest degree of accuracy. Garling et al. (1990) found that subjects were able to judge, from memory, which one of pairs of familiar landmarks in their hometown has a higher elevation, indicating that information about elevation is both observed and stored in the brain. It is not clear how this information was acquired, although there were suggestions that the process may be based partly on encoding heuristics, such as encoding the steepest and therefore most salient portions of routes first, as opposed to encoding precise metric information. Notwithstanding the salience of the vertical dimension, processing of the vertical dimension in humans appears poor (Montello \& Pick 1993; Pick \& Rieser 1982; Tlauka et al. 2007).

As with the fish and rodent studies above, a dissociation between vertical and horizontal processing was seen in a human study, in which landmarks arranged in a 3-by-3 grid within a building were grouped by most participants into (vertical) column representations, and by fewer participants into (horizontal) row representations (Büchner et al. 2007). Most participants' navigation strategies were consistent with their mental representations; as a result, when locating the given target landmark, a greater proportion of participants chose a horizontal-vertical route. The authors suggested that these results might have been reversed in a building that was taller than it was wide, unlike the building in their study. However, given the results from the rats of Jovalekic et al. (2011), in which the climbing wall dimensions were equal, it is also possible that this behaviour reflects an innate preference for horizontal-first routes.

In conclusion, then, studies of both human and nonhuman animals find that there is clear evidence of the use of elevation information in spatial computations, and also for differential processing of information from vertical versus horizontal dimensions. The processing differences between the two dimensions may result from differential experience, differential costs associated with the respective 


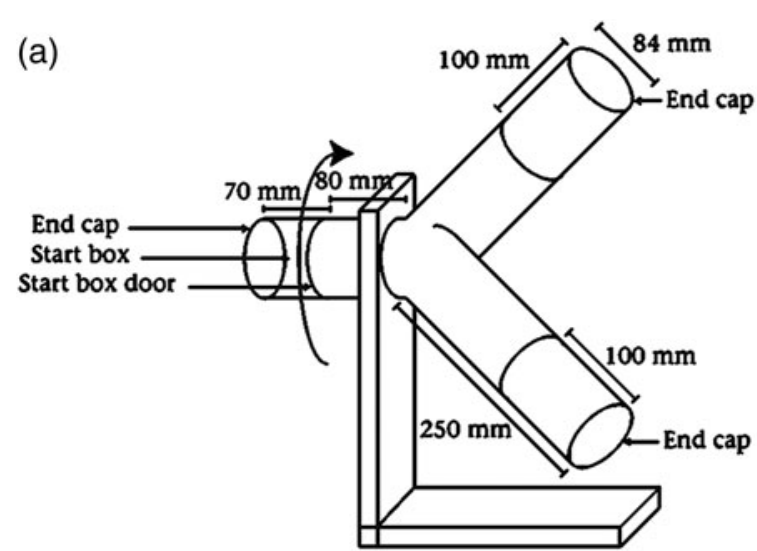

(b)

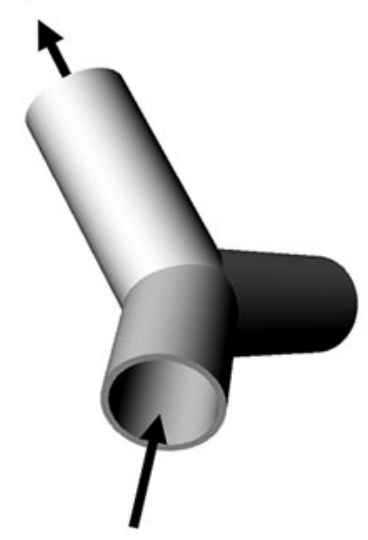

Training

(c)

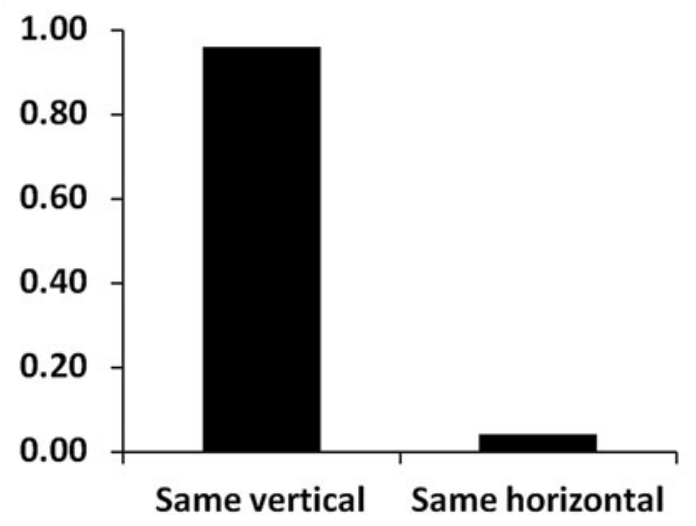

Figure 3. The experiment by Holbrook and Burt de Perera (2009). (a) Line diagram of the choice maze (taken from the original article, with permission), in which fish were released into a tunnel and had to choose one of the exit arms to obtain a reward. (b) The conflict experiment of that study, in which the fish were trained with the arms at an angle, so that one arm pointed up and to the left and the other arm pointed down and to the right. During testing, the arms were rotated about the axis of the entrance tunnel so that one arm had the same vertical orientation but a different left-right position, and one had the same horizontal (left-right) position but a different vertical level. (c) Analysis of the choices showed that the fish greatly preferred the arm having the same vertical level. Redrawn from the original. dimensions, or differences in how these dimensions are encoded neurally.

Next, we turn to the issue of how the change in height with respect to change in distance - that is, the slope of the terrain - may be used in spatial cognition and navigation. This is a more complex computation than simple elevation, but its value lies in the potential for adding energy and effort to the utility calculations of a journey.

\subsection{Navigation on a sloping surface}

Surfaces extending into the vertical dimension locally form either slopes or vertical surfaces, and globally form undulating or crevassed terrain. Slopes have the potential to provide several kinds of information to a navigating animal (Restat et al. 2004), acting, for instance, as a landmark (e.g., a hill visible from a distance) or as a local organizing feature of the environment providing directional information. Additionally, as described earlier, the added vertical component afforded by slopes is both salient and costly, as upward locomotion requires greater effort than horizontal locomotion, and an extremely steep slope could be hazardous to traverse. As a result, it is reasonable to assume that slopes might influence navigation in several ways.

A number of invertebrates, including desert ants and fiddler crabs, appear able to detect slopes, sometimes with a high degree of accuracy (at least 12 degrees for the desert ant; Wintergerst \& Ronacher 2012). It is still unknown how these animals perform their slope detection, which in the ant does not appear to rely on energy consumption (Schäfer \& Wehner 1993), sky polarization cues (Hess et al. 2009), or proprioceptive cues from joint angles (Seidl \& Wehner 2008). The degree to which slope is used in ant navigation has been under considerable investigation in recent years, making use of the ability of these animals to "home" (find their way back to the nest) using a process known as path integration (see Etienne \& Jeffery 2004; Walls \& Layne 2009; Wehner 2003) in which position is constantly updated during an excursion away from a starting point such as a nest.

Foraging ants will home after finding food, and the direction and distance of their return journey provide a convenient readout of the homing vector operative at the start of this journey. Wohlgemuth et al. (2001) investigated homing in desert ants and found that they could compensate for undulating terrain traversed on an outbound journey by homing, across flat terrain, the horizontally equivalent distance back to the nest, indicating that they had processed the undulations and could extract the corresponding ground distance. Likewise, fiddler crabs searched for their home burrows at the appropriate ground distance as opposed to the actual travelled distance (Walls \& Layne 2009). Whether such encoding of slope is relative to earthhorizontal (i.e., the plane defined by gravity) or whether it simply relates the two surfaces (the undulating outbound one and the flat return one), remains to be determined. However, despite the ability of Cataglyphis to extract ground distance, Grah et al. (2007) found that the ants did not appear to encode specific aspects of the undulating trajectory in their representations of the journey, as ants trained on outbound sloping surfaces will select homing routes that have slopes even when these are inappropriate (e.g., if the two outbound slopes had cancelled out, leaving 
(a)

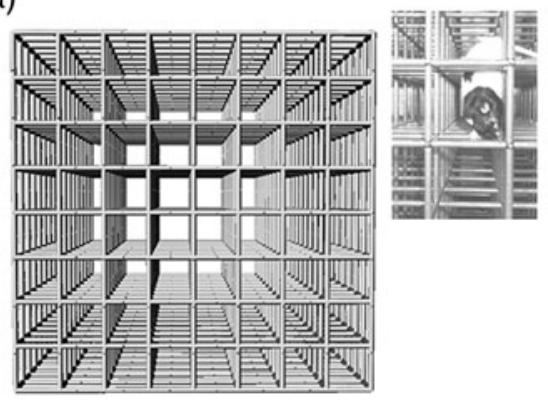

(b)

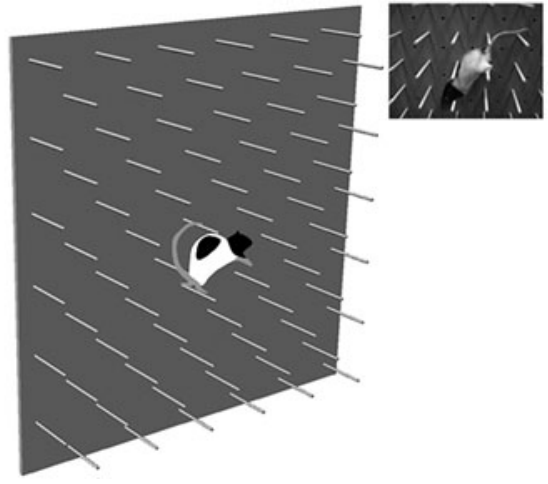

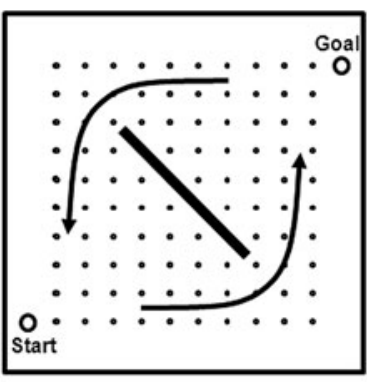

Figure 4. Rodent studies of vertical processing. (a) Schematic of the lattice maze of Grobéty and Schenk (1992a), together with a closeup photograph of a rat in the maze (inset). (b) Schematic and photograph of the pegboard maze used in the experiments of Jovalekic et al. (2011) and, as described later, by Hayman et al. (2011). In the Jovalekic et al. experiment, rats learned to shuttle directly back and forth between start and goal locations, as indicated in the diagram on the right. On test trials, a barrier (black bar) was inserted across the usual route, forcing the rats to detour. The great majority of rats preferred the routes indicated by the arrows, which had the horizontal leg first and the vertical leg second.

the ant at the same elevation as the nest with no need to ascend or descend). Thus, ants do not appear to incorporate elevation into their route memory; their internal maps seem essentially flat.

The ability of humans to estimate the slope of surfaces has been the subject of considerable investigation. Gibson and Cornsweet (1952) noted that geographical slant, which is the angle a sloping surface makes with the horizontal plane, is to be distinguished from optical slant, which is the angle the surface makes with the gaze direction. This is important because a surface appears to an observer to possess a relatively constant slope regardless of viewing distance, even though the angle the slope makes with the eye changes (Fig. 5A). The observer must somehow be able to compensate for the varying gaze angle in order to maintain perceptual slope constancy, presumably by using information about the angle of the head and eyes with respect to the body. However, despite perceptual slope constancy, perceptual slope accuracy is highly impaired - people tend to greatly overestimate the steepness of hills (Kammann 1967), typically judging a 5-degree hill to be 20 degrees, and a 30degree hill to be almost 60 degrees (Proffitt et al. 1995).

This mis-estimation of slope has been linked to the wellestablished tendency of people to underestimate depth in the direction of gaze (Loomis et al. 1992): If subjects perceive distances along the line of sight to be foreshortened, then they would perceive the horizontal component of the slope to be less than it really is for a given vertical distance, and thus the gradient to be steeper than it is. However, this explanation fails to account for why subjects looking downhill also perceive the hill as too steep (Proffitt et al. 1995). Furthermore, if observers step back from the edge of a downward slope, there is a failure of slope constancy as well as accuracy: they see the slope as steeper than they do if they are positioned right at the edge ( $\mathrm{Li} \&$ Durgin 2009). Durgin and Li have suggested that all these perceptual distortions can collectively be explained by so-called scale expansion (Durgin \& Li 2011; Li \& Durgin 2010) the tendency to overestimate angles (both gaze declination and optical slant) in central vision by a factor of about 1.5 (Fig. 5B). While this expansion may be beneficial for processing efficiency, it raises questions about how such distortions may affect navigational accuracy.
Other, nonvisual distortions of slope perception may occur, as well. Proffitt and colleagues have suggested that there is an effect on slope perception of action-related factors, such as whether people are wearing a heavy backpack or are otherwise fatigued, or are elderly (Bhalla \& Proffitt 1999); whether the subject is at the top or bottom of the hill (Stefanucci et al. 2005); and their level of anxiety (Stefanucci et al. 2008) or mood (Riener et al. 2011) or even level of social support (Schnall et al. 2012). However, the interpretation of many of these observations has been challenged (Durgin et al. 2012).

Creem and Proffitt $(1998 ; 2001)$ have argued that visuomotor perceptions of slope are dissociable from explicit awareness. By this account, slope perception comprises two components: an accurate and stable visuomotor representation and an inaccurate and malleable conscious representation. This may be why subjects who appear to have misperceived slopes according to their verbal reports nevertheless tailor their actions to them appropriately. Durgin et al. (2011) have challenged this view, suggesting that experimental probes of visuomotor perception are themselves inaccurate. The question of whether there are two parallel systems for slope perception therefore remains open.

How does slope perception affect navigation? One mechanism appears to be by potentiating spatial learning. For example, tilting some of the arms in radial arm mazes improves performance in working memory tasks in rats (Brown \& Lesniak-Karpiak 1993; Grobéty \& Schenk 1992b; Figure 6a). Similarly, experiments in rodents involving conical hills emerging from a flat arena that rats navigated in darkness found that the presence of the slopes facilitated learning and accuracy during navigation (Moghaddam et al. 1996; Fig. 6B). In these experiments, steeper slopes enhanced navigation to a greater extent than did shallower slopes. Pigeons walking in a tilted arena were able to use the 20-degree slope to locate a goal corner; furthermore, they preferred to use slope information when slope and geometry cues were placed in conflict (Nardi \& Bingman 2009b; Nardi et al. 2010). In these examples, it is likely that slope provided compass-like directional information, as well as acting as a local landmark with which to distinguish between locations in an environment. 
Jeffery et al.: Navigating in a three-dimensional world

(a)
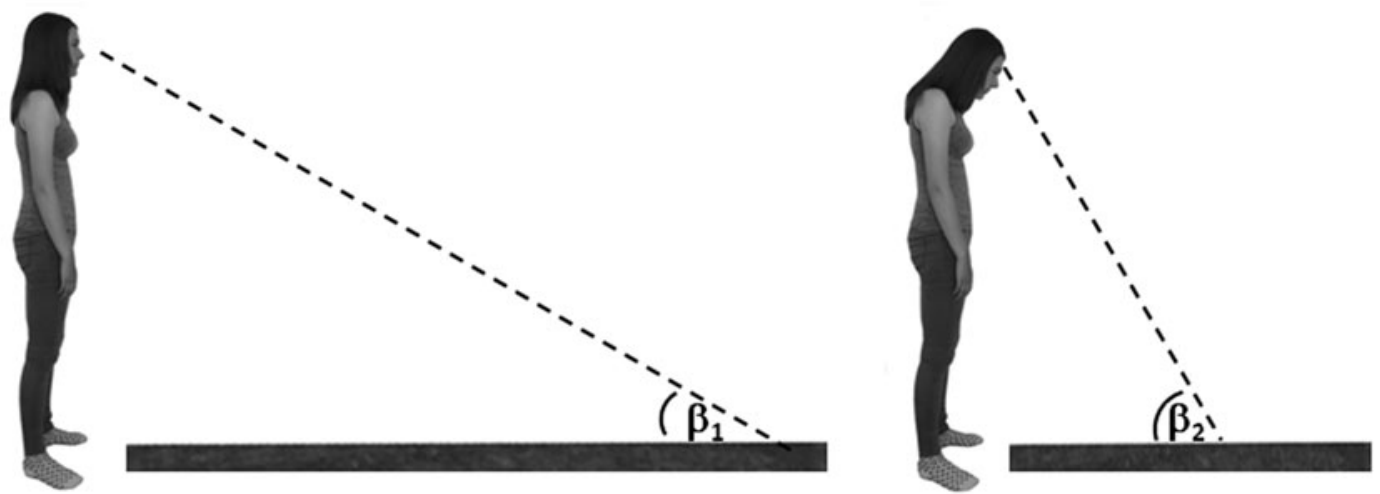

(b)

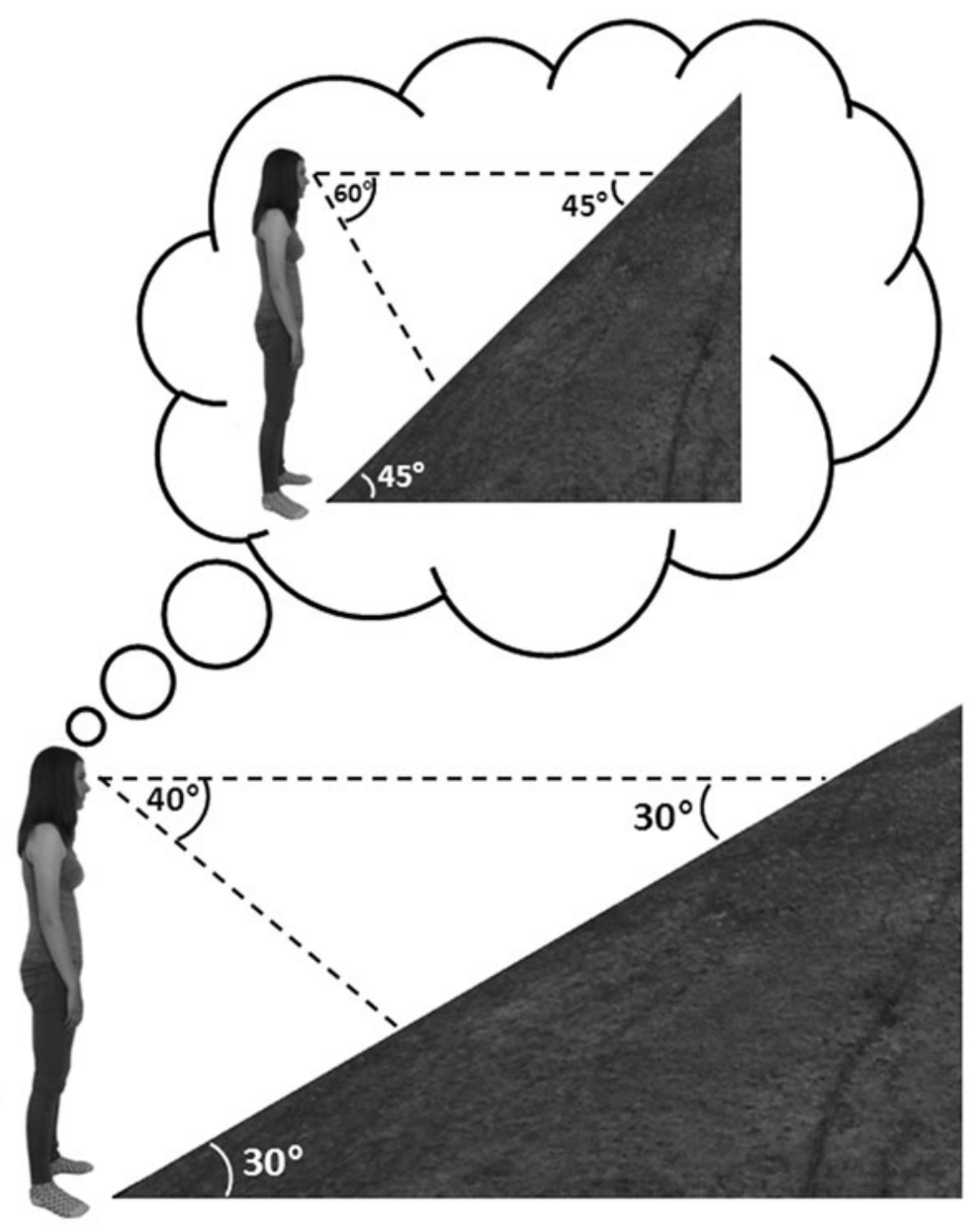

Figure 5. Visual slant estimation. (a) Slope constancy: A subject viewing a horizontal surface with a shallow gaze $\left(\beta_{1}\right)$ versus a steep gaze $\left(\beta_{2}\right)$ perceives the slope of the ground to be the same (i.e., zero) in both cases despite the optical slant ( $\beta$ ) being different in the two cases. In order to maintain constant slope perception, the nervous system must therefore have some mechanism of compensating for gaze angle. (b) The scale expansion hypothesis: An observer misperceives both the declination of gaze and changes in optical slant with a gain of 1.5, so that all angles are exaggerated but constancy of (exaggerated) slant is locally maintained. Increasing distance compression along the line of sight additionally contributes to slant exaggeration (Li \& Durgin 2010).

In humans, similar results were reported in a virtual reality task in which participants using a bicycle simulator navigated within a virtual town with eight locations (Restat et al. 2004; Steck et al. 2003; Fig. 6C). Participants experiencing the sloped version of the virtual town (4-degree slant) made fewer pointing and navigation errors than did those experiencing the flat version. This indicates that humans can also use slope to orient with greater accuracy -although, interestingly, this appears to be more true for males than for females (Nardi et al. 2011). When slope and geometric cues were placed in conflict, geometric information was evaluated as more relevant (Kelly 2011). This is the opposite of the finding reported in pigeons discussed above. There are, of course, a number of 
Jeffery et al.: Navigating in a three-dimensional world

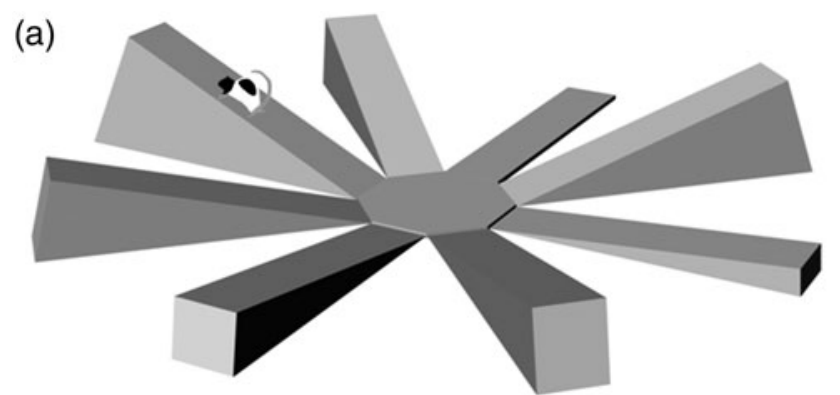

(b)

(c)

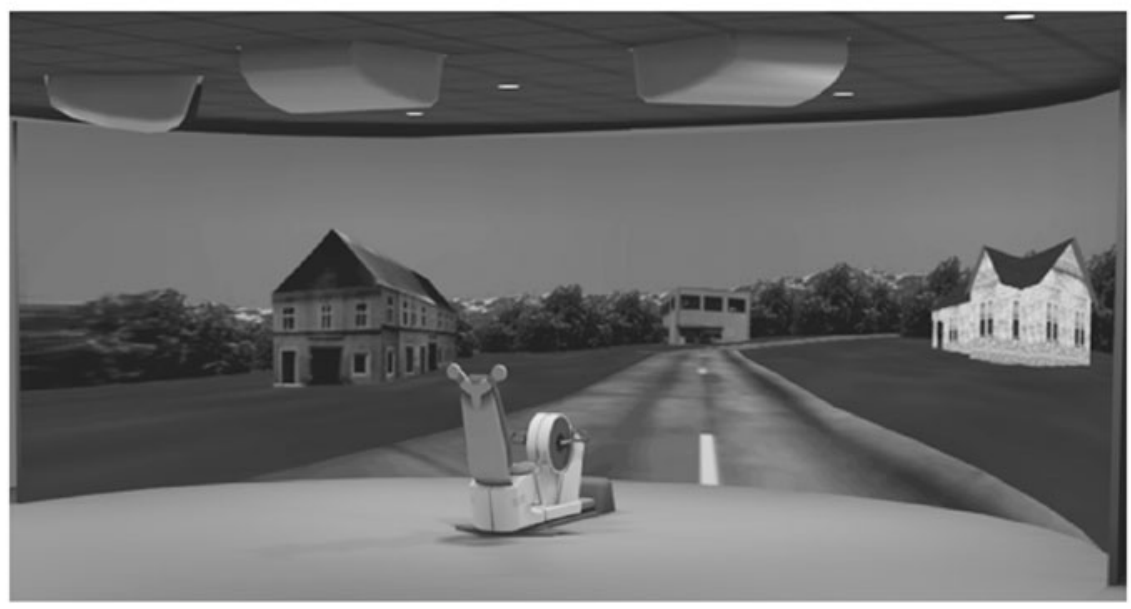

Figure 6. Behavioural studies of the use of slope cues in navigation, in rats (a and b) and in humans (c). (a) Schematic of the tilted-arm radial maze of Grobéty \& Schenk (1992b). (b) Schematic of the conical-hill arena of Moghaddam et al. (1996). (c) The virtual-reality sloping-town study of Restat et al. (2004) and Steck et al. (2003), taken from Steck et al. (2003) with permission.

reasons why pigeons and humans may differ in their navigational strategies, but one possibility is that different strategies are applied by animals that habitually travel through volumetric space rather than travel over a surface, in which geometric cues are arguably more salient.

The presence of sloping terrain adds complexity to the navigational calculations required in order to minimize energy expenditure. A study investigating the ability of human participants to find the shortest routes linking multiple destinations (the so-called travelling salesman problem, or "TSP") in natural settings that were either flat or undulating found that total distance travelled was shorter in the flat condition (Phillips \& Layton 2009). This was perhaps because in the undulating terrain, participants avoided straight-line trajectories in order to minimize hill climbing, and in so doing increased travel distance. However, it is not known to what extent they minimized energy expenditure. Interestingly, Grobéty \& Schenk (1992a) found that rats on the vertical plane made a far greater number of horizontal movements, partly because they made vertical translations by moving in a stair pattern rather than directly up or down, thus minimizing the added effort of direct vertical translations. This reflects the findings of the TSP in the undulating environments mentioned above (Phillips \& Layton 2009) and is in line with the reported horizontal preferences in rats (Jovalekic et al. 2011).

In summary, then, the presence of sloping terrain not only adds effort and distance to the animal's trajectory, but also adds orienting information. However, the extent to which slope is explicitly incorporated into the metric fabric of the cognitive map, as opposed to merely embellishing the map, is as yet unknown.

\subsection{Navigation in multilayer environments}

Multilayer environments - such as trees, burrow systems, or buildings - are those in which earth-horizontal $x-y$ coordinates recur as the animal explores, due to overlapping surfaces that are stacked along the vertical dimension. They are thus, conceptually, intermediate between planar and volumetric environments. The theoretical importance of multilayer environments lies in the potential for confusion, if - as we will argue later - the brain prefers to use a plane rather than a volume as its basic metric reference.

Most behavioural studies of spatial processing in multilayer environments have involved humans, and most human studies of three-dimensional spatial processing have involved multilayer environments (with the exception of studies in microgravity, discussed below). The core question concerning multilayer environments is the degree to which the layers are represented independently versus being treated as parts within an integrated whole. One way to explore this issue is to test whether subjects are able to path integrate across the lavers. Path integration, introduced earlier, consists of self-motion-tracking using visual, motor, and sensory-flow cues in order to continuously update representations of current location and/or a homing vector (for a review, see Etienne \& Jeffery 2004).

An early study of path integration in three dimensions explored whether mice could track their position across 
independent layers of an environment (Bardunias \& Jander 2000). The mice were trained to shuttle between their nestbox on one horizontal plane and a goal location on a parallel plane directly above the first, by climbing on a vertical wire mesh cylinder joining the two planes. When the entire apparatus was rotated by 90 degrees, in darkness while the animals were climbing the cylinder, all mice compensated for this rotation by changing direction on the upper plane, thus reaching the correct goal location. This suggests that they had perceived and used the angle of passive rotation, and mapped it onto the overhead space. A second control experiment confirmed that the mice were not using distal cues to accomplish this, thus suggesting that they were able to rely on internal path integration mechanisms in order to remain oriented in three dimensions.

However, it is not necessarily the case that the mice calculated a 3D vector to the goal, because in this environment the task of orienting in three dimensions could be simplified by maintaining a sense of direction in the horizontal dimension while navigating vertically. This is generally true of multilayer environments, and so to show true integration, it is necessary to show that subjects incorporate vertical angular information into their encoding. In this vein, Montello and Pick (1993) demonstrated that human subjects who learned two separate and overlapping routes in a multilevel building could subsequently integrate the relationship of these two routes, as evidenced by their ability to point reasonably accurately from a location on one route directly to a location on the other. However, it was also established that pointing between these vertically aligned spaces was less accurate and slower than were performances within a floor. Further, humans locating a goal in a multi-storey building preferred to solve the vertical component before the horizontal one, a strategy that led to shorter navigation routes and times (Hölscher et al. 2006). In essence, rats and humans appear in such tasks to be reducing a three-dimensional task to a one-dimensional (vertical) followed by a two-dimensional (horizontal) task. However, their ability to compute a direct shortest route was obviously constrained by the availability of connecting ports (stairways) between the floors, and so this was not a test of true integration.

Wilson et al. (2004) investigated the ability of humans to integrate across vertical levels in a virtual reality experiment in which subjects were required to learn the location of pairs of objects on three levels of a virtual multilevel building. Participants then indicated, by pointing, the direction of objects from various vantage points within the virtual environment. Distance judgements between floors were distorted, with relative downward errors in upward judgements and relative upward errors in downward judgements. This effect is interesting, as the sense of vertical space seems to be biased towards the horizontal dimension. However, in this study there was also a (slightly weaker) tendency to make rightward errors to objects to the left, and leftward errors to objects to the right. The results may therefore reflect a general tendency for making errors in the direction towards the center point of the spatial range, regardless of dimension (although this was more pronounced for the vertical dimension). Another interesting finding from this study is that there appears to be a vertical asymmetry in spatial memories, with a bias in favour of memories for locations that are on a lower rather than higher plane.
Tlauka et al. (2007) confirmed the findings of Wilson et al. (2004) and suggested that there might be a "contraction bias" due to uncertainty, reflecting a compressed vertical memory. They further speculated that such biases might be experience-dependent, as humans pay more attention to horizontal space directly ahead than to regions above or below it. This view is interesting and might explain the experimental results seen with rats in Jovalekic et al. (2011). Finally, young children appear to have more problems in such three-dimensional pointing tasks (Pick \& Rieser 1982), once again suggesting that experience may modulate performance in vertical tasks.

In the third part of this article, we will argue that the mammalian spatial representation may be fundamentally planar, with position in the vertical dimension (orthogonal to the plane of locomotion) encoded separately, and in a different (non-metric) way, from position in the horizontal (locomotor) plane. If spatial encoding is indeed planar, this has implications for the encoding of multilevel structures, in which the same horizontal coordinates recur at different vertical levels. On the one hand, this may cause confusion in the spatial mapping system: That is, if the representations of horizontal and vertical dimensions are not integrated, then the navigational calculations that use horizontal coordinates may confuse the levels. As suggested above, this may be why, for example, humans are confused by multilevel buildings. On the other hand, if there is, if not full integration, at least an interaction between the vertical and horizontal representations, then it is possible that separate horizontal codes are able to be formed for each vertical level, with a corresponding disambiguation of the levels. Further studies at both behavioural and neurobiological levels will be needed to determine how multiple overlapping levels are represented and used in navigation.

\subsection{Navigation in a volumetric space}

Volumetric spaces such as air and water allow free movement in all dimensions. Relatively little navigational research has been conducted in such environments so far. One reason is that it is difficult to track animal behaviour in volumetric spaces. The advent of long-range tracking methods that use global positioning system (GPS) techniques or ultrasonic tagging is beginning to enable research into foraging and homing behaviours over larger distances (Tsoar et al. 2011). However, many of these studies still address navigation only in the earth-horizontal plane, ignoring possible variations in flight or swim height. Laboratory studies have started to explore navigation in three-dimensional space, though the evidence is still limited.

One of the earliest studies of 3D navigation in rats, the Grobéty and Schenk (1992a) experiment mentioned earlier, involved a cubic lattice, which allowed the animals to move in any direction at any point within the cube (see Fig. 4A). As noted earlier, it was found that rats learned the correct vertical location before the horizontal location, suggesting that these elements may be processed separately. Grobéty and Schenk suggested that the rats initially paid greater attention to the vertical dimension both because it is salient and because greater effort is required to make vertical as opposed to horizontal translations; therefore, minimizing vertical error reduced their overall effort. 
Jeffery et al.: Navigating in a three-dimensional world

By contrast, Jovalekic et al. (2011) found that rats shuttling back and forth between diagonally opposite low and high points within a lattice maze or on a vertical subset of it, the pegboard (see Fig. 4B), did not tend to take the direct route (at least on the upward journey) but preferred to execute the horizontal component of the journey first. However, the separation of vertical from horizontal may have occurred because of the constraints on locomotion inherent in the maze structure: the rats may have found it easier to climb vertically than diagonally. Another experiment, however, also found vertical/horizontal differences in behavior: Jovalekic et al. (2011) examined foraging behaviour in the Grobéty and Schenk lattice maze and found that rats tended to retrieve as much of the food as possible on one horizontal layer before moving to the next. These authors observed similar behaviour on a simplified version of this apparatus, the pegboard, where again, foraging rats depleted one layer completely before moving to the next. The implication is that rather than using a truly volumetric representation of the layout, the animals tend to segment the environment into horizontal bands.

A similar propensity to treat three-dimensional environments as mainly horizontal has been reported in humans by Vidal et al. (2004), who studied participants in a virtual 3D maze and found that they performed better if they remained upright and had therefore aligned their egocentric and allocentric frames of reference in one of the dimensions. Similar results were also reported by Aoki et al. [2005]. Likewise, astronauts in a weightless environment tended to construct a vertical reference using visual rather than vestibular (i.e., gravitational) cues, and remain oriented relative to this reference (Lackner \& Graybiel 1983; Tafforin \& Campan 1994; Young et al. 1984).

Finally, one intriguing study has shown that humans may be able to process not only three- but also four-dimensional information (Aflalo \& Graziano 2008). In this virtual-reality study, in addition to the usual three spatial dimensions, a fourth dimension was specified by the "hot" and "cold" directions such that turns in the maze could be forward/ back, left/ right, up/down or hot/cold, and the usual trigonometric rules specified how "movements" in the fourth (hotcold) dimension related to movements in the other three. Participants were required to path integrate by completing a multi-segment trajectory through the maze and then pointing back to the start. They eventually (after extensive practice) reached a level of performance exceeding that which they could have achieved using three-dimensional reasoning alone. This study is interesting because it is highly unlikely that any animals, including humans, have evolved the capacity to form an integrated four-dimensional cognitive map, and so the subjects' performance suggests that it is possible to navigate reasonably well using a metrically lower-dimensional representation than the space itself. The question, then, is whether one can in fact navigate quite well in three dimensions using only a two-dimensional cognitive map. We return to this point in the next section.

\section{Neurobiological studies in three dimensions}

So far we have reviewed behavioural research exploring three-dimensional navigation and seen clear evidence that 3D information is used by animals and humans, although the exact nature of this use remains unclear. A parallel line of work has involved recordings from neurons that are involved in spatial representation. This approach has the advantage that it is possible to look at the sensory encoding directly and make fine-grained inferences about how spatial information is integrated. While most of this work has hitherto focused on flat, two-dimensional environments, studies of three-dimensional encoding are beginning to increase in number. Here, we shall first briefly describe the basis of the brain's spatial representation of two-dimensional space, and then consider, based on preliminary findings, how information from a third dimension is (or might be) integrated.

The core component of the mammalian place representation comprises the hippocampal place cells, first identified by O'Keefe and colleagues (O'Keefe \& Dostrovsky 1971), which fire in focal regions of the environment. In a typical small enclosure in a laboratory, each place cell has one or two, or sometimes several, unique locations in which it will tend to fire, producing patches of activity known as place fields (Fig. 7A). Place cells have therefore long been considered to represent some kind of marker for location. Several decades of work since place cells were first discovered have shown that the cells respond not to raw sensory inputs from the environment such as focal odours, or visual snapshots, but rather, to higherorder sensory stimuli that have been extracted from the lower-order sensory data-examples include landmarks, boundaries, and contextual cues such as the colour or odour of the environment (for a review, see Jeffery 2007; Moser et al. 2008).

For any agent (including a place cell) to determine its location, it needs to be provided with information about direction and distance in the environment. Directional information reaches place cells via the head direction system (Taube 2007; Taube et al. 1990a), which is a set of structures in areas surrounding the hippocampus whose neurons are selectively sensitive to the direction in which the animal's head is pointing (Fig. 7B). Head direction (HD) cells do not encode direction in absolute geocentric coordinates; rather, they seem to use local reference frames, determined by visual (or possibly also tactile) cues in the immediate surround. This is shown by the now well-established finding that rotation of a single polarizing landmark in an otherwise unpolarized environment (such as a circular arena) will cause the HD cells to rotate their firing directions by almost the same amount (Goodridge et al. 1998; Taube et al. 1990b). Interestingly, however, there is almost always a degree of under-rotation in response to a landmark rotation. This is thought to be due to the influence of the prevailing "internal" direction sense, sustained by processing of self-motion cues such as vestibular, proprioceptive, and motor signals to motion. The influence of these cues can be revealed by removing a single polarizing landmark altogether-the HD cells will maintain their previous firing directions for several minutes, although they will eventually drift (Goodridge et al. 1998).

The internal (sometimes called idiothetic) self-motion cues provide a means of stitching together the directional orientations of adjacent regions of an environment so that they are concordant. This was first shown by Taube and Burton (1995), and replicated by Dudchenko and Zinyuk (2005). However, although there is this tendency for the cells to adopt similar firing directions in adjacent 
Jeffery et al.: Navigating in a three-dimensional world

(a)

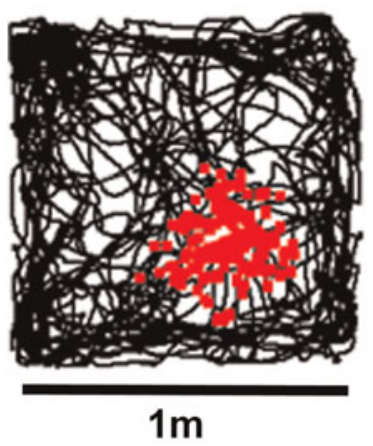

(b)

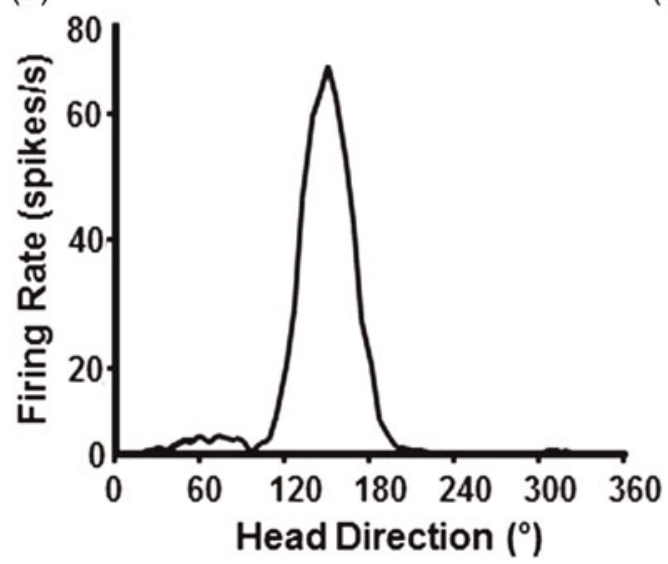

(c)

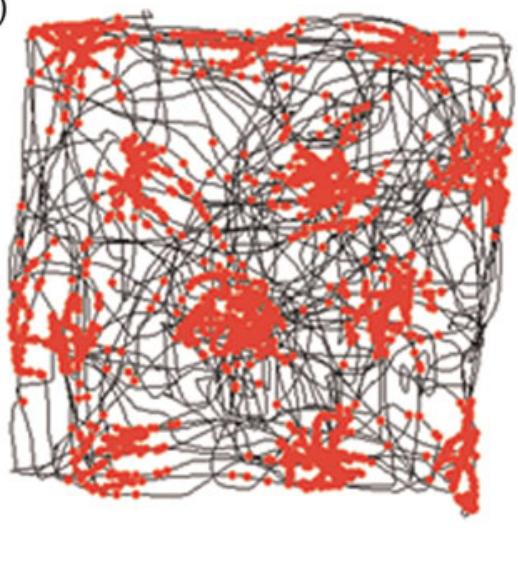

Figure 7. Firing patterns of the three principal spatial cell types. (a) Firing of a place cell, as seen from an overhead camera as a rat forages in a 1 meter square environment. The action potentials ("spikes") of the cell are shown as spots, and the cumulative path of the rat over the course of the trial is shown as a wavy line. Note that the spikes are clustered towards the South-East region of the box, forming a "place field" of about $40 \mathrm{~cm}$ across. (b) Firing of a head direction cell, recorded as a rat explored an environment. Here, the heading direction of the cell is shown on the $x$-axis and the firing rate on the $y$-axis. Note that the firing intensifies dramatically when the animal's head faces in a particular direction. The cell is mostly silent otherwise. (c) Firing of a grid cell (from Hafting et al. 2005), depicted as for the place cell in (a). Observe that the grid cell produces multiple firing fields in a regularly spaced array. Adapted from Jeffery and Burgess (2006).

environments, especially if the animal self-locomoted between them, this is not absolute and cells may have discordant directions, flipping from one direction to the other as the animal transitions from one environment to the next (Dudchenko \& Zinyuk 2005). This tendency for HD cells to treat complex environments as multiple local fragments is one that we will return to later.

The source of distance information to place cells is thought to lie, at least partly, in the grid cells, which are neurons in the neighbouring entorhinal cortex that produce multiple place fields that are evenly spaced in a grid-like array, spread across the surface of the environment (Hafting et al. 2005; for review, see Moser \& Moser 2008) (Fig. 7c). The pattern formed by the fields is of the type known as hexagonal close-packed, which is the most compact way of tiling a plane with circles.

Grid cell grids always maintain a constant orientation for a given environment (Hafting et al. 2005), suggesting a directional influence that probably comes from the headdirection system, as suggested by the finding that the same manipulations that cause HD firing directions to rotate also cause grids to rotate. Indeed, many grid cells are themselves also HD cells, producing their spikes only when the rat is facing in a particular direction (Sargolini et al. 2006). More interesting, however, is the influence of odometric (distance-related) cues on grid cells. Because the distance between each firing field and its immediate neighbours is constant for a given cell, the grid cell signal can theoretically act as a distance measure for place cells, and it is assumed that this is what they are for (Jeffery \& Burgess 2006) though this has yet to be proven. The source of distance information to the grid cells themselves remains unknown. It is likely that some of the signals are self-motion related, arising from the motor and sensory systems involved in commanding, executing, and then detecting movement through space. They may also be static landmark-related distance signals this is shown by the finding that subtly rescaling an environment can cause a partial rescaling of the grid array in the rescaled dimension (Barry et al. 2007), indicating environmental influences on the grid metric. It seems that when an animal enters a new environment, an arbitrary grid pattern is laid down, oriented by the (also arbitrary) HD signal. This pattern is then "attached" to that environment by learning processes, so that when the animal reenters the (now familiar) environment, the same HD cell orientation, and same grid orientation and location, can be reinstated.

Given these basic building blocks of the mammalian spatial representation, we turn now to the question of how these systems may cope with movement in the vertical domain, using the same categories as previously: slope, multilayer environments, and volumetric spaces.

\subsection{Neural processing of verticality cues}

The simplest form of three-dimensional processing is detection of the vertical axis. Verticality cues are those that signal the direction of gravity, or equivalently, the horizontal plane, which is defined by gravity. The brain can process both static and dynamic verticality cues: Static information comprises detection of the gravitational axis, or detection of slope of the terrain underfoot, while dynamic information relates to linear and angular movements through space and includes factors such as effort. Both static and dynamic processes depend largely on the vestibular apparatus in the inner ear (Angelaki \& Cullen 2008) and also on a second set of gravity detectors (graviceptors) located in the trunk (Mittelstaedt 1998). Detection of the vertical depends on integration of vestibular cues, together with those from the visual world and also from proprioceptive cues to head and body alignment (Angelaki et al. 1999; Merfeld et al. 1993; Merfeld \& Zupan 2002).

The vestibular apparatus is critical for the processing not only of gravity but also of movement-related inertial three- 
(a)

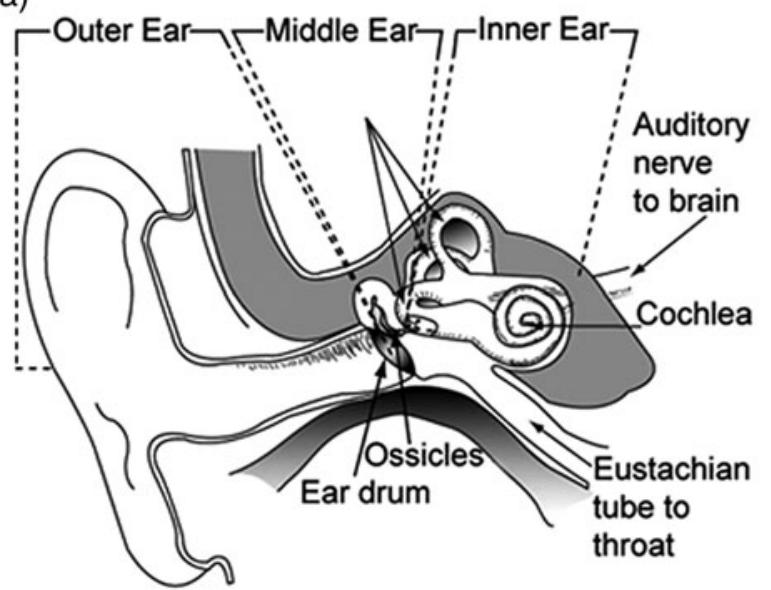

(b)

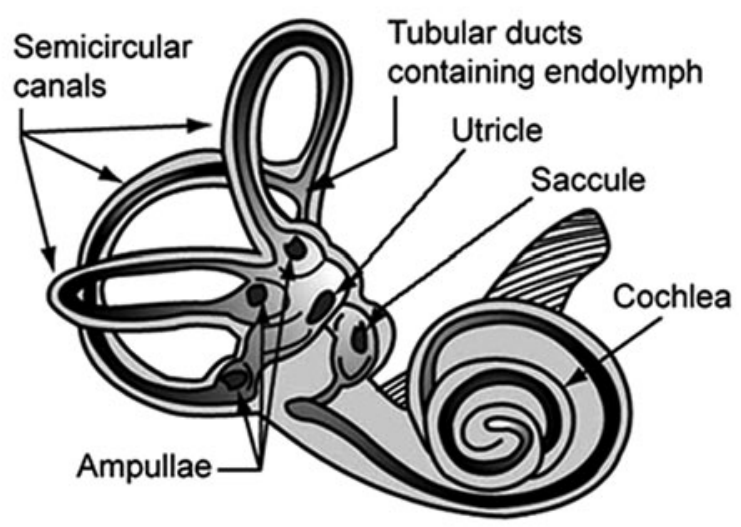

Figure 8. The vestibular apparatus in the human brain. (a) Diagram showing the relationship of the vestibular apparatus to the external ear and skull. (b) Close-up of the vestibular organ showing the detectors for linear acceleration (the otolith organs - comprising the utricle and saccule) and the detectors for angular acceleration (the semicircular canals, one in each plane). (Taken from: http://www.nasa.gov/ audience/forstudents/9-12/features/F_Human_Vestibular_System_in_Space.html) Source: NASA.

dimensional spatial cues. The relationship of the components of the vestibular apparatus is shown in Figure 8a, while the close-up in Figure 8b shows these core components: the otolith organs, of which there are two-the utricle and the saccule-and the semicircular canals. The otolith organs detect linear acceleration (including gravity, which is a form of acceleration) by means of specialized sensory epithelium. In the utricle the epithelial layer is oriented approximately horizontally and primarily detects earth-horizontal acceleration, and in the saccule it is oriented approximately vertically and primarily detects vertical acceleration/gravity. The two otolith organs work together to detect head tilt. However, because gravity is itself a vertical acceleration, there is an ambiguity concerning how much of the signal is generated by movement and how much by gravity - this "tilt-translation ambiguity" appears to be resolved by means of the semicircular canals (Angelaki \& Cullen 2008). These are three orthogonal fluid-filled canals oriented in the three cardinal planes, one horizontal and two vertical, which collectively can detect angular head movement in any rotational plane.

Neural encoding of direction in the vertical plane (tilt, pitch, roll, etc.) is not yet well understood. Head direction cells have been examined in rats locomoting on a vertical plane, and at various degrees of head pitch, to see whether the cells encode vertical as well as horizontal direction. Stackman and Taube (1998) found pitch-sensitive cells in the lateral mammillary nuclei (LMN). However, these were not true volumetric head direction cells, because their activity was not at all related to horizontal heading direction. Nor did they correspond to the vertical-plane equivalent of HD cells: Most of the observed cells had pitch preferences clustered around the almost-vertical, suggesting that these cells were doing something different from simply providing a vertical counterpart of the horizontal direction signal. By contrast, the HD cells in LMN were insensitive to pitch, and their firing was uniformly distributed in all directions around the horizontal plane. Thus, vertical and horizontal directions appear to be separately and differently represented in this structure.
In a more formal test of vertical directional encoding, Stackman et al. (2000) recorded HD cells as rats climbed moveable wire mesh ladders placed vertically on the sides of a cylinder. When the ladder was placed at an angular position corresponding to the cell's preferred firing direction, the cell continued to fire as the animal climbed the ladder, but did not fire as the animal climbed down again. Conversely, when the ladder was placed on the opposite side of the cylinder, the reverse occurred: now the cell remained silent as the animal climbed up, but started firing as the animal climbed down. This suggests that perhaps the cells were treating the plane of the wall in the same way that they usually treat the plane of the floor. Subsequently, Calton and Taube (2005) showed that HD cells fired on the walls of an enclosure in a manner concordant with the firing on the floor, and also informally observed that the firing rate seemed to decrease in the usual manner when the rat's head deviated from the vertical preferred firing direction. This observation was confirmed in a follow-up experiment in which rats navigated in a spiral trajectory on a surface that was either horizontal or else vertically aligned in each of the four cardinal orientations (Taube et al. 2013). In the vertical conditions, the cells showed preferred firing directions on the vertical surface in the way that they usually do on a horizontal surface. When the vertical spiral was rotated, the cells switched to a local reference frame and maintained their constant preferred firing directions with respect to the surface.

What are we to make of these findings? In the Calton and Taube (2005) experiment, it appears that the HD cells seemed to be acting as if the walls were an extension of the floor - in other words, as if the pitch transformation, when the rat transitioned from horizontal to vertical, had never happened. A possible conclusion is that HD cells are insensitive to pitch, which accords with the LMN findings of Stackman and Taube (1998). This has implications for how three-dimensional space might be encoded and the limitations thereof, which we return to in the final section. In the spiral experiment described by Taube (2005), it further appears that the reference frame provided 
by the locomotor surface became entirely disconnected from the room reference frame - when the rat was clinging to the vertical surface, the cells became insensitive to the rotations in the azimuthal plane that would normally modulate their firing on a horizontal plane. The idea of local reference frames is one that we will also come back to later.

The Calton and Taube (2005) experiment additionally replicated an observation made in microgravity, which is that head direction cells lose their directional specificity, or are greatly degraded, during inverted locomotion on the ceiling (Taube et al. 2004). In another study, head direction cells were monitored while animals were held by the experimenter and rotated by 360 degrees (relative to the horizontal plane) in the upright and in the inverted positions, as well as by 180 degrees in the vertical plane (Shinder \& Taube 2010). Although the animal was restrained, head direction cells displayed clear directionality in the upright and vertical positions, and no directionality in the inverted position. Additionally, directionality in the vertical position was apparent until the animal was almost completely inverted. These findings confirm those of the previous studies (Calton \& Taube 2005; Taube et al. 2004), that loss of HD cell directionality is a feature of the inverted position, at least in rats.

This breakdown in signalling may have arisen from an inability of the HD cells to reconcile visual evidence for the 180 degree heading-reversal with the absence of a 180 degree rotation in the plane to which they are sensitive (the yaw plane). This finding is consistent with a recent behavioural study by Valerio et al. (2010). Here, rats were trained to locomote while clinging upside-down to the underside of a circular arena, in order to find an escape hole. Animals were able to learn fixed routes to the hole but could not learn to navigate flexibly in a mapping-like way, suggesting that they had failed to form a spatial representation of the layout of the arena. Taking these findings together, then, it appears that the rodent cognitive mapping system is not able to function equally well at all possible orientations of the animal.

What about place and grid cells in vertical space? Processing of the vertical in these neurons has been explored in a recent study by Hayman et al. (2011). They used two environments: a vertical climbing wall (the pegboard), and a helix (Fig. 9). In both environments, place and grid cells produced vertically elongated fields, with grid fields being more elongated than place fields, thus appearing stripe-like (Fig. 9E). Hence, although elevation was encoded, its representation was not as fine-grained as the horizontal representation. Furthermore, the absence of periodic grid firing in the vertical dimension suggests that grid cell odometry was not operating in the same way as it does on the horizontal plane.

One possible explanation is that grid cells do encode the vertical axis, but at a coarser scale, such that the periodicity is not evident in these spatially restricted environments. If so, then the scale or accuracy of the cognitive map, at least in the rat, may be different in horizontal versus vertical dimensions, possibly reflecting the differential encoding requirements for animals that are essentially surface-dwelling. An alternative possibility, explored below, is that while horizontal space is encoded metrically, vertical space is perhaps encoded by a different mechanism, possibly even a non-grid-cell-dependent one. Note that because the rats remained horizontally oriented during climbing, it is also possible that it is not the horizontal plane so much as the current plane of locomotion that is represented metrically, while the dimension normal to this plane (the dorsoventral dimension with respect to the animal) is represented by some other means. Indeed, given the HD cell data discussed above, this alternative seems not only possible but likely. Thus, if the rats had been vertically oriented in these experiments, perhaps fields would have had a more typical, hexagonal pattern.

It is clear from the foregoing that much remains to be determined about vertical processing in the navigation system - if there is a vertical HD-cell compass system analogous to the one that has been characterized for the horizontal plane, then it has yet to be found and, if elevation is metrically encoded, the site of this encoding is also unknown. The cells that might have been expected to perform these functions - head direction, place, and grid cells - do not seem to treat elevation in the same way as they treat horizontal space, which argues against the likelihood that the mammalian brain, at least, constructs a truly integrated volumetric map.

We turn now to the question of what is known about the use of three-dimensional cues in navigationally relevant computations - processing of slopes, processing of multilayer environments, and processing of movement in volumetric space.

\subsection{Neural encoding of a sloping surface}

Investigation of neural encoding of non-horizontal surfaces is only in the early stages, but preliminary studies have been undertaken in both the presence and the absence of gravity. In normal gravity conditions, a slope is characterized by its steepness with respect to earth-horizontal, which provides important constraints on path integration. If the distance between a start location and a goal includes a hill, additional surface ground has to be travelled to achieve the same straight-line distance (i.e., that of flat ground). Furthermore, routes containing slopes require more energy to traverse than do routes without slopes, requiring a navigator to trade off distance against effort in undulating terrain. Therefore, one might imagine that slope should be incorporated into the cognitive map, and neural studies allow for an investigation of this issue that is not feasible with behavioural studies alone.

The effect of terrain slope has been explored for place cells but not yet for grid cells. The earliest place cell study, by Knierim and McNaughton (2001), investigated whether place fields would be modulated by the tilt of a rectangular track. A priori, one possible outcome of this experiment was that place fields would expand and contract as the tilted environment moved through intrinsically three-dimensional, ovoid place fields. However, fields that were preserved following the manipulation did not change their size, and many place cells altered their firing altogether on the track, evidently treating the whole tilted track as different from the flat track. One interpretation of these findings is that the track remained the predominant frame of reference (the "horizontal" from the perspective of the place cells), and that the tilt was signalled by the switching on and off of fields, rather than by parametric changes in place field morphology. 
(a)

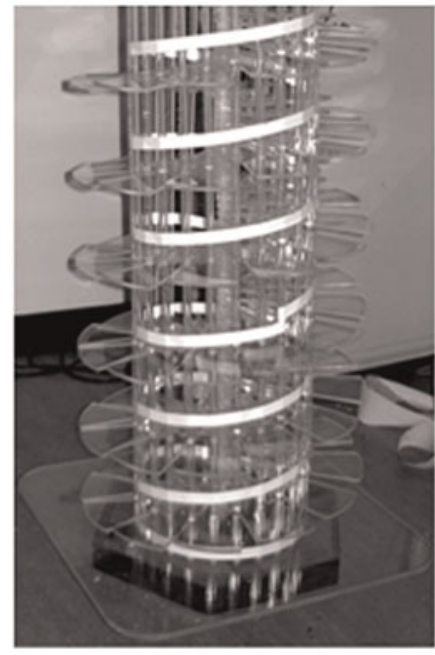

(b)

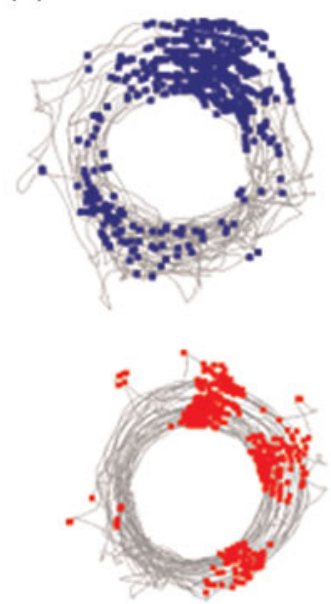

(c)

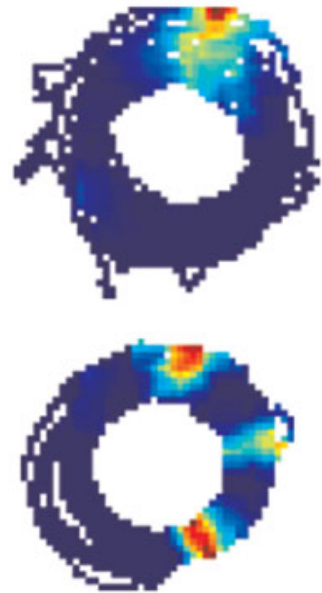

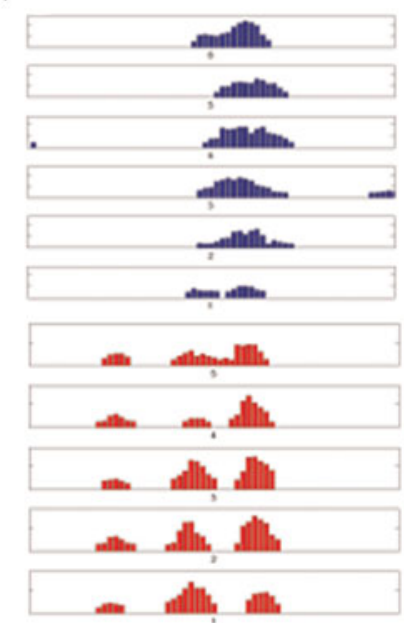

(d)

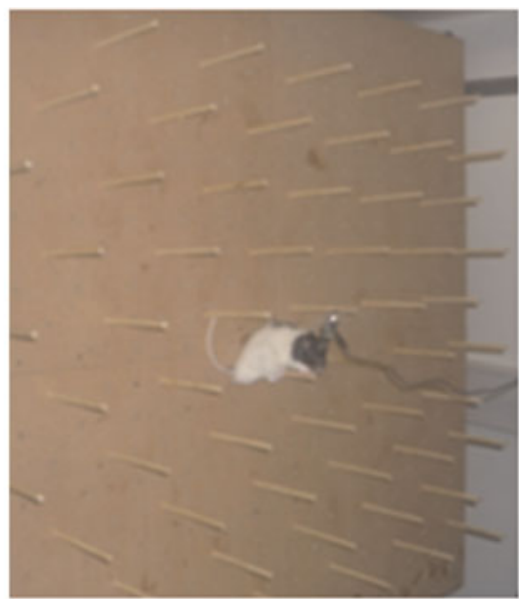

(e)
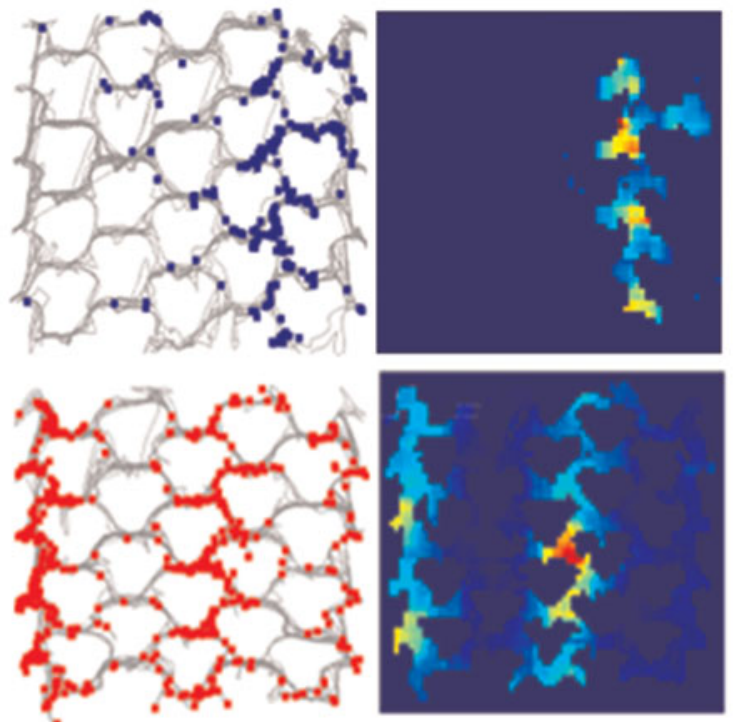

Figure 9. Adapted from Hayman et al. (2011). (a) Photograph of the helical maze. Rats climbed up and down either five or six coils of the maze, collecting food reward at the top and bottom, while either place cells or grid cells were recorded. (b) Left - The firing pattern of a place cell (top, spikes in blue) and a grid cell (bottom, spikes in red), as seen from the overhead camera. The path of the rat is shown in grey. The place cell has one main field with a few spikes in a second region, and the grid cell has three fields. Right - the same data shown as a heat plot, for clarity (red = maximum firing, blue = zero firing). (c) The same data as in (b) but shown as a firing rate histogram, as if viewed from the side with the coils unwound into a linear strip. The single place field in (b) can be seen here to repeat on all of the coils, as if the cell is not discriminating elevation, but only horizontal coordinates. The grid cell, similarly, repeats its firing fields on all the coils. (d) Photograph of the pegboard, studded with wooden pegs that allowed the rat to forage over a large vertical plane. (e) Left - The firing pattern of a place cell (top, spikes in blue) and a grid cell (bottom, spikes in red) as seen from a horizontally aimed camera facing the pegboard. The place cell produced a single firing field, but this was elongated in the vertical dimension. The grid cell produced vertically aligned stripes, quite different from the usual grid-like pattern seen in Fig. 8c. Right - the same data as a heat plot.

This study also showed that slope could be used as an orienting cue: In rotations of the tilted track, cells were more likely to be oriented by the track (as opposed to the plethora of distal cues) than in rotations of the flat track. This observation was replicated in a subsequent study by Jeffery et al. (2006), who found that place cells used the 30-degree slope of a square open arena as an orienting cue after rotations of the arena, and preferred the slope to local olfactory cues. Presumably, because the cells all reoriented together, the effect was mediated via an effect on the head direction system. Place cells have also been recorded from bats crawling on a near-vertical (70-degree tilt) open-field arena (Ulanovsky \& Moss 2007). Firing fields were similar to rodent place fields on a horizontal surface, a pattern that is consistent with the fields using the surface of the environment as their reference plane (because if earth-horizontal were the reference, then fields should have been elongated along the direction of the slope, as in the Hayman et al. [2011] experiment) (see Fig. 9e).

Grid cells have not yet been recorded on a slope. The pattern they produce will be informative, because their odometric properties will allow us to answer the question of whether the metric properties of place fields in horizontal environments arise from distances computed 
in earth-horizontal or with respect to environment surface. The results discussed above lead us to predict the latter.

Only one place cell study has been undertaken in the absence of gravity, in an intriguing experiment by Knierim and colleagues, conducted on the Neurolab Space Shuttle mission of 1998 (Knierim et al. 2000; 2003). Rats were implanted with microelectrodes for place cell recording, and familiarized with running on a rectangular track on Earth, before being carried in the shuttle into space. Under microgravity conditions in space, the animals were then allowed to run on a 3D track, the "Escher staircase," which bends through three-dimensional space such that three consecutive right-angle turns in the yaw plane of the animal, interspersed with three pitch rotations, leads the animal back to its starting point (Fig. 10a). The question was whether the cells could lay down stable place fields on the track, given that the visual cues might have conflicted with the path integration signal if the path integrator could not process the 3D turns. On the rats' first exposure to the environment on flight day 4, the cells showed somewhat inconsistent patterns, with those from one rat showing degraded spatial firing, those from a second rat showing a tendency to repeat their (also degraded) firing fields on each segment of the track, and those from a third looking like normal place fields. By the second recording session, on flight day 9, place fields from the first two rats had gained normal-looking properties, with stable place fields (Fig. 10b). This suggests either that the path integrator could adapt to the unusual conditions and integrate the yaw and pitch rotations, or else that the visual cues allowed the construction of multiple independent planar representations of each segment of the track.

It will be important to answer the question of which of these explanations is correct. If the cells could integrate turns in three dimensions, this would imply a highly sophisticated ability of the head direction system to co-ordinate turns in the three axes of rotation, which - given that they respond mainly to yaw-suggests an integrative process outside the HD cells themselves.

\subsection{Neural encoding of multilayer environments}

Very little work has examined neural encoding of space in multilayer environments, which is unfortunate given the theoretical importance of this issue, discussed earlier. Stackman et al. (2000) compared the firing of HD cells between the floor of a cylinder and an annular mezzanine ("annulus") located $74 \mathrm{~cm}$ above the floor, and found a $30 \%$ increase in firing rate on the annulus. However, the wire-mesh studies of Stackman et al. (2000) and Calton and Taube (2005) did not report increases in firing rate as animals climbed the walls, so the height effect may perhaps have been due to arousal or anxiety on the annulus. Directional firing preferences were shifted slightly on the upper level with respect to the lower, which may have resulted from transient slight disorientation during the climb.

There is also little work on place and grid cells in multilayer environments. Fenton et al. (2008) recorded place cells in a large environment, the walls of which supported stairs leading to an upper ledge housing reward drinking ports. They found that, frequently, fields that were present on the floor were also present on the corresponding part of the stairs/ledge. This could mean that either the fields were three-dimensional and extended from the floor to the staircase, or else that the cells were only responding to horizontal coordinates and ignoring the vertical. Results from the Hayman et al. (2011) experiment on the helical track support the latter interpretation, in that the data showed that firing tended to repeat on each successive coil (Fig. 9, b and c). There was modulation of firing rate across the coils, which is not inconsistent with the notion that the fields may have been intrinsically three-dimensional (albeit very elongated in the vertical dimension). However, it could also simply be rate modulation of the same field on each coil. Rate (a)

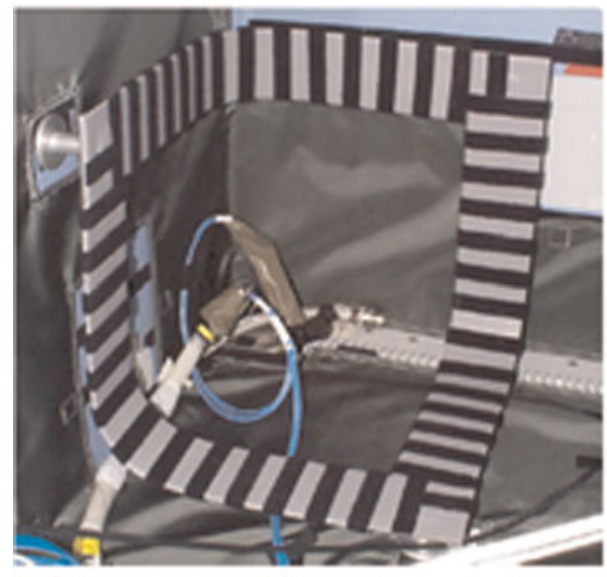

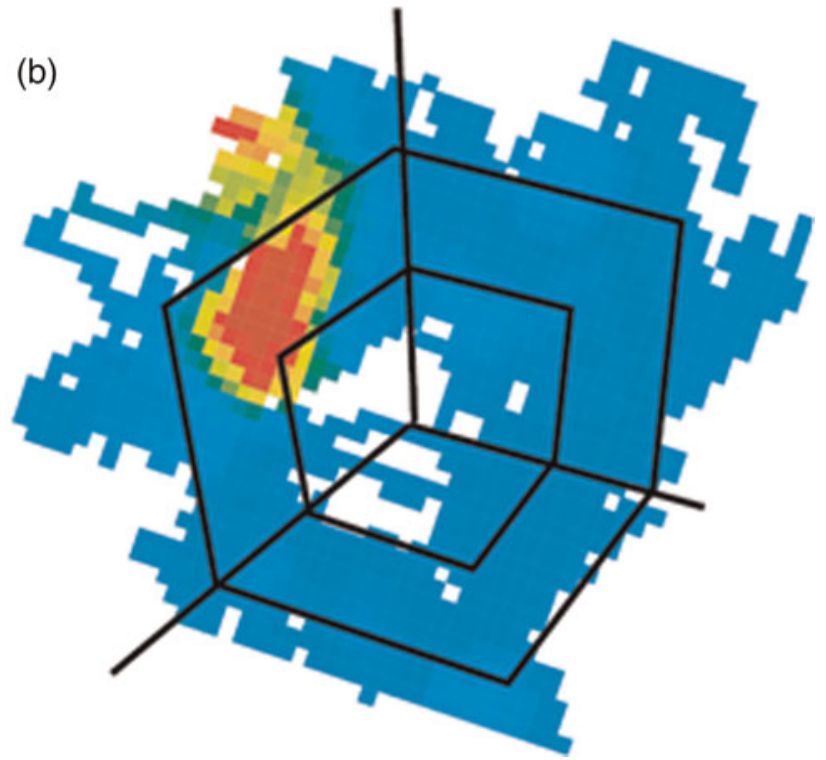

Figure 10. (a) The "Escher staircase" track, on which rats ran repeated laps while place fields were recorded. (b) The firing field of a place cell, showing a consistent position on the track, indicating either the use of visual cues or of three-dimensional path integration (or both) to position the field. (From Knierim et al. [2000] with permission.) 
modulation of place fields has been seen in other situations (Leutgeb et al. 2004) and suggests an influence of some other cues (in this case, elevation) on field production. Grid cells in the Hayman et al. experiment showed a similar pattern, with field location repeating from one coil to the next, but also (as with the place cells) a slight degree of rate modulation-something, interestingly, that has not been reported in other experiments on grid cells.

The implication of these findings is that the system was treating the environment as a stack of horizontal planes, rather than as an integrated three-dimensional space. However, the helical track experiment is not a true test of multilayered environment representation, because there was no discrete boundary between layers as there is in, say, a multi-storey building. Also, the stereotypical nature of the route the animals ran might have precluded the formation of independent maps for each layer. A truly multi-storey apparatus will be required to resolve this issue.

\subsection{Neural encoding of a volumetric space}

The final and most important question concerning threedimensional spatial encoding is whether there is metric encoding of volumetric space. As with multilayer environments, there have been no studies done to date on neural responses to free movement through true volumetric spaces, and so the answer to this question remains open. However, it is interesting to speculate about what kinds of evidence we should look for.
A volumetric map requires direction and distance to be calculated for both the horizontal and vertical dimensions. We have already seen that there is no evidence as yet for three-dimensional head direction cells, at least in rodents; so either there are three-dimensional HD cells in some other region of the brain, or else the cognitive map has a way of combining the planar signal from the classic HD areas together with pitch or elevation information, perhaps from the lateral mammillary nuclei.

What about distance in three dimensions? Because grid cells seem to provide the odometric representation for horizontal distance by virtue of their regularly spaced grid fields, we could predict that the periodicity observed in the horizontal dimension might be part of a three-dimensional, lattice-like arrangement of spherical fields. There are two ways of close-packing spheres in a volume, termed hexagonal and face-centered cubic, respectively (Fig. 11a and b). Since we already know that the grid array on the horizontal plane is hexagonal close-packed, if either of these volumetric arrangements were to pertain, then we would predict it should be the hexagonal form.

Although no recordings have yet been made of grids in a volumetric space, the experiment by Hayman et al. (2011) of grids recorded on a vertical plane might have been expected to reveal evidence of periodicity in the vertical dimension, akin to what one would find by transecting a close-packed volume (Fig. 11c). However, that experiment found that the grid fields formed stripes on the vertical surface, implying instead that the true underlying pattern of grids in a volumetric space might be in the form of (a)
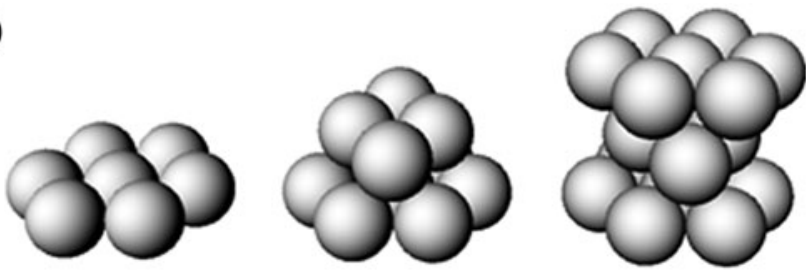

(b)
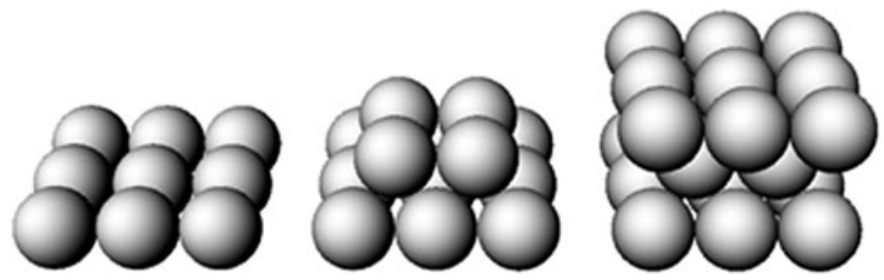

(c)

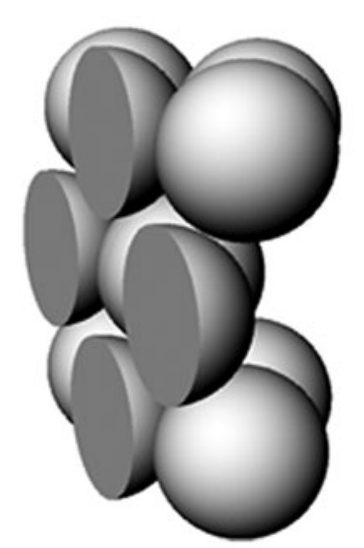

(d)

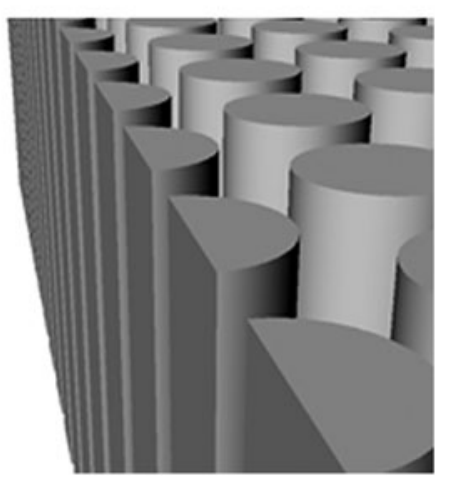

Figure 11. The two ways of efficiently filling a volumetric space with spheres: (a) Hexagonal close-packing, in which the spheres on each layer are planar hexagonal close-packed, and offset between one layer and the next and (b) Face-centered cubic, in which the spheres on each layer are in a square arrangement, with each sphere in a given layer nestling in the dip formed by four of those in the layers on either side. (c) The result of transecting a three-dimensional hexagonal close-packed array is a regular set of circles. (d) The result of transecting a set of horizontally hexagonal close-packed columns is stripes, resembling the grid cell pattern shown in Figure 9e. 
hexagonal close-packed columns (Fig. 11c). Whether this would also hold true for animals moving in an unconstrained way through the space remains to be seen. It is also possible that there is periodicity in the vertical dimension, but at a much greater scale than could be seen in a small laboratory setting.

\section{Is the cognitive map bicoded?}

At the beginning of this article we reviewed the encoding schemes that might exist to map three-dimensional space, highlighting three different mapping schemes that vary in their use of elevation information (see Fig. 1). Based on the foregoing review of behavioural and neurobiological studies, we return now to the question of map types. Below, we argue that the data collected to date favour a metrically planar map of the form we have termed "bicoded" - that is, using a different encoding scheme for horizontal than for vertical space. Indeed, we go a step further and suggest that the schemes are referenced not to horizontal and vertical, but, more generally, to the plane of locomotion (horizontal in the animal's canonical orientation) versus the axis orthogonal to this.

First, however, let us consider the alternative hypotheses for the possible structure of the cognitive map. These are (1) that the map is a surface map, lacking information about vertical travel, or (2) that it is a fully integrated volumetric map. It is unlikely that the cognitive map could be a surface map, because the evidence reviewed suggests that almost all animals investigated show some processing of vertical space. A volumetric map, by contrast, is metric (i.e., distances and directions are explicitly encoded) in all three dimensions. No studies have yet been undertaken in animals that can move freely in all three dimensions (such as those that fly or swim), and it may be that through evolution and/or through developmental experience, a fully integrated volumetric map may have formed in such animals. In particular, animals that have to solve three-dimensional spatial problems for which there is no planar approximation, such as those that have to path integrate across a volumetric space like the ocean, may be expected to possess a volumetric cognitive map if such a thing exists. Studies in birds, fish, or swimming and flying mammals such as cetaceans or bats have the potential to reveal whether there are spatial metric computations for travel in all three dimensions, and also whether these are integrated.

If the cognitive map in animals that have a truly threedimensional ecology is volumetric, we might expect to discover in them a new class of head direction cells that encode the vertical plane in the same way that the rodent HD cells discovered to date encode the horizontal plane. Alternatively, these animals might even possess HD cells that point uniquely to positions in three-dimensional space (i.e., that have tuning curves restricted in all three dimensions). We might also expect to see spherical grid cell grids in a 3D hexagonal close-packed array like those shown in Figure 1la and b.

\subsection{Arguments for a bicoded cognitive map}

The above possibilities concerning 3D maps notwithstanding, we suggest here that even ecologically three- dimensional animals may turn out to form planar, or quasi-planar, cognitive maps. Such animals often live in environments that are relatively more extended horizontally than vertically, making a planar map useful (particularly as almost all animals maintain a canonical body orientation with respect to gravity). Also, as discussed below, encoding three dimensions poses considerable technical challenges for a compass system. Indeed, there is little evidence for such integrated encoding in the species that have been studied so far. Head direction cells evidently operate in a plane rather than a volume, and grid cells are not periodic in the vertical dimension (or perhaps not in the dimension perpendicular to the animal's body plane).

In a bicoded map, there is a spatially metric representation of a reference plane-usually the horizontal plane - and a non-metric, or at least differently metricized, representation for the axis perpendicular to this plane. In the geographical example given earlier (Fig. 1c), the nonspatial indicator of distance was colour, which varies as a function of elevation but from which elevation cannot be extracted without a key. Loosely speaking, it is as if the non-metric variable conveys a coarse signal along the lines of "high" or "very high," but does not contain explicit information about distance that could enter into a trigonometric calculation.

Obviously, the cognitive map does not use colour as its non-metric variable, but it could plausibly use an analogous signal, which we call here, for want of a better word, "contextual" - that is, information that pertains to a space but need not itself contain distance or direction information. A real-world example of a contextual elevation cue might be hydrostatic pressure, which Burt de Perera and colleagues put forward as a possible mechanism for fish to localize themselves in the vertical axis (Burt de Perera et al. 2005). For terrestrial animals, it could be visual or kinaesthetic cues to ascent/descent, or aspects of the visual panorama; for flying animals, it could be depth cues from stereopsis or motion parallax, or perhaps olfactory gradients in the atmosphere.

Our hypothesis, then, is that the cognitive map is such a bicoded map - it is metric in a given plane (the plane of locomotion), and contextually modulated orthogonal to this. It is possible that contextual information concerning elevation could be processed by brain areas that are located outside the currently established navigation circuitry, although it is likely that these signals would interact at some point.

What evidence supports this bicoding view? Existing behavioural data from a number of studies, reviewed earlier, suggest a potential separation between horizontal and vertical dimensions. However, this does not prove that the encoding itself is different, merely that execution of navigational plans is different. Theoretically, this could be because the energetic costs of moving vertically are taken into account when planning, even if the planning is based on a fundamentally volumetric, three-dimensional map. The neural data, however, tell a more revealing story. The observation that head direction cells appear to use the plane of locomotion as a local reference (Stackman et al. 2000) implies that the directional signal for the vertical plane (or rather, the plane normal to locomotion) must lie elsewhere, in an as yet undiscovered region. Furthermore, the finding that grid and place cells have different metric properties in the vertical dimension, at least for horizontally 
oriented body posture, implies that odometry does not take place equally in all three dimensions - the map is anisotropic (Hayman et al. 2011).

Note that it remains to be determined experimentally whether there is something special about the horizontal plane in the cognitive map, or whether it is simply that gravity primarily orients the locomotor plane and it is this plane that is metrically encoded. Recordings of grid cells on steeply sloping surfaces may answer this question.

A bicoded scheme seems suboptimal, but in fact a surface-travelling animal could do quite well with such a map, because it could use the metrically encoded locomotor plane to calculate the directions needed for travel, and the topography of the landscape would constrain the animal to be at the correct elevation for that position. Although a bicoded map could not be used for path integration in volumetric space, an animal could divide the navigational task into two parts: It could first calculate the horizontal trajectory and then ascend to the correct elevation (as indicated by the contextual gradient), or vice versa-or both simultaneously but in separate computational circuits. Indeed, the findings of Grobéty and Schenk (1992a) seem to suggest that this is indeed what happens, at least in rats. However, in the case where "horizontal" is not true earth-horizontal, but lies on a slope, then it would be advantageous for the map to encode, in some form, information about that slope, as this would affect the energy costs of navigational routes involving that surface. Hence, there may be some mechanism for encoding the angle of a given planar fragment with respect to earth-horizontal.

A bicoded map has a planar metric reference, but as noted earlier, the real world is far from planar. How could a bicoded map be used on undulating topology? One possibility is that an undulating surface could be encoded as a manifold - not a single unified map, but a mosaic of contiguous map fragments. In such a scheme, each fragment would comprise a local reference frame defined by a reference plane (which would be horizontal on a horizontal surface, but need not be) and an axis orthogonal to this (which would correspond to the gravitationally defined vertical for an animal locomoting in its canonical orientation on a horizontal surface). Figure 12 shows an illustrative example of such a mosaicized representation. In the example, a rat, in a fairly short excursion, moves across the ground from a rock to a tree, up the trunk of the tree, and out onto a branch-each of these planes being orthogonal to the one before. To maintain orientation the animal must switch from a planar map referenced to the ground, to a new one referenced to the tree trunk, to a third referenced to the branch.

If the planar fragments of the cognitive map need not be oriented horizontally, then how does the animal process the vertical axis, which remains a special, and important, reference due to the effects of gravity? To form an integrated map of large-scale space using mosaic fragments, there also should be some means of associating the local reference frames, both to one another (to enable navigation between fragments) and to earth-horizontal (to enable appropriate energy and other gravity-related calculations to be made). A plausible means of achieving this could be the vestibular system, which tracks changes in orientation via the semi-circular canals and could therefore provide some means of linking the map fragments together at, so to speak, their "edges." With such a scheme it would be possible to use heuristic methods to compute, for example, approximate shortcuts between map fragments. For more sophisticated navigation, however, it would be preferable to weld fragments together into a larger, unitary frame that would allow for precise metric computations across the space. It may be that such a reference frame adaptation could occur with experience. In fact, the head direction cell studies of Stackman et al. (2000), in which reference frames

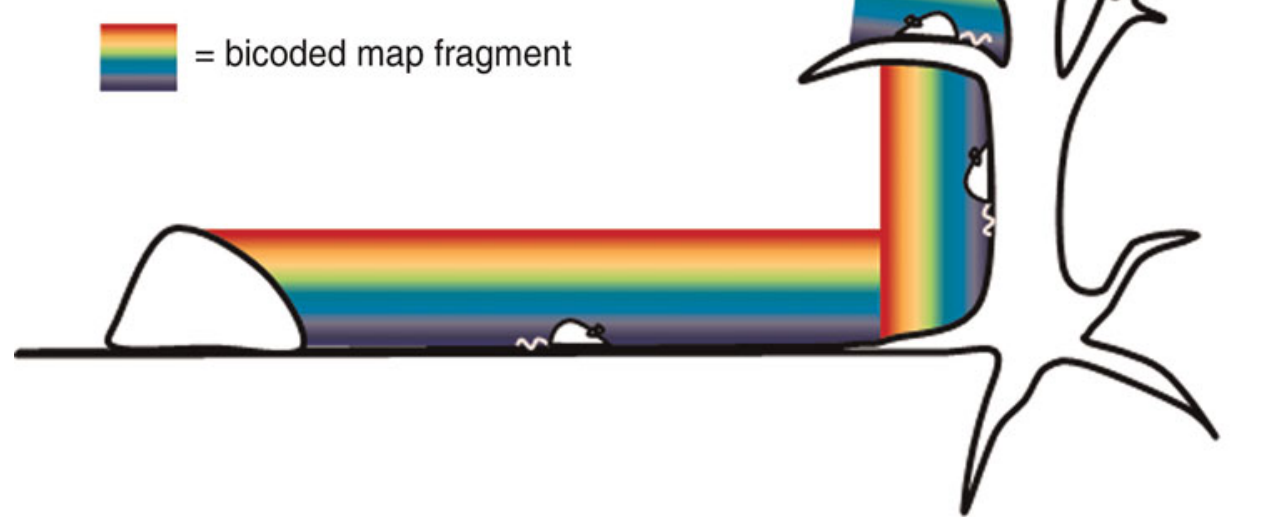

Figure 12. Hypothetical structure of the cognitive map in a dimensionally complex environment. The map is assumed to be metric in the plane of locomotion, which is the ground for the space between the rock and the tree, the vertical trunk as the animal climbs, and the horizontal branch. The shading gradient represents the nonmetric encoding, which conveys coarse information about distance in the plane orthogonal to the locomotor plane, which is encoded differently from the precise metric of the locomotor plane. 
could be local to the surface or more globally referenced to the surrounding room, support the possibility of such experience-dependent plasticity.

The possibility that the cognitive map is formed of a mosaic of locally planar fragments is consistent with the sudden reorientations experienced by humans and other animals as they transition from one region of the environment to the next (Wang \& Spelke 2002). It is a commonplace experience of urban navigators when taking an unfamiliar route between two locally familiar places, for example, that a sudden, almost dizzying re-alignment of reference frames occurs across the interface between the two regions. This subjective experience may reflect the sudden reorientation of head direction cells that occurs when animals cross between local connected regions having different head direction cell orientations (Dudchenko \& Zinyuk 2005; Taube \& Burton 1995; Zugaro et al. 2003). The reorientation episodes experienced by astronauts in conditions of microgravity may have a similar underlying cause (Oman 2007; See Fig. 12.)

What about subjects that habitually navigate in volumetric spaces, including animals such as fish, birds and bats, and also human divers, aviators and astronauts? Do they use a surface map too? In these environments, a plane-referenced map would seem much less useful. However, evidence from microgravity environment studies shows that even in a weightless environment, astronauts tend to find a reference plane to serve as a local "floor" (Oman 2007). This is usually the surface beneath their feet, but if they drift too far away from this "floor" and too far toward the "ceiling," or switch visual attention, they frequently report visual reorientation illusions (VRIs), where surfaces abruptly and unexpectedly exchange identity (Oman et al. 1986), testifying to the salience of the reference plane. Volume-travelling animals tend to maintain a constant body posture with respect to earthhorizontal and so they, too, may use a planar map for navigating, even though their movement in space is unconstrained.

\subsection{Why would animals have a metrically planar map?}

Why would animal cognitive maps be (quasi-)planar? On the face of it, this seems maladaptive, because a planar map, even a bicoded one, has inherently less information than a fully integrated volumetric one.

The first possibility is an ontogenetic one: that formation of the map during development is constrained by experience during infancy, and the animals that have been investigated to date have been, mainly for technical reasons, those species that are primarily surface-dwelling. An exception is fish, in which we also saw a processing separation between horizontal and vertical space (Holbrook \& Burt de Perera 2009), but this could have been a behavioural adaptation superimposed on an underlying volumetric map. It may be that if rats and mice were to be raised from birth in a volumetric space - for example, in microgravity, or at least in a lattice system in which they could move in all directions - then we would see true threedimensional HD cells, and path integration behaviour that seamlessly encompassed all three dimensions.

Second, it may be that phylogenetic development of the map was so constrained, and therefore, surface-dwelling animals such as rats, mice, and humans would never develop a 3D map even if raised in conditions that would allow it. In other words, surface-dwellers have either lost or else never evolved the neurological capacity to fully represent three-dimensional space. However, if we were to study HD cells in animals that can move freely in all dimensions, then we may find three-dimensional HD cells in these species. Emerging studies in bats may soon answer this question, we hope. It should be noted, however, that rats and mice naturally inhabit dimensionally complex environments, and therefore might be expected to show integrated three-dimensional encoding if this ever did evolve in vertebrates.

The third and final possibility - and one for which we argue here - is that a fully 3D map has never evolved in any species, because of the difficulties inherent in stably encoding an allocentric three-dimensional space using egocentric sensory receptors. A volumetric map requires three coordinates for position and three for direction. Monitoring position and travel in such a space is complicated because the vestibular inputs to the head direction system originate in the semicircular canals, which are themselves planar (one aligned in each plane). In order to extract threedimensional heading, the system would need to modulate the rotational signal from each canal with each of the other two, and do so dynamically and instantaneously. It is possible that the cost-benefit ratio of the required extra processing power is not sufficiently great, even for animals that have a three-dimensional ecological niche. As we saw in the four-dimension experiment of Aflalo and Graziano (2008), it is possible to navigate quite well in a space using a lower-dimensional representation, together with some heuristics, and what applies in four dimensions may equally well apply in three.

\section{Conclusion}

To conclude, then, we have reviewed emerging experimental evidence concerning the encoding of three-dimensional space in animals and humans. We find clear evidence of ability to process information about travel in the vertical dimension, particularly in the domain of slope intensity and slope direction. There is also some evidence of the processing of elevation information. Evidence for true volumetric coding is, however, weak, and both behavioural and neural studies suggest the alternative hypothesis that the neural representation of space, in a wide variety of species, is metrically flat (referenced to a plane-usually the plane of locomotion) and is modulated in the vertical dimension in a non-metric way. We have termed such a map "bicoded" to reflect its essentially anisotropic nature, and we suggest that a bicoded representation is a universal feature of vertebrate (and possibly invertebrate) cognitive maps.

\section{ACKNOWLEDGMENTS}

The work was supported by grants to Kate Jeffery from the Wellcome Trust (GR083540AIA), the European Commission Framework 7 ("Spacebrain”), the Medical Research Council (G1100669), and the Biotechnology and Biological Sciences Research Council (BB/J009792/1), and by a grant to Axona Ltd. from the European Commission Framework 7 ("Spacebrain").

\section{NOTES}

1. Madeleine Verriotis and Aleksandar Jovalekic contributed equally to this target article. 
2. Note that some other part of the animal's brain may be able to deduce, via postural and kinaesthetic mechanisms, the slope of the terrain. The point is that this information is not integrated into the metric fabric of the map itself.

\section{Open Peer Commentary}

\section{Semantic sides of three-dimensional space representation}

\author{
doi:10.1017/S0140525X13000307
}

\section{Arnaud Badets \\ Centre de Recherches sur la Cognition et l'Apprentissage, Centre National de la Recherche Scientifique (CNRS), UMR-7295, Maison des Sciences de l'Homme et de la Société, 86000 Poitiers, France. \\ arnaud.badets@univ-poitiers.fr \\ http://cerca.labo.univ-poitiers.fr}

\begin{abstract}
In this commentary, I propose that horizontal and vertical dimensions of space are represented together inside a common metrics mechanism located in the parietal cortex. Importantly, this network is also involved in the processing of number magnitudes and environmentdirected actions. Altogether, the evidence suggests that different magnitude dimensions could be intertwined with the horizontality and verticality of our world representation.
\end{abstract}

In their very attractive theory on navigation in a three-dimensional world, Jeffery et al. propose that our representations of the environment are based on cognitive maps, which separately integrate horizontal and vertical dimensions of space. However, knowledge of our environment is also built through our online motor behaviours, which improve the processing of other associated dimensions to flexibly adapt future responses. From this perspective, it has been suggested that a common metrics mechanism associated with sensorimotor experience is in charge of processing different magnitude dimensions, such as space, time, and numbers (Walsh 2003). My goal in this commentary is to suggest that a cognitive map based only on three-dimensional spaces might be an incomplete picture if abstract semantic dimensions are not fully considered. Accordingly, the representation of number magnitudes can also interact with the representation of the horizontal and vertical dimensions of space during performed actions and contribute to a global map of the three-dimensional world representation.

Evidence for a link among physical space, actions, and numbers comes from studies that show the association between small numbers with left and lower parts of space and large numbers with right and upper parts (Hubbard et al. 2005; Umiltà et al. 2009). For instance, Dehaene et al. (1993) found that small number processing can prime hand movement to the left part of space, and large numbers can prime movement to the right part. Schwarz and Keus (2004) revealed an association between the vertical dimension and eye movements - that is, downward and upward saccades were initiated more quickly in response to small and large numbers, respectively. Interestingly, Loetscher et al. (2010) found that during a random number generation task, the leftward and downward adjustment of eye locations predicted that the to-be-spoken number would be smaller than the last one. Conversely, a prediction of large numbers was made through the right and upward location of eyes. Altogether, these number-space associations occur in an automatic way, and magnitude representation could be represented as a number map that integrates spatial dimensions and actions (Schwarz \& Keus
2004; Walsh 2003). At a neurophysiological level, number magnitudes and three-dimensional Cartesian coordinates of external space are processed in a common parietal area (Hubbard et al. 2005). It is probable that daily life experience, such as adding more objects to a pile (for the vertical dimension), or cultural factors, such as the direction of writing (for the horizontal dimension), could partially be in charge of the link between numbers and spatially directed actions (Gevers et al. 2006). Another factor could also come from the fact that during infancy, counting strategies often involve finger movements, which in turn reinforce the number-space association through sensorimotor experience (Butterworth 1999; Michaux et al. 2010).

Based on sensorimotor accounts, Walsh's ATOM (“A Theory of Magnitude"; Walsh 2003) proposes that all magnitude dimensions (e.g., space, time, numbers, and lengths) are processed inside a common metrics mechanism located in the parietal cortex (Bueti $\&$ Walsh 2009). Note that neurons in the parietal cortex of different animal species, such as cats and macaque monkeys, can also respond to number processing (Nieder \& Miller 2004; Thompson et al. 1970). Importantly, the core assumption of ATOM is that we represent space and time through environment-directed actions. As stated by Walsh (2003), "the inferior parietal cortex reflects the common need for space, time and quantity information to be used in the sensorimotor transformations that are the main goal of these areas of cortex" (p. 483). It is worth noting that the parietal region is also strongly involved in route-based navigation in primates, especially in the integration of self-movement information (Sato et al. 2006). From an evolutionary viewpoint, one may wonder why such common metrics mechanisms exist. The most probable, straightforward answer is that the brains of human and nonhuman animals are mainly shaped for anticipating upcoming events in the environment (Corballis 2013; Hommel et al. 2001; Suddendorf \& Corballis 2007). On this view, we have recently revealed that the mere processing of number magnitudes is automatically associated with a general sensorimotor and anticipative mechanism (Badets et al. 2013). In this theory, distal references in the environment provide information of different magnitudes that is simulated in an anticipative way to flexibly adapt future numerical- and motor-based responses (see Badets \& Pesenti [2011] for this "anticipated-magnitude code").

Such anticipative mechanisms for sensorimotor adaptations are well documented in the literature on motor control (Hommel et al. 2001). For example, envisage a person who wishes to move a bottle of wine from the table to the upper part of the kitchen shelf. During this motor sequence, there is (1) the processing of a horizontal dimension for the reach-to-grasp movement of the hand towards the bottle on the table, and subsequently, (2) the processing of a vertical dimension for the placement of the bottle on the upper part of the shelf. According to Jeffery and colleagues, both dimensions (i.e., horizontal and vertical) should be processed and represented separately. However, data on similar paradigms revealed that when the final placement was high on the shelf (vertical goal), the reachto-grasp position of the hand (horizontal goal) was situated in the lower part of the object (Cohen \& Rosenbaum 2004). This finding indicates that the metric encoding the vertical goal is concomitantly anticipated and accurately represented during enactment of the horizontal goal.

In summary, based on several lines of evidence that a common metrics mechanism located in the parietal region of the brain is in charge of the processing of space, numbers, and actions, I propose that different magnitude dimensions are most likely intertwined with the horizontality and verticality of space during environment-directed actions. Semantic knowledge, such as the meaning of numbers, is represented inside this common scale and can refine the representation of physical space. In other words, semantic sides of three-dimensional space representation could be anticipatorily activated in a sensorimotor mechanism, which could give us the capacity to adapt different behaviours for potential environmental constraints. 


\section{Human path navigation in a three-dimensional world}

\section{doi:10.1017/S0140525X13000319}

\section{Michael Barnett-Cowan ${ }^{\mathrm{a}}$ and Heinrich H. Bülthoff ${ }^{\mathrm{b}, \mathrm{c}}$ \\ ${ }^{a}$ The Brain and Mind Institute, The University of Western Ontario, London, Ontario, N6A 5B7 Canada; ' ${ }^{b}$ Department of Human Perception, Cognition and Action, Max Planck Institute for Biological Cybernetics, 72076 Tübingen, Germany; ' Department of Brain and Cognitive Engineering, Korea University, Seoul, 136-713, Korea. \\ mbarnettcowan@gmail.com hhb@tuebingen.mpg.de \\ www.sites.google.com/site/mbarnettcowan/ www.kyb.mpg.de/ hhb}

\begin{abstract}
Jeffery et al. propose a non-uniform representation of threedimensional space during navigation. Fittingly, we recently revealed asymmetries between horizontal and vertical path integration in humans. We agree that representing navigation in more than two dimensions increases computational load and suggest that tendencies to maintain upright head posture may help constrain computational processing, while distorting neural representation of three-dimensional navigation.
\end{abstract}

The target article is a well-written and timely paper that summarizes much of the spatial cognition literature, which has only recently made headway into understanding how animals navigate in a threedimensional world. Most of what is known about how the brains of different animals manage navigation has been constrained to the horizontal plane. Here Jeffery et al. review experiments that show how insects, fish, rodents, and other animals (including humans) navigate space which includes a vertical component. From these studies, including some elegant work of their own on the encoding of three-dimensional space by place and grid cells (Hayman et al. 2011), the authors propose that 3D space is not uniformly represented by the brain. Rather, they suggest that 3D space is represented in a quasi-planar fashion, where spaces are constrained to separate planes that are stitched into a non-Euclidian, but integrated, map. There is, we think, merit in this analysis which presents a reasonable and testable hypothesis, one that addresses the need to reduce the computational load required to fully represent navigation through $3 \mathrm{D}$ space while also requiring a mechanism to stitch representational spaces together.

Given that the real world is three-dimensional, it is somewhat surprising that investigations into three-dimensional navigation have only recently emerged. One reason for this latency may be that representing movement in a volumetric space is not only computationally complicated for the brain, it poses additional constraints on experimental design, equipment, and analysis than are required for horizontal plane navigation. After reviewing the literature on nonhuman vertical navigation we became interested in human navigation with a vertical component. In a recent paper (Barnett-Cowan et al. 2012) we showed for the first time that human path integration operates differently in all three dimensions. We handled experimental constraints by comparing performance in an angle completion pointing task after passive translational motion in the horizontal, sagittal, and frontal (coronal) planes. To move participants in three dimensions, we took advantage of the unique Max Planck Institute (MPI) CyberMotion Simulator (Teufel et al. 2007), which is based on an anthropomorphic robot arm and which offers a large motion range to assess whether human path integration is similar between horizontal and vertical planes. We found that while humans tend to underestimate the angle through which they move in the horizontal plane (see also Loomis et al. 1993), either no bias or an overestimate of movement angle is found in the sagittal and frontal planes, respectively. Our results are generally in agreement with the theory proposed by Jeffery et al. for the representation of space being fixed to the plane of movement, and our approach lends itself well to testing predictions that follow from this theory. For example, if the representation of space is fixed to the plane of movement and additional processing is required to stitch planes together, then one would expect delays in response times to cognitive tasks as the number of planes moved through increases.
As Jeffery et al. point out, the constant force of gravity provides an allocentric reference for navigation. However, we would like to clarify that gravity provides an allocentric reference direction and not a reference frame. A reference direction is fundamental to perception and action, and gravity is ideally suited as a reference direction because it is universally available to all organisms on Earth for navigation and orientation. Knowing one's orientation and the orientation of surrounding objects in relation to gravity affects the ability to identify (Dyde et al. 2006), predict the behaviour of (Barnett-Cowan et al. 2011), and interact with surrounding objects (McIntyre et al. 2001), as well as to maintain postural stability (Kluzik et al. 2005; Wade \& Jones 1997). Tendencies to maintain upright head posture relative to gravity during self-motion and when interacting with objects have the functional advantage of constraining the computational processing that would otherwise be required to maintain a coherent representation of self and object orientation when the eyes, head, and body are differently orientated relative to one another and relative to an allocentric reference direction such as gravity. Such righting behaviour is expressed in Figure 1, where maintaining an upright head posture benefits gaze control for both fly and human.

In addition to studies of righting behaviour, there are now numerous studies which indicate that the brain constructs an internal representation of the body with a prior assumption that the head is upright (Barnett-Cowan et al. 2005; Dyde et al. 2006; MacNeilage et al. 2007; Mittelstaedt 1983; Schwabe \& Blanke 2008). We speculate that the combination of righting reflexes-to maintain an upright head posture during selfmotion and optimize object recognition-along with prior assumptions of the head being upright, may interfere with the brain's ability to represent three-dimensional navigation through volumetric space. Further, as vestibular and visual directional sensitivity are best with the head and body upright relative to gravity (MacNeilage et al. 2010), future experiments aimed at disentangling how the brain represents three-dimensional navigation must consider how the senses are tuned to work best when upright.

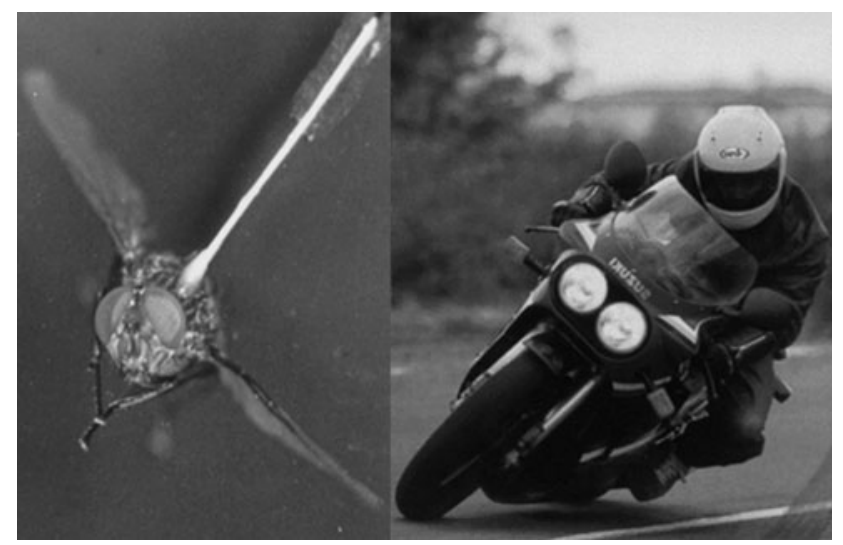

Figure 1 (Barnett-Cowan \& Bülthoff). Optimization of gaze control in the blowfly Calliphora (left; see Hengstenberg 1991) and in humans (right; see Brodsky et al. 2006), where upright head posture is maintained during self-motion. Republished from Hengstenberg (1991) with original photo copyright to MPI for Biological Cybernetics (left), and Bike Magazine (August 2001, p. 71; right), with permission."*

${ }^{*}$ Note: The photo shown on the left above was taken at the Max Planck Institute for Biological Cybernetics, which retains the copyright. Hengstenberg (1991) published part of this photograph as well. In addition to holding the copyright for the original photo we also have permission from the publisher of Hengstenberg's article. 
Understanding how humans represent motion through volumetric space is particularly important for assessing human aviation, where loss of control in flight is today's major single cause of fatal accidents in commercial aviation (see the EASA Annual Safety Review; European Aviation Safety Agency 2006). We suggest that our understanding of three-dimensional navigation in humans can be improved using advanced motion simulators - such as our Max Planck Institute CyberMotion Simulator, which is capable of moving human observers through complex motion paths in a volume of space. (An open access video is found in the original article: Barnett-Cowan et al. 2012.) Future experiments with fewer constraints, including trajectories using additional degrees of freedom, longer paths, multiple planes, and with the body differently orientated relative to gravity, will be able to more fully assess the quasi-planar representation of space proposed by Jeffery et al., as well as how vertical movement may be encoded differently.

\section{Learning landmarks and routes in multi- floored buildings}

\author{
doi:10.1017/S0140525X13000320
}

\section{Alain Berthoz ${ }^{\mathrm{a}}$ and Guillaume Thibault ${ }^{\mathrm{b}}$ \\ aLaboratoire de Physiologie de la Perception et de l'Action, Collège de France, 75231 Paris, France; 'blectricité de France Recherche \& Développement, 92141 Clamart cedex, France. \\ alain.berthoz@colllege-de-france.fr \\ guillaume.thibault@edf.fr \\ http://www.lppa.college-de-france.fr/EN/ \\ http://research.edf.com/research-and-innovation-44204.html}

\begin{abstract}
Existing studies have proposed that humans preferentially memorize buildings as a collection of floors. Yet this might stem from the fact that environments were also explored by floors. We have studied this potential bias with a learning and recognition experiment. We have detected a positive influence of the learning route-by floors, and also crucially by columns - on spatial memory performances.
\end{abstract}

This commentary addresses the target article's discussion of "navigation in multilayer environments" pace Jeffery et al.'s review of Montello and Pick's (1993) study, which "demonstrated that human subjects who learned two separate and overlapping routes in a multilevel building could subsequently integrate the relationship of these two routes. However, it was also established that pointing between these vertically aligned spaces was less accurate and slower than were performances within a floor" (sect. 3.3, para. 5). By questioning the underlying methodology we point out how horizontal learning and also vertical learning of multifloored environments influence spatial memory, which extends the findings reported by Jeffery et al.

Existing studies on spatial memory in complex buildings (Büchner et al. 2007; Hölscher et al. 2006; Montello \& Pick 1993) have suggested that humans have a tendency to memorize such environments as a collection of floors. Yet this might stem from the fact that environments were also explored by floors, of greater length than the height of vertical junctions (staircases). In Thibault et al. (2013), we have studied this potential bias with a learning and recognition experiment, for an object-in-place task, under carefully controlled conditions.

First, a virtual building was designed for the experiment as a repetition of rooms having the same height and length, connected by equivalent horizontal and vertical passages. Second, a group of 28 participants - the floor learners-learned the simulated space by watching a computer movie depicting floor-by-floor travel; similarly, 28 other participants - the column learners - observed the building according to a column-by-column route (i.e., travelling by elevators across floors). One landmark was visible in each visited room of the building. Third, we equated the sequence of landmarks encountered by floor-learning and columnlearning participants (we changed the positions of the landmarks in the virtual building for each group to achieve this).

We then observed the performances of the two groups in a recognition phase where the participants viewed a camera movement from one room to an adjacent one, either following a segment of the learned path (a familiar segment) or a shortcut (a novel segment). After being moved, they had to indicate the correct object (among three distractors) that was there during the learning phase.

Under these controlled conditions, we detected a positive influence of the learning route, by floors and also by columns, on spatial memory performances (in terms of accuracy and response times). We found that participants processed familiar segments of the learned path more accurately than novel ones, not only after floor learning $(80 \%$ correct answers in horizontal [familiar] trials vs. $63.5 \%$ in vertical [novel] trials) - which is in line with Montello and Pick's (1993) finding cited in section 3.3 of the target article (see the first two sentences of sect. 3.3, para. 5) but, crucially, also after column learning. Participants who learned the multilevel building by a column-bycolumn route performed better in the recognition of landmarks within a column compared to the recognition of landmarks across columns, that is, within a floor $(66 \%$ correct answers in vertical [familiar] trials vs. $48 \%$ in horizontal [novel] trials). ${ }^{1}$ This finding suggests a key role of the learning mode on the exploitation of spatial memory, which extends the findings reported by Jeffery et al.

As a complement, we here point out our own methodological limits:

1. Our work does not use a navigational task but an objectin-place one, which differs from the task considered by Jeffery et al. In our experiment, the participants viewed a camera movement - that is, they were not free to change their direction of movement and to update their navigation strategy.

2. Our experiment was conducted in a rectilinear environment: The participants cannot explore the $(x, y)$ plane, but only the $x$-axis. This can be a restricted case for the bicoded map model proposed by the authors.

NOTE

1. The chance level was equal to $25 \%$ correct answers.

\section{Anisotropy and polarization of space: Evidence from naïve optics and phenomenological psychophysics}

doi:10.1017/S0140525X13000332

Ivana Bianchi ${ }^{\mathrm{a}}$ and Marco Bertamini ${ }^{\mathrm{b}}$

${ }^{a}$ Department of Humanities, University of Macerata, 62100 Macerata, Italy; bSchool of Psychology, University of Liverpool, Liverpool L69 7ZA, United Kingdom.

ivana.bianchi@unimc.it

m.bertamini@liv.ac.uk

http://docenti.unimc.it/docenti/ivana-bianchi

http://www.liv.ac.uk/vp/marco.html 
Abstract: Additional evidence is presented concerning the anisotropy between vertical and horizontal encoding, which emerges from studies of human perception and cognition of space in plane mirror reflections. Moreover, it is suggested that the non-metric characteristic of polarization - that Jeffery et al. discuss with respect to gravity-is not limited to the vertical dimension.

As discussed by Jeffery et al., evidence for true volumetric coding is weak, and a bicoded map is the most likely encoding spatial scheme (at least in mammals). Moreover, allocentric encoding of the horizontal dimension is metric, whereas encoding of the vertical dimension is non-metric. Among the features of the vertical dimension is its polarization. In this commentary we focus on these two aspects (anisotropy and polarization). Jeffery et al. explicitly say that they confine their discussion to studies of navigation rather than spatial cognition. However, some parallels are strong. We draw attention to behavioral findings from naïve optics and phenomenological psychophysics. These data, respectively, (a) provide further evidence concerning the anisotropy between vertical and horizontal encoding of space in humans, and (b) suggest that polarization is a general characteristic of non-metric encoding of ecological space, not exclusive to the vertical dimension.

Many adults hold mistaken beliefs concerning the spatial behavior of reflections. For example, when adults imagine a person entering a room and walking parallel to a wall, they predict that the person will see his or her reflection in a mirror on the wall before it is possible (i.e., before being aligned with the mirror's edge - this has been called the "early error"). A minority also predict that the image will appear at the mirror's farther edge rather than at the nearer edge. The same mistake applies when moving in a real room, not just when predicting an event in a paper-and-pencil task (Croucher et al. 2002).

The early error is compatible with overestimation of what is visible in a mirror from left and right. In other words, people expect a cone of visibility for a given mirror, largely independent of the viewpoint of the observer and of distance (Bertamini et al. 2010; Bianchi \& Savardi 2012b). The second error (the location of the image) is compatible with the idea that the virtual world is spatially rotated around a vertical axis (Bertamini et al. 2003). However, an alternative explanation was found in later studies: The virtual space is thought of as allocentrically organized in an opposite way with respect to the real environment (Savardi et al. 2010).

These mistaken beliefs, which are similar in males and females, and in psychology and physics students, are confined to the horizontal plane. They disappear when vertical movements are considered. For instance, if the imagined character approaches the mirror by moving vertically - say, climbing up a rope parallel to the wall (Croucher et al. 2002) or moving in a glass lift (Bertamini et al. 2003) - people neither expect to see the reflection before reaching the near edge of the mirror, nor to see it appearing at the farther edge (i.e., opposite relative to the direction of the approach). Experience does not lead to better understanding of mirror reflections; on the contrary, adults make larger errors compared to children (Bertamini \& Wynne 2009).

Further evidence of a difference between transformations around horizontal and vertical axes comes from studies of perception of mirror reflections of the human body: Mirror-reflecting a picture or a real body around the horizontal axis - upside-down reversal-resulted in larger differences than did reflecting it around the vertical axis-left-right reversal (Bianchi \& Savardi 2008; Cornelis et al. 2009).

With respect to polarization, Jeffery et al. argue that this is a feature typical of the dimension orthogonal to the plane of locomotion (i.e., usually the vertical). They do not explicitly claim that it is confined only to this dimension, but they do not specify that polarization can also characterize the representation of the horizontal dimension (at least in humans). However, spatial polarization emerges as a key aspect in both dimensions from studies on the perception and cognition of space and objects in mirrors (Bianchi \& Savardi 2008; 2012b; Savardi et al.
2010), and it is a non-metric characteristic of direct experience of space that goes beyond mirror perception. This aspect of space has been emphasized in various anthropological (e.g., Brown \& Levinson 1993) and linguistic (e.g., Cruse 1986; Hill 1982) analyses of human cognition and in studies of memory errors in object recognition (Gregory \& McCloskey 2010). Only recently has its importance been recognized within what has been defined "phenomenological psychophysics" (Kubovy 2002; Kubovy \& Gepshtein 2003). The basic structure of phenomenological experiences of space and its geometry invariably manifests properties of polarization (Bianchi et al. 2011a; 2011b; Bianchi \& Savardi 2012a; Savardi \& Bianchi 2009). In addition, Stimulus-Response spatial compatibility can be thought of as a direct effect of an allocentrically polarized encoding of space in humans (Boyer et al. 2012; Chan \& Chan 2005; Roswarski \& Proctor 1996; Umiltà \& Nicoletti 1990).

Emerging from phenomenological psychophysics regarding polarization is the operationalization in metric and topological terms of spatial dimensions and the finding that these dimensions differ in the extent of the polarized area. This in turn depends on the extension of a central set of experiences perceived as neither one pole nor the other. For instance, the perception of an object as positioned "on top" of something (e.g., a flag on top of a mountain) is confined to one specific position; the same holds for "at the bottom." In between these two extremes there is a range of positions that are "neither on top, nor at the bottom." In contrast, in the dimension "in front of-behind" (e.g., two runners) there is only one state that is perceived as "neither in front, nor behind" (i.e., when the two runners are alongside). Similarly, in the dimension "ascending-descending" there is only one state that is perceived as "neither ascending, nor descending" (i.e., being level); all the other slants are recognized as gradations of ascending or of descending (Bianchi et al. 2011b; Bianchi et al. 2013). Furthermore, perhaps counter-intuitively, polarization is not to be thought of necessarily as a continuum: Ratings of high/low, large/small, wide/narrow, and long/short (dimensions relevant for navigation) applied to ecological objects or to spatial extensions do not behave as inverse measurements of the same continuum (Bianchi et al. 2011a).

\section{Navigating in a volumetric world: Metric encoding in the vertical axis of space}

\section{doi:10.1017/S0140525X13000344}

Theresa Burt de Perera, Robert Holbrook, Victoria Davis, Alex Kacelnik, and Tim Guilford

Department of Zoology, University of Oxford, Oxford OX1 3PS, United Kingdom.

Theresa.Burt@zoo.ox.ac.uk Robert.Holbrook@zoo.ox.ac.uk

Victoria.Davis@zoo.ox.ac.uk Alex.Kacelnik@zoo.ox.ac.uk\#

Tim.Guilford@zoo.ox.ac.uk

http://oxnav.zoo.ox.ac.uk/

Abstract: Animals navigate through three-dimensional environments, but we argue that the way they encode three-dimensional spatial information is shaped by how they use the vertical component of space. We agree with Jeffery et al. that the representation of three-dimensional space in vertebrates is probably bicoded (with separation of the plane of locomotion and its orthogonal axis), but we believe that their suggestion that the vertical axis is stored "contextually" (that is, not containing distance or direction metrics usable for novel computations) is unlikely, and as yet unsupported. We describe potential experimental protocols that could clarify these differences in opinion empirically.

Following behavioural and neurophysiological research on how three-dimensional space is encoded by animals, Jeffery et al. hypothesize that vertebrates represent space in a bicoded fashion, with spatial information about the plane of locomotion computed and represented separately from space in the 
orthogonal axis. We agree with this. Indeed, experimental evidence shows that fish that move freely through a volume represent space in two, separable, vertical and horizontal components, and not as an integrated three-dimensional unitary representation (Holbrook \& Burt de Perera 2009; 2011b). This is supported by studies on rats (Grobéty \& Schenk 1992a; Hayman et al. 2011; Jovalekic et al. 2011). However, this still leaves open the fundamental question of whether the vertical axis is represented "contextually" (defined as not available for novel quantitative processing) or metrically.

Jeffery et al. are ambiguous on this point, as their bicoding hypothesis can accommodate the vertical axis being represented metrically, but they favour the idea that it is likely to be encoded contextually, even for animals that move freely through three dimensions. They reasonably argue that contextual coding may free the animal's brain from complex trigonometric calculations in three dimensions, but this comes at the cost of constraining spatial computations. For instance, metric encoding allows the animal to know when it is getting close, is roughly where it should be, or has gone too far, and it allows the animal to use the relation between multiple landmarks, correcting for distance and perspective when computing novel trajectories. It has been argued that in the horizontal plane, ants integrate cross-modally metric information obtained from path integration with visual snapshots of landmarks (Lent et al. 2010; Mueller \& Wehner 2010). For animals that move freely in a volume, the same computational possibilities are important in the vertical axis.

Because metric information contains spatial relationships, the presence of metric representations can be expressed in how the pattern of error or generalization is distributed along the vertical axis. We offer two suggestions. First, we expect generalization along a metric vertical axis either side of significant locations, and for this "error" to obey Weber's law, displaying a roughly proportional relation between magnitude of the percept and accuracy (Foley \& Matlin 2010). Such generalization would not apply to contextual representations. This could be tested by measuring error in recall of a rewarded vertical position for an animal that can move freely in the vertical axis (and hence express that error). Second, generalization curves around a positive and a negative instance should interfere if the instances are close in a metric vertical axis, and this interference should take the behavioural form of a computable peak shift (Domjan 2009). So, if an animal is rewarded for visiting a place at a certain elevation, the presence of a punishing instance marginally above this should result in a predictable downwards displacement of response.

Whether an animal encodes the vertical axis metrically or not may be related to the animal's movement and whether it is surface bound with three degrees of freedom (forward-backward, left-right, and rotational (yaw) or moves with six degrees of freedom by the addition of up-down, roll and pitch. In flying or swimming animals, the equality in the freedom of movement in the horizontal and vertical components of space is likely to favour metrical scales for space in both the horizontal and vertical dimensions.

\section{Navigating through a volumetric world does not imply needing a full three-dimensional representation}

\section{doi:10.1017/S0140525X13000356}

\section{Claus-Christian Carbon and Vera M. Hesslinger}

Department of General Psychology and Methodology and Graduate School of Affective and Cognitive Sciences, University of Bamberg, D-96047 Bamberg, Bavaria, Germany.
Abstract: Jeffery et al. extensively and thoroughly describe how different species navigate through a three-dimensional environment. Undeniably, the world offers numerous three-dimensional opportunities. However, we argue that for most navigation tasks a two-dimensional representation is nevertheless sufficient, as physical conditions and limitations such as gravity, thermoclines, or layers of earth encountered in a specific situation provide the very elevation data the navigating individual needs.

As Jeffery et al. correctly note, most scientific efforts on largescale spatial relations have focused on two-dimensional settings while neglecting further, potentially important dimensions such as elevation, slant, and distortion. In theoretical terms, generating a more complete three-dimensional representation of the environment by integrating such information presumably enhances navigation accuracy. However, it is rather debatable whether this also leads to a significant improvement in actual navigation and localization performance in everyday tasks (Montello \& Pick 1993).

As a series of empirical works confronting (human) participants with navigation tasks has documented, specific deficits in the assessing of the azimuth angle, for instance, arise in multifloored/three-dimensional versus single-floored/two-dimensional settings (Hölscher et al. 2006; Thibault et al. 2013). In ecological contexts, offering a variety of orientation cues, humans are nevertheless able to actively navigate through three-dimensional environments without any problems. This might again indicate here that it is not obligatory to have access to a perfect cognitive three-dimensional representation of the environment. Furthermore, the mismatch of evidence provided by empirical studies and everyday experience might point to a lack of ecological validity in the paradigms commonly used to investigate the premises of actual navigation. This is partly due to laboratory and real-life navigation tasks requiring completely different, and sometimes even converse, strategies or behaviors. In a study by Carbon (2007), for example, participants were asked to estimate national large-scale distances as the crow flies - but what did they do in the end? Although they obviously used a consistent and steady strategy, as indicated by estimates being highly reliable as well as strongly correlated with the factual physical distances (cf. Montello 1991), they applied a strategy which differed entirely from the one specified in the instructions: Instead of linear distances, they used German Autobahn distances as the basis for their estimations, thus replacing the requested but unfamiliar mode (no human was ever found to behave like a bird) with one derived from everyday behavior - that is, from actually travelling these distances by car (instead of aircraft). Thus, everyday knowledge was found to be preferred over (artificial) task affordances, which is indeed reasonable since it is easier and more economical to do something on the basis of knowledge and familiar routines.

Let's get back to a point mentioned already and elaborate on why a complete three-dimensional representation of the environment is not obligatory for the majority of typical real-life navigation tasks. Surface-travelling species, for example, are limited to the surface they are travelling on; they might hop and dig from time to time, but they mainly orient themselves to the surface, thus inherently to the current elevation of the given structure of this surface. Basically, they navigate on an idealized plane. When directions of places are to be assessed within a single plane, azimuthal errors are relatively small (Montello \& Pick 1993), so navigation will be quite accurate. If sensory (e.g., visual) cues are additionally taken into account, it can be further tuned and optimized (Foo et al. 2005). Concerning navigation through three-dimensional environments, the ability of extracting and utilizing supplemental information provided by external or sensory cues turns out to be quite economic: Even a subject climbing a mountain can still locate its target destination (the peak) on an idealized two-dimensional map, provided some supplemental information on the elevation of this target is available. This information can be "gleaned," for instance, from the required expenditure of energy while climbing. Considering that most parts of the 
three-dimensional space can thus be reduced to a surface-map representation with sparse data requirements, a cognitive system encoding topographies in full three-dimensional coordinates seems rather unnecessary, as it would be too cost-intensive.

Regarding species such as flying insects, birds, or fish that move more freely through the third dimension, very similar navigation routines can be found (e.g., for honeybees: Lehrer 1994). Research has indeed revealed specific skills in communicating elevation (e.g., for fish: Holbrook \& Burt de Perera 2009; e.g., for stingless bees: Nieh \& Roubik 1998), and that elevation information can be highly relevant in some tasks (e.g., finding a bird's nest). Still, it is improbable that this information is fully integrated within a complete cognitive three-dimensional representation. From an information theory perspective, most parts of volumetric representations of real-world contexts would comprise a great number of "empty cells." Furthermore, reliable locations can hardly be imagined without any physical connection to the surface. A bird's nest, for example, may be situated in a treetop that is part of a tree that is itself solidly enrooted in the ground (i.e., the surface). Navigation requirements in the water, where spatial constraints are also obvious, are similar: Most relevant and reliable locations for hiding or for finding prey are near the bottom or the surface of the sea. For navigating through the sea, elevation information might be needed, but not necessarily in the form of a complete three-dimensional representation. Gibson's (1979) ecological approach offers a solid basis for reducing data for navigating tasks quite efficiently: Moving through a three-dimensional world itself provides important directly visual, acoustic, and proprioceptive cues (cf. Allen 1999), which help us to assess distances, elevations, and drifts of our movement trajectories both easily and accurately.

\section{Think local, act global: How do fragmented representations of space allow seamless navigation?}

\section{doi:10.1017/S0140525X13000368}

\section{Paul A. Dudchenko, ${ }^{\mathrm{a}, \mathrm{b}}$ Emma R. Wood, ${ }^{\mathrm{b}}$ and Roderick M. Grieves ${ }^{a, b}$}

a School of Natural Sciences, University of Stirling, Stirling, FK9 4LA United Kingdom; ${ }^{\mathrm{b}}$ Centre for Cognitive and Neural Systems, University of Edinburgh, Edinburgh EH8 9JZ, United Kingdom.

p.a.dudchenko@stir.ac.uk emma.wood@ed.ac.uk rmg3@stir.ac.uk www.memoryspace.mvm.ed.ac.uk

Abstract: In this commentary, we highlight a difficulty for metric navigation arising from recent data with grid and place cells: the integration of piecemeal representations of space in environments with repeated boundaries. Put simply, it is unclear how place and grid cells might provide a global representation of distance when their fields appear to represent repeated boundaries within an environment. One implication of this is that the capacity for spatial inferences may be limited.

The Appalachian Trail is a 2,184-mile-long footpath through the mountains of the eastern United States. Each year, approximately 2,000 "thru-hikers" attempt to backpack the trail's entire length, from Georgia to Maine, or vice versa. There are maps for each section of the trail, and hikers quickly learn that the most salient feature of these is the elevation profile. Miles are secondary to elevation change. When carrying a backpack, the amount of elevation gain is of paramount interest in planning the day's progress. Elevation has also been formalised in William Naismith's rule-of-thumb for estimating pedestrian distance: On flat ground, walkers usually cover 3 miles per hour, with an additional half hour added for each 1,000-foot elevation gain.

The consideration of three-dimensional spatial mapping by Jeffery et al. rightly brings attention to what thru-hikers and hill-walkers know firsthand - that elevation matters. But are distance and elevation represented in the same way, or in different ways? Jeffery et al., based on a review of behavioural and neurophysiological data, argue that three-dimensional space is represented in a bicoded (anistropic) internal map. Distances are represented metrically - likely by grid cells - whereas elevation is represented qualitatively, perhaps as a contextual cue. As an animal moves up, down, and across a complex environment, it may represent each surface as a map fragment, and these are linked in some way.

There is, however, a difficulty with map fragmentation, and it is not just in identifying how maps of different planes are linked. Rather, it is with the representations of space provided by grid, place, and to a lesser extent perhaps, head direction cells themselves. Put simply, while in an open space grid cells may encode distances, in more complex environments with similar, repeated geometric features, grid and place cells exhibit a fragmented encoding of space. Hence, the metric representation of space itself appears to be piecemeal. As we consider below, this may explain why demonstrations of novel spatial inference in the rat are a challenge.

For hippocampal place cells, accumulating evidence suggests that place fields are controlled by the local features of the environment. For example, Skaggs and McNaughton (1998) found that some place cells showed similar fields in each of two identical boxes. This result was replicated by Fuhs et al. (2005) in their same box orientation condition, and repeated fields have been found in up to four adjacent boxes (Spiers et al. 2009). These findings, and the demonstration that some place fields duplicate when a barrier is inserted into a square environment (Barry et al. 2006), provide clear support for the hypothesis that place fields are driven by the boundaries of the immediate environment (Hartley et al. 2000; O'Keefe \& Burgess 1996). Thus, an animal traversing an environment with repeated geometric features may experience a repeated, fragmented place cell representation. Indeed, the repeated place cell firing in the spiral maze observed by Hayman et al. (2011) may reflect this in the third dimension.

Fragmentary firing also occurs in medial entorhinal cortex grid cells. In an elegant experiment, Derdikman et al. (2009) showed that grid fields fragmented in a hairpin maze in which the animals ran in alternate directions through a series of alleyways. The firing of these cells appeared anchored to the start of each turn in a given direction for much of the distance in an alleyway, although firing near the end of the alley was anchored to the upcoming turn. In this same study, hippocampal place cells likewise exhibited repeated fields in different alleyways when the rat was running in the same direction. Thus, both grid cells and place cells exhibited a local, not global, representation of the environment. Presumably, this occurred even though the animal treated the environment as a whole.

What then of head direction cells? Here the situation is different. In the same hairpin maze, head direction cells were unchanged (i.e., they tended to fire in the same direction) compared to recording sessions in an open field (Whitlock \& Derdikman 2012). Remarkably, in cells that showed grid fields and directional tuning (conjunctive cells), grid fields fragmented in the hairpin maze, while directional firing was unaffected. The consistency of directional firing across maze compartments is in agreement with previous demonstrations that the preferred firing of head direction cells is maintained, with some error, across environments when the animal walks between them (Dudchenko \& Zinyuk 2005; Stackman et al. 2003; Taube \& Burton 1995).

On the face of the existing place and grid cell evidence, it is unclear how rodents solve distance problems in environments with repeated boundaries. This raises two possibilities. First, the distance map may become more global with repeated experience, though there is not strong evidence for this yet (Barry et al. 2006; Spiers et al. 2009). Second, it may be that rodents are not all that good at navigating multiple enclosure environments. For example, 
we have found that rats are surprisingly limited in their ability to take a novel shortcut between familiar environments (Grieves \& Dudchenko 2013). In short, their representations of the world may be local, and only incidentally global.

To return to Jeffery et al.'s main argument: The firing of grid and place cells in three-dimensional mazes is certainly consistent with a bicoded representation of space. Challenges that emerge from this view, and from the findings reviewed above, include identifying how global distance is represented when there is a change in elevation, and how it is represented when the environment contains repeated geometric features.

\section{Perceptual experience as a bridge between the retina and a bicoded cognitive map}

\author{
doi:10.1017/S0140525X1300037X
}

\section{Frank H. Durgin and Zhi Li \\ Department of Psychology, Swarthmore College, Swarthmore, PA 19081. fdurgin1@swarthmore.edurzhi.li.sh@gmail.com http://www.swarthmore.edu/SocSci/fdurgin1/publications.html}

Abstract: The bicoded cognitive maps described by Jeffery et al. are compared to metric perceptual representations. Systematic biases in perceptual experience of egocentric distance, height, and surface orientation may reflect information processing choices to retain information critical for immediate action (Durgin et al. 2010a). Different information processing goals (route planning vs. immediate action) require different metric information.

Jeffery et al. propose that the cognitive maps used by vertebrates are bicoded rather than fully volumetric, insofar as they represent a two-dimensional metric map in combination with a non-metric representation of the third dimension. In some cases, the twodimensional metric surface might be a vertical surface. Additionally, the maps are piecemeal. This is an important hypothesis that will help to focus future research in the field, but it raises questions concerning the relationship between metric representations in perception and those in cognition. Our commentary will propose possible links between metric perceptual experience and bicoded cognitive maps.

The visual world is normally understood as being sensed through a two-dimensional medium (e.g., the retina; pace Gibson 1979) that provides direct (angular) access to the vertical dimension and to the portion of the horizontal that is frontal to the observer. In contrast, the information most relevant for route planning might be said to be roughly along the line of sight itself - the horizontal depth axis, or the ground plane, which is highly compressed on the retina. A large number of visual (and non-visual) sources of information may be used to locate things in depth, but foremost among these, for locomotion, is information about angle of regard with respect to the ground surface (whether this is horizontal, slanted, or vertical) and eyeheight.

Relating the two-dimensional frontal retinal image to a cognitive metric surface map is an important achievement. The information evident to perceptual experience about surface layout in locomotor space includes information about surface inclination (i.e., slant relative to the gravitationally specified horizontal plane), as well as information about surface extent and direction. Over the past three decades, several competing claims about surface layout perception have been advanced, including affine models of depth-axis recovery (Wagner 1985), well-calibrated performance at egocentric distance tasks (Loomis et al. 1992), energy-based models of slant perception (Proffitt et al. 1995), intrinsic bias models of distance (Ooi \& He 2007), and our own angular scale expansion model of slant and distance (Durgin \& Li 2011; Hajnal et al. 2011; Li \& Durgin 2012).
The common observations that many of these models seek to address include perceptual facts that are superficially inconsistent with accurate metric spatial representations: Distances are underestimated (Foley et al. 2004; Kelly et al. 2004), surface slant relative to horizontal is overestimated ( $\mathrm{Li} \&$ D Durgin 2010; Proffitt et al. 1995), and object height is consistently overestimated relative to egocentric distance (Higashiyama \& Ueyama 1988; Li et al. 2011). Alongside these facts are the observations that angular variables, such as angular declination (or gaze declination) relative to visible or implied horizons, seem to control both perceptual experience and motor action (Loomis \& Philbeck 1999; Messing \& Durgin 2005; Ooi et al. 2001), emphasizing the importance of angular variables in the perceptual computation of egocentric distance.

Because the perceptual input to vision is specified in a polar coordinate system (the retinal "image," and its polar transformations afforded by eye, head, and body movements), a basic issue for relating the bicoded maps proposed by Jeffery et al. to the perceptual input is to understand the transformation from spherical coordinates to metric space. One argument that we have made in various forms is that metric spatial coding of distance might be incorrectly scaled in perception without producing a cost for action. Because our actions occur in the same perceptual space as our other perceptions (i.e., we see our actions; Powers 1973), they can be calibrated to whatever scaling we perceive. Metric representation is important to successful action (Loomis et al. 1992), but perceived egocentric distance can be mis-scaled as long as the scaling is stable and consistent.

We have observed that angular variables (including slant) seem to be coded in a way that exaggerates deviations from horizontal; this scaling could have the felicitous informational consequences of retaining greater coding precision while producing perceptual underestimation of egocentric ground distance (Durgin \& $\mathrm{Li}$ 2011), to which locomotor action can nonetheless be calibrated (Rieser et al. 1995). Believing that the resulting cognitive maps are metric does not require that perception also be metric in the same way (there is substantial evidence that egocentric horizontal extents appear shorter than frontal horizontal extents; see, e.g., Li et al. 2013), though it suggests that anisotropies in perceptual experience are overcome in cognitive maps. However, the notion that the vertical dimension is coded separately is intriguing and likely reflects a later transformation.

The perception of surface slant appears to require integration of vertical and horizontal metrics, and the systematic distortions evident in surface slant cannot be explained in detail merely by assuming that vertical scaling is expanded relative to horizontal scaling. Rather, the most elegant quantitative model of slant perception available suggests that perceived slant may represent the proportion of surface extent that is vertical (i.e., the sine of actual slant; see Durgin \& Li 2012). Importantly, the misperception of surface orientation shows constancy across changes in viewing direction (e.g., Durgin et al. 2010b) that implies that the coding of slant in perceptual experience is with respect to a gravitational (rather than an egocentric) reference frame, even when no horizontal ground surface is visible in the scene. It would therefore be of interest, based on the suggestion that the twodimensional cognitive map may sometimes be vertical, to determine how a vertical planar reference frame (e.g., a vertical navigation surface) affects the human interpretation of relative surface orientation.

The transformations between retinal input and cognitive maps probably involve an intermediate stage of perceptual representations of space that resembles neither. This perceptual stage clearly overcomes the superficial limitations of retinal images, yet it integrates vertical and horizontal (in the form of perceived surface orientation) in a way that cognitive maps may not. The selection of a two-dimensional metric representation is probably highly determined by information processing constraints in spatial cognition. Such ideas are also at the heart of scale expansion theory. 
Commentary/Jeffery et al.: Navigating in a three-dimensional world

\section{Learning to navigate in a three-dimensional world: From bees to primates}

doi:10.1017/S0140525X13000381

Adrian G. Dyer ${ }^{\mathrm{a}, \mathrm{b}}$ and Marcello G. P. Rosa ${ }^{\mathrm{b}}$

aschool of Media and Communication, RMIT University, Melbourne, VIC 3001, Australia; ' ${ }^{\circ}$ Department of Physiology, Monash University, Clayton, VIC 3800 , Australia.

adrian.dyer@rmit.edu.au@marcello.rosa@monash.edu http://www.rmit.edu.au/staff/adrian_dyer

http://www.med.monash.edu.au/physiology/staff/rosa.html

\begin{abstract}
We discuss the idea that environmental factors influence the neural mechanisms that evolved to enable navigation, and propose that a capacity to learn different spatial relationship rules through experience may contribute to bicoded processing. Recent experiments show that free-flying bees can learn abstract spatial relationships, and we propose that this could be combined with optic flow processing to enable threedimensional navigation.
\end{abstract}

Experiments that have investigated navigation in insects add considerably to the generality of the arguments presented by Jeffery et al., in revealing additional evidence for potential bicoded processing in animal brains. A contemporary point of interest in neuroscience is whether solving complex cognitive problems actually requires large mammalian brains (Chittka \& Niven 2009). In this regard, free-flying bees are an interesting model for understanding how visual systems deal with navigation in a three-dimensional world.

It is known that the mechanism by which both stingless bees (Melipona panamica) and honeybees (Apis mellifera) judge distance in the horizontal plane is visually driven by optical flow, where the velocity of angular image motion is integrated over time to enable a bee to estimate the flown distance with accuracy (Dacke \& Srinivasan 2007; Eckles et al. 2012). Interestingly, when honeybees are presented with the equivalent type of visual problem requiring judgment of distance using optic flow in the vertical plane, they solve this task with a much lower level of precision (Dacke \& Srinivasan 2007). We argue that the relative accuracy of navigation in the horizontal and vertical dimensions makes sense in terms of normal ecological imperatives, and that brain plasticity can be recruited to facilitate robust bicoded processing if required.

Studies on honeybees suggest that visual processing of optic flow is dominated by information impinging on the ventral visual field (Dacke \& Srinivasan 2007), which makes ecological sense for an animal that predominantly flies in the horizontal plane when foraging for nutrition. In contrast, the stingless bee operates in dense tropical environments where flower resources are typically scattered horizontally and throughout a range of vertical heights of up to 40 metres in the forest canopy. These stingless bees have been shown to be equally proficient at gauging distance in both the horizontal and vertical planes, potentially using optic flow mechanisms in both ventral and lateral visual fields (Eckles et al. 2012). Nonetheless, honeybees do sometimes also have to operate in complex three-dimensional environments such as tropical forests. How might they deal with the complexity of reliable orientation in the vertical plane? We suggest below that honeybees are able to use information other than optic flow, and that their brains can learn to combine different visual perceptions to solve novel problems, in a manner consistent with the bicoded hypothesis.

Whilst honeybees do communicate horizontal direction and distance to hive mates through a symbolic dance communication language, this does not reliably communicate elevation (Dacke \& Srinivasan 2007). It is therefore likely that individual foragers must learn through their own experience to determine vertical components of their three-dimensional world. One possible solution to this problem is relationship learning - estimating vertical position by understanding the relative relationships, such as above/below, between different elemental features in the environment. The capacity to process such relationship rules is not innate for a brain. Three-months-old human babies do not show evidence of relationship processing, although by 6 months of age this capacity has developed (Quinn et al. 2002). Other adult primates, such as capuchin monkeys (Cebus apella), also show a capacity for solving problems requiring the learning of above/ below rules (Spinozzi et al. 2004). Recent work in honeybees shows that while their brains do not innately code spatial relationship rules, individual free-flying bees can learn such relationships through visual experience, including the reliable processing of above/below (Avarguès-Weber et al. 2012).

The relationship rule processing mechanism observed in honeybees would thus give the potential for experienced individuals to forage in complex environments with acquired knowledge about relative vertical positions between biologically plausible objects, such as flowers and tree limbs (Chittka \& Jensen 2011; Dyer et al. 2008). Could "vertical knowledge" then be combined with the perception of optic flow in the horizontal plane, to give a true bicoded perception of three-dimensional space? We believe so. The work on how honeybees process complex visual relationship rules, including above/below and same/different, suggests that there is also a capacity to learn simultaneously two separate rules or types of visual information, and then combine this acquired knowledge to solve novel problems (Avarguès-Weber et al. 2012). Thus, although the honeybee appears to process horizontal and vertical optic flows as separate signals (Dacke \& Srinivasan 2007), it does appear that their brains have the capacity to combine multiple sources of sensory perception and other spatial cues to make novel decisions in complex environments.

In summary, learning to build concepts to process multiple dimensions of three-dimensional navigation using a bicoded system as hypothesised by Jeffery et al. may represent a more general biological capacity, which likely extends to relatively simple brains such as those of insects. Further, we suggest that the opportunity for a brain of an individual to learn complex tasks through experience is critical to revealing the true behavioural capabilities in animals, irrespective of the relative number of neurons and synapses involved.

\section{Spatial language as a window on representations of three-dimensional space}

doi:10.1017/S0140525X13000393

\author{
Kevin J. Holmes ${ }^{a}$ and Phillip Wolff ${ }^{b}$ \\ ${ }^{a}$ Department of Linguistics, University of California-Berkeley, Berkeley, CA \\ 94720; ${ }^{\mathrm{b}}$ Department of Psychology, Emory University, Atlanta, GA 30322. \\ kjholmes@berkeley.edu pwolff@emory.edu \\ http://userwww.service.emory.edu/ kholme2/ \\ http://userwww.service.emory.edu/ pwolff/CLSLab.htm
}

Abstract: Recent research investigating the language-thought interface in the spatial domain points to representations of the horizontal and vertical dimensions that closely resemble those posited by Jeffery et al. However, the findings suggest that such representations, rather than being tied to navigation, may instead reflect more general properties of the perception of space.

Jeffery et al. propose that bicoded representations may support the encoding of three-dimensional space in a wide range of species, including non-surface-travelling animals. Only humans, however, have the ability to draw on their representations of space to talk about their spatial experience. Here we highlight the potential of spatial language-not traditionally considered in the study of navigation through space - to provide insight into the nature of nonlinguistic spatial representation. In particular, we suggest that recent research on spatial language, spatial 
cognition, and the relationship between the two offers an unexpected source of evidence, albeit indirect, for the kinds of representations posited by Jeffery et al. Such evidence raises the possibility that bicoded representations may support spatial cognition even beyond navigational contexts.

There are several striking parallels between Jeffery et al.'s account of spatial representation and the semantics of spatial language. Jeffery et al. argue that animals represent the vertical dimension in a qualitatively different manner than they do the two horizontal dimensions, in large part due to differences in locomotive experience. This distinction between vertical and horizontal is also evident in spatial language. Clark (1973) noted that English spatial terms rely on three primary planes of reference, one defined by ground level (dividing above from below) and the other two defined by canonical body orientation (dividing front from back and left from right). In a similar vein, Landau and Jackendoff (1993) pointed to axial structure (e.g., the vertical and horizontal axes) as a key property encoded by spatial prepositions, which otherwise tend to omit much perceptual detail (see also Holmes \& Wolff 2013a). More recently, Holmes (2012; Holmes \& Wolff 2013b) examined the semantic organization of the spatial domain by asking native English speakers to sort a comprehensive inventory of spatial prepositions into groups based on the similarity of their meanings. Using several dimensionality reduction methods to analyze the sorting data, including multidimensional scaling and hierarchical clustering, Holmes found that the first major cut of the domain was between vertical terms (e.g., above, below, on top of, under) and all other prepositions; terms referring to the left-right and front-back axes (e.g., to the left of, to the right of, in front of, behind) tended to cluster together. These findings suggest that the vertical-horizontal distinction may be semantically, and perhaps conceptually, privileged.

Holmes's (2012) findings are also consistent with Jeffery et al.'s claims about the properties that distinguish horizontal from vertical representations. Jeffery et al. propose that horizontal representations are relatively fine-grained, whereas vertical representations are coarser and nonmetric in nature. In Holmes's study, prepositions encoding distance information (e.g., near, far from) clustered exclusively with horizontal terms, implying that metric properties are more associated with the horizontal dimensions than the vertical. Further, vertical terms divided into discrete subcategories of "above" and "below" relations, but horizontal terms did not; English speakers regarded to the left of and to the right of as essentially equivalent in meaning. Perhaps most intriguingly, Holmes found that the semantic differences among the dimensions were mirrored by corresponding differences in how spatial relations are processed in nonlinguistic contexts (see also Franklin \& Tversky 1990). When presented with visual stimuli depicting spatial relations between objects (e.g., a bird above, below, to the left of, or to the right of an airplane), participants were faster to discriminate an "above" relation from a "below" relation than two different exemplars of an "above" relation - but only in the right visual field, consistent with the view that the left hemisphere is specialized for categorical processing (Kosslyn et al. 1989). Though observed for vertical relations, this effect of lateralized categorical perception, demonstrated previously for color (Gilbert et al. 2006) and objects (Holmes \& Wolff 2012), was entirely absent in the case of horizontal relations: Participants were just as fast to discriminate two different exemplars of "left" as they were to discriminate "left" from "right." That the vertical dimension was perceived categorically but the horizontal dimension was not suggests differences in how the mind carves up spatial information along different axes. In characterizing the nature of the bicoded cognitive map, Jeffery et al. use color merely as a way of illustrating the nonmetric property of vertical representations, but such an analogy seems particularly fitting: Whereas the vertical axis may be represented in the same way that we see a rainbow as forming discrete units, the horizontal axis may be represented more like color actually presents itself, namely as a continuous gradient.
Together, the findings reviewed above tell a story about spatial representation that is, in many respects, similar to that proposed by Jeffery et al. in the target article. However, such findings suggest an alternative explanation for the many differences observed between the horizontal and vertical dimensions. Early in the target article, Jeffery et al. briefly distinguish between spatial navigation and spatial perception more generally, implying that the representations supporting navigation may not extend to other spatial contexts. But given the parallels between spatial language and the representations implicated by Jeffery et al.'s account, and the fact that spatial terms often refer to static spatial configurations rather than motion through space, bicoded representations may constitute a more general property of spatial perception, rather than being specifically tied to navigation. This possibility could be examined in future research on the representation of three-dimensional space. More broadly, our observations suggest a role for research on the language-thought interface in informing accounts of cognitive abilities ostensibly unrelated to language, lending support to the enduring maxim that language is a window into the mind (Pinker 2007).

\section{Multi-floor buildings and human wayfinding cognition}

\author{
doi:10.1017/S0140525X1300040X
}

Christoph Hölscher, Simon Büchner, and Gerhard Strube

Center for Cognitive Science \& SFB/TR8 Spatial Cognition, University of Freiburg, 79098 Freiburg, Germany.

hoelsch@cognition.uni-freiburg.de simon.buechner@cognition.uni-

freiburg.de strube@cognition.uni-freiburg.de

http://portal.uni-freiburg.de/cognition/members/hoelscher

http://portal.uni-freiburg.de/cognition/members/buechner

http://portal.uni-freiburg.de/cognition/members/strube

Abstract: Multilevel wayfinding research in environmental psychology and architecture exhibits a strong compatibility with Jeffery et al.'s "bicoded" representation of space. We identify a need for capturing verticality in spatial analysis techniques such as space syntax and argue for investigating inter-individual differences in the ability to mentally integrate the cognitive maps of separate floors in buildings.

Our commentary focuses on navigating multilayer environments and extends Jeffery et al.'s view to an architectural and environmental psychology perspective.

The functions of buildings are generally organized horizontally, probably reflecting the constraints that humans encounter: A horizontal plane is neutral to the axis of gravity and allows for stable walking, sitting, and storing of objects. Humans and buildings "inhabit" the same "two-dimensional" ecological niche, and buildings stack floors on top of one another. As a consequence, the structure of typical buildings is highly compatible with the "bicoded" representation: Whereas the horizontal plane is continuous (albeit subdivided by corridors and partitions) and in line with the floors, the vertical axis is discontinuous and discretized; that is, floors are on top of one another, with only local connections via stairs or elevators. Unless one has a view along a multi-storey atrium, the vertical dimension is visually limited to the current or directly adjacent floor. Verticality is presented as "contextual," at ordinal rather than metric scale, and perceived indirectly or derived by inference processes.

Tlauka et al. (2007) describe a systematic bias in vertical pointing between floors. Across several studies in our group we have observed a pattern in the process of pointing that links to the bicoded representation: Pointing appears to be based on categorical and discretized rather than continuous information, visible in smooth horizontal pointing and stepwise vertical pointing. Participants report counting floors, rather than making spontaneous 
judgments (Hölscher et al. 2009). This is further illustrated by longer response times for the vertical component of the pointing gesture.

Environmental researchers such as Weisman (1981) and Carlson et al. (2010) differentiate between four types of environmental characteristics that impact navigation in buildings: (1) visual access to decision points, (2) architectural differentiation (the likelihood of confusing visual scenes), (3) layout geometry, and (4) signage. These features can be seen in the light of the bicoded representation, with vertical integration as the central challenge. Several studies (e.g., Soeda et al. 1997) illustrate that multilevel public buildings are inherently difficult to navigate, and wayfinding complexity can be traced to mentally linking individual floors of the building. Participants expect a consistent corridor layout across floors and are disoriented by layout differences between floors. Buildings in which the floors are partially horizontally offset from one another, such as the Seattle Public Library (by architect Rem Koolhaas; Carlson et al. 2010), provide an additional challenge for the mental alignment of corresponding locations across floors.

Vertical connections form salient elements in the mental representation of the building and are usually limited in number. Level changes are a key source of disorientation about one's heading and position in a building (Hölscher et al. 2006), especially when a staircase is offset from the main corridor axis, enclosed by walls, or requires many turns. An atrium in the center of a building can provide visual access between floors that helps people correctly align floors in their memory representations (Peponis et al. 1990), especially when the staircase/escalator/elevator is visually linked to this vertical core. Views to the outside with a distinctive global landmark can further support integration of cognitive maps between floors.

The architectural community has developed spatial analysis methods to predict human movement patterns and cognitive representations, namely "space syntax" (Conroy Dalton 2005; Hillier \& Hanson 1984) and "isovist” approaches (Benedikt 1979; Wiener et al. 2012), capturing visual access and layout complexity alike. It is noteworthy that these approaches concentrate on single-floor environments and that current space syntax analysis treats multilevel connections only in a simplified fashion (Hölscher et al. 2012). While this reflects a correspondence with the bicoded view, more fine-grained analysis of vertical connectors in building analysis is called for.

Human indoor navigation is strongly driven by the semantics of floor layout and expectations about the positioning of meaningful/ typical destinations and landmark objects (Frankenstein et al. 2010), as well as by expectations of regularity, good form, and closure (Montello 2005). Human navigation is further guided by symbolic input like signage and maps. Hölscher et al. (2007) have shown that signage and cross-sectional maps explicitly designed to highlight multilevel relations and connections between floors help to overcome vertical disorientation, making first-time visitors almost as efficient in wayfinding as a benchmark group of frequent visitors.

Based on their experience with typical buildings, humans develop specific strategies for handling vertical layouts (e.g., central-point or floor strategies; Hölscher et al. 2006). Jeffery et al. refer to Büchner et al.'s (2007) finding that more people preferred a horizontal-first route. This could be partly due to the fact that the horizontal distances were longer than the vertical here (as in many buildings) and that people tend to follow the longest line of sight (Conroy Dalton 2003; Wiener et al. 2012). Furthermore, people adapt their strategies to properties of the building; the aforementioned floor strategy can then be replaced by a "lineof-sight" strategy, depending on the connections in a complex building (Hölscher et al. 2009).

Finally, we wish to point to the need for understanding the role of inter-individual differences in handling verticality, along the distinction of route versus survey knowledge, and the degree to which humans are able to coherently integrate cognitive maps of stacked floors. Studies cited by Jeffery et al., as well as our own, are compatible with separate, planar representations of spatial information. However, a subset of participants in our experiments consistently reports imagining the environment from an external perspective, with walls and floors like glass ("a glass doll house"). This appears to require a consistent representation of the vertical dimension, and these people tend to report allocentric rather than egocentric orientation strategies, as well as high levels of spatial abilities. Jeffery et al. state that investigating multi-floor environments alone is insufficient to identify to what degree surface-dwelling animals can build true volumetric mental maps. Virtual-reality simulations of such environments for example, tilting a building layout by 90 degrees or comparing multilevel navigation to "vertical floating" along tunnels - provide useful extensions in this direction. In studies currently under way, we focus on the role of individual differences in how the vertical component is integrated.

\section{Applying the bicoded spatial model to nonhuman primates in an arboreal multilayer environment}

\author{
doi:10.1017/S0140525X13000411
}

\begin{abstract}
Allison M. Howard and Dorothy M. Fragaszy
Department of Psychology, University of Georgia, Athens, GA 30602-3013. allisonmariehoward@gmail.com doree@uga.edu http://psychology.uga.edu/primate/
\end{abstract}

Abstract: Applying the framework proposed by Jeffery et al. to nonhuman primates moving in multilayer arboreal and terrestrial environments, we see that these animals must generate a mosaic of many bicoded spaces in order to move efficiently and safely through their habitat. Terrestrial light detection and ranging (LiDAR) technology and three-dimensional modelling of canopy movement may permit testing of Jeffery et al.'s framework in natural environments.

Jeffery et al. propose that a bicoded representation of threedimensional space, in which horizontal and vertical dimensions are encoded in fundamentally different ways (i.e., metric and non-metric), is common to all vertebrate animals. Although bicoded spatial representation has implications for animals moving in all substrates, this commentary focuses on how the theoretical framework outlined by Jeffery et al. might be applied to nonhuman primates moving in natural multilayer environments and what techniques might be applied to this problem.

The neural evidence upon which Jeffery et al. base their conclusions comes largely from rats habitually moving within single layers of space or in multilayered, compartmentalized space (e.g., tunnels). The authors also describe animals that move in volumetric space (e.g., fish, birds) and the need for data regarding how these animals represent space in three dimensions. Applying Jeffery et al.'s framework to arboreal/terrestrial nonhuman primates, we find that the space in which these animals move presents significant challenges for generating spatial representation mosaics which Jeffery et al.'s examples do not habitually encounter. A nonhuman primate that moves both on the ground and in trees (e.g., chimpanzees, capuchin monkeys, macaques) is presented with the option of travel on substrates that may occur at a great variety of distances, angles, and heights from the horizontal plane of the animal's location at any given moment. Vertical, horizontal, and intermediately angled arboreal substrates comingle and contact substrates from neighboring trees. The animal must accurately estimate distance to cross open spaces by leaping or brachiation. These locomotor patterns are associated not only with horizontal, but also vertical displacements of the animal (e.g., Channon et al. 2010). Considering Jeffery et al.'s 
Figure 12 in the context of a primate travelling in the trees, we might visualize similar arboreal bicoded map fragments that occur along numerous branches in many directions and extending into various distances from the central tree trunk. These bicoded map fragments meet and join fragments from neighboring trees, forming a web of metrically mapped spaces that, with increasing density of branches and variation in the branch angles, will approach metrically mapping both horizontal and vertical space (in the canonical orientation sense), assuming that the bicoded fragments are accurately joined in a mosaic.

Landscapes within which animals move consist of both vertical and horizontal components that animals act upon. Landscape components such as topography have an impact on the manner in which animals move through their environments (e.g., Mandel et al. 2008). Animals moving primarily through a single layer of their environment (e.g., large grazing animals) are impacted by elevation, the one vertical component of the landscape upon which they move (Bennett \& Tang 2006). Because moving uphill requires greater energy expenditure than moving on level ground (Taylor \& Caldwell 1972), terrestrial animals may make changes in the horizontal nature of their movements in order to avoid a given area due to its vertical character (i.e., detouring around steep slopes). In contrast, animals that move in multilayer environments contend with elevation as well as other additional vertical components to their movement decisions (i.e., substrate height). For example, nonhuman primates and other vertebrates locomote in multilayer environments with a combination of arboreal and terrestrial substrates. The varying slopes of the multitude of potential substrates (i.e., branches, tree trunks, and terrestrial surfaces) and the locations to which these substrates convey present these animals with numerous options for movement. These options also allow the animal to exert greater control over the vertical component of its movement decisions. For example, continuous canopy forest would allow arboreal primates and other quadrupedal animals that move through arboreal habitats to travel at a constant height, minimizing vertical displacement, while the elevation of the forest floor rises and falls. We maintain that the use of non-compartmentalized multilayer environments requires a representation of space that is sufficiently accurate to allow for movement decisions in the vertical and horizontal dimensions as well as precise aerial swinging/leaping to distal substrates. Logistically, this spatial model may be hypothesized to include, at a minimum, precise heuristics regarding where, when, and how to swing or leap, or perhaps even a metric component of the vertical dimension.

In studies of the movement of nonhuman primates in multilayer environments, movement observations are frequently simplified for their analysis. Actual three-dimensional animal movements are reduced to two-dimensional displacements across a planar surface (Janson 1998; 2007; Normand \& Boesch 2009; Noser \& Byrne 2007b; Sueur 2011; Valero \& Byrne 2007). Some prior studies have incorporated the vertical dimension of movement in discussions of nonhuman primate movement patterns by linking elevation to visibility of resource sites (e.g., Di Fiore \& Suarez 2007; Noser \& Byrne 2007a). Draping the movements of nonhuman primates onto a digital elevation model of their habitat allows us to consider the energetic and viewpoint effects resulting from the vertical component of movement on a terrestrial substrate (e.g., Howard et al. 2012). However, the greater vertical complexity of moving through multilayer environments (e.g., arboreal and terrestrial) and its effects on adaptive movement choice are not considered using this technique.

One technique that may accurately represent the arboreal substrates upon which nonhuman primates move is Light Detection and Ranging (LiDAR) technology. Terrestrial LiDAR is threedimensional laser scanning that generates a hemispherical point cloud representing the returns of laser pulses from a groundbased vantage point (Beraldin et al. 2010). This high-density point cloud can be used in forest inventories and in measuring the detailed geometric characteristics of trees (Maas 2010).
Point clouds can be used to generate three-dimensional models of the canopy through which animals move. The use of this technique would allow researchers to catalog all possible substrates on a tree or group of trees, estimate the energetic costs of movement on those substrates, and even model animals' movement through a multilayer canopy based on heuristics or metric knowledge of vertical and horizontal spatial components of their environment. In this way, the framework proposed by Jeffery and colleagues may be tested against movements of animals in natural environments.

\section{The complex interplay between three- dimensional egocentric and allocentric spatial representation}

\author{
doi:10.1017/S0140525X13000423
}

\section{David M. Kaplan \\ Department of Anatomy and Neurobiology, Washington University School of Medicine, St. Louis, MO 63110. \\ kaplan@eye-hand.wustl.edu}

Abstract: Jeffery et al. characterize the egocentric/allocentric distinction as discrete. But paradoxically, much of the neural and behavioral evidence they adduce undermines a discrete distinction. More strikingly, their positive proposal-the bicoded map hypothesis - reflects a more complex interplay between egocentric and allocentric coding than they acknowledge. Properly interpreted, their proposal about threedimensional spatial representation contributes to recent work on embodied cognition.

The efforts of Jeffery et al. to synthesize and provide an overarching theoretical interpretation for the literature on two-dimensional and three-dimensional spatial representation will undoubtedly prove useful to the field. Their focus on 3D space also highlights the importance of investigating navigation performance in more complex, ecologically valid task environments. Despite these strengths, the authors fall prey to a common confusion involving the central egocentric/allocentric distinction.

At the outset, the authors flag this familiar distinction to emphasize how the "focus of the present article is on the allocentric encoding of the space that is being moved through" (target article, sect. 2, para. 1). Although a discrete distinction is widely assumed in the literature, and the authors imply it is incidental to the main goals of the article and hence can be glossed over, taking it at face value is problematic for two reasons. First, much of the neural and behavioral evidence the authors survey undermines a clean division. Second, and more strikingly, their positive proposal - the bicoded map hypothesis, in which different encoding schemes are employed for horizontal versus vertical space, yet both are referenced to the organism's plane of locomotion - clearly reflects a more complex interplay between egocentric and allocentric spatial representation than the authors explicitly acknowledge.

Egocentric and allocentric representations are primarily distinguished by the different reference frames they employ. Reference frames specify locations in terms of distances along two (or three) perpendicular axes spanning out from an intersection point at the origin. Egocentric representations specify locations relative to a reference frame centered on a body axis, such as the midline, or on a body part of the organism (e.g., " $20 \mathrm{~cm}$ to the right of my right hand"). Allocentric representations encode locations relative to a reference frame centered on some environmental feature or object (or set of these), and independently of the organism's own orientation or possibly even position (e.g., “33ㅇ $151^{\circ} \mathrm{E}$ ”). Framed in this way, the key difference underlying the egocentric/allocentric distinction concerns the origin or center of the reference frame for the spatial representation, a point which is clearly 
reflected etymologically in the terms "egocentric" and "allocentric." Nevertheless, characterizing a spatial reference frame involves more than just specifying the origin. Axis orientation and a metric for assessing distances along these axes must also be determined. This gives rise to another facet of the egocentric/allocentric distinction that has been largely ignored in the literature (for an exception, see Grush 2007).

There are at least two senses in which a spatial representation may be egocentric (or allocentric). As canvassed above, a representation can be egocentric if the origin of the reference frame is anchored to the observer's location. But a spatial representation can also be egocentric if the alignment or orientation of the reference frame axes is itself dependent on properties of the organism (independently of whether the origin is fixed to the organism or not). Consider how bodily features such as the dorsal/ventral axis of the head might serve to determine the up/ down axis for certain egocentric spatial representations used in early vision (Pouget et al. 1993), or how the body midline might serve to orient the left/right axis for spatial representations employed in reach planning (Pouget \& Sejnowski 1997). These representations are plausible candidates for being egocentric in both senses. Although these two kinds of egocentricity can go hand in hand, this is not necessary. Importantly, allocentric spatial representations are subject to an equivalent analysis, such that a representation may be allocentric with respect to its origin, axes, or both.

A more nuanced egocentric/allocentric distinction facilitates the identification of a richer representational taxonomy including hybrid cases incorporating both egocentric and allocentric elements as building blocks. For example, consider a hybrid representation in which locations are specified relative to an egocentric origin, but where the horizontal and vertical axes are aligned with respect to some environmental feature such as the Earth's magnetic field. The axes defined by the cardinal directions are allocentric, as they bear no direct relationship to any egocentrically defined axes and are invariant across changes in orientation and position of the subject.

This is important because the authors' bicoded map hypothesis posits a hybrid spatial representation, incorporating an allocentrically defined origin and egocentrically defined axes. According to the hypothesis, different encoding schemes (metric vs. nonmetric) are used for the representation of horizontal and vertical space in the hippocampus and medial entorhinal cortex (MEC). Importantly, these schemes are not referenced to horizontal and vertical per se (i.e., gravitationally defined earth-horizontal and earth-vertical) as might be expected based on previous navigation studies employing two-dimensional arenas. Instead, studies conducted by the authors using three-dimensional environments (Hayman et al. 2011) and by other groups using microgravity conditions (Knierim et al. 2000), in which egocentric and allocentric axes can be explicitly dissociated, indicate that the "horizontal" axis is defined by the organism's canonical orientation during normal locomotor behavior and the axis orthogonal to this defines "vertical." If correct, this organism-dependent axial alignment makes the spatial representation under consideration egocentric in the second sense outlined above. Nevertheless, the metrically coded (horizontal) portion of this map, implemented by assemblies of hippocampal place cells and entorhinal grid cells, also possesses characteristic features of an allocentric representation, as its reference frame is anchored to the external environment and does not depend on the organism's own location. The bicoded map is a hybrid spatial representation.

When the bicoded map hypothesis is interpreted as has been suggested here, its link to recent work in the field of embodied cognition becomes evident. Embodied cognition researchers have long sought to reveal how physical embodiment and motor behavioral capacities shape and constrain our ability to represent space. Until now, findings in support of this link were restricted to how organisms represent their local workspace. If Jeffery et al. are right, evidence is emerging for how an organism's motor behavioral repertoire may also influence its representation of large-scale, navigable space.

\section{The planar mosaic fails to account for spatially directed action}

\author{
doi:10.1017/S0140525X13000435
}

Roberta L. Klatzky ${ }^{\mathrm{a}}$ and Nicholas A. Giudice ${ }^{\mathrm{b}}$

${ }^{a}$ Department of Psychology, Carnegie Mellon University, Pittsburgh, PA 15213; 'S Spatial Informatics Program, School of Computing and Information Science, University of Maine, Orono, ME 04469-5711.

klatzky@cmu.edu nicholas.giudice@maine.edu http://www.psy.cmu.edu/people/klatzky.html http://spatial.umaine.edu/faculty/giudice/

Abstract: Humans' spatial representations enable navigation and reaching to targets above the ground plane, even without direct perceptual support. Such abilities are inconsistent with an impoverished representation of the third dimension. Features that differentiate humans from most terrestrial animals, including raised eye height and arms dedicated to manipulation rather than locomotion, have led to robust metric representations of volumetric space.

Consider some human capabilities for actions directed at spatial targets at varying distances above the ground plane: at an extreme, snagging a fly ball on the run or pole vaulting; at the mundane level, turning on the wall switch or stepping over an obstacle on the ground. These actions are accurate and precise; yet they generally are not performed under closed-loop control that would free us from metric demands. It seems unlikely that the planar mosaic representation proposed by Jeffery et al. - where the third dimension is not only non-metric, but unstable - could support their execution.

How do we resolve the disparity between what would be possible under a non-metric representation of space and what people can actually do? One avenue toward resolution is to say, "Oh, but Jeffery et al. are not referring to those types of behaviors." But what, then, differentiates the behaviors ostensibly governed by the planar mosaic from human spatially directed actions such as pointing, reaching, over-stepping, and making contact?

For one thing, actions such as catching balls and reaching for targets on a wall result from characteristics of human perception and action that most other terrestrial mammals do not share. Humans are "ecologically three-dimensional" to a high degree. Our raised eyes provide a perspective view of the terrain where we might travel, within a volumetric context so vast it has been called "vista space" (Cutting \& Vishton 1995). Although not without error, our representation of height variation across environmental undulations is simply not possible for animals whose sense organs remain close to the ground during navigation. Humans differ as well from rodents and ants by having arms: limbs that are used not for locomotion (beyond infancy), but rather to reach and manipulate objects above the ground.

Spatially directed actions also potentially differ from terrestrial navigation in that the corresponding motor commands must specify the disposition of the entire organism in volumetric space - not only its location in point coordinates, but limb postures and joint angles. Perhaps this provides an avenue of reconciliation with the planar mosaic representation. Actions directed toward targets with particular metric relationships to the body may be designated as egocentric. Hence, they would lie specifically outside the scope of the current theory, which restricts itself to allocentric (environmentally referred) representations. This exclusion of metrically constrained behaviors from consideration is undermined, however, by the intrinsic ambiguity of frames of reference (Klatzky 1998). Humans flexibly compute transformations between self-referred and environmentally referred frames, even within a single task (Avraamides et al. 2004). 
Lacking reliable behavioral or neural signatures that would allow us to designate actions as egocentric on the basis of their metric demands, it seems inappropriate simply to exclude them from a theory of volumetric spatial representation.

But wait - there is another feature of reaching, jumping, catching, and so on, that might render such behaviors distinct from those guided by a volumetric mosaic. Arguably, these behaviors are supported by perception, rather than representational abstractions. This argument might work if perceptually guided actions are somehow different from those guided by something called "representation," presumably including navigation. However, a position to the contrary has been put forward by Jack Loomis and the present authors, along with collaborators (for review, see Loomis et al. 2013). Our proposal stems from the fundamental idea that the perceptual system functions to create representations, and it is these representations that guide action.

We have used the term "spatial image" to refer to a particular type of representation that can support behaviors such as navigation and reaching, even when sensory systems no longer provide data about the physical world. For example, when a sound is emitted and then ceases, a spatial image of the sound's location still remains to support navigation (Loomis et al. 2002). The spatial image makes possible not only orienting and direct locomotion toward the target, but also spatial updating by means of path integration.

Importantly for present concerns, we have recently shown that the spatial image is three-dimensional and can be formed by actions with the arm, as well as by vision (Giudice et al. 2013). Subjects in our experiments formed representations of locations in volumetric space that they viewed, touched directly, or probed with a stick. They then walked without vision by an indirect path, requiring spatial updating, and touched the target at the appropriate height. Localization was not only accurate, but minimally affected by how the target representation was formed: The mean height error (signed distance between target and response height) was only $1,9,7$, and $3 \mathrm{~cm}$ for targets originally explored with the hand, long pole, short pole, and vision, respectively. Precision, as measured by variability around the response location, was also little affected by mode of exploration.

These findings demonstrate several pertinent points. First, fully three-dimensional spatial representations were formed with high accuracy. Second, those representations conveyed metric data sufficient to support navigation, even in the absence of vision - that is, with perceptually guided homing precluded and spatial updating as a requirement. Third, the spatial image afforded action directed toward the object with the arms, as well as locomotion.

We have posited that human action capabilities require a metric representation of volumetric space that seems incompatible with the claim that terrestrial mammals are restricted to an impoverished representation of the third dimension, in the form of a loosely bound mosaic of planar maps. Although we have focused on humans, we see no reason not to extend these arguments to primates more generally, and we note that other mammals, such as squirrels and cats, routinely leap and jump to vertical target locations. We concede, however, the potential for constraints on human spatial representation. In particular, when our circumstances become more like those of animals confined to the ground plane (e.g., when travelling in a car), our cognitive maps, too, may provide only a local orientation relative to the direction of travel.

\section{Monkeys in space: Primate neural data suggest volumetric representations}

\section{doi:10.1017/S0140525X13000447}

Sidney R. Lehky, ${ }^{a}$ Anne B. Sereno, ${ }^{b}$ and Margaret E. Sereno ${ }^{c}$ ${ }^{a}$ Computational Neurobiology Laboratory, The Salk Institute, La Jolla, CA 92037; ' Department of Neurobiology and Anatomy, University of Texas Health
Science Center, Houston, TX 77030; ${ }^{\circ}$ Department of Psychology, University of Oregon, Eugene, OR 94703.

sidney@salk.edu anne.b.sereno@uth.tmc.edu

msereno@uoregon.edu

http://www.snl.salk.edu/ sidney/

http://nba.uth.tmc.edu/homepage/sereno/sereno/index.htm

http://psychweb.uoregon.edu/ serenolab/

Abstract: The target article does not consider neural data on primate spatial representations, which we suggest provide grounds for believing that navigational space may be three-dimensional rather than quasi-twodimensional. Furthermore, we question the authors' interpretation of rat neurophysiological data as indicating that the vertical dimension may be encoded in a neural structure separate from the two horizontal dimensions.

The neurophysiological data presented by Jeffery et al. in the target article in support of the view that spatial representations for navigation by surface-travelling mammals are quasi-planar come from just one animal: the rat. There is no consideration of neural data from primates. We suggest here that when primate neural data are examined, it raises the possibility that primates have three-dimensional volumetric spatial representations for navigation, not quasi-planar ones. Furthermore, we question the authors' interpretation of rat physiological data as suggesting that the encoding of the vertical dimension occurs in a different neural structure from the horizontal dimensions, "in an as yet undiscovered region" (sect. 5.1, para. 5).

One indication of possible differences in spatial representations between rats and primates comes from comparing allocentric responses in the hippocampus. Rat hippocampal place cells fire only when a rat is located at a particular place. On the other hand, macaque monkeys also have hippocampal cells that respond when a monkey is merely looking from a distance at a particular spot, whose location has both horizontal and vertical components (Georges-François et al. 1999; Rolls 1999).

Although neurophysiological studies of navigation in rats have focused on the hippocampus, neurophysiological and functional magnetic resonance imaging (fMRI) studies in macaque monkeys and humans have highlighted other structures as also important during navigation. Among them are the posterior parietal cortex, posterior parahippocampus, and retrosplenial cortex (RSC), together with the nearby posterior cingulate cortex (PCC) (Epstein 2008; Kravitz et al. 2011). RSC and PCC appear to be part of a network that transmits spatial information for navigation from the posterior parietal cortex to medial temporal lobe structures, in particular the parahippocampus and hippocampus.

In addition to being the ultimate source of spatial information for navigation, the posterior parietal cortex is also involved in spatial representations for the control of action (which may be distinct from spatial representations for object recognition and memory [Sereno \& Lehky 2011]; see also earlier work by Goodale \& Milner 1992). Control of action includes control of 3D eye movements and 3D visually guided reaching and grasping by the arm and hand (Blohm et al. 2009; Breveglieri et al. 2012; Hadjidimitrakis et al. 2012). Spatial representations for the control of action in primates operate in a $3 \mathrm{D}$ volumetric space, and not a quasi-2D multiplanar space. Furthermore, recent physiological studies in monkeys of populations of posterior parietal cells (Sereno \& Lehky 2011) show evidence for a $3 \mathrm{D}$ representation of space in primates even when simply fixating.

As the posterior parietal cortex in primates appears to be a source of spatial information both for control of action and for navigation, it seems a reasonable conjecture that known parietal 3D spatial representations for control of action could also be used for navigation. While the dimensionality of space representation for navigation in primates is an important topic that has not been well studied, there are physiological reasons to believe that it may be three-dimensional. 
It is also not clear that the representation of space in the rat is quasi-two-dimensional, as Jeffery et al. claim, in the sense that information about the vertical dimension is processed by different neural circuitry. The authors cite physiological evidence of neural activity in navigation circuits that is modulated by the vertical dimension; for example, elongated vertical (relative to horizontal) place-cell and grid-cell receptive fields for vertically arranged spatial layouts. It doesn't follow from those response anisotropies that medial temporal lobe structures are not encoding the vertical dimension. Computational studies, for example, might establish that observed properties of rat grid and place cells are sufficient to account for behavioral abilities within the vertical dimension without postulating other unknown neural centers for vertical spatial representations. Indeed, there is a debate within the theoretical literature about whether narrowly tuned or coarsely tuned cells provide better representations within a population (Lehky \& Sereno 2011; Pouget et al. 1999; Zhang \& Sejnowski 1999). As the authors themselves state, "much remains to be determined about vertical processing in the navigation system" (sect. 4.1, para. 10). Therefore, the conclusion of the target article that space for navigation is quasi-two-dimensional for all surface-travelling mammals may be premature.

\section{Development of human spatial cognition in a three-dimensional world}

\section{doi:10.1017/S0140525X13000459}

Kate A. Longstaffe, Bruce M. Hood, and lain D. Gilchrist School of Experimental Psychology, University of Bristol, Bristol BS8 1TU United Kingdom.

Kate.longstaffe@Bristol.ac.uk Bruce.Hood@Bristol.ac.uk lain.gilchrist@Bristol.ac.uk http://www.bristol.ac.uk/expsych/people/kate-a-longstaffe/index.html http://eis.bris.ac.uk/ psidg/homepage.html

http://www.bristol.ac.uk/expsych/people/bruce-m-hood/index.html

Abstract: Jeffery et al. accurately identify the importance of developing an understanding of spatial reference frames in a three-dimensional world. We examine human spatial cognition via a unique paradigm that investigates the role of saliency and adjusting reference frames. This includes work with adults, typically developing children, and children who develop non-typically (e.g., those with autism).

Jeffery et al.'s target article explores behavioural and neurobiological studies from many species and draws attention to the gaps in the literature on human three-dimensional navigation. Our own work addresses this gap by using a novel paradigm to examine human searching behaviour in large-scale space. We have used this setup in a series of adult and developmental studies (Pellicano et al. 2011; Smith et al. 2005; 2008; 2010).

Our large-scale search laboratory contains an array of green light-emitting diode (LED) light switches embedded in the floor. Participants press illuminated switches to discover targets that change colour when pressed. Potential targets can be manipulated by colour, saliency, and probability of distribution. Our paradigm preserves the experimental rigor of classic visual search, by recording button presses to millisecond accuracy, while scaling up to large-scale search in which the human participant has to move through the search array. This provides a first step towards investigating the processes involved in human navigation, which addresses a concern that has been raised regarding the validity of modelling human search solely via two-dimensional computer monitor-based search (Dalrymple \& Kingstone 2010; Foulsham et al. 2011; Foulsham \& Kingstone 2012; Ingram \& Wolpert 2011).

Work comparing visually guided versus hidden target conditions revealed increased search latencies when the target is not visually available (Smith et al. 2008). In both visually and non-visually guided conditions there were fewer revisits to previously explored locations than are present in classic computer screen visual search studies, emphasizing a key distinction between visual search and large-scale search (Smith et al. 2008).

Jeffery et al. also highlight a gap in human developmental work on navigation and spatial cognition, which is crucial to building an understanding of how humans develop complex spatial representations. Research in our paradigm has investigated the role of short-term memory in preventing revisits to previously inspected locations (Smith et al. 2005). Children made significantly more revisits to previously examined search locations when performing a more effortful search with the non-dominant hand, suggesting that when the task is more effortful, individuals are less able to optimize search. In addition, although children's general fluid intelligence was unrelated to search time, there was a significant relationship between search latency and visuospatial short-term memory (measured via Corsi blocks). These data highlight the role of spatial working memory in the development of efficient exploration of large-scale space (Smith et al. 2005).

Further work focused on the learning of likely target locations (Smith et al. 2010). Here, targets were located on one side of the room in $80 \%$ of trials. Participants were able to learn likely targets when both the start location and search locations were fixed throughout the task. However, when room-based and reference frames were disrupted, there was no evidence of learning, suggesting that encoding of likely locations depends on a combination of egocentric and allocentric cues (Smith et al. 2010).

This same paradigm has been used in individuals with autism, following the suggestion by Baron-Cohen (e.g., Baron-Cohen 2008) that the exceptional visual search skills of children with autism are due to an increased aptitude at systemizing spatial cognition. However, children with autism were less efficient, less systematic, less optimal, and overall less likely to follow probabilistic cues than ability-matched typical children. This provides further evidence that visual search and large-scale search do not always share the same cognitive mechanisms (Pellicano et al. 2011).

Recent work has investigated the role of saliency in building spatial representations. In work with adult participants, the perceptual salience of search locations was manipulated by having some locations flashing and some static, a paradigm adapted from established visual search literature (Theeuwes 1994; Yantis \& Jonides 1984). Adults were more likely to search at flashing locations, even when explicitly informed that the target was equally likely to be at any location (Longstaffe et al. 2012). We propose that attention was captured by perceptual salience, leading to an automatic bias to explore these targets. When this work was extended to a population of typically developing children, they were also more likely to search at flashing locations, and the magnitude of this effect did not vary with age. However, there was a strong developmental trend in the number of times children revisited previously examined locations. The developmental trajectory for ability to remember previously visited locations and limit revisits shows a development in spatial working memory occurring separately from perceptual inhibition. This suggests individual executive sub-processes may play different roles during search, with different developmental trajectories. Further work in this paradigm examines the effect of mediating working memory load on search behaviour. This investigation of working memory during search is crucial for a transition into real-world tasks, such as searching for lost keys or a lost car in a parking lot.

The review by Jeffery et al. commendably addresses the scope of research across many species and paradigms; here we present work in a novel paradigm that expands our understanding of human adult and child populations in the three-dimensional world. Understanding how individuals incorporate reference frames and employ cognitive mechanisms such as memory and attention is key to modelling human navigation. 


\section{Has a fully three-dimensional space map never evolved in any species? A comparative imperative for studies of spatial cognition}

\section{doi:10.1017/S0140525X13000460}

\section{Cynthia F. Moss}

Department of Psychology, Institute for Systems Research, University of Maryland, College Park, MD 20742.

cynthia.moss@gmail.com

http://www.isr.umd.edu/faculty/gateways/moss.htm

Abstract: I propose that it is premature to assert that a fully threedimensional map has never evolved in any species, as data are lacking to show that space coding in all animals is the same. Instead, I hypothesize that three-dimensional representation is tied to an animal's mode of locomotion through space. Testing this hypothesis requires a large body of comparative data.

The target article by Jeffery et al. reviews the literature on threedimensional spatial navigation and highlights important considerations for interpreting both behavioral and neurobiological data on this topic. In their review, they lay the foundation for the hypothesis that three-dimensional spatial navigation is "bicoded" that is, based on different encoding mechanisms for horizontal and vertical space. Furthermore, they argue that a fully threedimensional map has never evolved in any species.

The "bicoded" model is based on studies of animals that move along surfaces to navigate, which constrains the design of experiments and the data used to build theory. For a complete theory of three-dimensional spatial navigation, a wider range of animals must be studied. In particular, data from animals that freely navigate three-dimensional volumetric space will help determine whether a single model can account for three-dimensional spatial navigation in terrestrial, aerial, and aquatic species. Counter to the model proposed by the authors of the target article, I hypothesize that a fully three-dimensional volumetric map has evolved in species that are not limited to navigating along surfaces. More broadly, I hypothesize that three-dimensional spatial representation depends upon an animal's mode of locomotion and the navigational tasks it encounters in the natural environment.

Another point I would like to make is that models of threedimensional spatial navigation must be grounded in behavioral and neurobiological studies of animals engaged in biologically relevant and natural tasks. For example, rodents have conveniently served as animal models for a wide range of studies, including spatial navigation. Many rodent maze studies are, however, highly artificial, requiring that animals perform tasks they are unlikely to encounter in a natural setting, and in spaces far more restricted than they would navigate in the wild. The study of inbred rodents that have not navigated the natural environment for many generations raises additional concerns.

Research traditions have limited the study of spatial navigation, and advances in the field require a comparative approach. The importance of choosing the right animals for the questions under study, first articulated by Nobel Laureate August Krogh (1929), is widely recognized in the field of Neuroethology, but is less influential in the broad field of Systems Neuroscience. It is important that the spatial navigation research community interested in problems of three-dimensional spatial navigation turn to the study of animals that have evolved to solve this problem.

Echolocating bats present an excellent model system to pursue questions about three-dimensional spatial navigation. Bats belong to the order Chiroptera, many species of which use biological sonar systems to represent the spatial location of targets and obstacles. In turn, this spatial information is used to build a cognitive map that can guide navigation in the absence of sensory cues. Anecdotal reports and laboratory studies of echolocating bats provide evidence that bats rely strongly on spatial memory (Griffin 1958; Jensen et al. 2005), and field studies show that bats use memory on many different spatial scales (Schnitzler et al. 2003; Tsoar et al. 2011). Importantly, bats use an absolute-space-based (allocentric) navigational strategy that is hippocampus-dependent, and place cells have been identified and characterized in the bat hippocampus (Ulanovsky \& Moss 2007; Yartsev \& Ulanovsky 2013). Furthermore, grid cells have been recently described in the medial entorhinal cortex (MEC) of the Egyptian fruit bat (Yartsev et al. 2011). Therefore, echolocating bats are particularly well suited for researchers to use in behavioral, neurobiological, and computational studies of three-dimensional spatial navigation.

Neural recording data from the MEC and hippocampus of bats have raised questions about the generality of the rodent model in spatial representation. Although neural recordings from awake, behaving rats and bats show that neurons in the hippocampus and MEC represent two-dimensional space in a similar way (Ulanovsky \& Moss 2007; Yartsev et al. 2011), there is one noteworthy difference, namely an absence of continuous theta oscillations in hippocampal recordings from the big brown bat (Ulanovsky \& Moss 2007) and the MEC and hippocampus in the Egyptian fruit bat (Yartsev et al. 2011; Yartsev \& Ulanovsky 2013). Whole cell patch clamp studies of the MEC of the big brown also demonstrate differences in subthreshold membrane potential resonance between bats and rats (Heys et al. 2013). These reported species differences challenge a fundamental assumption of the oscillatory interference model of spatial coding (e.g., Burgess et al. 2007; Hasselmo et al. 2007). By extension, comparative data could raise questions about the generality of three-dimensional spatial coding mechanisms in the brains of animals that have evolved to navigate along surfaces, compared with those that move freely through three-dimensional volumetric spaces, such as air and water.

In summary, I propose that it is premature to assert that a fully three-dimensional map has never evolved in any species, as empirical data are lacking to support the notion that three-dimensional space coding in all animals is the same. Recent findings from Yartsev \& Ulanovsky (2013), demonstrating three-dimensional space representation in the hippocampus of the freeflying Egyptian Fruit bat, are consistent with the hypothesis that space representation is tied to an animal's mode of locomotion. To fully test this hypothesis requires a large body of comparative data.

\section{ACKNOWLEDGMENT}

Supported by ONR Grant (N00014-12-1-0339), Three-dimensional Spatial Navigation, to Cynthia F. Moss and Timothy Horiuchi.

\section{Making a stronger case for comparative research to investigate the behavioral and neurological bases of three-dimensional navigation}

\author{
doi:10.1017/S0140525X13000472
}

\section{Daniele Nardi a and Verner P. Bingman ${ }^{\mathrm{b}}$ \\ a Department of Psychology, Sapienza Università di Roma, 00185 Rome, Italy; ${ }^{\mathrm{b}}$ Department of Psychology and J.P. Scott Center for Neuroscience, Mind, and Behavior, Bowling Green State University, Bowling Green, OH 43403. d.nardi@uniroma1.it vbingma@bgsu.edu}

Abstract: The rich diversity of avian natural history provides exciting possibilities for comparative research aimed at understanding threedimensional navigation. We propose some hypotheses relating differences in natural history to potential behavioral and neurological adaptations possessed by contrasting bird species. This comparative approach may offer unique insights into some of the important questions raised by Jeffery et al. 
Jeffery et al.'s target article is a fascinating synthesis of the relatively scarce but expanding research on three-dimensional navigation, in addition to proposing an engaging hypothesis on the neural representation of vertical space. As recognized by Jeffery et al., birds offer intriguing possibilities as model species for investigating the behavioral and neurological bases of navigation in 3D space. Studies on homing pigeons (Columba livia) suggest that the topography of the landscape is an important source of information for bird navigation. As mentioned by Jeffery et al., sloped terrain provides a global feature and - at least locally - a directional reference frame. The extent to which pigeons encode and use this reference frame, when walking on a slanted floor, is impressive (Nardi \& Bingman 2009a; Nardi et al. 2010). A crucial attribute of slope-and more generally, the vertical dimension-is the involvement of effort. This has been highlighted in rats, which show a strategy preference for horizontal movements relative to vertical movements (Grobéty \& Schenk 1992a; Jovalekic et al. 2011). In pigeons, evidence suggests that effort, modulated by different, energetically demanding upward and downward movements, affects how a sloped environment is represented, or at least used (Nardi et al. 2012). This strengthens the hypothesis that effort could be used as a "contextual" cue, in the sense proposed by Jeffery et al., derived from kinesthetic/proprioceptive information during locomotion on a slope. Importantly, hippocampal lesions in pigeons do not impair the slope-based goal representation (Nardi \& Bingman 2009b), despite having disruptive effects on memory for other spatial cues (e.g., Gagliardo et al. 1999; Vargas et al. 2004). Possibly slope does not engage the hippocampus because it is used more as a source of directional, compass-like information than for determining distances and map-like information. More studies are needed to ascertain the role of effort in the representation of sloped - and volumetricspace, and its neurological underpinnings.

Beyond pigeons, the class Aves, with its rich diversity in natural history, offers exciting opportunities to apply comparative methods for better understanding the representation of 3D space. Although Jeffery et al. acknowledge that evolutionary adaptation (and constraints), reflected in species natural history, may be important in how vertical and horizontal space may be integrated, they offer few explicit hypotheses that can be answered by comparative-based research. As a well-known example of the power of comparative research, the larger hippocampus and superior spatial cognitive abilities of food-storing birds (e.g., Pravosudov \& Clayton 2002) indicate that differential evolutionary pressure can lead to variation in natural history, which co-occurs with variation in brain-behavior organization. We are similarly convinced that interesting differences can also be found with respect to navigating $3 \mathrm{D}$ space at both the relatively easily studied behavioral level and the more difficult to study neurobiological level. Almost all species of birds fly and necessarily inhabit a three-dimensional world, but often differ with respect to their "3D occupancy profile." For example, ground birds, such as some quail species (family Odontophoridae) inhabit a robustly horizontal space, whereas tree creepers (family Certhididae) search for food vertically but move horizontally among different trees, and wallcreepers (family Tichodromidae) live in the substantially vertical world of cliff faces. ${ }^{1}$ How might natural differences in horizontal, vertical, and volumetric occupancy influence the representation of space and its neural encoding in the hippocampal formation or elsewhere in the brain?

In our opinion, the most exciting and novel hypothesis advanced by Jeffery et al. is the notion that horizontal space is more richly represented and is dissociated, at least partially, from the relatively impoverished, contextual representation of vertical space. And this may indeed be true for species such as rats and even humans. We wonder, however, how generally applicable this idea may be. The black-capped chickadee (Poecile atricapillus) is a remarkable species of bird that routinely lives in the complex volumetric space of deciduous trees and will seasonally store and later recover seeds within the chaotic world of intersecting tree branches - a behavior dependent on the integrity of the hippocampal formation (Sherry \& Vaccarino 1989). Chickadees apply their robust, spatial memory ability in a 3D space, which may appear multilayered but, because deciduous tree branches can intersect at almost any angle, would often be better characterized as true volumetric space. (Coniferous trees have a more multilayered quality.) The survival of individuals is very much dependent on "volumetric encoding" of stored food that would likely suffer if only a contextual heuristic would be used to encode vertical location. From a purely adaptationist perspective, if any species - and more generally, any natural history profile-would support the evolution of a unified representation of volumetric space, it would be the black-capped chickadee and other species that store seeds in deciduous trees. As a natural comparative experiment, chickadees could be contrasted with the Clark's nutcracker (Nucifraga columbiana), which also stores seeds and has an extraordinary ability to recall the locations of those seeds (e.g., Kamil et al. 1994). However, whereas chickadees store seeds within the volumetric space of tree limbs, nutcrackers store them in the ground, in a rolling but two-dimensional horizontal space. Would chickadees outperform nutcrackers in a spatial memory task in a sloping, multilayered or volumetric space? Would hippocampal neurons in chickadees be more likely to encode aspects of volumetric space?

We are not advocating that comparative approaches would answer all the important questions raised by Jeffery et al., but we hope we have convinced the reader that comparative research - not necessarily limited to birds - is a powerful experimental strategy that can reveal much about the diversity of three-dimensional spatial representations.

ACKNOWLEDGMENT

We would like to thank David Sherry for providing some helpful advice.

\section{NOTE}

1. We want to acknowledge here that comparative studies have interpretative challenges associated with them and that the best comparisons are among taxonomically closely related species (Harvey \& Pagel 1991). As such, quails are probably not a good comparison group, and we use them only as a convenience to make our point.

\section{Which animal model for understanding human navigation in a three-dimensional world?}

\section{doi:10.1017/S0140525X13000484}

\section{Guy A. Orban}

Department of Neuroscience, Division of Neurophysiology, Parma University, 43100 Parma, Italy.

guy.orban@med.kuleuven.be

Abstract: Single-cell studies of monkey posterior parietal cortex (PPC) have revealed the extensive neuronal representations of threedimensional subject motion and three-dimensional layout of the environment. I propose that navigational planning integrates this PPC information, including gravity signals, with horizontal-plane based information provided by the hippocampal formation, modified in primates by expansion of the ventral stream.

Jeffery et al. ignore half of navigation, namely navigation in egocentric frameworks. Many of the mechanisms of this type of navigation - as important as allocentric navigation, as walking along a cliff demonstrates - have been investigated in primates, but are conspicuously absent from the present review. Yet, from an evolutionary and genetic standpoint, we humans are obviously much closer to apes and monkeys than to rodents. Monkeys typically live and climb in trees and, not surprisingly, possess a threedimensional (3D) space representation in their posterior parietal 
cortex (PPC) (Andersen \& Buneo 2002). The PPC is dramatically expanded in primates compared to rodents, in which primary cortices V1 and S1 lie very close, leaving little room for the extrastriate or posterior parietal cortex (Whitlock et al. 2008). Single-cell studies in monkeys have revealed neurons selective for optic flow components in several parietal regions: middle superior temporal dorsal (MSTd: Lagae et al. 1994; Tanaka et al. 1986), ventral intraparietal (VIP: Schaafsma \& Duysens 1996), and medial PPC (Squatrito et al. 2001). Human imaging studies suggest that additional regions, such as V6, might be involved (Wall \& Smith 2008). DeAngelis, Angelaki, and coworkers have compared visual and vestibular selectivities of neurons in MSTd, VIP, and visual posterior sylvian (VPS) for rotation and translation in three dimensions (see Chen et al. 2010; 2011a; 2011b; Takahashi et al. 2007). Whereas MSTd and VIP neurons can support the perception of heading, neurons in VIP and VPS support those of selfrotation. In these areas, all directions in space are represented with no clustering of preferences close to the horizontal plane, as would be expected if the vertical direction were poorly represented. This is unsurprising as in primates equipped with binocular vision the $z$-axis (depth) is unique, not the vertical axis, which unlike depth is represented on the retina. Indeed, particularly in VIP and to a lesser degree in MSTd, the depth axis was under-represented among preferred directions. Preferred directions and tuning widths of MSTd neurons, however, were nonuniformly distributed along the horizontal meridian, explaining the decrease in behavioral sensitivity to self-motion direction with increasing eccentricity in humans and monkeys ( $\mathrm{Gu}$ et al. 2010).

In their quest to demonstrate that humans mainly use a horizontal reference frame for navigation, Jeffery et al. quote psychophysical studies of 3D slope perception and the misperception of vertical slopes they reveal. Contrary to the authors' claims, this misperception is part of a larger problem, depth reconstruction (see above), which is complicated by the dependence of distance scaling on the derivation order of disparity. Indeed, human subjects have no precise representation of metric 3D structure, whether using stereo or monocular cues (Todd \& Norman 2003). In no way can these misperceptions be taken as evidence that neuronal mechanisms for estimating 3D slopes do not exist. In fact, such neurons have been documented in caudal intraparietal (CIP), part of the PPC (Taira et al. 2000), and also in the inferotemporal cortex (Liu et al. 2004) - thus, in both the dorsal and ventral visual systems. Similarly, selectivity for second-order disparity gradients has also been documented (see Orban 2011 for review). Some of the cortical areas in which these higher-order neurons have been recorded may not even exist in rodents, again underscoring the importance of using adequate models for the human brain. Together, these results indicate that the primate PPC includes representations in three dimensions of subject motion and of the layout of the environment, two critical pieces of information for navigating through 3D space.

The starting point of the target article is the importance of the cognitive map in the hippocampus for spatial navigation. Even if grid cells have recently been identified in monkey entorhinal cortex (Killian et al. 2012), the hippocampal system of primates bears only a vague resemblance to that of rodents. Relatively small numbers of human hippocampal neurons are place cells, and these occur in several brain regions (Jacobs et al. 2010). This is not surprising, as the hippocampus receives major projections from the ventral stream (Kravitz et al. 2013), which in primates processes information about objects at least as much as scenes, and even information concerning conspecifics. Indeed, considerable evidence indicates that the human hippocampal formation processes not only spatial, but also non-spatial and even non-visual information (Liang et al. 2013). This species difference may be even further underestimated, as the human brain evolved to accommodate bipedalism, and thus navigation in open spaces, but such navigation has so far been little investigated for technical reasons. On the other hand, navigation supposes a transformation of coordinate information from spatial maps into body-centered representations, a function likely to be subserved by the PPC, which is connected to the hippocampal formation via the retrospenial cortex in rodents (Whitlock et al. 2008). A similar route links the primate precuneus and adjacent dorsal superior parietal lobule with the hippocampal system (Kravitz et al. 2011). The involvement of these medio-dorsal PPC regions in the control and planning of navigation is supported by their activation during observation of climbing and locomotion in humans (Abdollahi et al. 2012). Hence, I propose a much more balanced view, whereby spatial navigation is both allocentric and egocentric and is controlled by both the hippocampal formation (fed by the qualitative scene analysis in parahippocampal gyrus where gravity is not incorporated) and the metric space representation in the PPC (in which gravity is incorporated). These two streams of information are integrated in the dorso-medial PPC devoted to planning locomotor body movements. A recent imaging study indicates that this scheme, clearly different from the bicoded scheme of Jeffery et al., applies to humans (Indovina et al. 2013). To what extent it may also apply to rodents is unclear but, intriguingly, one of the subregions of the rodent PPC (the anteromedial visual area; Torrealba \& Valdes 2008) may be a precursor of primate V6 and supply optic flow information.

In conclusion, including nonhuman primates among the animal models is essential for understanding the human brain, even for navigation and spatial memory, avoiding errors induced by jumping from rodents to humans as these authors have done.

\section{The study of blindness and technology can reveal the mechanisms of three-dimensional navigation}

\author{
doi:10.1017/S0140525X13000496
}

\section{Achille Pasqualotto ${ }^{\mathrm{a}}$ and Michael J. Proul $\mathrm{x}^{\mathrm{b}}$ \\ ${ }^{a}$ Biological and Experimental Psychology Group, School of Biological and Chemical Sciences, Queen Mary University of London, London E1 4NS, United Kingdom; ${ }^{\mathrm{b}}$ Department of Psychology, University of Bath, Bath BA2 7AY, United Kingdom. \\ pasqualotto@qmul.ac.uk m.j.proulx@bath.ac.uk http://people.bath.ac.uk/mjp51/}

Abstract: Jeffery et al. suggest that three-dimensional environments are not represented according to their volumetric properties, but in a quasiplanar fashion. Here we take into consideration the role of visual experience and the use of technology for spatial learning to better understand the nature of the preference of horizontal over vertical spatial representation.

The article by Jeffery et al. reviews the literature on spatial cognition relative to three-dimensional environments, which is an area vastly less investigated than are two-dimensional (or planar) environments. The authors have concluded that three-dimensional spaces are represented in a quasi-planar fashion. Here we add to the discussion by examining the role played by developmental vision on the structure and functioning of brain areas involved in spatial cognition, and the results of studies investigating the role of spatial learning on three-dimensional space representation.

The role of developmental vision in spatial representation has been reported by numerous studies on humans (Pasqualotto et al. 2013; Postma et al. 2007; Röder et al. 2007; Vecchi et al. 2004; see Pasqualotto \& Proulx [2012] for a review) and animals (Buhot et al. 2001; Hyvärinen et al. 1981; Paz-Villagrán et al. 2002). Yet, the role played by vision on the establishment of the quasi-planar representation of three-dimensional spaces has not been investigated. This represents a broad avenue for future studies. Due to the crucial role played by vision in spatial cognition 
(e.g., Frankenstein et al. 2012) and vertical spatial perception (Ooi et al. 2001; Sinai et al. 1998), it is conceivable that visual experience has an effect on both horizontal and vertical spatial representation, and, therefore, the results discussed by Jeffery et al. may be driven by experience with the visual world. In other words, blind individuals, especially if congenitally blind, may not exhibit quasiplanar spatial representation of three-dimensional environments. This hypothesis may be well supported by studies reporting the role of blindness in the functioning of hippocampal cells in rodents (Hill \& Best 1981; Larsen et al. 2009; Paz-Villagrán et al. 2002; Poucet et al. 2000) and on hippocampal structure and activation in humans (Chebat et al. 2007; Deutschländer et al. 2009; Kupers et al. 2010; Leporé et al. 2009).

The study by Passini and Proulx (1988) represents a rare investigation on the spatial representation of a three-dimensional environment by congenitally blind and sighted participants. They found that overall, blind participants took more decisions at crucial points of the environment than did sighted ones. These points included intersections, stairs, open spaces, etc. Crucially, vertical structures of the environment did not receive more attention than horizontal ones.

In a recent article, Thibault et al. (2013) investigated the effect of spatial learning on memory of a virtual three-dimensional building. The same building was learned either through "planar" or "columnar" exploration. It was followed by a spatial memory task where participants re-experienced a segment of the exploratory route. The results showed that the group of participants who underwent planar exploration had superior spatial memory performance. Yet, Thibault et al. also found that for both groups, the spatial memory task was better performed when participants re-experienced the same segments as during the exploratory phase (i.e., congruency effect). This suggests that, according to the learning type, participants could store the three-dimensional building in quasi-planar and quasi-columnar fashions.

These results suggest that quasi-planar spatial representation may be the result of quasi-planar spatial exploration. In other words, by using virtual reality (Lahav \& Mioduser 2008; Péruch et al. 1995) or sensory substitution devices (Kupers et al. 2010; Proulx et al. 2008; 2012), three-dimensional environments could be explored and stored according to any fashion (i.e., planar or columnar). These devices would overcome the energetic cost associated with vertical exploration (Butler et al. 1993; Hölscher et al. 2006). Finally, the ability of the brain to learn and carry out spatial memory tasks in non-planar fashion is supported by studies in "gravity-less" three-dimensional virtual mazes (Vidal et al. 2003; 2004). Therefore, we advocate that future research should address the role played by visual experience and spatial learning on spatial representation of three-dimensional environments.

\section{Augmented topological maps for three- dimensional navigation}

\section{doi:10.1017/S0140525X13000502}

\section{Herbert Peremans and Dieter Vanderelst \\ Active Perception Lab, FTEW-MTT, Universiteit Antwerpen, 2000 Antwerpen, Belgium. \\ herbert.peremans@ua.ac.be Dieter.Vanderelst@ua.ac.be \\ http://www.ua.ac.be/main.aspx?c=herbert.peremans \\ http://bitsofbats.weebly.com/}

\begin{abstract}
We describe an augmented topological map as an alternative for the proposed bicoded map. Inverting causality, the special nature of the vertical dimension is then no longer fixed a priori and the cause of specific navigation behavior, but a consequence of the combination of the specific geometry of the experimental environment and the motor capabilities of the experimental animals.
\end{abstract}

Based on a review of neurophysiological and behavioural evidence regarding the encoding of three-dimensional space in biological agents, Jeffery et al. argue for a bicoded map in which height information is available and used but not fully integrated with information from the horizontal dimensions in the cognitive map. We propose an alternative hypothesis, namely an augmented topological map with "semi-metric" properties that under certain circumstances will give rise to a representation which appears to be bicoded but is based on the same principles of spatial encoding operating in all three dimensions.

As an alternative to a metric representation, roboticists (Kuipers et al. 2000; Wyeth \& Milford 2009) have found that a topological representation suffices for robots to map and navigate planar environments. In these experiments, a graph representation, with nodes corresponding to distinct places and edges to specific motor programs, enables the robot to follow a path from one distinct place to another. To also allow the capability of executing shortcuts, this graph representation is augmented with metric information. Sensor-based estimates of travelled distance and travel direction associated with the edges allow assignment of metric coordinates to the nodes in the graph. These estimates will initially be unreliable. However, a mechanism as described by Wyeth and Milford (2009) corrects the metric coordinate estimates for the graph nodes when the agent returns to a previously visited distinct place. As a consequence, this representation converges to a "semi-metric" map. This differs from a real metric map in that the reliability of the metric information associated with the nodes varies across the graph. For sparsely connected subgraphs, for example less-travelled parts of the environment, the metric information can be unreliable. For highly interconnected subgraphs - for example, well-explored parts of the environment - the metric information can be used for optimizing paths: for instance, in calculating shortcuts.

We propose that such a scheme can be extended to three dimensions: the nodes represent three-dimensional positions, and the edges the three-dimensional motor programs that move the agent from one position (i.e., sensorially distinct place) to another. If the environment allows multiple three-dimensional paths from one place to another, a "semi-metric" three-dimensional map would result. Elsewhere we have described a mechanical analogue (Veelaert \& Peremans 1999) to model this process, in which we map the nodes in the graph onto spherical joints and the edges onto elastic rods. To represent both the uncertainty about the actual distance travelled and the direction of travel between the distinct places represented by the nodes, both the lengths of the rods and their orientations can vary (see Fig. 1). The rigidity of the resulting mechanical construction tells us whether all nodes are at well-defined positions relative to one another. Whenever particular subparts of the mechanical construction can still move relative to one another, this indicates that the corresponding parts of space are not well defined with respect to each other. However, if the subparts of the mechanical construction are rigid, relative positions within those parts of space can be fully defined.

Multilayer environments modeled with this representation result in a discrete set of highly interconnected subgraphs, one for each layer, connected together by a small number of isolated links. The isolated links correspond to the few paths that can be followed to go from one layer to another; for example, a staircase in a building. As the subgraphs are connected by a few links only, they can be metrically related to each other but the uncertainty on this relationship is higher than that between distinct places at the same layer. The metric representations of the different layers are only partially registered. In terms of our mechanical analogue, the constructions representing the individual layers are rigid, but these rigid substructures are linked to one another through a few links only, allowing them to move relative to each other as illustrated in Figure 1.

From the mechanical analogue, it is clear that the extent to which the augmented topological representation of space could 
(a)

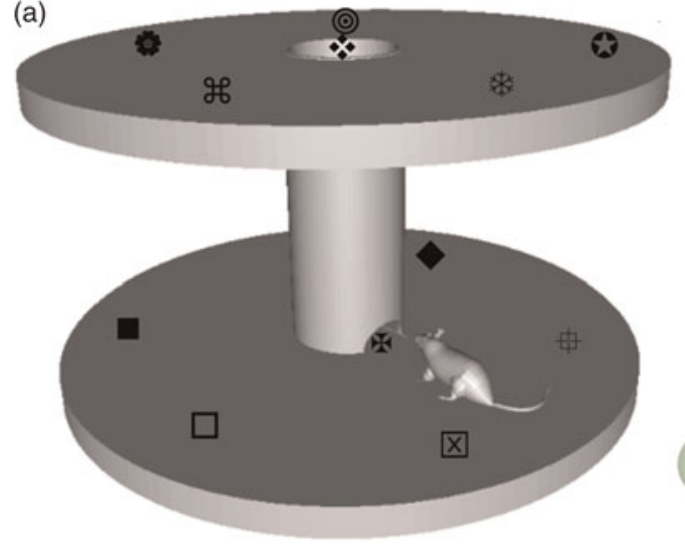

(b)

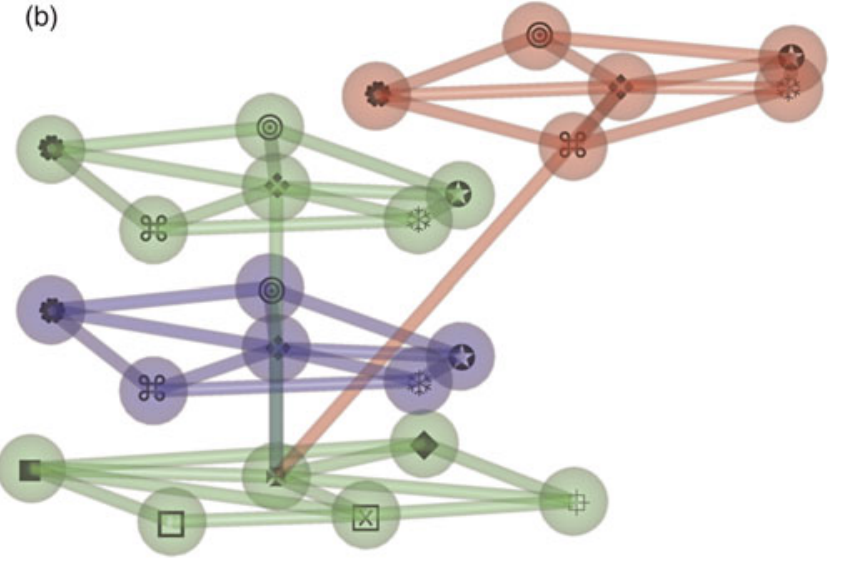

Figure 1 (Peremans \& Vanderelst). (a) Two-layered environment with marked distinct places; (b) three hypothetical representations of $(\mathrm{a})$, where green $=$ accurate travelled distance and orientation estimates, blue $=$ inaccurate travelled distance estimate, and red $=$ inaccurate travelled distance and orientation estimates.

act as a metric representation - that is, give rise to a rigid mechanical construction - depends on the interconnectivity of the nodes in the graph. One factor that determines this interconnectivity is whether the environment provides many opportunities for following different closed-loop paths that return to already-visited distinct places. Furthermore, given an environment providing many such opportunities, whether a highly interconnected graph would actually be constructed also depends on the movement patterns of the mapping agent. For example, the rats in the vertical plane maze seem to prefer movement patterns that extensively explore horizontal layers, only sparsely interspersed with short movements up or down the vertical dimension. Such a movement pattern gives rise to a set of highly interconnected subgraphs linked together by a few isolated links, similar to that of a multilayer environment. Hence, to really test whether the augmented topological representation (and the mechanism by which a three-dimensional "semi-metric" representation arises from it) corresponds with the actual cognitive map as implemented in mammalian brains, the experimental agents should be able to treat all spatial dimensions equivalently.

We propose that bats are a promising animal model for such a study, as they live in a true three-dimensional environment and have the motor capabilities to follow arbitrary three-dimensional paths through this environment. In particular, the spatial memory and orientation of nectar-feeding bats using both visual and echo-acoustic environment sensing have already been investigated in a series of studies (Thiele \& Winter 2005; Winter et al. 2004). Interestingly, Winter and Stich (2005) note that the hippocampus of nectar-feeding glossophagine bats is 50-100\% larger in size than that of insectivorous members of the same family (Phyllostomidae). Although the reported results do not show evidence that these animals treat vertical and horizontal dimensions differently, more specific experiments are necessary, as the ones described were not aimed at finding out the nature of the bats' cognitive map.

\section{Navigation bicoded as functions of $x-y$ and time?}

\section{doi:10.1017/S0140525X13000514}

James G. Phillips and Rowan P. Ogeil

School of Psychology and Psychiatry, Monash University, Clayton, VIC 3800 , Australia.

Jim.Phillips@monash.edu

Rowan.Ogeil@monash.edu
Abstract: Evidence from egocentric space is cited to support bicoding of navigation in three-dimensional space. Horizontal distances and space are processed differently from the vertical. Indeed, effector systems are compatible in horizontal space, but potentially incompatible (or chaotic) during transitions to vertical motion. Navigation involves changes in coordinates, and animal models of navigation indicate that time has an important role.

Jeffery et al. provide evidence that horizontal and vertical coordinates are processed by different systems, to the effect that navigation in a three-dimensional environment requires two codes. The authors summarise evidence from allocentric navigation and single cell recording to support their position. Although we are more familiar with egocentric coordinate systems, we agree with Jeffery et al. that bicoding is likely, and note evidence from perceptual illusions, neurological conditions, studies of stimulusresponse compatibility, and biomechanical constraints further supporting this.

Perceptual illusions. Distinctly different codes responsible for horizontal and vertical distances are indicated by perceptual illusions. In the horizontal/vertical illusion, two lines of equal length form an upside-down T. Although of equal length, the vertical line nevertheless appears longer perceptually (Avery \& Day 1969). Such observations imply that horizontal and vertical distances are processed differently, and this is reinforced by the differential vulnerabilities of horizontal and vertical coordinate systems to neurological disorders.

Neurological disorders. Cerebro-vascular accidents (strokes) affecting the right hemisphere, particularly the parietal lobe, lead to hemineglect (Mattingley et al. 1992). Hemineglect is a tendency to ignore the left side of space that occurs independently of any deficits in the visual field. These strokes affect the perception of space and the willingness to search and explore the left side of space. Although hemineglect is a relatively common neurological syndrome, a comparable tendency to ignore the lower or upper zones of space is quite rare, and the underlying pathophysiology is not well understood (Shelton et al. 1990), suggesting that different systems are responsible for horizontal and vertical coordinates, and this is reinforced by a consideration of effector systems.

Stimulus-response compatibility. The transition from a horizontal plane to a three-dimensional coordinate system as occurs in three-dimensional interfaces (Phillips et al. 2005), poses potential sources of incompatibility between stimulus and response (Worringham \& Beringer 1989). For instance, in human-computer interfaces, leftwards joystick or leftwards mouse motions both indicate left cursor motion, and remain so in three dimensions. Nevertheless, the use of motions to code for up can be arbitrary, with forward mouse movements coding for upwards cursor 
motion on computer screens and forward joystick motion coding for downwards cursor movements. Indeed, this arbitrariness is also seen within animals, with locomotor responses operating in opposition to trunk/body responses, such that downward propulsive movements code for up (flying, swimming) or head-down movements code for down (flying, swimming).

Biomechanical constraints. Indeed, navigation is necessarily a corollary of activity and motion, and there are biomechanical constraints that argue for motion to be primarily coded in the horizontal plane. Motion occurs within the context of a constant gravitational field that has implications for movement (Phillips \& Ogeil 2010). For most mammals, locomotion is roughly analogous to the oscillations of pendulums suspended vertically from hip or shoulder joints. Such motions are mathematically predictable. However, there are potential sources of problems if motions incorporate additional oscillators to create motion in the third dimension. If motion in the third dimension requires horizontally oriented oscillators, these physical systems have the potential to be chaotic and unpredictable, because the combination of horizontal and vertical oscillators approximates a pendulum ( $x$ oscillator) suspended on a rotating driving arm ( $y$ oscillator). Such systems are potentially chaotic (Baker \& Gollub 1996) in their behaviour, with system characteristics dependent upon speed and the size of the $y$ oscillator. Given a potential unpredictability inherent in motion in three dimensions, it should not be surprising that motion systems are primarily oriented in accordance with gravity and that coordinate systems are oriented perpendicularly to gravity, with other sources of information being more important for navigation than is altitude.

Coordinate systems. Navigation requires motion, with coordinates updated as time and location change. For example, studies of Monarch butterfly navigation during seasonal migration have demonstrated at the cellular level that the circadian system integrates information about navigating space based on time in addition to an insect's position relative to the sun (Reppert 2006). Central to this is a time-compensated sun compass, which likely receives direct input from the circadian system (Zhu et al. 2008), to allow correction of flight direction and coordinates relative to changing light levels across the course of the day. This time-compensated sun compass appears to be genetically determined rather than learned (Reppert 2006). Hence, we feel the second code is more likely to involve a time component than an altitude component.

\section{Vertical and veridical-2.5-dimensional visual and vestibular navigation}

\section{doi:10.1017/S0140525X13000526}

\section{David M. W. Powers}

CSEM Centre for Knowledge and Interaction Technology, Flinders University, Adelaide, SA 5001, Australia.

David.Powers@flinders.edu.au

http://flinders.edu.au/people/David.Powers

\begin{abstract}
Does the psychological and neurological evidence concerning three-dimensional localization and navigation fly in the face of optimality? This commentary brings a computational and robotic engineering perspective to the question of "optimality" and argues that a multicoding manifold model is more efficient in several senses, and is also likely to extend to "volume-travelling" animals, including birds or fish.
\end{abstract}

We think we live in a three-dimensional world, so it is natural to think that our representation of our environment would reflect a three-dimensional structure, and be represented as such in our three-dimensional brain. In fact, we operate in at least a four-dimensional world, with time being a dimension that also needs representation in order to process events, deal with causality, and so forth. The traditional computing model, whether the one-dimensional Turing Machine or the sequential address space of a central processing unit (CPU), is to embed multidimensional structures by arithmetic or referential mappings to linear arrays. Computer circuits and living brains also have a strong, locally two-dimensional structure. In the cortex, the layers of the brain represent both layers of complexity and a mapping of time to depth, with opportunity for increasingly diverse associations to be formed. The cochlear is essentially a one-dimensional representation physically coiled and temporally coded for time and frequency. The retina is essentially a twodimensional representation with quaternary coding for frequency, and again an analog encoding of time and amplitude. The vestibular system is a collection of essentially one-dimensional sensors, as described by Jeffery et al., who note that there would be considerable complexity in producing a three-dimensional analog representation from this.

Although the three-dimensional world nominally has volume "larger than a plane by a power of 3/2" (sect. 2, para. 4), the vertical dimension is in general many orders of magnitude smaller than the distances navigated in the horizontal plane, there is no need to encode it this way, and even "volume-travelling" creatures tend to maintain a preferred orientation in relation to gravity, as Jeffery et al. point out (sect. 5.1, para. 11). Animals also tend to operate in strata, being far from "unconstrained." In fact, there is arguably less constraint for a rat jumping around a three-dimensional maze or a squirrel jumping around an even more complex forest - for example, they can move vertically in a way a bird or a fish cannot. Similarly, the "three planes" of directional information when viewed egocentrically, combined with the vertical dimension "characterized by gravity" (sect. 2 , para. 4), provide a natural two-dimensional manifold which can be topologically expanded locally when there is more information available, as is well known from self-organizing maps (von der Malsburg 1973). The constraints of gravity do not "add" complexity, but rather give rise to both constraints and heuristics that reduce complexity.

The visual system is also constrained by the locus of eyegaze and the characteristic poses with respect to gravity. Here we have twodimensional sensors from which three-dimensional information could be reconstructed, but again the evidence points to a 2.5dimensional model (Marr 1982). But the two-dimensional plane of the retina tends to be near vertical, whereas the two-dimensional plane of locomotion tends to be near horizontal, and it would seem natural that both should track egocentrically when these assumptions are invalid, with discrepancies between the canonical gravitational and egocentric navigational model leading to errors that increase with the deviation.

Jeffery et al. have little to say about navigation, focusing almost exclusively on representation without much comment on aspects of the reviewed experiments that relate to their actual title. When we consider vision and navigation in robotics, whether underwater, aerial, or surface vehicles are used, we tend to use a two-dimensional model coded with far more than just one additional piece of dimensional information. We tend to track and correct individual sensor readings to keep track of our location, based on simple models that predict based on location, velocity, and acceleration, and we also attach information about the terrain, the temperature, the sights and sounds, and potentially also information about how hard or dangerous, or how much energy is being expended. These factors become inputs for both algorithmic and heuristic components of the actual path planning that is the key task in navigation. One additional factor that is important here is the ability to deal with the world at multiple scales, with approximate paths being worked out for a coarse scale before zooming in recursively to plan the coarse stages in detail. Even fully detailed two-dimensional maps lead to impossibly complex calculations of an optimal path, so the solution is to select a reasonable one at a coarse level based on highlevel detail, and then burrow down into the detail when needed. Humans also have difficulty navigating more than two dimensions, 
and even 2.5 dimensions is not in general a good computer interface feature (Cockburn \& McKenzie 2001; 2002).

In terms of Shannon Information, the most frequent events or landmarks or percepts are the least interesting and useful, and Kohonen Self-Organizing Maps (SOM) will tend to allocate area to its inputs in a monotonic increasing but sublinear way $\left(p^{2 / 3}\right.$ rather than $-\log p$ ) for a gain in efficiency of representation, whilst the Zipfian Principle of Least Effort will ensure encoding so that the frequent data can be dealt with faster, and sparse representation principles will lead us to store events that occur rather than placeholders for events that never occur or voxels that are seldom occupied, as would be the case for a four-dimensional model with a cell for every time and place. In fact, the antiShannon allocation of memory in a SOM means there is more real estate available for the subtle details that correspond to the next level of detail in our robotic model. For the squirrel this might be brain space for the vertical dimensions and horizontal layers of a forest, and, more importantly still, the nutritional and territorial dimensions. The latter is an important fifth dimension for the squirrel, marked by its own pheromones and those of its potential mates and potential predators.

Jeffery et al. argue for a 2.5-dimensional model, but allow that this could be a manifold with an egocentric normal rather than a gravimetric plane. They seem to prefer a mosaic model, which is reminiscent of many robotic and vision models where extra details are linked in. The manifold model would in many ways seem to fit better with current ideas of brain processing and self-organization, although a distributed mosaic of contextual associations with multiple sensory-motor areas would be a highly plausible model for survival navigation.

\section{Foreshortening affects both uphill and downhill slope perception at far distances}

\section{doi:10.1017/S0140525X13000538}

\section{Helen E. Ross}

Department of Psychology, University of Stirling, Stirling FK9 4LA, Scotland, United Kingdom.

h.e.ross@stir.ac.uk

www.psychology.stir.ac.uk/staff/staff-profiles/honorary-staff/helen-ross

Abstract: Perceived slope varies with the viewing distance, and is consistent with the effects of foreshortening. Distant viewing makes uphill slopes appear steeper and downhill slopes flatter than does near viewing. These effects are obvious to skiers and climbers in mountainous country. They have also been measured in outdoor experiments with controlled viewing distances. There are many other sources of slope illusions.

Jeffery et al. discuss the human ability to estimate the slope of surfaces, and leave open the question of whether there is a dissociation between inaccurate conscious judgements of slope and accurate motor actions on the slope. They say that perceptual slope constancy exists - that is, a surface appears to have a relatively constant slope regardless of viewing distance, even though the angle the slope makes with the eye (the optical angle) changes. They also state that observers always overestimate slope, and that this cannot be explained by foreshortening of distance, because that would make downhill slopes appear flatter. Some of these statements can be challenged.

To perceive a geographical scene correctly, the two-dimensional image presented to the eye has to be turned into a threedimensional image, and related to gravity and the angle of regard. Gravity information is obtained through the vestibular system and postural cues, and contributes to knowledge of the angle of regard. Translation to a three-dimensional image depends on distance information, which can be obtained from various binocular and monocular cues at short distances, and at

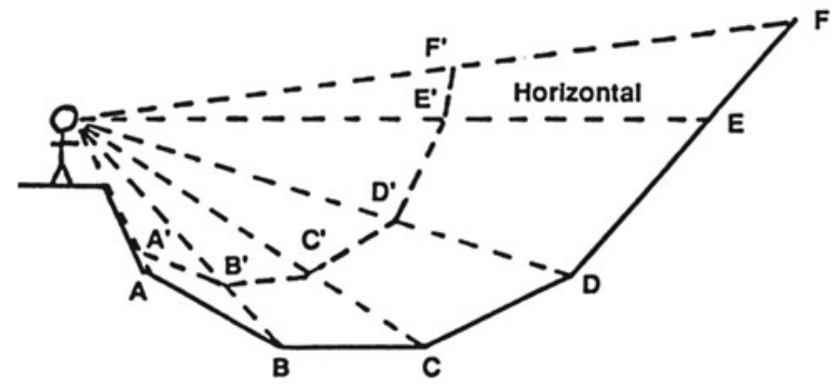

Figure 1 (Ross). Distance foreshortening makes uphill slopes appear too steep (EF to $\mathrm{E}^{\prime} \mathrm{F}^{\prime}$ ) and downhill slopes too shallow (AB to $\mathrm{A}^{\prime} \mathrm{B}^{\prime}$ ). [Reprinted from Ross (1994) with permission]

all distances from the probabilistic relationship between images and their physical sources (Yang \& Purves 2003). Local relationships between different parts of the terrain can produce large slope illusions, or "anti-gravity hills" (Bressan et al. 2003), perhaps by changing the probabilities.

Outdoor observations and experiments using long viewing distances show that apparent slope does vary with the viewing distance and viewing height. Distant uphill slopes appear steeper than near uphill slopes. Distant downhill slopes appear flatter than near downhill slopes, and very distant downhill slopes appear to slope uphill. Skiers at the top of a hill may observe skiers in the valley below apparently skiing uphill. These effects can all be explained by increased foreshortening of distance at further distances (Fig. 1). However, when viewing different parts of the terrain, observers also vary their angle of regard, and the optical angle will change. These effects are particularly marked at short viewing distances and may contribute to different perceptual effects with close viewing.

It is well known that uphill slopes appear steeper from a far than a near distance. Alhazen used foreshortened distance in the 11th century to explain the vertical appearance of distant hills (Sabra 1989). Ross (1974, p. 73) found that skiers gave steeper verbal estimates of an uphill slope from a far viewpoint than when standing on the slope. Creem-Regehr et al. (2004) varied the viewing distance from the base of a hill to 70 meters away, and found an increase in slope estimates from the far distance. Bridgeman and Hoover (2008) had observers stand on a paved hill with an 11 degree slope, and judge the slope when looking up to a cone placed at $1,2,4,8$, and 16 meters uphill from them; their slope estimates increased with the viewing distance. Thus, quite short increases in viewing distance are sufficient to cause an increase in apparent uphill slope, at least when accompanied by a raised angle of regard. In this case the optical angle decreases with the viewing distance, and cannot be used as an explanation of the increase in apparent slope.

The appearance of downhill slopes is more controversial. Unlike uphill slopes, downhill slopes can only be viewed with a lowered angle of regard. For evidence that downhill slopes appear too steep the authors cite Proffitt et al. (1995) and Li and Durgin (2009). However, Li and Durgin conducted a laboratory experiment using very short viewing distances, and found that downhill slopes appeared steeper when viewed further back from the edge of a drop. The increased viewing distance was accompanied by a decreased optical angle and a less depressed angle of regard. For downhill views a smaller optical angle is associated with a steeper slope, and this may explain the effect. The experiment does not compare uphill and downhill views, or deal with large distances. Proffitt et al. used real outdoor hills, viewed from the base or the top. The hills had slopes of from 2 to 34 degrees, but their lengths were not specified. The hills were selected to be near footpaths on a university campus, and to be sufficiently long that the top of the hill was "well above the horizon" when viewed from the base. For an average viewer 
with an eye-height of 1.5 meters, the 2 degree hill would have to be more than 43 meters in length, but the steeper hills could be shorter. The authors also conducted a virtual reality experiment, in which they simulated the measurements for the real 5 degree hill and stated that it was 100 meters in length. The outdoor hill lengths must have varied, but are short in comparison with the mountainous terrain experienced by skiers and hill walkers. The authors found that slopes were overestimated both uphill and downhill, but very slightly more for the steeper downhill views on visual and haptic measures (but not on verbal angular estimates). Similarly, Ross (2006) found no difference between uphill and downhill views on verbal angular estimates, for outdoor slopes between 2 and 23 degrees, with maximum viewing distances of 15 meters for the 23 degrees uphill view, and views of around 50 meters or more for the downhill views. Ross (2010) varied the viewing distance to a bench on a 7 degree downhill slope: She had observers stand on the slope 20 meters above the bench, or view it from a window in a building, giving a viewing distance of 36 meters. The verbal slope estimates were significantly less steep from the higher and further viewpoint. This shows that when viewing distances are well controlled, the flattening effect of a more distant viewpoint can be measured over fairly short distances. Ross (2010) also had observers make angular estimates from the Wallace Monument, 100 meters above a flat plain. The mean estimate for the flat plain was +6.45 degrees, significantly steeper than the estimate of +0.93 degrees given by observers standing on a horizontal footpath, and demonstrating the apparent uphill slope of flat terrain when viewed from a height.

These errors have practical implications for skiers and mountaineers who are trying to decide whether a distant slope is navigable and what effort will be required. Large slope illusions are usually not detected as illusions (Bridgeman \& Hoover 2008) - unless evidence is available, such as skiers gliding uphill or water running uphill. One can learn from experience to guess what is navigable, but experience does not seem to change the false appearance of distant slopes. These observations cannot help the debate on whether there is a dissociation between perception and action, because by the time the traveller reaches the distant slope his perception usually corresponds to reality.

\section{The problem of conflicting reference frames when investigating three-dimensional space in surface-dwelling animals}

doi:10.1017/S0140525X1300054X

\section{Francesco Savelli and James J. Knierim \\ Krieger Mind/Brain Institute, Johns Hopkins University, Baltimore, MD 21218. fsavelli@jhu.edu jknierim@jhu.edu \\ http://krieger.jhu.edu/mbi/knierimlab/}

\begin{abstract}
In a surface-dwelling animal like the rat, experimental strategies for investigating the hippocampal correlates of three-dimensional space appear inevitably complicated by the interplay of global versus local reference frames. We discuss the impact of the resulting confounds on present and future empirical analysis of the "bicoded map" hypothesis by Jeffery and colleagues.
\end{abstract}

At first glance, the question of how the brain represents threedimensional space appears to be a straightforward problem. This review by Jeffery and colleagues highlights instead the thorny nature of the question. The main problem is that most mammals are surface-dwelling animals, and it becomes tricky to devise proper experiments to determine whether these organisms encode space as an isotropic, volumetric representation when they move along surfaces that extend in three dimensions. The "bicoded map" proposal of Jeffery et al. suggests that three- dimensional space is represented not as a true volumetric map, but as a patchwork of diversely oriented, local, quasi-planar maps. Neurophysiological data informing this issue are themselves fragmentary and limited to a few studies that employed complementary strategies (e.g., with respect to the orientation of the animal's body) while addressing different functional correlates of space (e.g., head-direction cells vs. grid cells). Much experimental work is therefore needed to empirically evaluate the debated theoretical scenarios.

The implicit assumption in many experiments is that spatial locations are represented in a global reference frame (e.g., the laboratory room), and that the local apparatus is merely a substrate for the animal to move (actively or passively) in and out of different locations in the global reference frame. However, it is increasingly clear from both behavioral and neurophysiological studies that hippocampal spatial representations are tied more strongly to the local apparatus frame of reference than the global frame of reference when the two frames are translated relative to each other (Knierim \& Hamilton 2011). Hence, all experiments on three-dimensional representations of space that employ surfacedwelling animals must address the problem of interacting reference frames when the 3D volume of the global reference frame is sampled serially via surface-bound navigational epochs. For example, in an experiment not discussed in the target article, place fields were bound to the behavioral track, rather than the $x, y$, or $z$ coordinates of the room, when the track was moved to different locations in both horizontal and vertical axes of the room (Knierim \& Rao 2003). These results suggest that the spatial representation was tied to the local reference frame of the track, rather than to the room coordinates, precluding any strong interpretation regarding whether the cells represented two-dimensional or three-dimensional space in the global reference frame.

One key experiment considered by the authors involved rats running up and down a helical path (Hayman et al. 2011). Firing fields from grid cells and place cells repeated themselves in corresponding positions of all or nearly all laps. Although Jeffery et al. interpret this result as evidence of a diminished representation of space in the $z$-axis, this result could be also interpreted as the laps sharing an identical representation under the control of the frame of reference established by the recurring, stereotypical lap. This interpretation is strongly supported by a study using planar circular and square spiral paths (Nitz 2011): Place fields recurred in corresponding positions of each spiral loop, similar to the repeated firing on the different levels of the helical track. The sizes of the fields grew in lockstep with the concentric loops, except for those fields that recurred at the corners of square spirals. These findings suggest a form of pattern detection operating on the geometrical features of the lap.

The second experiment with neural recordings considered by Jeffery et al. concerned rats climbing on a vertical pegboard (Hayman et al. 2011). Grid cells fired in patterns of vertical stripes, suggesting that the vertical wall was "slicing into" a columnar extension of a classic two-dimensional grid pattern existing on the contiguous floor (which was not sampled in this experiment). This is perhaps the strongest piece of evidence supporting the authors' theoretical proposal, but how would one test this model rigorously? One simple idea is to record the grids on the floor, and then have the rat do the pegboard task as the pegboard is moved to different locations/orientations relative to the floor. As the pegboard slices into different columns, the pattern of stripes should be predictable based on the floor-based grid vertices being intersected by the pegboard. However, once again, the interplay of reference frames may prevent a simple answer. Place cells (Knierim \& Rao 2003; O'Keefe \& Burgess 1996; Siegel et al. 2007) and grid cells (Derdikman et al. 2009; Savelli \& Knierim 2012; Savelli et al. 2008) are strongly influenced by local boundary manipulations, and boundary-responsive neurons are found in multiple hippocampus-related regions (Boccara et al. 2010; Lever et al. 2009; Savelli et al. 2008; Solstad et al. 
2008). If the floor-based grid becomes bound to the pegboard in the first recording, this grid may reset identically at the boundary formed by the base of the pegboard, regardless of where the pegboard is moved. The vertical fields predicted to project over the pegboard from this grid would then remain unchanged, too. This is just one example of a general concern applicable to similar experimental designs in which a surface reference frame is moved relative to another.

The authors make the provocative argument that even animals that truly navigate in three-dimensional volumes - birds, bats, insects, fish - are likely to use a bicoded strategy, rather than a volumetric representation that is isotropic and metric in all three axes. The question can be addressed in these animals without the need of a surface substrate that may be in conflict with a global reference frame. A recent study (Yartsev \& Ulanovsky 2013) did not find elongation along a single common axis in three-dimensional place fields recorded from the hippocampus of freely flying bats, seemingly in contrast with the bicoded map hypothesis.. Future experiments in the same species can test if this result applies to grid cells as well. Regardless of the outcome, it will not be clear whether surface-dwelling creatures will show the same results. Understanding the proper reference frame of the "cognitive map" is one of the crucial problems that must be resolved in order to interpret the data from animals running on surfaces in three-dimensional space, and the reference frames may differ across terrestrial, arboreal, and flying animals.

\section{Just the tip of the iceberg: The bicoded map is but one instantiation of scalable spatial representation structures}

\section{doi:10.1017/S0140525X13000551}

\section{Holger Schultheis and Thomas Barkowsky \\ SFB/TR 8 Spatial Cognition, Universität Bremen, 28359 Bremen, Germany. schulth@sfbtr8.uni-bremen.de barkowsky@sfbtr8.uni-bremen.de http://cosy.informatik.uni-bremen.de/staff/holger-schultheis \\ http://cosy.informatik.uni-bremen.de/staff/thomas-barkowsky}

\begin{abstract}
Although the bicoded map constitutes an interesting candidate representation, proposing it as the predominant representation for threedimensional space is too restrictive. We present and argue for scalable spatial representation structures as a more comprehensive alternative account that includes the bicoded map as a special case.
\end{abstract}

The question of how space, including the third dimension, is represented in the human mind is doubtless of major importance to the behavioral and brain sciences. The bicoded map proposed by Jeffery et al. constitutes an interesting candidate representation. However, it seems unlikely that it captures more than one very specific type of the multitude of spatial representations available to humans. To assume that the bicoded map is more than a special case is to misjudge the nature of spatial representations.

Even in one or two dimensions, mental representations of spatial information are usually neither as metric nor as consistent as assumed in the bicoded map. More often, humans use so-called qualitative representations. Such representations abstract from a continuous property (e.g., distance) by partitioning it and distinguishing only between classes that are defined by these partitions (e.g., near and far in the case of distance; see Forbus 2011). Empirical behavioral (Knauff et al. 1997) and neuroscientific (Sereno et al. 2001) as well as computational (Krumnack et al. 2011) studies have shown that humans are prone to employing qualitative, non-metric spatial representations. Furthermore, human spatial representations suffer from systematic distortions that lead to misrepresentations of, for example, distance (McNamara \& Diwadkar 1997) and orientation (Moar \& Bower 1983). According to these properties, mental spatial representations are often better viewed not as cognitive maps, but as cognitive collages (Tversky 1993): combinations and overlays of qualitative, nonmetric representations of parts and aspects of the overall represented space. In addition, organization of human memory has been found to favor representation structures that are economic in the sense of minimizing storage space and processing requirements for representing a given body of knowledge, both for semantic knowledge generally (Collins \& Quillian 1969) and for spatial knowledge in particular (McNamara 1986; Stevens \& Coupe 1978).

Given these properties of human spatial representations, it seems unlikely that the bicoded map constitutes more than one out of many representations employed by humans. A more comprehensive view on human spatial representations that includes the bicoded map as a special case is provided by our framework of scalable spatial representation structures (Schultheis \& Barkowsky 2011; Schultheis et al. 2007). According to this conception, representations are constructed such that they remain as simple as possible given the current task demands. If the task demand changes, the representations are adapted accordingly and, thus, the representations scale to and with the current task requirements. This on-demand scaling can occur with respect to several aspects of the representations.

First, scaling can occur with respect to the types of spatial knowledge that are represented (e.g., distance, topology, orientation). Evidence from psychological and artificial intelligence research (reviewed in Schultheis et al. 2007) indicates that mental representations of spatial information are best viewed as being composed of several distinct, knowledge-type-specific representation structures. Such knowledge-type-specific representation structures contribute to the scalability of spatial representations. If a certain situation provides or requires only knowledge about orientations between objects, only a representation structure specialized for representing orientation knowledge will be employed. If the consideration of further types of knowledge such as topology becomes necessary, further representation structures that are specialized for representing topological knowledge will be added to the overall spatial representation.

Second, each knowledge-type-specific representation is subject to scaling such that (a) only task-relevant entities are included in the representation and (b) the granularity of the representation, that is, its ability to distinguish between different relations, changes with task demands. Since the granularity of a representation is directly related to how coarsely or finely the represented spatial continuum is partitioned, spatial representations are also scalable in terms of how qualitative or metric they are.

Finally, spatial representations are scalable with respect to the number of dimensions that are encoded. Although a two-dimensional representation may be considered the most common form of spatial representation, one-dimensional representations can be sufficient and even superior to two-dimensional representations in certain contexts (e.g., route representations; MacEachren 1986).

When viewed in the framework of scalable representation structures, the conception of the bicoded map as the predominant mental representation appears too restrictive. It seems implausible to assume that the spatial plane is always represented metrically while the vertical dimension is always exclusively represented qualitatively; which dimensions are represented and how fine-grained the representation is depend on the demands of the current spatial task. For example, when planning a bike trip, the plane may not be represented, but the vertical dimension may be instantiated by a fine-grained (perhaps metric) representation of slope and elevation, because this may constitute the most important information for planning the bike route. On the other hand, if required by the task, humans may construct a full three-dimensional volumetric mental representation. Research on air traffic controllers, for instance, suggests that experienced controllers form a continuous "functional picture of the momentary traffic situation" that allows them "to interpret very 
complex dynamic constellations within a few seconds" (Eyferth et al. 2003, p. 415).

That there is little empirical evidence for metric representations of the vertical dimension or a three-dimensional volumetric representation is, therefore, more indicative of the necessity than of the ability to maintain and employ such representation structures. For many spatial tasks it is sufficient to exclude construction of a representation of the vertical, because the vertical information is (a) irrelevant to the task or (b) partly redundant with horizontal information (e.g., in slanted terrain, moving in certain directions implies certain elevation changes). Due to this lack of a necessity to represent vertical information in much detail (if at all) in many situations, the data reviewed in the target article do not allow unequivocally ruling out alternative representations to the bicoded map. Given the above considerations, the framework of scalable representation structures provides a much more convincing account of human mental spatial representations than does the overly specific bicoded map.

\section{What is optimized in an optimal path?}

\section{doi:10.1017/S0140525X13000563}

\section{Fraser T. Sparks, ${ }^{a}$ Kally C. O’Reilly, ${ }^{b}$ and John L. Kubie ${ }^{a}$ ${ }^{a}$ Department of Cell Biology, The Robert F. Furchgott Center for Neural and Behavioral Science, State University of New York-Downstate Medical Center, Brooklyn, NY 11203; ${ }^{\mathrm{b}}$ Center for Neural Science, New York University, New York, NY 10003. \\ neurosparks@gmail.com kallyco@gmail.com jkubie@downstate.edu www.neuraldynamics.ca http://coronaradiata.net}

Abstract: An animal confronts numerous challenges when constructing an optimal navigational route. Spatial representations used for path optimization are likely constrained by critical environmental factors that dictate which neural systems control navigation. Multiple coding schemes depend upon their ecological relevance for a particular species, particularly when dealing with the third, or vertical, dimension of space.

Modeling the neural systems used for navigating between two locations is ecologically valid when the specific constraints of an environment on a species are accounted for. Navigational routes may be optimized by certain factors in one species, and by different factors in others. Therefore, models of navigational systems within terrestrial, arboreal, aquatic, or aerial species should account for specific factors that dictate route optimization. Jeffery et al. have presented an argument for bicoding, where space in the plane of locomotion is represented independently from that in the orthogonal axis. Although we agree that the evidence presented points toward this coding scheme concept, representation of space may be encompassed by "multi-coding," where multiple systems (not limited to two) compute ecologically valid representations to optimize navigation.

What is the most relevant factor in optimizing a three-dimensional route? Ignoring ecological validity and all factors being equal, the simplest answer is distance. The optimal route is the shortest distance between two points, which is a straight line. In two-dimensional surface navigation, a number of neural computational models propose mechanisms by which the hippocampal formation could compute a straight path (Blum \& Abbott 1996; Burgess \& O’Keefe 1996; Muller \& Stead 1996). Extrapolating from these models to account for the vertical dimension, and maintaining shortest distance as the most optimized factor, calculating the "straight line" is performed by treating the $z$-axis as analogous to the $x$ - and $y$-axes.

Treating the vertical dimension as equivalent to the two horizontal dimensions is not always possible for terrestrial animals when the straight line would require ecologically invalid operations, such as flight or tunneling. To avoid this issue, Jeffery et al. argue that in a three-dimensional environment, the optimal shortest path (straight line) is calculated by using the tangents-to-ground contours. However, other factors that may come into play when optimizing a route should be taken into consideration; for example, metabolic expenditure and journey time.

On flat, unobstructed terrain, the straight-line distance between two locations will yield the path requiring minimal time and energy (metabolic optimization). However, given a terrain with a vertical dimension, the energy expenditure for climbing or descending is complicated and relates to instantaneous slope. Metabolic rate in humans walking on an adjustable incline is a function of speed and slope. For instance, locomotion on a 10 degree slope will approximately double metabolic rate compared with movement at the same speed on a level surface (Silder et al. 2012). Similarly, the metabolic rate of horses on a 10 degree slope increases by approximately 2.5 times (Eaton et al. 1995). Additionally, because climbing is slower than walking on the horizontal, shortest path distance is not equivalent to shortest path time. For species that optimize metabolic cost, models solely optimizing shortest path distance are not ecologically valid when metabolic cost is a primary factor in route navigation. For a terrestrial animal, linear or tangential distance is a poor optimizer for routes in threedimensional space. Though we strongly concur with the overall conclusion of Jeffery et al. our conclusions are based on the inappropriate simplification that the shortest path is the optimal path.

However, the shortest path in three dimensions may be optimized in some species. A number of studies done with spatial representations in the barn owl (Tyto alba) offer insight into how the vertical dimension is processed, and add support to the concept of multi-coding of three-dimensional space (Euston \& Takahashi 2002; Takahashi et al. 2003). Barn owls are known for their ability to hunt in total darkness (Konishi 1973; Payne 1971), and their navigation is guided by a topographical representation of auditory space located in the external nucleus of the inferior colliculus (Knudsen \& Konishi 1978a; 1978b). Neurons within this auditory space map have discrete spatial receptive fields, not unlike place cells in the hippocampus, that result from the computation of inter-aural differences in the level and time-of-arrival of sounds. The most optimal route for the barn owl while hunting is the route that minimizes distance and time to get to the prey.

The auditory spatial map of the barn owl is used as a model of mammalian auditory localization and navigation, and it may be an example of how information about the vertical dimension can be used in conjunction with two-dimensional representations of space. Though terrestrial species may not use auditory information as primary spatial information, arboreal mammals and primate species likely do use this information in forming and utilizing three-dimensional representations.

In conclusion, avoiding a steep direct path by taking a flat, circuitous route is supported by optimizations other than straightline distance. In fact, given the arguments presented here for adding metabolism and time into the optimization of a particular route, we suggest that the shortest path in three-dimensional, as well as two-dimensional, terrains may or may not be a factor used for route optimization. A multi-coding and multi-system scheme for spatial representations in three dimensions is an attractive concept to explain how ecologically relevant factors can contribute to spatial processing.

\section{Grid maps for spaceflight, anyone? They are for free!}

\author{
doi:10.1017/S0140525X13000575
}

\author{
Federico Stella ${ }^{\mathrm{a}}$, Bailu Si, ${ }^{\mathrm{b}}$ Emilio Kropff, ${ }^{\mathrm{c}}$ and Alessandro \\ Treves $^{\mathrm{a}, \mathrm{d}}$
}

${ }^{a}$ Cognitive Neuroscience, SISSA, 34136 Trieste, Italy; ${ }^{b}$ Department of Neurobiology, Weizmann Institute of Science, Rehovot, Israel; ' Laboratory of Neuronal Plasticity, Leloir Institute, CABA, C1405BWE, Buenos Aires, 
Argentina; ${ }^{\mathrm{d} C e n t r e}$ for Neural Computation, Norwegian University of Science and Technology, NO-7491 Trondheim, Norway.

fstella@sissa.it bailu.si@weizmann.ac.il

kropff@gmail.com\#ale@sissa.it

http://people.sissa.it/ ale/limbo.html

Abstract: We show that, given extensive exploration of a threedimensional volume, grid units can form with the approximate periodicity of a face-centered cubic crystal, as the spontaneous product of a self-organizing process at the single unit level, driven solely by firing rate adaptation.

In the target article, Jeffery et al. suggest that a fully three-dimensional representation of space may have not been found in a number of studies, because of the intrinsic computational complexity of $3 \mathrm{D}$ representations, which would make the cost of setting them up too high, and not because of anything to do with the spatial behaviour of the species, such as rats and primates, used in those studies. If so, genuine representations of 3D volumes would be unlikely to be revealed even when moving beyond surface-dwelling species, towards flying or marine animals who appear capable of experiencing 3D space more thoroughly, through three-dimensional navigation.

Clearly, managing directional information in three-dimensional space offers more challenges than on the plane (Finkelstein et al. 2012). Nevertheless, how this may affect the development of a grid representation in three dimensions depends on the mechanisms at work to generate it. If such mechanisms were to rely heavily on head direction inputs, then Jeffery et al. would have a point. If not, there might be space for surprises.

Since 2005, we have been analysing a model for grid cell formation (Kropff \& Treves 2008; Treves et al. 2005) based on a self-organisation process driven solely by firing rate adaptation (head direction information and recurrent connections are needed only to align the grids along common axes; $\mathrm{Si}$ et al. 2012). The model produces grids in two dimensions spontaneously. Individual grid cells average in time the content of spatially modulated inputs, which can be very generic and need not require any specific computation. The emergence of a grid in the firing rate map is induced by the animal's exploration of space, and the final appearance of the grid, its structure, depends on the way the environment is explored ( $\mathrm{Si}$ et al. 2012) and on the topology of the space itself (Stella et al. 2013).

What would this model predict in three dimensions, if the units receive broad spatial inputs modulated also in the third dimension? Individual grids are expressed by the feedforward inputs

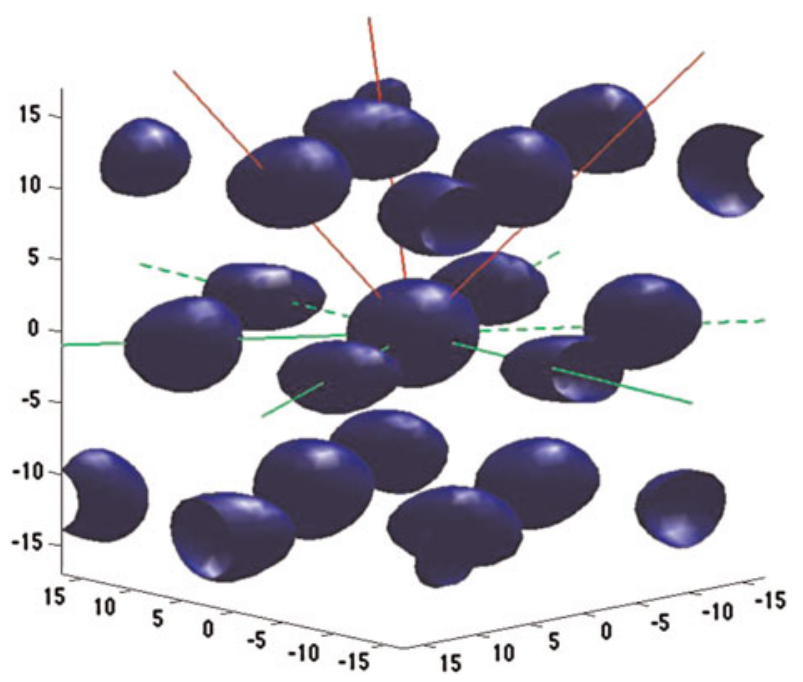

Figure 1 (Stella et al.). The three-dimensional autocorrelogram of a sample unit developing an approximate face-centered cubic (fcc) 3D firing rate map. in the basic model, and the same inputs could "carry" both twodimensional crawling grids and three-dimensional flying grids. We have looked at how the very same model behaves when expanding the original square environment into a cubic one and allowing the simulated animal to fly around for the time usually required for the two-dimensional exploration to generate good grid units.

As in two dimensions, the model produces a regular tiling of space: Its units develop grid fields positioned at the vertices of a lattice uniformly filling the available volume. By computing the 3D autocorrelogram of the spatial activity of these units (Fig. 1), it is apparent that the configuration reached by the fields is the so-called face centered cubic (fcc). In this configuration each field is surrounded by 12 other fields, and all pairs of neighbouring fields have roughly the same distance.

Our model shows how grids in two and three dimensions (or in any number of dimensions) may be produced starting from the very same principles of auto-organization, without increasing costs in higher dimensions. Adaptation provides the means to shape regular forms out of rough and unpolished spatial inputs, and it does so regardless of the topology of the external environment. Without a spatial behaviour engaging the full three-dimensional environment, however, no 3D grid units would appear, as there is no hard-wired or ad hoc designed structure, in our model, to support them.

In the words of Jeffery et al. our model seems to indicate that the absence of three-dimensional grids in rats has an ontogenetic cause (target article, sect 5.2, para. 2): Rats do not possess threedimensional grids because, alas, they have never learned to fly.

\section{What counts as the evidence for three- dimensional and four-dimensional spatial representations?}

\author{
doi:10.1017/S0140525X13000587
}

Ranxiao Frances Wang and Whitney N. Street

Department of Psychology, University of Illinois at Urbana-Champaign, Champaign, IL 61820.

francesw@cyrus.psych.illinois.eduｓtreet1@illinois.edu

http://publish.illinois.edu/franceswang/

Abstract: The dimension of spatial representations can be assessed by above-chance performance in novel shortcut or spatial reasoning tasks independent of accuracy levels, systematic biases, mosaic/segmentation across space, separate coding of individual dimensions, and reference frames. Based on this criterion, humans and some other animals exhibited sufficient evidence for the existence of three-dimensional and/ or four-dimensional spatial representations.

How can one prove that an animal has three-dimensional spatial representations? For navigation in two-dimensional space (e.g., horizontal plane), the classical paradigm is the novel shortcut test: If an animal traverses a route and then returns home by a novel shortcut, this is taken as sufficient (but not necessary) evidence of a 2D spatial representation. A variant of the novel shortcut test is the detour test, where a familiar route is blocked and the animal must navigate around the obstacle to reach the destination. Although novel shortcutting is usually attributed to path integration rather than cognitive mapping, both mechanisms require $2 \mathrm{D}$ spatial representations, and therefore the conclusion regarding dimensionality holds in both cases.

Based on this logic, novel shortcutting in three-dimensional space is sufficient evidence for 3D spatial representations. If an animal traverses a route in three-dimensional space and returns home through a novel shortcut or navigates around an obstacle to reach the goal, then that animal possesses a 3D spatial representation. Among the four candidate models, only the 
volumetric map supports such behavior. Animals using the surface map and the extracted flat map may take shortcuts within the encoded surface, but they cannot return to the appropriate elevation once they are displaced along that dimension. A bicoded map with non-spatial (e.g., contextual) coding of the third dimension allows shortcuts within the surface(s) of the two spatial dimensions, but the animal has to perform a random search along the third dimension until it happens to reach the desired context, because by definition the contextual coding without spatial mapping provides no information about the direction and distance an animal should travel to reach the goal context from a novel context.

The ability to perform novel shortcuts does not necessarily mean high accuracy or efficiency. Performance in navigation tasks varies depending on the species, the perceptual information available, and properties of the trajectory (Loomis et al. 1993; Wang \& Spelke 2003). Differences in accuracy may be due to the quality of the spatial representation and spatial processing. However, lower accuracy cannot be used as evidence against the presence of 3D spatial representations. Instead, the criterion for possessing a 3D representation should be above-chance performance in the third dimension. Similarly, biases occur in spatial judgments of lower dimensions (e.g., Huttenlocher et al. 1991; Sampaio \& Wang 2009) and veridical information is not required for spatial representations. Hence, systematic biases are not evidence against 3D representations.

Moreover, spatial representations do not require integration across dimensions, and none of the four models makes this assumption. Multiple dimensions can be encoded separately, for example, as coordinates for each dimension, as long as the information can be used together for spatial processing. Therefore, separation of one or more dimensions is not evidence against 3D spatial representations. Integration across space is also not required, and fragmentation has been shown in 2D spatial representations (Wang \& Brockmole 2003). All four models are open about whether the entire space is represented as a whole or is divided into pieces/segments, and all can accommodate both mosaic and integrated representations. Thus, segregation of space is not informative on the dimensionality of the spatial representations.

Finally, the dimensionality of spatial representations for navigation needs to be considered in a broader theoretical framework in terms of reference frames and sensory domains. Flexible, efficient navigation does not require an allocentric map. For example, an egocentric updating system can provide the same navigational capabilities as an allocentric cognitive map (Wang 2012; Wang $\&$ Spelke 2000). It is also important to consider visual, proprioceptive, and motor command information for self-motion estimation, which may provide simpler and more efficient computation than the vestibular signals (Lappe et al. 1999; Wang \& Cutting 1999).

As Jeffery et al. discuss in the target article, the dimensionality question has been extended to four dimensions for humans. Although humans probably did not evolve for navigation in fourdimensional space, there are no known inherent constraints in implementing 4D spatial representations with neurons and neural connections, and the existing brain structure may be utilized to accommodate higher-dimensional spatial representations. Therefore, 4D spatial representations cannot be dismissed a priori based on evolution alone and should be treated as an empirical question.

A few studies have examined human 4D spatial representations using variations of the shortcutting task and spatial judgment tasks. Aflalo and Graziano (2008) showed that humans can perform path integration in four-dimensional space with extended training and feedback. Because the movements were orthogonal $\left(90^{\circ}\right.$ rotations $)$ and determined by the observer, updating at each translation and rotation involved only one and two dimensions, respectively, and was relatively easy to compute algebraically within an egocentric reference frame. Nevertheless, representation of a $4 \mathrm{D}$ vector was required, hence the study provided some evidence of 4D representations. Other studies used virtual reality techniques to create visual simulations of $4 \mathrm{D}$ geometric objects (hyper-tetrahedron) and showed that observers could judge the distance between two points and the angle between two lines embedded in the $4 \mathrm{D}$ virtual space (Ambinder et al. 2009). Observers could also estimate novel spatial properties unique to 4D space, such as the size (i.e., hyper-volume) of virtual $4 \mathrm{D}$ objects (Wang, in press), providing further evidence of $4 \mathrm{D}$ spatial representations.

In summary, an animal possesses three-dimensional spatial representations if it can exhibit above-chance performance along the third dimension in a novel shortcut or detour test in 3D space. Based on this criterion, humans (e.g., Wilson et al. 2004), rats (e.g., Jovalekic et al. 2011), and possibly ants (Grah et al. 2007) all encode spatial information about the vertical dimension that is beyond a surface map, extracted flat map, or bicoded map with contextual coding for the third dimension. Thus, these species possess some form of 3D spatial representations for navigation, which may vary in quality and format across species and the individual dimensions may be represented separately and differently. Empirical studies of four-dimensional path integration and spatial judgments suggest that human 4D spatial representations are possible, although the condition and properties of such representations require further investigation.

\section{Are all types of vertical information created equal?}

\author{
doi:10.1017/S0140525X13000599
}

Steven M. Weisberg and Nora S. Newcombe

Department of Psychology, Temple University, Philadelphia, PA 19122.

smweis@temple.edu newcombe@temple.edu

www.spatiallearning.org

Abstract: The vertical component of space occurs in two distinct fashions in natural environments. One kind of verticality is orthogonal-to-horizontal (as in climbing trees, operating in volumetric spaces such as water or air, or taking elevators in multilevel buildings). Another kind of verticality, which might be functionally distinct, comes from navigating on sloped terrain (as in traversing hills or ramps).

Jeffery et al. propose that vertical space is coded using a different set of mechanisms from the head direction, place, and grid cells that code horizontal space. In particular, the authors present evidence that grid cells do not fire periodically as an organism moves along the vertical axis of space, as they do along the horizontal axis, and that the head direction system uses the plane of locomotion as its reference frame. But a challenge for the bicoded model is whether it can account similarly for the different types of information that can be specified in the vertical dimension. We argue that there are two markedly different components of vertical space that provide entirely different spatial information, and therefore are represented differently: orthogonal-to-horizontal (as in multilevel buildings and volumetric space) and sloped terrain.

The vertical dimension, when orthogonal-to-horizontal, can specify contextual information that can be used to reference the appropriate planar map ("what floor am I on"), as well as bodyorientation information ("which way is the axis of gravity"). The former is likely coded categorically in terms of low, high, higher, and so forth, as described by Jeffery et al. in the target article. Theoretically, this information functions no differently from the contextual information provided by rooms with differently colored walls or by the floor numbers outside elevators. The cue (wall color or floor number) specifies the planar cognitive map to which the organism should refer. The vertical dimension can also specify body-orientation information. The organism constantly refers to the plane of its body with respect to 
earth-horizontal as a source of input for the direction of gravity. These cues combine for an organism to determine its elevation with respect to the ground.

In contrast, none of this information is available in sloped environments. Terrain slope can provide a directional cue by specifying compass-like directions (i.e., up $=$ North), which could then orient or augment the planar cognitive map. Note that this processing is distinct from orientation with respect to the direction of gravity, which provides no directional information for the horizontal plane. Recent work conducted in our lab shows that slope is very useful for locating the goal in an otherwise ambiguous environment, and that the directional information it provides is coded with respect to one's own body (Weisberg et al. 2012). Our data suggest that, unlike North, which is an invariant directional cue that does not change based on the direction one faces, terrain slope is coded preferentially as uphill or downhill, depending on one's current facing direction. It is unclear how the vertical dimension with respect to one's own body could provide similar directional information.

Data from our lab also suggest that the way slope information augments the planar map is computationally different from the way the orthogonal-to-horizontal dimension provides context. In a replication of Restat et al. (2004), we found that participants were able to use unidirectional-sloped terrain to make more accurate pointing judgments and sketch maps, compared to the same environment on a flat terrain. However, this was only the case for a simple environment. In a complex environment, only participants with a high self-reported sense of direction used the slope information, while low self-reporters did no better than in the flat condition (Weisberg et al. 2012).

Neither of these experimental effects arises from information provided by the vertical dimension as orthogonal-to-horizontal. That is, the participants in these experiments need not encode their elevation with respect to the ground, but only the direction specified by the gradient of the sloped terrain. The goals of the organism modulate which type of vertical information it encodes. As further evidence of their dissociability, consider how the two possible reference frames might interact (see Fig. 1).

For example, an organism navigating along a sloped terrain could have a categorical representation of the vertical dimension either as it refers to the organism's own body (the gradient at left in the figure) or as it refers to earth-horizontal (the gradient at right). The rat on the left is unconcerned with the increase in elevation, and is similar to the rat on the vertical trunk of the tree in Figure 12 of the target article. The rat on the right, however, is encoding its elevation with respect to some low or high point. Presumably, Jeffery et al. would predict that a rat would encode the vertical dimension with respect to its own body, as in the figure on the left. But in doing so, they may be discounting the information provided by the terrain gradient.

By considering the possible representations of the vertical component of space, one can characterize the qualities of each

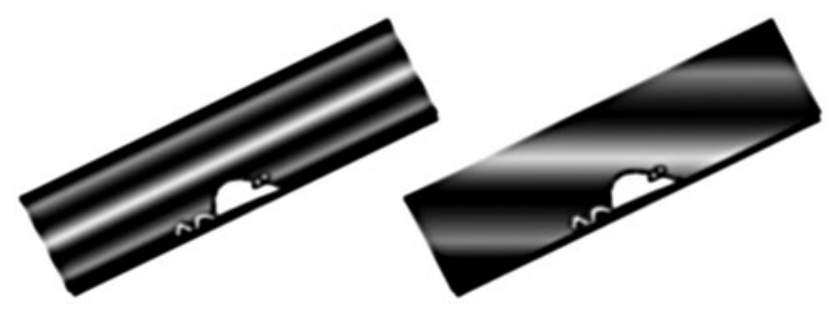

Figure 1 (Weisberg \& Newcombe). Two possible representations of the vertical dimension - one specified with respect to the plane of the organism's body, the other specified by the organism travelling along a sloped surface and therefore changing its elevation. Which of these representations would be predicted by the bicoded model? Are they both categorical? If so, are they functionally similar? (After Jeffery et al.) representation separately. For example, are each of the resultant representations derived from sloped terrain and volumetric space non-metric, or do some of them contain metric properties? If they are non-metric, are they functionally different; susceptible to the same distortions? Whether these representations become integrated and can be used together is an empirical question, but exploring these possibilities creates a richer picture of threedimensional spatial cognition.

\section{ACKNOWLEDGMENTS}

Work on this commentary was supported by grant no. SBE1041707 from the NSF to the Spatial Intelligence and Learning Center.

\section{Map fragmentation in two- and three- dimensional environments}

\section{doi:10.1017/S0140525X13000605}

\section{Homare Yamahachi, May-Britt Moser, and Edvard I. Moser \\ Kavli Institute for Systems Neuroscience, Norwegian University of Science and Technology, NO-7489 Trondheim, Norway. \\ homare.yamahachi@ntnu.no maybm@ntnu.no \\ edvard.moser@ntnu.no}

http://www.ntnu.no/cbm/

Abstract: The suggestion that three-dimensional space is represented by a mosaic of neural map fragments, each covering a small area of space in the plane of locomotion, receives support from studies in complex twodimensional environments. How map fragments are linked, which brain circuits are involved, and whether metric is preserved across fragments are questions that remain to be determined.

Jeffery et al. present an inspiring overview of behavioural and neurobiological studies of neural map formation and navigation in three-dimensional environments. Based on their review of the literature, they suggest that three-dimensional space is "bicoded," meaning that different neural mechanisms are used to map environments in the horizontal and vertical planes. Only the horizontal plane has a metric for distance and direction. The suggested dual coding scheme raises several interesting questions.

The authors propose that three-dimensional space is encoded as a mosaic of contiguous map fragments. These fragments need not be horizontal but can, in principle, have any orientation relative to the Earth's surface. The proposed fragmentation is reminiscent of the decomposition of grid cell maps that takes place in complex environments in two-dimensional space. A few years ago, Dori Derdikman and colleagues measured the effect of environmental compartmentalization on the spatial periodicity in grid cells (Derdikman et al. 2009). Rats were trained to run in a zigzag fashion through 10 consecutive hairpin-shaped flat corridors in a large square enclosure. The compartmentalization of the recording environment disrupted the global grid pattern observed in the same cells in the absence of the corridors. When internal walls were inserted, separate maps were formed for each corridor. Each time the rat turned from one alley to the next, the grid map was reset, and a new sequence of grid fields unfolded. The internal walls broke the grid map into smaller maps, one for each compartment. A similar pattern of sub-maps is visible in entorhinal neurons during navigation in other multi-alley environments (Frank et al. 2000). The sharp transitions at the turning points in these mazes appear to take place simultaneously, in a coherent manner, in grid cells and place cells (Derdikman et al. 2009). Taken together, the studies suggest that complex real-world environments are not represented by a single universal map. Instead, the brain has multiple contiguous grid cell and place cell maps, each covering a small uninterrupted space within the larger environment (Derdikman \& Moser 2010). As argued by Jeffery and colleagues, this 
fragmentation of the cognitive map is probably not limited to the horizontal plane. Fragmentation may be an efficient mechanism for mapping complex environments, both within and across planes.

The fragmentation of the spatial map introduces new issues, however. How are fragments "glued" together at transition points? How does the brain adjust for changes in the slope of the terrain between segments? Is metric information carried over from one fragment to the next? Can animals path-integrate across segments? It is possible, as proposed by the authors, that the integration of the fragments is solved by neural systems and computational algorithms not involving the currently known spatial cell types in the hippocampus or entorhinal cortex. In order to understand how animals navigate in real-world environments, we need to identify the factors that elicit fragmentation and the mechanisms that link one fragment to the next.

We agree that some observations speak in favour of partly specialized mechanisms for encoding space in horizontal and vertical planes, at least in terrestrial animals. This includes the presence of separate cell populations for encoding of pitch and yaw in the lateral mammillary nucleus of the rat (Stackman \& Taube 1998), as well as the finding that head direction cells in rats respond exclusively to variations in the horizontal (yaw) plane, or the plane of locomotion if the surface is tilted (Stackman et al. 2000). The proposed segregation receives further support from a study demonstrating less sensitivity to the vertical dimension in place cells and grid cells in rats (Hayman et al. 2011). As indicated by Jeffery et al., it remains possible that vertical modulation was not expressed in that study because the animals maintained a horizontal orientation during the vertical movement. Moreover, with a predominance of horizontal movements, we cannot exclude that horizontal and vertical dimensions differed with respect to availability of metric cues and that precise fields would emerge also in the vertical direction if sufficient information were available. Jeffery et al. also acknowledge that precise representation of the vertical dimension may require a minimum of prior experience with active movement in threedimensional environments. Most laboratory rats lack such experience. Collectively, the limited existing data raise the possibility that horizontal and vertical dimensions rely on somewhat segregated mechanisms, but the findings do not rule out that experience contributes to such segregation or that the brain, as a whole, has an integrated representation of volumetric space. More experimental work is clearly needed to settle these issues. The target article of Jeffery et al. takes important steps towards identifying critical questions that must be addressed.

\section{Does evidence from ethology support bicoded cognitive maps?}

\section{doi:10.1017/S0140525X13000617}

\section{Shane Zappettini and Colin Allen \\ Program in Cognitive Science and Department of History \& Philosophy of Science, Indiana University, Bloomington, IN 47405. \\ szappett@indiana.edurcolallen@indiana.edu \\ http://mypage.iu.edu/ szappett/ \\ http://mypage.iu.edu/ colallen/}

\begin{abstract}
The presumption that navigation requires a cognitive map leads to its conception as an abstract computational problem. Instead of loading the question in favor of an inquiry into the metric structure and evolutionary origin of cognitive maps, the task should first be to establish that a map-like representation actually is operative in real animals navigating real environments.
\end{abstract}

"The core question for animal navigation," according to Jeffery et al. "concerns what the reference frame might be and how the coordinate system encodes distances and directions within the frame" (sect. 2, para. 2). To answer this question, they hypothesize that a bicoded cognitive map underlies the navigational capacities of animals. Their presumption that navigation requires a cognitive map leads them to conceive of navigation as an abstract computational problem that requires a representational schema powerful enough to encode geometrical features of the familiar environment of the navigating animal. We argue that this presumption leads them to ignore important ethological evidence against cognitive maps.

The work of ethologists is especially relevant to the problem of navigating in a three-dimensional world. Ethologists have produced concrete methods for testing the cognitive map hypothesis during navigational tasks. Despite their passing references to ethology, Jeffery et al. fail to note that most ethologists believe that the experimental evidence runs against the hypothesis that cognitive maps underlie animal navigation (e.g., Bennett 1996; Dyer 1991; Wray et al. 2008; Wystrach et al. 2011). In one such experimental paradigm, the "displacement experiment," an animal is moved prior to navigating to a familiar goal in a familiar range (e.g., see Wehner \& Menzel 1990). If the animal were to take a novel path from the displacement point to the goal, that would count as evidence for map-like navigation. However, typically the animal assumes a heading based on some other kind of information, providing evidence that the animal is using navigational systems (e.g., landmark learning, path integration, systematic search behavior, or a combination thereof) whose representational structure does not preserve the geometrical properties assumed by the cognitive map hypothesis. These studies, unlike those to which Jeffery et al. refer, propose alternate explanations that account for the navigational abilities of animals without presupposing the kinds of complex computation implied by a cognitive map. Simpler navigational strategies (e.g., visual snapshot matching) have been proposed to explain navigation to a goal (Judd \& Collett 1998), with landmarks serving to provide context-dependent procedural information rather than geometric positional information (Wehner et al. 2006). This work shows that an animal may not need to abstract higher-order spatial characteristics of its environment in order to successfully navigate in it. However, without first determining what kind of information an animal is actually using while navigating, it is premature to be investigating the nature of the metric properties of the presumed cognitive map.

We suggest that reconceiving the problem of navigation in a three-dimensional world in ethological terms would shift the focus from describing the computational complexity of the geometric properties of the navigable environment to one wherein the information used by the animal to determine its location relative to some goal is investigated under ecologically valid conditions. By failing to consider the alternative explanations of how animals navigate in real environments, Jeffery et al.'s bicoded map hypothesis puts the hypothetical cart before the evidential horse.

Having ignored evidence against cognitive maps in animals, Jeffery et al. appeal to human behavioral evidence suggesting that the representations utilized in navigation preserve the planar properties of the navigation environment. For example, they summarize several studies indicating that humans are relatively poor at estimating the height of landmarks in a familiar environment (sect. 3.1, para. 7) and other studies suggesting that people overestimate the steepness of hills (sect. 3.2, para. 3). They mention yet another study suggesting that people do poorly when attempting to estimate the direction of objects in a multilayer virtual environment (sect. 3.3, para. 4). What is not clear is how these kinds of judgments are related to the information a navigating animal may use in an online navigational task. Jeffery et al. fail to acknowledge the possibility that these kinds of distance judgments (and their failures) may not be evidence for a bicoded cognitive map precisely because the information contained in such a representation may not be that used 
Response/Jeffery et al.: Navigating in a three-dimensional world

by a navigator in a real navigational task. Differential performance on vertical versus horizontal distance of objects, though perhaps having import for spatial perception in general, may simply be irrelevant to navigation.

We applaud Jeffery et al. for acknowledging that, in general, the problem of navigation is an evolutionary one. However, we find the possibility they endorse to be problematic. They argue that a fully three-dimensional map has never evolved, on the grounds that "[it] is possible that the cost-benefit ratio of the required extra processing power is not sufficiently great" (sect. 5.2 , para. 4), and they suggest that a lower dimensional map-like representation supplemented with unspecified encoding heuristics could achieve successful navigational behavior at lower cost. Parallel reasoning suggests that an even lower dimensional representation (not even a map at all) augmented with appropriate heuristics would have evolved under similar cost constraints. That is to say, given their assumption that evolution works to minimize the cost of a trait (i.e., the cost of a more complex computational system), it becomes plausible that selection would act to produce ever less costly representational systems for navigation, as long as those systems are capable of approximating the same result. This would be consistent with the computationally simpler navigational systems found by ethologists to be sufficient for animals to navigate. In our view, Jeffery et al.'s hypothesis as to the evolutionary origins of the bicoded cognitive map suffers from a deficiency common in attempts to connect the presence of a trait with a story about selection history - that is, that mere consistency between the presence of some trait and some hypothetical selection pressure is the sole measure for plausibility. They have provided a list of how-possibly stories (two of which they reject, one they accept), when what is needed is a how-actually explanation that is properly grounded in the ethological evidence. Finally, we agree that a fully volumetric map may not have evolved, but perhaps because no cognitive map at all is needed for an animal to navigate in a three-dimensional world.

\section{Authors' Response}

\section{A framework for three-dimensional navigation research}

doi:10.1017/S0140525X13001556

\section{Kathryn J. Jeffery, ${ }^{a}$ Aleksandar Jovalekic, ${ }^{b}$ Madeleine Verriotis, ${ }^{\mathrm{c}}$ and Robin Hayman ${ }^{\mathrm{d}}$ \\ ${ }^{a}$ Department of Cognitive, Perceptual and Brain Sciences, Division of Psychology \& Language Sciences, University College London, London WC1H $O A P$, United Kingdom; ${ }^{b}$ Institute of Neuroinformatics, University of Zurich, $\mathrm{CH}$ - 8057 Zurich, Switzerland; ' ${ }^{\circ}$ Department of Neuroscience, Physiology and Pharmacology, University College London, London WC1E 6BT, United Kingdom; 'Institute of Cognitive Neuroscience, Alexandra House, London WC1N $3 A R$, United Kingdom. \\ k.jeffery@ucl.ac.uk ajovalekic@ini.phys.ethz.ch madeleine.verriotis@ucl.ac.uk r.hayman@ucl.ac.uk www.ucl.ac.uk/jefferylab/}

Abstract: We have argued that the neurocognitive representation of large-scale, navigable three-dimensional space is anisotropic, having different properties in vertical versus horizontal dimensions. Three broad categories organize the experimental and theoretical issues raised by the commentators: (1) frames of reference, (2) comparative cognition, and (3) the role of experience. These categories contain the core of a research program to show how three-dimensional space is represented and used by humans and other animals.

\section{R1. Introduction}

Scientists typically approach the problem of how space is represented in the brain using the vehicle of their own preferred model species, whether pigeon, rat, human, or fish. Therefore, it is impressive to see in the commentaries how many issues cut across species, environments, descriptive levels, and methodologies. Several recurring themes have emerged in the commentators' responses to our target article, which we address in turn. These are: (1) Frames of reference and how these may be specified and related, (2) comparative biology and how representations may be constrained by the ecological characteristics and constraints of a given species, and (3) the role of developmental and life experiences in shaping how representations are structured and used. In the following sections we explore each of these themes in light of the commentators' views, and outline research possibilities that speak to each of these.

\section{R2. Frames of reference}

A fundamental issue in spatial representation is the frame of reference, against which positions and movements are compared. The concept of frame is a mathematical construct rather than a real cognitive entity, but it provides a useful way of organizing important concepts in spatial representation. However, the crisp formalism that we have developed in order to treat space mathematically may bear scant resemblance to the brain's actual representation, which has been cobbled together using patchy, multisensory, variable, probabilistic, and distorted information. Nevertheless, it is useful to review the ingredients of a perfect map, before trying to understand how the imperfect and real-world brain may (or may not, as Zappettini \& Allen argue) have approximated this functionality.

Maps can be either metric or non-metric (Fig. R1), the former incorporating distance and direction and the latter (topological maps - see commentary by Peremans \& Vanderelst) using only neighbourhood relationships without reference to precise distances or directions. That said, these parameters are necessarily loosely incorporated into the map, or else the relationship with the real world becomes too disconnected for the map to be useful. We focused on metric maps in our analysis because of the clear evidence of metric processing provided by the recent discovery of grid cells. However, Peremans \& Vanderelst describe how an initially topological map could possibly be slowly metricized through experience.

A metric spatial reference frame, to be useful, needs two features: an origin, or notional fixed point within the frame, from which positions are measured, and some way of specifying directions in each of the cardinal planes. Human spatial maps generally fall into two classes, Cartesian and polar (Fig. R1), which treat these two features slightly differently. A classic Cartesian map has a single plane on which an origin is defined, while directionality is imposed on the plane via the two orthogonal $x$ and $y$ axes, which intersect at this origin. Positions are specified by projecting them onto the axes, yielding two distance parameters per position. Directions are not explicitly represented but can be inferred by reference to the axes. In a polar map, by contrast, direction is explicitly represented. Positions are specified by the distance from the origin and the direction with 
(a) $\mathbf{y}$

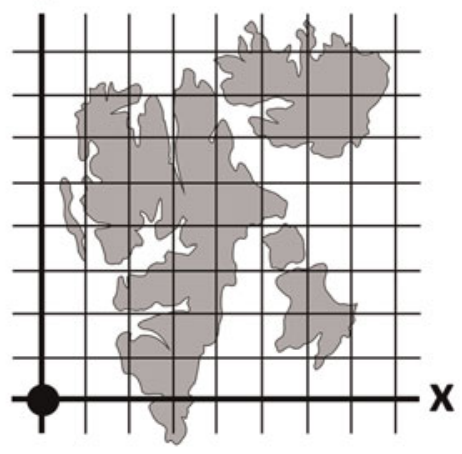

(b)

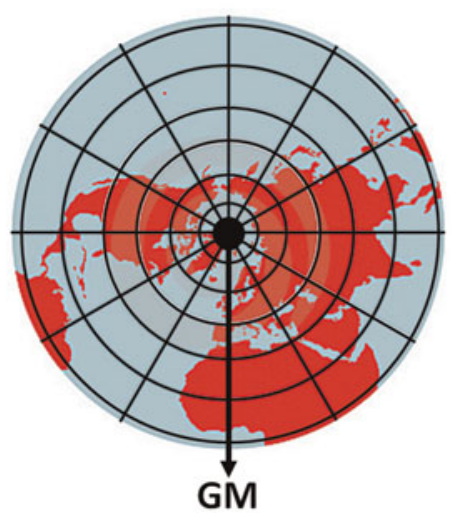

(c)

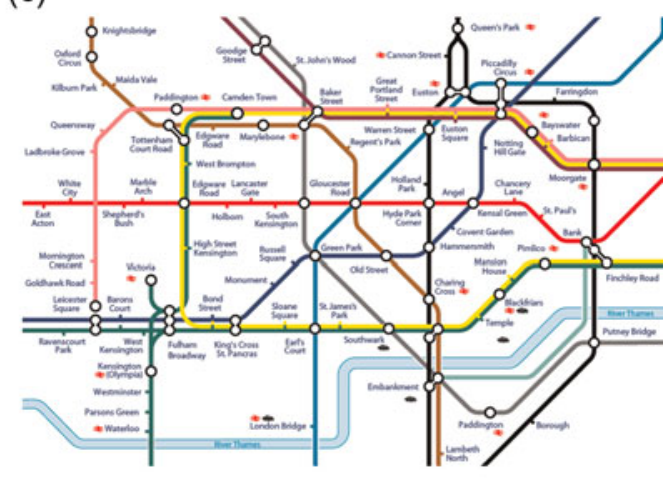

Figure R1. Three types of artificial map representation, two metric and one nonmetric. (a) Cartesian map, showing orthogonal axes $(x$ and $y$ ), an origin (black circle), and a grid with which other places on the map can be metrically specified; (b) polar map, with a specified direction (Greenwich Meridian, GM), an origin (black circle), and a radial grid with which distances in a particular direction relative to the GM can be specified; (c) topological (nonmetric) map - in this case, of the London Underground - in which distances and directions are not explicitly or veridically represented, and in which positions are specified relative to other positions (e.g., "Russell Square is between Holborn and King’s Cross"). Note that the topological map, being nonmetric, does not have an origin or a metric grid.

respect to a reference direction, again yielding two parameters, one specifying distance and one direction.

If a map is a map of the real world, then the frame of reference of the map needs to correspond somehow (be isomorphic) with the world. For example, in a map of the Arctic, the origin on the map corresponds to the North Pole and the reference direction on the map corresponds to the Greenwich Meridian. Spatial inferences made using the map can enable valid conclusions to be drawn about real-world spatial relationships. However, there are many different levels of description of the real world. If a lookout on a ship navigating the polar waters sees an iceberg, then she won't shout "Iceberg, $3 \mathrm{~km}$ South, 20 degrees East!" but rather "Iceberg, $100 \mathrm{~m}$, starboard bow!" - that is, she will reference the object not to the Earth's global reference frame, but rather to the more relevant local reference frame of the ship. Thus, maps can align with the world in many different ways.

In the cognitive science of spatial representation, the global world-referenced frame has come to be called "allocentric," whereas a local self-referenced frame is called "egocentric." As the icebreaker example shows, however, this dichotomy is an oversimplification: If the lookout should then bang her elbow on the gunwale, she will complain not of her starboard elbow but of her right elbow, which of course would become her port elbow if she turned around - "starboard" and "port" are ship-referenced directions and can therefore be egocentric or allocentric depending on the observer's current perception of either being the ship or being on the ship. We will return to the egocentric/allocentric distinction further on.

The final important feature of a reference frame is its dimensionality, which was the primary topic of the target article. Cartesian and polar maps are usually two-dimensional, but can be extended to three dimensions. For both frameworks this requires the addition of another reference axis, passing through the origin and aligned vertically. Now, the position of a point in this space requires three parameters: distance in each of $x, y$, and $z$ for the Cartesian map, and beeline distance plus two directions for the polar map. In the polar map, the first direction is the angle with respect to either/both of the orthogonal directional references in the plane of those axes, and the second direction is the angle with respect to both axes in a plane orthogonal to that.

With these concepts articulated, the question now is how the brain treats the core features of reference frames. We address these in the following order:

1. How are egocentric and allocentric reference frames distinguished?

2. What is the dimensionality of these reference frames?

3. Where is the "origin" in the brain's reference frames?

We turn now to each of these issues as they were dealt with by the commentators, beginning with the egocentric/ allocentric distinction.

\section{R2.1. Egocentric and allocentric reference frames}

When you reach out to pick up a coffee cup, your brain needs to encode the location of the cup relative to your body; the location of the cup relative to the outside world is irrelevant. The frame of reference of the action is therefore centred on your body, and is egocentric. Conversely, if you are on the other side of the room, then planning a path to the cup requires a room-anchored, allocentric reference frame. The fundamental difference between these frames lies in the type of odometry (distance-measuring) required. If the brain's "odometer" is measuring distance between body parts, or movement of one part with respect to another, then the frame is egocentric, and if the measurement is relative to the earth, then it is allocentric. The reason that grid cells have proved so theoretically important is that they perform allocentric odometry-their firing fields are equidistant in earth-centred coordinates - thus proving once and for all that the brain indeed has an allocentric spatial system.

Actions in space typically require more than one reference frame, and these frames therefore need to be related to each other. For example, in reaching for the cup, the movements the hand needs to make are 
constrained by the position of the arm. If reaching for the cup while also walking around the table, then the hand and arm frames need to be updated to account for the relative motion of the cup, and egocentric and allocentric frames must interact. It seems that large parts of the posterior cortex are devoted to these complicated computations, with the parietal cortex being more involved with egocentric space (Andersen \& Buneo 2002) and the hippocampus and its afferent cortices with allocentric space. Where and how these systems interact is not yet known, but as Orban notes, it may be in the parietal cortex.

In our target article we restricted our analysis to allocentric reference frames on the grounds that only allocentric encoding is fully relevant to navigation. The importance to the bicoded model of a possible egocentric/ allocentric unity is that there is better evidence for volumetric spatial encoding in the realm of egocentric action. If egocentric and allocentric space turn out to be encoded using the same representational substrate, the implication is that allocentric space, too, should be volumetric rather than bicoded. Indeed, several commentators question the egocentric/allocentric distinction and contest the exclusion of egocentric spatial processing from a theory of navigation. Orban argues that navigation uses egocentric processes because allocentric and egocentric processing converge on a common neural substrate in the parietal cortex - which is true, but it does not mean they are therefore indistinguishable. Klatzky \& Giudice, referring to studies of humans, ask, "what, then, differentiates the behaviors ostensibly governed by the planar mosaic from human spatially directed actions such as pointing, reaching, over-stepping, and making contact?" - to which we would answer that egocentric actions, such as reaching, do not require allocentrically referenced odometry. They then say, "This exclusion of metrically constrained behaviors from consideration is undermined, however, by the intrinsic ambiguity of frames of reference," by which they presumably mean that humans, at least, can flexibly move between frames of reference. However, movement between reference frames does not imply that the frames are therefore indistinguishable. Klatzky \& Giudice conclude that "lacking reliable behavioral or neural signatures that would allow us to designate actions as egocentric on the basis of their metric demands, it seems inappropriate simply to exclude them from a theory of volumetric spatial representation."

We of course would not want to fully exclude egocentric factors from a complete description of navigation, but it needs to be said that a distinction can be made. As noted earlier, the existence of allocentric odometry, in the form of grid cell activity, shows without a doubt that the brain possesses at least one allocentric spatial system. Given that there are actions that engage grid cells and others that do not, there must be (at least) two forms of spatial representation occurring in the brain: a primarily egocentric one in the parietal cortex that does not require grid cell odometry and a primarily allocentric one in the entorhinal/hippocampal system that does. The question is whether these two systems use the same encoding scheme and/or the same neural substrate. In our view, both are unlikely, although in terms of neural substrate there are clearly close interactions. Therefore, arguing for allocentric isotropy (equivalent encoding in all directions) on the grounds of (possible) egocentric isotropy is unwarranted - they are separate domains.
Of course, there may be more than just these two reference frame systems in the brain - there may even be many. And we accept that the distinction may not be as clear-cut and binary as we made out. Kaplan argues for the possibility of hybrid representations that combine egocentric and allocentric components, and points out that the locomotorplane-referenced form of the bicoded model is in fact such a hybrid. We have some sympathy with this view, as it pertains to the bicoded model, but are not fully persuaded by it. Although it is true that this plane is egocentrically referenced, because it is always under the animal's feet, it is also the case that the locomotor plane is constrained by the environment. So, one could argue that the egocentric frame of reference is forced to be where it is because of allocentric constraints. Thus, the mosaic form of the bicoded model, in which each fragment is oriented according to the local surface, is arguably an allocentrically based, rather than hybrid, model. The distinction may be mostly a semantic one, however.

The egocentric/allocentric distinction can be blurred even in discussions of explicitly allocentric neural representation. In fact, the place cells themselves, long regarded as the prime index of allocentric encoding, are hybrid inasmuch as a place cell fires only when the rat's egocentric reference frame occupies a particular place in the allocentric world. Indeed, it is difficult to think of any example in the existing literature of fully allocentric encoding, in which the position of the organism is truly irrelevant and neurons only encode the relative positions of objects with respect to each other. There may be such neurons, at least in humans, but if they exist, they have not been found yet. Most encoding probably mixes allocentric and egocentric components to a certain degree. Such mixing can cause problems for the spatial machinery. For example, Barnett-Cowan \& Bülthoff speculate that the combination of righting reflexes to maintain an upright head posture during self-motion and object recognition, combined with prior assumptions of the head being upright, may interfere with the brain's ability to represent three-dimensional navigation through volumetric space.

Egocentric and allocentric distinctions aside, within allocentric space one can also distinguish between locally defined and globally defined space, and several commentators have addressed this issue. It is relevant to whether a large-scale representation is a mosaic, a proposal for which Yamahachi, Moser, \& Moser (Yamahachi et al.) advance experimental support, noting that place and grid cell studies show fragmentation of large complex environments based on internal structure (in their case, walls). Howard \& Fragaszy argue that for a sufficiently dense and complex space, such as in a forest, the fragments of the mosaic could in principle become so small and numerous that the map as a whole starts to approximate a fully metric one. We would argue, however, that in order to be fully metric in all dimensions, such a map would need a global 3D directional signal, for which evidence is lacking at present. However, future studies may reveal one, at least in species with the appropriate ecology.

If complex space is represented in a mosaic fashion, then what defines the local reference frames for each fragment? Here it may be that distal and proximal environmental features serve different roles, with distal features serving to orient the larger space and proximal ones perhaps defining local fragments. Savelli \& Knierim observe that local versus global reference frames sometimes conflict. They 
suggest that apparent impoverishment of three-dimensional encoding may result from capture of the activity by the local cues - the vertical odometer is, in essence, overridden by the more salient surfaces of the local environment, which continually act to reset it. For example, consider a helical maze in which an animal circles around on a slowly ascending track, such as the one in the Hayman et al. (2011) study. It may be that the surface - the track - is highly salient, whereas the height cues are much less so, leading to under-representation of the ascending component of the animal's journey. Studies in animals that are not constrained to moving on a substrate, such as those that fly, will be needed to answer the question more generally. The theme of capture by local cues is also taken up by Dudchenko, Wood, \& Grieves (Dudchenko et al.), who note that grid and place cell representations seem to be local, while head direction cells frequently maintain consistency across connected spaces. The implication is that in a truly volumetric, open space, odometry has the capacity to operate in all three dimensions, leading to the creation of an isotropic, volumetric map. Clearly, experiments with other species with other modes of travel and in other environments will be needed to answer the question of whether three-dimensional maps can be isotropic.

\section{R2.2. Dimensionality of reference frames}

Having distinguished between egocentric and allocentric reference frames, we turn now to the issue of dimensionality of these frames, which was the theme of our target article. Although the earlier analysis of reference frames drew a clear distinction between dimensions, the brain does not necessarily respect such distinctions, as pointed out by some of the commentators. Part of the reason for this blurring of the boundaries is that the brain works with information provided by the body's limited sense organs. Thus, it has to cope with the dimension reduction that occurs in the transformation among the real threedimensional world, the two-dimensional sensory surfaces (such as the retina) that collect this information, and the fully elaborated multidimensional cognitive representation that the brain constructs.

Since information is lost at the point of reduction to two dimensions, it needs to be reconstructed again. An example of such reconstruction is the detection of slope using vision alone, in which slope is inferred, by the brain, from visual cues such as depth. Orban explores how the primate visual system constructs a three-dimensional representation of objects in space, and of slope based on depth cues, in the parietal cortex. However, the reconstruction process introduces distortions such as slope overestimation. Orban and Ross both observe that slope illusions vary depending on viewing distance, meaning that the brain has a complicated problem to solve when trying to tailor actions related to the slope. Durgin \& $\mathbf{L i}$ suggest that the action system compensates for such perceptual distortion by constructing actions within the same reference frame, such that the distortions cancel.

Perception of three-dimensional spaces from a fixed viewpoint is one problem, but another quite different one concerns how to orchestrate actions within 3D space. For this, it is necessary to be able to represent the space and one's position within it-problems that are different for egocentric versus allocentric space.
Taking egocentric space first: How completely threedimensional is the $3 \mathrm{D}$ reconstruction that the brain computes for near space (i.e., space within immediate reach)? Several commentators argue in favour of a representation having full three-dimensionality. Orban outlines how the parietal cortex, generally believed to be the site of egocentric spatial encoding (Galati et al. 2010), is well specialized for representing space in all three dimensions, while Badets suggests that the spatial dimensions may be mapped to a common area in the parietal cortex that integrates according to a magnitude-based coding scheme (along with other magnitudes such as number). Lehky, Sereno, \& Sereno (Lehky et al.) agree that primate studies of the posterior cortex in egocentric spatial tasks show clear evidence of three-dimensional encoding. They say, "While the dimensionality of space representation for navigation [our emphasis] in primates is an important topic that has not been well studied, there are physiological reasons to believe that it may be three-dimensional," although they do not outline what those reasons are. By contrast, Phillips \& Ogeil argue that even egocentric space is bicoded. First they appeal to evidence from perceptual illusions and neglect syndromes to show that vertical and horizontal spaces are affected differently. Then they turn to a theoretical analysis of the constraints on integration of vertical and horizontal space, and problems such as chaotic dynamics that can result from attempts at such integration. It therefore seems that more research is needed to understand the representation of near space, and whether or not it is different in different dimensions (anisotropic).

Turning to allocentric space, the issue of the dimensionality was explored extensively in the target article and again in the commentaries. The core questions concerning encoding of the third allocentric dimension have to do with whether it is encoded, and if so, how, and how it might (or might not) be integrated with the other two.

The question of whether it is encoded is addressed by several commentators. Weisberg \& Newcombe make the important point that "vertical" comes in (at least) two forms, orthogonal to horizontal or orthogonal to the animal. They suggest that the different types of vertical may have different contributions to make to the overall encoding of the situation. For example, terrain slope may be useful in, for example, helping orient the local environment, but this information would be lost if vertical encoding were earth-horizontal-related. They suggest that both types of vertical may be encoded, and may or may not be integrated.

Other commentators focus on the encoding of volumetric (rather than slanting planar) three-dimensional space, and speculate about how such encoding may be achieved. Powers argues, on the basis of studies in robotics and engineering, for dimension reduction as a means of achieving coding efficiency by reducing redundancy (i.e., what Carbon \& Hesslinger call "empty cells" that contain no information but take up representational space). In this light, the bicoded model would seem to offer such efficiency. Carbon \& Hesslinger agree, arguing that a two-dimensional map with alternative information for the vertical dimension should suffice for most things, and they draw on comparative studies to support this. This seems a very reasonable proposition to us. For a surface-dwelling animal traversing, say, hilly terrain, there is only one $z$-location for each $x-y$ position, and so the 
animal could in principle navigate perfectly well by computing only the $x-y$ components of its journey. The only drawback would be in, say, comparing alternative routes that differed in how much ascent and descent they required. However, this could be encoded using some other metric than distance-for example, effort-much like encoding that one route goes through a boggy swamp and the other over smooth grassland. The navigation system does not necessarily have to perform trigonometry in all three dimensions to compute an efficient route across undulating terrain. For volumetric space, the problem becomes slightly less constrained because there are unlimited $z$-locations for every $x-y$ point. However, again, it might be possible to navigate reasonably effectively by simply computing the trigonometric parameters in the $x-y$ (horizontal) plane and then factoring in an approximate amount of ascent or descent.

An interesting related proposal is put forward by Schultheis \& Barkowsky, that representational complexity could be expanded or contracted according to current need. According to their concept of scalable spatial representations, the detail and complexity of the activated representation varies with task demands. They suggest that the vertical dimension might be metrically encoded in situations that demand it (e.g., air traffic control) but not in situations that do not (e.g., taxi driving). Dimensionality of encoding might also be affected not just by task demands but by cognitive load. By analogy with the effects of load on attentional processing (Lavie 2005), one might suppose that in conditions of high cognitive load (such as a pilot flying on instruments), the degree to which all dimensions are processed might be restricted. Such processing restrictions could have important implications for training and instrument design for air- and spacecraft.

Wang \& Street move in the opposite direction from dimension reduction. They argue not only that the cognitive representation of allocentric space is a fully fledged three-dimensional one, but also that with the same neural machinery it is possible to encode four spatial dimensions. They support their assertion with data showing that subjects can perform at above-chance levels on path-completion tasks that cross three or four dimensions, something they say should be possible only with a fully integrated volumetric (or perhaps "hypervolumetric") map. Our view on this suggestion is that it is a priori unlikely that an integrated 4D map could be implemented by the brain because this would require a $4 \mathrm{D}$ compass, the selection pressure for which does not exist in a 3D world. Our interpretation of the 4D experiment is that if subjects could perform at above-chance levels, then they would do so using heuristics rather than full volumetric processing, and by extension the above-chance performance on 3D tasks may not require an integrated 3D map either. The experimental solution to this issue would be to identify neural activity that would correspond to a $4 \mathrm{D}$ compass, but computational modelling will be needed to make predictions about what such encoding would look like.

Given general agreement that there is some vertical information contained in the cognitive map (i.e., the map has at least 2.5 dimensions), the next question concerns the nature of this information - is it metric, and if not, then what is it? A fully volumetric map would have, of course, complete metric encoding of this dimension as well as of the other two, and Wang \& Street argue for this. Others such as
Savelli \& Knierim and Yamahachi et al. argue that we will not know for sure until the relevant experiments are done with a variety of species and in a variety of environmental conditions. A number of commentators agree with our suggestion, however, that the vertical dimension (or, in the mosaic version of the bicoded model, the dimension orthogonal to the locomotor plane) is probably not encoded metrically. There are a variety of opinions as to what the encoded information is however. Phillips \& Ogeil suggest that the other dimension is "time" rather than altitude, although it is not clear to us exactly how this would work. Nardi \& Bingman support the proposal that "effort" could be a height cue. Sparks, O'Reilly, \& Kubie (Sparks et al.) develop the notion of alternative factors furtherthey discuss the issue of optimization during navigation, pointing out that the shortest path is not necessarily optimal and that factors such as energy expenditure are also important. They suggest a "multi-coding" (as opposed to merely a "bicoding") scheme to take into account these other factors. And as mentioned earlier, Weisberg \& Newcombe suggest that there are two types of vertical information, a true alignedwith-gravity vertical and another that is related to terrain slope, raising the issue of how these two forms may be related, if at all. Thus, it appears that a variety of stimulus types might serve to input into the encoding of vertical space.

The final question regarding the vertical dimension is whether-assuming it is represented at all - it is combined with the horizontal ones to make a fully integrated, volumetric map. Burt de Perera, Holbrook, Davis, Kacelnik, \& Guilford (Burt de Perera et al.) refer to studies of fish to argue that vertical space, although in their view coded metrically, is processed separately from horizontal space. They suggest some experiments to explore whether this is the case, by manipulating animals' responses in the vertical dimension and showing that these show evidence of quantitative encoding (e.g., by obeying Weber's law). However, similar results could also be found if the animal were using some kind of loose approximation to a metric computation (e.g., climbing to a certain level of exhaustion), and so the experiments would need to be carefully designed so as to show operation of true odometry.

Before leaving the issue of reference frames, it is worth briefly examining the little-discussed issue of how the brain may define an "origin" within these frames.

\section{R2.3. The origin of reference frames}

As noted in the earlier analysis of reference frames, a metric map needs an origin, or fixed point, against which the parameters of the space (distance and direction) can be measured. The question of whether the brain explicitly represents origins in any of its reference frames is completely unanswered at present, although a notional origin can sometimes be inferred. To take a couple of examples among many, neurons in the visual cortex that respond proportionally to distance from the eye (Pigarev \& Levichkina 2011) could be said to be using the retina as the "origin" in the antero-posterior dimension, while neurons that are gain modulated by head angle (Snyder et al. 1998) are using the neck as the "origin" (and straight ahead as the "reference direction"). Whether there exist origins for the other egocentric reference frame, however, is less clear.

For allocentric space, the origin becomes a more nebulous concept, although in central place forager species, 
such as rats, the central place (the nest) may constitute an "origin" of sorts. However, it may be that the brain uses not a fixed point as its reference so much as a fixed boundary. Place and grid cells, for example, anchor their firing to the boundaries of the local environment, moving their firing fields accordingly when the boundaries are moved en bloc. Furthermore, at least for grid cells, deformation (as opposed to rigid translation) of these boundaries in some cases leads to a uniform deformation of the whole grid with no apparent focus (Barry et al. 2007), suggesting that the whole boundary contributes to anchoring the grid. Hence, it is not obvious that there is a single pointlike origin in the entorhinal/hippocampal representation of allocentric space. However, it could be that the place cells themselves in essence provide multiple origins their function may be to anchor spatially localized objects and events to the reference frame defined by the boundaries and grids. It may be that the simple, singular notion of origin that has been so useful in mathematics will need to be adapted to accommodate the brain's far more nebulous and multifarious ways of working.

\section{R3. Comparative studies}

We turn now to the ways in which studies from different species, with different evolutionary histories and ecological constraints, have contributed to our understanding of spatial encoding. Principles derived for one species may not apply to a different species in a different environment using a different form of locomotion. Orban points out, for example, that rodent and primate brains are very different, and that there are cortical regions in primates that do not even have homologues in rodents. Hence, extrapolation from rodents to primates must be done with care, a point with which we agree.

The importance of comparative studies in cognitive science lies in understanding how variations in body type, ecological niche, and life experience correlate with variations in representational capacity. This information tells us something about how these representations are formed-what aspects of them are critical and what aspects are just "add-ons" that evolved to support a particular species in a particular ecological niche. While comparative studies of three-dimensional spatial cognition are rare, they promise to be particularly revealing in this regard.

\section{R3.1. Studies of humans}

Humans are, naturally, the species that interests us the most, but there are important constraints on how much we can discover about the fine-grained architecture of our spatial representation, until non-invasive single neuron recording becomes possible. There are, however, other means of probing encoding schemes, such as behavioural studies. Longstaffe, Hood, \& Gilchrist (Longstaffe et al.) and Berthoz \& Thibault describe the methods that they use to understand spatial encoding in humans over larger (non-reachable) spaces. However, Carbon \& Hesslinger point out that experimental studies need to take into account the ecological validity of the experimental situation, something which is sometimes difficult to achieve in laboratory studies in which natural large-scale threedimensional spaces are rare. Virtual reality (VR) will likely help here, and future studies will be able to expand on previous research in two-dimensional spaces to exploit the capacity of VR subjects to "float" or "fly" through 3D space. This could be used not just during behavioural studies but also in neuroimaging tasks, in which the participating brain structures can be identified.

Some commentators describe the human studies in real (non-virtual) 3D spaces that have recently begun. Hölscher, Büchner, \& Strube (Hölscher et al.) extend the anistropy analysis of our original article to architecture and environmental psychology, and note that there are individual differences in the propensity to form map-based versus route-based representations. Pasqualotto \& Proulx describe prospects for the study of blind people, whose condition comprises a natural experiment that has the potential to help in understanding the role of visual experience in shaping spatial representation in the human brain. Klatzky \& Giudice argue that humans are ecologically three-dimensional inasmuch as their eyes are raised above the ground and their long arms are able to reach into $3 \mathrm{D}$ space, meaning that it is adaptive to represent local space in a fully three-dimensional manner. Indeed, evidence supports that local (egocentric) space is represented isotropically in primates, a point made also by Orban, though contested by Phillips \& Ogeil.

A final important facet of human study is that humans have language, which provides insights unavailable from animal models. In this vein, Holmes \& Wolff note that spatial language differentiates horizontal axes from vertical axes more than it differentiates the horizontal ones from each other, thereby supporting the notion of anisotropy in how space is encoded. However, as discussed in the next section, the degree to which such anisotropy could be due to experience rather than innate cognitive architecture is still open for debate.

\section{R3.2. Studies of nonhumans}

Moving on to nonhuman animals, Carbon \& Hesslinger review the various ways in which the spatial problems faced by animals of a variety of different ecologies reduce to a common set of mainly surface-referenced problems. However, not all commentators agree that a planar or quasi-planar map is supported by comparative studies. Orban observes that the nonhuman primate brain is much closer to the human than to the rodent brain on which much neurobiological work has been done, and notes that several parietal areas in primates are specialized for spatial representation. Howard \& Fragaszy discuss the issue of nonhuman primates moving in a dense arboreal lattice. They suggest the use of laser scanning technology (LiDAR) to map out the movements of primates to determine how their movements are constrained (or at least informed) by the structure of the complex spaces through which they move.

Nonhuman primates are relatively close to humans in evolutionary terms, and commonalities in spatial behaviour might reflect this. Useful insights can therefore be gleaned by moving phylogenetically further away, to try and discern which features are ancient and foundational, and which are more species-specific. Moss advocates bats for the study of three-dimensional spatial encoding. Not only can bats fly, and thus move through space in an unconstrained way, but they also have a sense - echolocation - unavailable to most mammals. This provides a tool for understanding 
Response/Jeffery et al.: Navigating in a three-dimensional world

how a supramodal cognitive representation can be formed from sensory inputs that show local (species-specific) variation. Electrolocation and magnetic sense are additional sensory systems in other species that may also be informative here.

Moving even further away from humans, non-mammalian vertebrates, such as birds, and invertebrates have been valuable in providing comparative data that reveal both commonalities and differences in how different organisms represent space. Zappettini \& Allen take issue with the notion that there is a map-like representation operating in nonhuman animals, suggesting that this is putting "the hypothetical cart before the evidential horse." The debate about the operation of cognitive maps in animals is a longstanding one that is too complex to fully revisit in this forum. However, we would argue, in defence of our proposition, that the existence of grid cells is evidence of at least some kind of map-like process. That there are neurons in the brain that respond to distances and directions of travel is incontrovertible proof that rodent brains - and therefore likely the brains of other mammals, at leastcompute these parameters. Regardless of whether one considers a representation incorporating distance and direction to necessarily be a map, it is nevertheless valid to ask whether encoding of these parameters extends into all three dimensions. To this extent we hope that common ground can be found with the map-skeptics in devising a program of study to explore real-world spatial processing. Zappettini \& Allen also object to our use of evolutionary selection arguments to defend the bicoded hypothesis over the volumetric one. We agree that such arguments are weak and serve only to suggest hypotheses, and not to test them. However, suggesting hypotheses was the main aim of our target article, given the paucity of hard data available at present.

Other comparative biologists have been more relaxed about the notion of a map-like representation underlying navigational capabilities in nonhumans. Citing studies of avian spatial encoding, Nardi \& Bingman suggest that taxonomically close but ecologically different species would be useful comparators - for example, chickadees (inhabiting an arboreal volumetric space) versus nutcrackers (storing food on the ground). Burt de Perera et al. use studies of fish to argue that vertical space, although coded separately from horizontal space, is nevertheless encoded metrically. This finding is interesting because fish can-unlike humans-move in an unconstrained way through 3D space, and would in theory benefit from an integrated 3D map if such had evolved. That the fish map is evidently not integrated in this way suggests that maybe such integrated maps never did evolve. Dyer \& Rosa suggest that study of simple organisms such as bees can help reveal the ways in which complex behaviours can arise from simple building blocks, particularly when plasticity is added to the mix. They agree, however, that evidence suggests that in bees, too, space is represented in a bicoded fashion.

In summary, then, studies in comparative cognition may be tremendously informative in the unravelling of the neurocognitive representation of three-dimensional space, both by revealing which features are central and which are peripheral "add-ons," and by showing how different ecological constraints and life experiences contribute to shaping the adult form of the representation.

\section{R4. Experience}

The final major theme in the commentaries concerned the possible role of experience in shaping spatial representations. Note that just because experience correlates with encoding format (bicoded, volumetric, etc.), it does not necessarily follow that it caused that format. For example, an animal that can fly will have (perhaps) an ability to encode 3D space, and also a lifetime's experience of moving through 3D space, but the encoding may have been hard-wired by evolution rather than arising from the individual's own life experience. Yamahachi et al. stress that more research is needed to determine the role of experience in shaping the structure of the map. This could be done by, for example, raising normally 3D-exploring animals in a restricted, 2D space to see whether the encoding type changes.

Experience, if it affects encoding at all, can act in two broad ways: It can operate during development to organize the wiring of the underlying neural circuits, or it can operate on the fully developed adult brain, via learning, to enable the subject to acquire and store new information. These two areas will be examined in turn.

\section{R4.1. Developmental experience}

It is now clear that infants are not blank slates on which all their adult capacities will be written by experience, but are born with many of their adult capacities having already been hard-wired, under genetic control. That said, it is also clear that developmental experience can shape the adult brain. How experience and hard-wiring interact is a matter of considerable interest.

Experience during ontogeny can shape development of a neural structure by affecting neural migration and axonal projections, or else, subsequently, by affecting dendritic connections and synapse formation. Migration and axon development are generally complete by the time a developing animal has the ability to experience three-dimensional space and so are unlikely to affect the final structure of the spatial representation. On the other hand, dendritic and synaptic proliferation and pruning processes are plentiful during infant development-however, they also take place to an extent during adulthood, when they come under the rubric of "learning." It is evident, therefore, that development and learning show a considerable overlap.

Some sensory systems show critical periods in infancy during which experience is necessary for normal brain development, and interruption of which can cause lifelong impairment. Vision in mammals is a prime example of such experience-dependent plasticity: Monocular deprivation or strabismus (squint) occurring during the critical period, but not during adulthood, can permanently affect connection formation in the thalamus and visual cortex, resulting in adult visual impairment (amblyopia; Morishita \& Hensch 2008). A natural question arising from studies of threedimensional encoding in animals, then, is whether infant experience can affect the formation of the adult cognitive representation.

Recent studies have suggested that head direction cells, place cells, and grid cells start to operate in adult-looking ways very early in infancy in rat pups. In two studies published together in a 2010 issue of Science (Langston et al. 
2010; Wills et al. 2010), cells were recorded from the first day the pups started exploring, 16 days after birth. Head direction cells already showed adult-like firing and stability even though the pups had had very little experience of movement and only a few days of visual experience. Place cells were also apparent from the earliest days of exploration, although their location-specific firing improved in coherence and stability with age. Grid cells were the last cell type to mature, appearing at about 3-4 weeks of age, but when they did appear they showed adult-like patterns of firing with no apparent requirement for experiencedependent tuning. Thus, the spatial system of the rat seems to come on-stream with most of its adult capabilities already present, suggesting a considerable degree of hardwiring of the spatial representation.

Is this true for vertical space as well? The experiment of Hayman et al. (2011) showed a defect in vertical grid cell odometry, and perhaps this defect is due to the limited $3 \mathrm{D}$ experience that rats in standard laboratory cages have had. However, if extensive experience of $2 \mathrm{D}$ environments is not needed for the spatial cells to operate in the way that they do, as is manifestly the case, then experience of $3 \mathrm{D}$ environments may not be needed either. Thus, the question of whether infant experience is necessary for adult cognitive function in the spatial domain is one that awaits further data for its resolution.

Although the experiments have yet to be done, some theoretical analysis of this issue is beginning. Stella, Si, Kropff, \& Treves (Stella et al.) describe a model of grid formation in two dimensions that arises from exploration combined with simple learning rules, and suggest that this would extend to three dimensions, producing a facecentered cubic array of grid fields. We would note here that such a structure would nevertheless be anisotropic, because the fields do not form the same pattern when transected in the horizontal plane as in the vertical. While such a grid map would be metric, Peremans \& Vanderelst discuss a theoretical proposal whereby a non-metric, topological map could be built up from experience, by recording approximate distances travelled between nodes and using these to build a net that approximates (topologically) the real space. They note that this would look anisotropic because of differential experience of travel in the different dimensions, even though the same rules operate in all three dimensions. This is an interesting proposal that predicts that animals with equal experience in all three dimensions should produce isotropic spatial representations, something that may be tested during recordings on flying animals such as bats.

\section{R4.2. Adult experience}

As noted above, there are considerable overlaps in the biological processes supporting infant development and adult learning, because the processes of dendritic arborization and synaptic pruning that support experience-dependent plasticity operate in both domains (Tavosanis 2012). The question arises, then, as to whether adult experience can shape the structure of a complex cognitive representation such as that of space.

Dyer \& Rosa suggest that in bees, although movement in the vertical dimension (determined using optic flow) is usually represented with a lower degree of precision, brain plasticity could allow the experience-dependent tuning of responses such that precision in this dimension could be increased. In this way, the organisms can learn to use navigationally relevant information particular to a given environment. This does not mean, however, that they learn a different mechanism for combining information. Moreover, Dyer \& Rosa agree that the evidence supports the notion of a bicoded structure to spatial processing in bees, even with experience.

Experiments involving human subjects have provided insights into how experience might constrain spatial representation. Berthoz \& Thibault discuss how the learning experience during exploration of a multilayer environment can shape the way in which subjects subsequently tackle navigation problems in that environment (Thibault et al. 2013). Pasqualotto \& Proulx explore the effects that visual deprivation (through blindness) can have on the adult spatial representation that forms. They suggest that congenitally blind individuals may not exhibit quasi-planar spatial representation of three-dimensional environments, positing that the absence of visual experience of the 3D world may shape how spatial encoding occurs. This is a matter that awaits further study, as 3D spatial encoding has not yet been explored in subjects who are blind. These authors suggest several ways in which such studies might be conducted, using sensory substitution devices (which convert visual information into tactile or auditory) in order to create $3 \mathrm{D}$ virtual reality environments. The difference between subjects who are congenitally blind and those who had had visual experience in early life will be particularly interesting here.

Experience may play a role in how normal-sighted subjects perceive and interpret the spatial world, too. Bianchi \& Bertamini discuss the interesting errors that human subjects make when predicting what will be visible in a mirror as they approach it, and show that the errors are different for horizontal versus vertical relationships. Tellingly, adults make more errors than children. These findings suggest that experience may play a part in generation of errors adults have more experience of crossing mirrors from one side to the other than from top to bottom, and more experience of passing in front of mirrors generally than children do. This may perhaps be a case where experience diminishes the capacity to accurately represent a space, because adults have enhanced ability to make inferences, which they do (in this case) erroneously.

While it is certainly the case that experience is likely to inform the adult cognitive representation of space, our hypothesis is that experience is not necessary to construct it. This is because the rodent studies described earlier suggest a high degree of representational complexity even in animals that have had impoverished spatial life experience. Future research in animals reared in complex 3D environments will be needed to determine the extent to which experience is needed for formation and refinement of the brain's map of 3D space.

\section{R5. Conclusion}

It is apparent from the commentaries on our target article that there are many questions to be answered concerning the relatively nascent field of three-dimensional spatial encoding. The most salient questions fall into the categories outlined in this response, but no doubt more will emerge as 
References/Jeffery et al.: Navigating in a three-dimensional world

spatial cognition researchers start to tackle the problem of representing large-scale, complex spaces. The work will require an interplay between behavioural and neurobiological studies in animals and humans, and the efforts of computational cognitive scientists will be needed to place the results into a theoretical framework. The end result will, we hope, be a body of work that is informative to many disciplines involved both in understanding natural cognitive systems and also in building artificial spaces and artificial devices.

\section{ACKNOWLEDGMENTS}

The work was supported by grants to Kate Jeffery from the Wellcome Trust (GR083540AIA), the European Commission's Seventh Framework Programme (SPACEBRAIN), the Medical Research Council (G1100669), and the Biotechnology and Biological Sciences Research Council (BB/J009792/1), and by a grant to Axona Ltd from the European Commission's Seventh Framework Programme (SPACEBRAIN).

\section{References}

[The letters " $a$ " and " $r$ " before author's initials stand for target article and response references, respectively]

Abdollahi, R. O., Jastorff, J. \& Orban, G. A. (2012) Common and segregated processing of observed actions in human SPL. Cerebral Cortex. E-pub ahead of print, August 23, 2012. [GAO]

Aflalo, T. N. \& Graziano, M. S. (2008) Four-dimensional spatial reasoning in humans. Journal of Experimental Psychology: Human Perception and Performance 34(5):1066-77. [aKJJ, RFW]

Allen, G. L. (1999) Spatial abilities, cognitive maps, and wayfinding: Bases for individual differences in spatial cognition and behavior. In: Wayfinding behavior: Cognitive mapping and other spatial processes, ed. R. G. Golledge, pp. 46-80. Johns Hopkins Press. [CCC]

Ambinder, M. S., Wang, R. F., Crowell, J. A., Francis, G. K. \& Brinkmann, P. (2009) Human four-dimensional spatial intuition in virtual reality. Psychonomic Bulletin and Review 16(5):818-23. [RFW]

Andersen, R. A. \& Buneo, C. A. (2002) Intentional maps in posterior parietal cortex. Annual Review of Neuroscience 25:189-220. [GAO, rKJJ]

Angelaki, D. E. \& Cullen, K. E. (2008) Vestibular system: The many facets of a multimodal sense. Annual Review of Neuroscience 31:125-50. [aKJJ]

Angelaki, D. E., McHenry, M. Q., Dickman, J. D., Newlands, S. D. \& Hess, B. J. (1999) Computation of inertial motion: Neural strategies to resolve ambiguous otolith information. Journal of Neuroscience 19(1):316-27. [aKJJ]

Aoki, H., Ohno, R. \& Yamaguchi, T. (2005) The effect of the configuration and the interior design of a virtual weightless space station on human spatial orientation. Acta Astronautica 56:1005-16. [aKJJ]

Avarguès-Weber, A., Dyer, A. G., Combe, M. \& Giurfa, M. (2012) Simultaneous mastering of two abstract concepts by the miniature brain of bees. Proceedings of the National Academy of Sciences USA 109:7481-86. [AGD]

Avery, G. C. \& Day, R. H. (1969) Basis of the horizontal-vertical illusion. Journal of Experimental Psychology 81:376-80. [JGP]

Avraamides, M., Klatzky, R. L., Loomis, J. M. \& Golledge, R. G. (2004) Use of cognitive vs. perceptual heading during imagined locomotion depends on the response mode. Psychological Science 15:403-408. [RLK]

Badets, A., Koch, I. \& Toussaint, L. (2013) Role of an ideomotor mechanism in number processing. Experimental Psychology 60:34-43. [ABa]

Badets, A. \& Pesenti, M. (2011) Finger-number interaction: An ideomotor account. Experimental Psychology 58:287-92. [ABa]

Baker, G. C. \& Gollub, J. G. (1996) Chaotic dynamics: An introduction. Cambridge University Press. [JGP]

Bardunias, P. M. \& Jander, R. (2000) Three dimensional path integration in the house mouse (Mus domestica). Naturwissenschaften 87(12):532-34. [aKJJ]

Barnett-Cowan, M., Dyde, R. T. \& Harris, L. R. (2005) Is an internal model of head orientation necessary for oculomotor control? Annals of the New York Academy of Sciences 1039:314-24. [MB-C]
Barnett-Cowan, M., Fleming, R. W., Singh, M. \& Bülthoff, H. H. (2011) Perceived object stability depends on multisensory estimates of gravity. PLoS ONE 6(4): e19289. [MB-C]

Barnett-Cowan, M., Meilinger, T., Vidal, M., Teufel, H. \& Bülthoff, H. H. (2012) MPI CyberMotion Simulator: Implementation of a novel motion simulator to investigate multisensory path integration in three dimensions. Journal of Visualized Experiments (63): 3436 . [MB-C]

Baron-Cohen, S. (2008) Autism, hypersystemizing, and truth. The Quarterly Journal of Experimental Psychology 61(1):64-75. [KAL]

Barry, C., Hayman, R., Burgess, N. \& Jeffery, K. J. (2007) Experience-dependent rescaling of entorhinal grids. Nature Neuroscience 10(6):682-84. [arKJJ]

Barry, C., Lever, C., Hayman, R., Hartley, T., Burton, S., O’Keefe, J., Jeffery, K. J. \& Burgess, N. (2006) The boundary vector cell model of place cell firing and spatial memory. Reviews in the Neurosciences 17(1-2):71-79. [PAD]

Benedikt, M. L. (1979) To take hold of space: Isovists and isovist fields. Environment and Planning B: Planning and Design 6:47-65. [CH]

Bennett, A. T. (1996) Do animals have cognitive maps? Journal of Experimental Biology 199:219-24. [SZ]

Bennett, D. A. \& Tang, W. (2006) Modelling adaptive, spatially aware, and mobile agents: Elk migration in Yellowstone. International Journal of Geographical Information Science 20(9):1039-66. doi: 10.1080/13658810600830806. [AMH]

Beraldin, J. A., Blais, F. \& Lohr, U. (2010) Laser scanning technology. In: Airborne and terrestrial laser scanning, ed. G. Vosselman \& H. Maas, pp. 1-42. Whittles. [AMH]

Bertamini, M., Lawson, R., Jones, L. \& Winters, M. (2010) The Venus effect in real life and in photographs. Attention, Perception, and Psychophysics 72(7):194864. DOI: 10.3758/APP.72.7.1948. [IB]

Bertamini, M., Spooner, A. \& Hecht, H. (2003) Naïve optics: Predicting and perceiving reflections in mirrors. Journal of Experimental Psychology: Human Perception and Performance 29(5):982-1002. doi: 10.1037/00961523.29.5.982. [IB]

Bertamini, M. \& Wynne, L. (2009) The tendency to overestimate what is visible in a planar mirror amongst adults and children. European Journal of Cognitive Psychology 22:516-28. doi: 10.1080/09541440902934087. [IB]

Bhalla, M. \& Proffitt, D. R. (1999) Visual-motor recalibration in geographical slant perception. Journal of Experimental Psychology: Human Perception and Performance 25(4):1076-96. [aKJJ]

Bianchi, I., Burro, R., Torquati, S. \& Savardi, U. (2013) The middle of the road: Perceiving intermediates. Acta Psychologica 144(1):121-35. DOI: 10.1016/j. actpsy.2013.05.005. [IB]

Bianchi, I. \& Savardi, U. (2008) The relationship perceived between the real body and the mirror image. Perception 5:666-87. [IB]

Bianchi, I. \& Savardi, U. (2012a) The cognitive dimensions of contrariety. In: The square of opposition. A general framework for cognition, ed. J.-Y. Bezieau \& G. Payette, pp. 443-70. Peter Lang. [IB]

Bianchi, I. \& Savardi, U. (2012b) What fits into a mirror: Naïve beliefs on the field of view of mirrors. Journal of Experimental Psychology: Human Perception and Performance 38(5):1144-58. doi: 10.1037/a0027035. [IB]

Bianchi, I., Savardi, U. \& Burro, R. (2011a) Perceptual ratings of opposite spatial properties: Do they lie on the same dimension? Acta Psychologica 138(3):40518. doi: 10.1016/j.actpsy.2011.08.003. [IB]

Bianchi, I., Savardi, U. \& Kubovy, M. (2011b) Dimensions and their poles: A metric and topological theory of opposites. Language and Cognitive Processes 26 (8):1232-65. doi: 10.1080/01690965.2010.520943. [IB]

Blohm, G., Keith, G. P. \& Crawford, J. D. (2009) Decoding the cortical transformations for visually guided reaching in 3D space. Cerebral Cortex 19:1372 93. [SRL]

Blum, K. I. \& Abbott, L. F. (1996) A model of spatial map formation in the hippocampus of the rat. Neural Computation 8:85-93. [FTS]

Boccara, C. N., Sargolini, F., Thoresen, V. H., Solstad, T., Witter, M. P., Moser, E. I. \& Moser, M.-B. (2010) Grid cells in pre- and parasubiculum. Nature Neuroscience 13:987-94. [FSa]

Boyer, W., Longo, M. R. \& Bertenthal, B. I. (2012) Is automatic imitation a specialized form of stimulus-response compatibility? Dissociating imitative and spatial compatibilities. Acta Psychologica 139(3):440-48. doi:10.1016/j. actpsy.2012.01.00. [IB]

Bressan, P., Garlaschelli, L. \& Barracano, M. (2003) Anti-gravity hills are visual illusions. Psychological Science 14:441-49. [HER]

Breveglieri, R., Hadjidimitrakis, K., Bosco, A., Sabatini, S. P., Galletti, C. \& Fattori, P. (2012) Eye position encoding in three-dimensional space: Integration of version and vergence signals in the medial posterior parietal cortex. Journal of Neuroscience 32:159-69. [SRL]

Bridgeman, B. \& Hoover, M. (2008) Processing spatial layout by perception and sensorimotor interaction. The Quarterly Journal of Experimental Psychology 61 (6):851-59. [HER]

Brill, R., Lutcavage, M., Metzger, G., Stallings, J., Bushnell, P., Arendt, M., Lucy, J., Watson, C. \& Foley, D. (2012) Horizontal and vertical movements of juvenile 
References/Jeffery et al.: Navigating in a three-dimensional world

North Atlantic Bluefin Tuna (Thunnus thynnus) in the western North Atlantic determined using ultrasonic telemetry, with reference to population assessment by aerial surveys. Fortschritte der Zoologie 100:155-67. [aKJJ]

Brodsky, M. C., Donahue, S. P., Vaphiades, M. \& Brandt, T. (2006) Skew deviation revisited. Survey of Ophthalmology 51:105-28. [MB-C]

Brown, M. F. \& Lesniak-Karpiak, K. B. (1993) Choice criterion effects in the radialarm maze - Maze-arm incline and brightness. Learning and Motivation 24 (1):23-39. [aKJJ]

Brown, P. \& Levinson, S. C. (1993) "Uphill” and "downhill” in Tzeltal. Journal of Linguistic Anthropology 3(1):46-74. [IB]

Büchner, S., Hölscher, C. \& Strube, G. (2007) Path choice heuristics for navigation related to mental representations of a building. In: Proceedings of the 2nd European Cognitive Science Conference, Delphi, Greece, 23-27 May, 2007, ed. S. Vosniadou, D. Kayser \& A. Protopapas, pp. 504-509. Erlbaum/Taylor \& Francis. [aKJJ, $\mathrm{ABe}, \mathrm{CH}]$

Bueti, D. \& Walsh, V. (2009) The parietal cortex and the representation of time, space, number and other magnitudes. Philosophical Transactions of the Royal Society B 364:1831-40. [ABa]

Buhot, M.-C., Dubayle, C., Malleret, G., Javerzat, S. \& Segu, L. (2001) Exploration, anxiety, and spatial memory in transgenic anophthalmic mice. Behavioral Neuroscience 115:445-67. [AP]

Burgess, N., Barry, C. \& O’Keefe, J. (2007) An oscillatory interference model of grid cell firing. Hippocampus 17:801-12. [CFM]

Burgess, N. \& O'Keefe, J. (1996) Neuronal computations underlying the firing of place cells and their role in navigation. Hippocampus 6:749-62. [FTS]

Burt de Perera, T., de Vos, A. \& Guilford, T. (2005) The vertical component of a fish's spatial map. Animal Behaviour 70:405-409. [aKJJ]

Butler, D. L., Acquino, A. L., Hissong, A. A. \& Scott, P. A. (1993) Wayfinding by newcomers in a complex building. Journal of the Human Factors and Ergonomics Society 35:159-73. [AP]

Butterworth, B. (1999) A head for figures. Science 284:928-29. [ABa]

Calton, J. L. \& Taube, J. S. (2005) Degradation of head direction cell activity during inverted locomotion. Journal of Neuroscience 25(9):2420-28. [aKJJ]

Carbon, C. C. (2007) Autobahn people: Distance estimations between German cities biased by social factors and the autobahn. Lecture Notes in Artificial Science 4387:489-500. [CCC]

Carey, F. G. (1992) Through the thermocline and back again. Oceanus 35:79-85. [aKJJ]

Carlson, L., Hölscher, C., Shipley, T. \& Conroy Dalton, R. (2010) Getting lost in buildings. Current Directions in Psychological Science 19(5):284-89. [CH]

Chan, A. H. S. \& Chan, K. W. L. (2005) Spatial S-R compatibility of visual and auditory signals: Implications for human-machine interface design. Displays 26 (3):109-19. [IB]

Channon, A. J., Crompton, R. H., Günther, M. M., D’Août, K. \& Vereecke, E. E. (2010) The biomechanics of leaping in gibbons. American Journal of Physical Anthropology 143(3):403-16. doi: 10.1002/ajpa.21329. [AMH]

Chebat, D. R., Chen, J. K., Schneider, F., Ptito, A., Kupers, R. \& Ptito, M. (2007) Alterations in right posterior hippocampus in early blind individuals. NeuroReport 18:329-33. [AP]

Chen, A., DeAngelis, G. C. \& Angelaki, D. E. (2010) Macaque parieto-insular vestibular cortex: Responses to self-motion and optic flow. Journal of Neuroscience 30(8):3022-42. [GAO]

Chen, A., DeAngelis, G. C. \& Angelaki, D. E. (2011a) Convergence of vestibular and visual self-motion signals in an area of the posterior sylvian fissure. Journal of Neuroscience 31(32):11617-27. [GAO]

Chen, A., DeAngelis, G. C. \& Angelaki, D. E. (2011b) Representation of vestibular and visual cues to self-motion in ventral intraparietal cortex. Journal of Neuroscience 31(33):12036-252. [GAO]

Chittka, L. \& Jensen, K. (2011) Animal cognition: Concepts from apes to bees. Current Biology 21:R116-19. [AGD]

Chittka, L. \& Niven, J. (2009) Are bigger brains better? Current Biology 19:R9951008. [AGD]

Clark, H. H. (1973) Space, time, semantics, and the child. In: Cognitive development and the acquisition of language, ed. T. E. Moore, pp. 27-63. Academic Press. $[\mathrm{KJH}]$

Cockburn, A. \& McKenzie, B. (2001) 3D or not 3D? Evaluating the effect of the third dimension in a document management system. In: ed. M. BeaudouinLafon \& R. J. K. Jacob, Proceedings of CHI'2001, Conference on Human Factors in Computing Systems, pp. 434-41. ACM Press. [DMWP]

Cockburn, A. \& McKenzie, B. (2002) Evaluating the effectiveness of spatial memory in $2 \mathrm{D}$ and $3 \mathrm{D}$ physical and virtual environments. In: ed. L. Terveen, Proceedings of CHI'2002, Conference on Human Factors in Computing Systems, pp. 203-10. ACM Press. [DMWP]

Cohen, R. G. \& Rosenbaum, D. A. (2004) Where objects are grasped reveals how grasps are planned: Generation and recall of motor plans. Experimental Brain Research 157:486-95. [ABa]

Collins, A. M. \& Quillian, M. R. (1969) Retrieval time from semantic memory. Journal of Verbal Learning and Verbal Behavior 8:240-47. [HS]
Conroy Dalton, R. (2003) The secret is to follow your nose: Route path selection an angularity. Environment and Behavior 35(1):107-31. [CH]

Conroy Dalton, R. (2005) Space syntax and spatial cognition. World Architecture 185:41-47, 107-111. [CH]

Corballis, M. C. (2013) Mental time travel: A case for evolutionary continuity. Trends in Cognitive Sciences 17:5-6. [ABa]

Cornelis, E. V., van Doorn, A. J. \& Wagemans, J. (2009) The effects of mirror reflections and planar rotations of pictures on the shape percept of the depicted object. Perception 38(10):1439-66. [IB]

Creem, S. H. \& Proffitt, D. R. (1998) Two memories for geographical slant: Separation and interdependence of action and awareness. Psychonomic Bulletin and Review 5(1):22-36. [aKJJ]

Creem, S. H. \& Proffitt, D. R. (2001) Defining the cortical visual systems: "What," "where," and "how." Acta Psychologica (Amsterdam) 107(1-3):43-68. [aKJJ]

Creem-Regehr, S. H., Mohler, B. J. \& Thompson, W. B. (2004) Perceived slant is greater from far versus near distances. (Abstract). Journal of Vision 4 (8):374a. doi:10.1167/4.8.374. Available at: http://journalofvision.org/4/8/ 374/ [HER]

Croucher, C. J., Bertamini, M. \& Hecht, H. (2002) Naïve optics: Understanding the geometry of mirror reflections. Journal of Experimental Psychology: Human Perception and Performance 28:546-62. doi: 10.1037/0096-1523.28.3.546. [IB]

Cruse, D. A. (1986) Lexical semantics. (Cambridge Textbooks in Linguistics). Cambridge University Press. [IB]

Cutting, J. M. \& Vishton, P. M. (1995) Perceiving layout and knowing distances: The integration, relative potency, and contextual use of different information about depth. In: Handbook of perception and cognition, vol. 5: Perception of space and motion, ed. W. Epstein \& S. Rogers, pp. 69-117. Academic Press. [RLK]

Dacke, M. \& Srinivasan, M. V. (2007) Honeybee navigation: Distance estimation in the third dimension. Journal of Experimental Biology 210(Pt. 5):845-53. [aKJJ, AGD]

Dalrymple, K. A. \& Kingstone, A. (2010) Time to act and attend to the real mechanisms of action and attention. British Journal of Psychology 101:213-16. [KAL]

Dehaene, S., Bossini, S. \& Giraux, P. (1993) The mental representation of parity and number magnitude. Journal of Experimental Psychology: General 122:37196. $[\mathrm{ABa}]$

Derdikman, D. \& Moser, E. I. (2010) A manifold of spatial maps in the brain. Trends in Cognitive Sciences 14(12):561-69. [HY]

Derdikman, D., Whitlock, J. R., Tsao, A., Fyhn, M., Hafting, T., Moser, M. \& Moser, E. I. (2009) Fragmentation of grid cell maps in a multicompartment environment. Nature Neuroscience 12(10):1325-32. [PAD, FSa, HY]

Deutschländer, A., Stephan, T., Hüfner, K., Wagner, J., Wiesmann, M., Strupp, M., Brandt, T. \& Jahn, K. (2009) Imagined locomotion in the blind: An fMRI study. NeuroImage 45:122-28. [AP]

Di Fiore, A. \& Suarez, S. A. (2007) Route-based travel and shared routes in sympatric spider and woolly monkeys: Cognitive and evolutionary implications. Animal Cognition 10(3):317-29. doi: 10.1007/s10071-006-0067-y. [AMH]

Domjan, M. (2009) The principles of learning and behavior, 6th edition. Wadsworth, Cengage Learning. [TBdP]

Dudchenko, P. A. \& Zinyuk, L. E. (2005) The formation of cognitive maps of adjacent environments: Evidence from the head direction cell system. Behavioral Neuroscience 119(6):1511-23. [aKJJ, PAD]

Durgin, F. H., Hajnal, A., Li, Z., Tonge, N. \& Stigliani, A. (2010a) Palm boards are not action measures: An alternative to the two-systems theory of geographical slant perception. Acta Psychologica 134:182-97. [FHD]

Durgin, F. H., Hajnal, A., Li, Z., Tonge, N. \& Stigliani, A. (2011) An imputed dissociation might be an artifact: Further evidence for the generalizability of the observations of Durgin et al. 2010. Acta Psychologica (Amsterdam) 138(2):28184. [aKJJ]

Durgin, F. H., Klein, B., Spiegel, A., Strawser, C. J. \& Williams, M. (2012) The social psychology of perception experiments: Hills, backpacks, glucose and the problem of generalizability. Journal of Experimental Psychology: Human Perception and Performance 2012;38(6):1582-95. [aKJJ]

Durgin, F. H. \& Li, Z. (2011) Perceptual scale expansion: An efficient angular coding strategy for locomotor space. Attention, Perception, and Psychophysics 73 (6):1856-70. [aKJJ, FHD]

Durgin, F. H. \& Li, Z. (2012) Spatial biases and the haptic experience of surface orientation. In: Haptics rendering and applications, ed. A. El Saddik, pp. 75-94. Intech. [FHD]

Durgin, F. H., Li, Z. \& Hajnal, A. (2010b) Slant perception in near space is categorically biased: Evidence for a vertical tendency. Attention, Perception, and Psychophysics 72:1875-89. [FHD]

Dyde, R. T., Jenkin, M. R. \& Harris, L. R. (2006) The subjective visual vertical and the perceptual upright. Experimental Brain Research 173:612-22. [MB-C]

Dyer, A. G., Rosa, M. G. P. \& Reser, D. H. (2008) Honeybees can recognise images of complex natural scenes for use as potential landmarks. The Journal of Experimental Biology 211:1180-86. [AGD] 
References/Jeffery et al.: Navigating in a three-dimensional world

Dyer, F. C. (1991) Bees acquire route-based memories but not cognitive maps in a familiar landscape. Animal Behaviour 41:239-46. [SZ]

Eaton, M. D., Evans, D. L., Hodgson, D. R. \& Rose, R. J. (1995) Effect of treadmill incline and speed on metabolic rate during exercise in thoroughbred horses. Journal of Applied Physiology 79(3):951-57. [FTS]

Eckles, M. A., Roubik, D. W. \& Nieh, J. C. (2012) A stingless bee can use visual odometry to estimate both height and distance. The Journal of Experimental Biology 215:3155-60. [AGD]

Epstein, R. A. (2008) Parahippocampal and retrosplenial contributions to human spatial navigation. Trends in Cognitive Sciences 12:388-96. [SRL]

Esch, H. E. \& Burns, J. E. (1995) Honeybees use optic flow to measure the distance of a food source. Naturwissenschaften 82:38-40. [aKJJ]

Etienne, A. S. \& Jeffery, K. J. (2004) Path integration in mammals. Hippocampus 14:180-92. [aKJJ]

European Aviation Safety Agency. (2006) EASA Annual Safety Review, 2006. EASA (European Aviation Safety Agency, Cologne, Germany). [MB-C]

Euston, D. R. \& Takahashi, T. T. (2002) From spectrum to space: The contribution of level difference cues to spatial receptive fields in the barn owl inferior colliculus. Journal of Neuroscience 22(1):284-93. [FTS]

Eyferth, K., Niessen, C. \& Spaeth, O. (2003) A model of air traffic controllers' conflict detection and conflict resolution. Aerospace Science and Technology 7:409-16. [HS]

Fenton, A. A., Kao, H. Y., Neymotin, S. A., Olypher, A., Vayntrub, Y., Lytton, W. W. \& Ludvig, N. (2008) Unmasking the CAl ensemble place code by exposures to small and large environments: More place cells and multiple, irregularly arranged, and expanded place fields in the larger space. Journal of Neuroscience 28(44):11250-62. [aKJJ]

Finkelstein, A., Derdikman, D., Foerster, J., Las, L. \& Ulanovsky, N. (2012) 3-D head-direction cells in the bat presubiculum. Society for Neuroscience Abstracts, Abstract No. 38:203.19. [FSt]

Foley, H. J. \& Matlin, M. W. (2010) Sensation and perception, 5th edition. Allyn and Bacon. [TBdP]

Foley, J. M., Ribeiro, N. P. \& Da Silva, J. (2004) Visual perception of extent and the geometry of visual space. Vision Research 44:147-56. [FHD]

Foo, P., Warren, W. H., Duchon, A. \& Tarr, M. J. (2005) Do humans integrate routes into a cognitive map? Map- versus landmark-based navigation of novel shortcuts. Journal of Experimental Psychology: Learning, Memory, and Cognition 31(2):195-215. [CCC]

Forbus, K. D. (2011) Qualitative modeling. WIREs Cognitive Science 2:374-91. [HS]

Foulsham, T. \& Kingstone, A. (2012) Goal-driven and bottom-up gaze in an active real-world search task. In: Proceedings of the Symposium on Eye-Tracking Research and Applications (ETRA '12), Santa Barbara, CA, March 28-30, 2012, ed. C. H. Morimoto, H. O. Istance, J. B. Mulligan, P. Qvarfordt \& S. N. Spencer, pp. 189-92. ACM Digital Library. [KAL]

Foulsham, T., Walker, E. \& Kingstone, A. (2011) The where, what and when of gaze allocation in the lab and the natural environment. Vision Research 51(17):192031. [KAL]

Frank, L. M., Brown, E. N. \& Wilson, M. (2000) Trajectory encoding in the hippocampus and entorhinal cortex. Neuron 27:169-78. [HY]

Frankenstein, J., Büchner, S. J., Tenbrink, T. \& Hölscher, C. (2010) Influence of geometry and objects on local route choices during wayfinding. In: Spatial Cognition VII: Proceedings of the Seventh International Conference on Spatial Cognition, Mt. Hood/Portland, OR, USA, August 15-19, 2010, ed. C. Hölscher, T. F. Shipley, M. O. Belardinelli, J. A. Bateman \& N. S. Newcombe, pp. 41-53. Springer. $[\mathrm{CH}]$

Frankenstein, J., Mohler, B. J., Bülthoff, H. H. \& Meilinger, T. (2012) Is the map in our head oriented north? Psychological Science 23:120-25. [AP]

Franklin, N. \& Tversky, B. (1990) Searching imagined environments. Journal of Experimental Psychology: General 119:63-76. [KJH]

Fuhs, M. C., VanRhoads, S. R., Casale, A. E., McNaughton, B. L. \& Touretzky, D. S. (2005) Influence of path integration versus environmental orientation on place cell remapping between visually identical environments. Journal of Neurophysiology 94:2603-16. [PAD]

Gagliaro, A., Ioalè, P. \& Bingman, V. P. (1999) Homing in pigeons: The role of the hippocampal formation in the representation of landmarks used for navigation. Journal of Neuroscience 19:311-15. [DN]

Galati, G., Pelle, G., Berthoz, A. \& Committeri, G. (2010) Multiple reference frames used by the human brain for spatial perception and memory. Experimental Brain Research 206(2):109-20. [rKJJ]

Garling, T., Anders, B., Lindberg, E. \& Arce, C. (1990) Is elevation encoded in cognitive maps? Journal of Environmental Psychology 10:341-51. [aKJJ]

Georges-François, P., Rolls, E. T. \& Robertson, R. G. (1999) Spatial view cells in the primate hippocampus: Allocentric view not head direction or eye position or place. Cerebral Cortex 9:197-212. [SRL]

Gevers, W., Lammertyn, J., Notebaert, W., Verguts, T. \& Fias, W. (2006) Automatic response activation of implicit spatial information: Evidence from the SNARC effect. Acta Psychologica 122:221-33. [ABa]
Gibson, J. J. (1979) The ecological approach to visual perception. Houghton Mifflin. [CCC, FHD]

Gibson, J. J. \& Cornsweet, J. (1952) The perceived slant of visual surfaces - Optical and geographical. Journal of Experimental Psychology 44(1):11-15. [aKJJ]

Gilbert, A. L., Regier, T., Kay, P. \& Ivry, R. B. (2006) Whorf hypothesis is supported in the right visual field but not the left. Proceedings of the National Academy of Sciences USA 103:489-94. [KJH]

Giudice, N. A., Klatzky, R. L., Bennett, C. \& Loomis, J. M. (2013) Perception of 3-D location based on vision, touch, and extended touch. Experimental Brain Research 224:141-53. [RLK]

Goodale, M. A. \& Milner, A. D. (1992) Separate visual pathways for perception and action. Trends in Neurosciences 15:20-25. [SRL]

Goodridge, J. P., Dudchenko, P. A., Worboys, K. A., Golob, E. J. \& Taube, J. S. (1998) Cue control and head direction cells. Behavioral Neuroscience 112 (4):749-61. [aKJJ]

Grah, G., Wehner, R. \& Ronacher, B. (2007) Desert ants do not acquire and use a three-dimensional global vector. Frontiers in Zoology 4:12. [aKJJ, RFW]

Gregory, E. \& McCloskey, M. (2010) Mirror-image confusions: Implications for representation and processing of object orientation. Cognition 116(1):110-29. doi: 10.1016/j.cognition.2010.04.005. [IB]

Grieves, R. M. \& Dudchenko, P. A. (2013) Cognitive maps and spatial inference in animals: Rats fail to take a novel shortcut, but can take a previously experienced one. Learning and Motivation 44:81-92. [PAD]

Griffin, D. R. (1958) Listening in the dark. Yale University Press. [CFM]

Grobéty, M. C. \& Schenk, F. (1992a) Spatial learning in a three-dimensional maze. Animal Behaviour 43(6):1011-20. [aKJJ, TBdP, DN]

Grobéty, M.-C. \& Schenk, F. (1992b) The influence of spatial irregularity upon radial-maze performance in the rat. Animal Learning and Behavior 20(4):393400. [aKJ]

Grush, R. (2007) Skill theory v2.0: Dispositions, emulation, and spatial perception. Synthese 159(3):389-416. [DMK]

Gu, Y., Fetsch, C. R., Adeyemo, B., DeAngelis, G. C. \& Angelaki, D. E. (2010) Decoding of MSTd population activity accounts for variations in the precision of heading perception. Neuron 66(4):596-609. [GAO]

Hadjidimitrakis, K., Breveglieri, R., Bosco, A. \& Fattori, P. (2012) Three-dimensional eye position signals shape both peripersonal space and arm movement activity in the medial posterior parietal cortex. Frontiers in Integratve Neuroscience 6:37. doi: 10.3389/fnint.2012.00037. [SRL]

Hafting, T., Fyhn, M., Molden, S., Moser, M. B. \& Moser, E. I. (2005) Microstructure of a spatial map in the entorhinal cortex. Nature 436:801-806. [aKJJ]

Hajnal, A., Abdul-Malak, D. T. \& Durgin, F. H. (2011) The perceptual experience of slope by foot and by finger. Journal of Experimental Psychology: Human Perception and Performance 37:709-19. [FHD]

Hartley, T., Burgess, N., Lever, C., Cacucci, F. \& O’Keefe, J. (2000) Modelling place fields in terms of the cortical inputs to the hippocampus. Hippocampus 10:36979. [PAD]

Harvey, P. H. \& Pagel, M. D. (1991) The comparative method in evolutionary biology. Oxford University Press. [DN]

Hasselmo, M. E., Giocomo, L. M. \& Zilli, E. A. (2007) Grid cell firing may arise from interference of theta frequency membrane potential oscillations in single neurons. Hippocampus 17(12):1252-71. [CFM]

Hayman, R., Verriotis, M. A., Jovalekic, A., Fenton, A. A. \& Jeffery, K. J. (2011) Anisotropic encoding of three-dimensional space by place cells and grid cells. Nature Neuroscience 14(9):1182-88. [arKJJ, MB-C, TBdP, PAD, DMK, FSa, HY]

Henderson, J., Hurly, T. A. \& Healy, S. D. (2001) Rufous hummingbirds' memory for flower location. Animal Behaviour 61(5):981-86. [aKJJ]

Henderson, J., Hurly, T. A. \& Healy, S. D. (2006) Spatial relational learning in rufous hummingbirds (Selasphorus rufus). Animal Cognition 9:201-205. $[\mathrm{aKJJ}]$

Hengstenberg, R. (1991) Gaze control in the blowfly Calliphora: A multisensory, two-stage integration process. Seminars in Neuroscience 3(1):19-29. Available at: http://www.sciencedirect.com/science/article/pii/104457659190063T [MB-C]

Hess, D., Koch, J. \& Ronacher, B. (2009) Desert ants do not rely on sky compass information for the perception of inclined path segments. Journal of Experimental Biology 212(10):1528-34. [aKJJ]

Heys, J.G., MacLeod, K., Moss, C.F., and Hasselmo, M. (2013) Bat and rat neurons differ in theta frequency resonance despite similar spatial coding. Science 340:363-67. [CFM]

Higashiyama, A. \& Ueyama, E. (1988) The perception of vertical and horizontal distances in outdoor settings. Perception and Psychophysics 44:151-56. doi: 10.3758/BF03208707. [FHD]

Hill, A. J. \& Best, P. J. (1981) Effects of deafness and blindness on the spatial correlates of hippocampal unit activity in the rat. Experimental Neurology 74:20417. $[\mathrm{AP}]$

Hill, C. (1982) Up/down, front/back, left/right: A contrastive study of Hausa and English. In: Here and there: Crosslinguistic studies on deixis and demonstration, ed. J. Weissenborn \& W. Klein, pp. 11-42. John Benjamins. [IB] 
References/Jeffery et al.: Navigating in a three-dimensional world

Hillier, B. \& Hanson, J., eds. (1984) The social logic of space. Cambridge University Press. [CH]

Holbrook, R. I. \& Burt de Perera, T. B. (2009) Separate encoding of vertical and horizontal components of space during orientation in fish. Animal Behaviour 78 (2):241-45. [TBdP, CCC, aKJ]]

Holbrook, R. I. \& Burt de Perera, T. (2011a) Fish navigation in the vertical dimension: Can fish use hydrostatic pressure to determine depth? Fish and Fisheries 12(4):370-379. [aKJJ]

Holbrook, R. I. \& Burt de Perera, T. (2011b) Three-dimensional spatial cognition: Information in the vertical dimension overrides information from the horizontal. Animal Cognition 14:613-19. [TBdP]

Holmes, K. J. (2012) Language as a window into the mind: The case of space. Doctoral dissertation, Department of Psychology, Emory University. [KJH]

Holmes, K. J. \& Wolff, P. (2012) Does categorical perception in the left hemisphere depend on language? Journal of Experimental Psychology: General 141:43943. [KJH]

Holmes, K. J. \& Wolff, P. (2013a) Spatial language and the psychological reality of schematization. Cognitive Processing 14:205-208. [KJH]

Holmes, K. J. \& Wolff, P. (2013b) When is language a window into the mind? Looking beyond words to infer conceptual categories. In: Proceedings of the 35 th annual conference of the Cognitive Science Society, eds. M. Knauff, M. Pauen, N. Sebanz \& I. Wachsmuth, pp. 597-602. Cognitive Science Society. $[\mathrm{KJH}]$

Hölscher, C., Büchner, S., Brösamle, M., Meilinger, T. \& Strube, G. (2007) Signs and maps - Cognitive economy in the use of external aids for indoor navigation. In: Proceedings of the 29th Annual Cognitive Science Society, ed. D. S. McNamara \& J. G. Trafton, pp. 377-82. Cognitive Science Society. [CH]

Hölscher, C., Büchner, S., Meilinger, T. \& Strube, G. (2009) Adaptivity of wayfinding strategies in a multi-building ensemble: The effects of spatial structure, task requirements, and metric information. Journal of Environmental Psychology 29(2):208-19. [CH]

Hölscher, C., Brösamle, M. \& Vrachliotis, G. (2012) Challenges in multi-level wayfinding: A case-study with space syntax technique. Environment and Planning B: Planning and Design 39:63-82. [CH]

Hölscher, C., Meilinger, T., Vrachliotis, G., Brösamle, M. \& Knauff, M. (2006) Up the down staircase: Wayfinding strategies and multi-level buildings. Journal of Environmental Psychology 26(4):284-99. [aKJJ, ABe, CCC, CH, AP]

Hommel, B., Müsseler, J., Aschersleben, G. \& Prinz, W. (2001) The theory of event coding (TEC): A framework for perception and action planning. Behavioral and Brain Sciences 24:849-78. [ABa]

Howard, A., Bernardes, S., Nibbelink, N., Biondi, L., Presotto, A., Fragaszy, D. \& Madden, M. (2012) A maximum entropy model of the bearded capuchin monkey habitat incorporating topography and spectral unmixing analysis. Annals of the International Society of Photogrammetry and Remote Sensing 1 (2):7-11. [AMH]

Hubbard, E. M., Piazza, M., Pinel, P. \& Dehaene, S. (2005) Interactions between number and space in parietal cortex. Nature Reviews Neuroscience 6:435-48. [ABa]

Huttenlocher, J., Hedges, L. V. \& Duncan, S. (1991) Categories and particulars: Prototype effects in estimating spatial location. Psychological Review 98:352 76. $[\mathrm{RFW}]$

Hyvärinen, J., Hyvärinen, L. \& Linnankoski, I. (1981) Modification of parietal association cortex and functional blindness after binocular deprivation in young monkeys. Experimental Brain Research 42:1-8. [AP]

Indovina, I., Maffei, V., Pauwels, K., Macaluso, E., Orban, G. A. \& Lacquaniti, F. (2013 Simulated self-motion in a visual gravity field: Brain sensitivity to vertical and horizontal heading in the human brain. NeuroImage 71C:114-24. [GAO]

Ingram, J. N. \& Wolpert, D. M. (2011) Naturalistic approaches to sensorimotor control. Progress in Brain Research 191:3-29. [KAL]

Jacobs, J., Kahana, M. J., Ekstrom, A. D., Mollison, M. V. \& Fried, I. (2010) A sense of direction in human entorhinal cortex. Proceedings of the National Academy of Sciences USA 107(14):6487-92. [GAO]

Janson, C. H. (1998) Experimental evidence for spatial memory in foraging wild capuchin monkeys, Cebus apella. Animal Behaviour 55(5):1229-43. doi: 10.1006/anbe.1997.0688. [AMH]

Janson, C. H. (2007) Experimental evidence for route integration and strategic planning in wild capuchin monkeys. Animal Cognition 10(3):341-56. doi:10.1007/s10071-007-0079-2. [AMH]

Jeffery, K. J. (2007) Integration of the sensory inputs to place cells: What, where, why, and how? Hippocampus 17(9):775-85. [aKJJ]

Jeffery, K. J., Anand, R. L. \& Anderson, M. I. (2006) A role for terrain slope in orienting hippocampal place fields. Experimental Brain Research 169(2):21825. [aKJJ]

Jeffery, K. J. \& Burgess, N. (2006) A metric for the cognitive map - Found at last? Trends in Cognitive Sciences 10(1):1-3. [aKJJ]

Jensen, M. E., Moss, C. F. \& Surlykke, A. (2005) Echolocating bats and their use of landmarks and spatial memory. Journal of Experimental Biology 208:4399410. [CFM]
Jovalekic, A., Hayman, R., Becares, N., Reid, H., Thomas, G., Wilson, J. \& Jeffery, K. (2011) Horizontal biases in rats' use of three-dimensional space. Behavioural Brain Research 222(2):279-88. [aKJJ, TBdP, DN, RFW]

Judd, S. \& Collett, T. (1998) Multiple stored views and landmark guidance in ants. Nature 392:710-14. [SZ]

Kamil, A. C., Balda, R. P. \& Olson, D. J. (1994) Performance of four seed-caching corvid species in the radial arm-maze analog. Journal of Comparative Psychology 108:385-93. [DN]

Kammann, R. (1967) The overestimation of vertical distance and its role in the moon illusion. Attention, Perception, and Psychophysics 2(12):585-89. [aKJJ]

Kelly, J. (2011) Head for the hills: The influence of environmental slant on spatial memory organization. Psychonomic Bulletin and Review 18(4):774-80. [aKJJ]

Kelly, J. W., Loomis, J. M. \& Beall, A. C. (2004) Judgments of exocentric direction in large-scale space. Perception 33:443-54. [FHD]

Killian, N. J., Jutras, M. J. \& Buffalo, E. A. (2012) A map of visual space in the primate entorhinal cortex. Nature 491(7426):761-64. [GAO]

Klatzky, R. L. (1998) Allocentric and egocentric spatial representations: Definitions, distinctions, and interconnections. In: Spatial cognition - An interdisciplinary approach to representation and processing of spatial knowledge (Lecture Notes in Artificial Intelligence, vol. 1404), ed. C. Freksa, C. Habel \& K. F. Wender, pp. 1-17. Springer-Verlag. [RLK]

Kluzik, J., Horak, F. B. \& Peterka, R. J. (2005) Differences in preferred reference frames for postural orientation shown by after-effects of stance on an inclined surface. Experimental Brain Research 162:474-89. Available at: http://ink. springer.com/article/10.1007\%2Fs00221-004-2124-6 [MB-C]

Knauff, M., Rauh, R. \& Renz, J. (1997) A cognitive assessment of topological spatial relations: Results from an empirical investigation. In: Proceedings of the $3 \mathrm{rd}$ International Conference on Spatial Information Theory (COSIT'97), ed. S. C. Hirtle \& A. U. Frank, pp. 193-206. Springer. [HS]

Knierim, J. J. \& Hamilton, D. A. (2011) Framing spatial cognition: Neural representations of proximal and distal frames of reference and their roles in navigation. Physiological Reviews 91:1245-79. [FSa]

Knierim, J. J. \& McNaughton, B. L. (2001) Hippocampal place-cell firing during movement in three-dimensional space. Journal of Neurophysiology 85(1):10516. $[\mathrm{aKJJ}]$

Knierim, J. J., McNaughton, B. L. \& Poe, G. R. (2000) Three-dimensional spatial selectivity of hippocampal neurons during space flight. Nature Neuroscience 3 (3):209-10. [aKJJ, DMK]

Knierim, J. J., Poe, G. R. \& McNaughton, B. L. (2003) Ensemble neural coding of place in zero-g. In: The Neurolab Spacelab Mission: Neuroscience research in space. Results from the STS-90, Neurolab Spacelab Mission, NASA Report SP2003-535, ed. J. C. Buckey, Jr. \& J. L. Homick, pp. 63-68. NASA. [aKJJ]

Knierim, J. J. \& Rao, G. (2003) Distal landmarks and hippocampal place cells: Effects of relative translation versus rotation. Hippocampus 13:604-17. [FSa]

Knudsen, E. I. \& Konishi, M. (1978a) A neural map of auditory space in the owl. Science 200:795-97. [FTS]

Knudsen, E. I. \& Konishi, M. (1978b) Space and frequency are represented separately in auditory midbrain of the owl. Journal of Neurophysiology 41:870-84. [FTS]

Konishi, M. (1973) How the owl tracks its prey. American Science 61:414-24. [FTS]

Kosslyn, S. M., Koenig, O., Barrett, A., Cave, C. B., Tang, J. \& Gabrieli, J. D. E. (1989) Evidence for two types of spatial representations: Hemispheric specialization for categorical and coordinate relations. Journal of Experimental Psychology: Human Perception and Performance 15:723-35. [KJH]

Kravitz, D. J., Saleem, K. S., Baker, C. I. \& Mishkin, M. (2011) A new neural framework for visuospatial processing. Nature Reviews Neuroscience 12(4):21730. [SRL, GAO]

Kravitz, D. J., Saleem, K. S., Baker, C. I., Ungerleider, L. G. \& Mishkin, M. (2013) The ventral visual pathway: An expanded neural framework for the processing of object quality. Trends in Cognitive Sciences 17(1):26-49. [GAO]

Krogh, A. (1929) The progress of physiology. American Journal of Physiology 90:243-51. [CFM]

Kropff, E. \& Treves, A. (2008) The emergence of grid cells: Intelligent design or just adaptation? Hippocampus 18(12):1256-69. doi: 10.1002/hipo.20520. [FSt]

Krumnack, A., Bucher, L., Nejasmic, J., Nebel, B. \& Knauff, M. (2011) A model for relational reasoning as verbal reasoning. Cognitive Systems Research 11:37792. [HS]

Kubovy, M. (2002) Phenomenology, psychological. In: Encyclopedia of cognitive science, ed. L. Nadel, pp. 579-86. Nature Publishing Group. [IB]

Kubovy, M. \& Gepshtein, S. (2003) Grouping in space and in space-time: An exercise in phenomenological psychophysics. In: Perceptual organization in vision: Behavioral and neural perspectives, ed. R. Kimchi, M. Behrmann \& C. R. Olson, pp. 45-86. Erlbaum. [IB]

Kuipers, B., Browning, R., Gribble, B., Hewett, M. \& Remolina, E. (2000) The spatial semantic hierarchy. Artificial Intelligence 119:191-233. [HP]

Kupers, R., Chebat, D. R., Madsen, K. H., Paulson, O. B. \& Ptito, M. (2010) Neural correlates of virtual route recognition in congenital blindness. Proceedings of the National Academy of Sciences USA 107:12716-21. [AP] 
References/Jeffery et al.: Navigating in a three-dimensional world

Lackner, J. R. \& Graybiel, A. (1983) Perceived orientation in free-fall depends on visual, postural, and architectural factors. Aviation, Space, and Environmental Medicine 54(1):47-51. [aKJJ]

Lagae, L., Maes, H., Raiguel, S., Xiao, D. K. \& Orban, G. A. (1994) Responses of monkey STS neurons to optic flow components: A comparison of areas MT and MST. Journal of Neurophysiology 71(5):1597-626. [GAO]

Lahav, O. \& Mioduser, D. (2008) Haptic-feedback support for cognitive mapping of unknown spaces by people who are blind. International Journal of HumanComputer Studies 66:23-35. [AP]

Landau, B. \& Jackendoff, R. (1993) "What" and "where" in spatial language and spatial cognition. Behavioral and Brain Sciences 16:217-65. [KJH]

Langston, R. F., Ainge, J. A., Couey, J. J., Canto, C. B., Bjerknes, T. L., Witter, M. P., Moser, E. I. \& Moser, M.-B. (2010) Development of the spatial representation system in the rat. Science 328(5985):1576-80. [rKJJ]

Lappe, M., Bremmer, F. \& van den Berg, A. V. (1999) Perception of self-motion from visual flow. Trends in Cognitive Sciences 3(9):329-36. [RFW]

Larsen, D. D., Luu, J. D., Burns, M. E. \& Krubitzer, L. (2009) What are the effects of severe visual impairment on the cortical organization and connectivity of primary visual cortex? Frontiers in Neuroanatomy 3:30. [AP]

Lavie, N. (2005) Distracted and confused? Selective attention under load. Trends in Cognitive Sciences 9(2):75-82. [rKJJ]

Lehky, S. R. \& Sereno, A. B. (2011) Population coding of visual space: Modeling. Frontiers in Computational Neuroscience 4:155. doi:110.3389/ fncom.2010.00155. [SRL]

Lehrer, M. (1994) Spatial vision in the honeybee: The use of different cues in different tasks. Vision Research 34(18):2363-85. [CCC]

Lent, D. D., Graham, P. \& Collett, T. S. (2010) Image-matching during ant navigation occurs through saccade-like body turns controlled by learned visual features. Proceedings of the National Academy of Sciences USA 107:1634853. $[\mathrm{TBdP}]$

Leporé, N., Shi, Y., Lepore, F., Fortin, M., Voss, P., Chou, Y. Y. Lord, C., Lassonde, M., Dinov, I. D., Toga, A. W. \& Thompson, P. M. (2009) Patterns of hippocampal shape and volume differences in blind subjects. NeuroImage 46:949. [AP]

Leutgeb, S., Leutgeb, J. K., Treves, A., Moser, M. B. \& Moser, E. I. (2004) Distinct ensemble codes in hippocampal areas CA3 and CA1. Science 305(5688):129598. [aKJJ]

Lever, C., Burton, S., Jeewajee, A., O’Keefe, J. \& Burgess, N. (2009) Boundary vector cells in the subiculum of the hippocampal formation. Journal of Neuroscience 29:9771-77. [FSa]

Li, Z. \& Durgin, F. H. (2009) Downhill slopes look shallower from the edge. Journal of Vision 9(11):6-15. [aKJJ, HER]

Li, Z. \& Durgin, F. H. (2010) Perceived slant of binocularly viewed large-scale surfaces: A common model from explicit and implicit measures. Journal of Vision 10(14):article 13:1-16. [aKJJ, FHD]

Li, Z. \& Durgin, F. H. (2012) A comparison of two theories of perceived distance on the ground plane: The angular expansion hypothesis and the intrinsic bias hypothesis. i-Perception 3:368-383. [FHD]

Li, Z, Phillips, J. \& Durgin, F. H. (2011) The underestimation of egocentric distance: Evidence from frontal matching tasks. Attention, Perception, and Psychophysics 73:2205-2217. [FHD]

Li, Z., Sun, E., Strawser, C. J., Spiegel, A., Klein, B. \& Durgin, F. H. (2013) On the anisotropy of perceived ground extents and the interpretation of walked distance as a measure of perception. Journal of Experimental Psychology: Human Perception and Performance 39:477-493. [FHD]

Liang, J. C., Wagner, A. D. \& Preston, A. R. (2013) Content representation in the human medial temporal lobe. Cerebral Cortex 23:80-96. [GAO]

Liu, Y., Vogels, R. \& Orban, G. A. (2004) Convergence of depth from texture and depth from disparity in macaque inferior temporal cortex. Journal of Neuroscience $24(15): 3795-800 . \quad$ [GAO]

Loetscher, T., Bockisch, C. J., Nicholls, M. E. R. \& Brugger, P. (2010) Eye position predicts what number you have in mind. Current Biology 20:264-65. [ABa]

Longstaffe, K. A., Hood, B. M. \& Gilchrist, I. D. (2012) Inhibition, attention and working memory in large scale search. Poster presented at the 5th International Conference on Spatial Cognition, "Space and Embodied Cognition," Rome, Italy, September 4-8, 2012. [KAL]

Loomis, J. L., Klatzky, R. L. \& Giudice, N. A. (2013) Representing 3D space in working memory: Spatial images from vision, hearing, touch, and language. In: Multisensory imagery: Theory and applications, ed. S. Lacey \& R. Lawson, pp. 131-55. Springer. [RLK]

Loomis, J. M., Da Silva, J. A., Fujita, N. \& Fukusima, S. S. (1992) Visual space perception and visually directed action. Journal of Experimental Psychology: Human Perception and Performance 18(4):906-921. [aKJJ, FHD]

Loomis, J. M., Klatzky, R. L., Golledge, R. G., Cicinelli, J. G., Pellegrino, J. W. \& Fry, P. A. (1993) Nonvisual navigation by blind and sighted: Assessment of path integration ability. Journal of Experimental Psychology: General 122:73-91. $[\mathrm{MB}-\mathrm{C}, \mathrm{RFW}]$
Loomis, J. M., Lippa, Y., Klatzky, R. L. \& Golledge, R. G. (2002) Spatial updating of locations specified by 3-D sound and spatial language. Journal of Experimental Psychology: Human Learning, Memory, and Cognition 28:335-45. [RLK $]$

Loomis, J. M. \& Philbeck, J. W. (1999) Is the anisotropy of perceived 3-D shape invariant across scale? Perception and Psychophysics 61:397-402. [FHD]

Maas, H. (2010) Forestry applications. In: Airborne and terrestrial laser scanning, ed. G. Vosselman \& H. Maas, pp. 213-35. Whittles. [AMH]

MacEachren, A. M. (1986) A linear view of the world: Strip maps as a unique form of cartographic representation. American Cartographer 13:7-25. [HS]

MacNeilage, P. R., Banks, M. S., Berger, D. R. \& Bülthoff, H. H. (2007) A Bayesian model of the disambiguation of gravitoinertial force by visual cues. Experimental Brain Research 179:263-90. [MB-C]

MacNeilage, P. R., Banks, M. S., DeAngelis, G. C. \& Angelaki, D. E. (2010) Vestibular heading discrimination and sensitivity to linear acceleration in head and world coordinates. The Journal of Neuroscience 30:9084-94. [MB-C]

Mandel, J. T., Bildstein, K. L., Bohrer, G. \& Winkler, D. W. (2008) Movement ecology of migration in turkey vultures. Proceedings of the National Academy of Sciences USA 105(49):19102-107. doi:10.1073/pnas.0801789105. [AMH]

Marr, D. (1982) Vision. A computational investigation into the human representation and processing of visual information. W.H. Freeman. [DMWP]

Mattingley, J. B., Bradshaw, J. L. \& Phillips, J. G. (1992) Reappraising unilateral neglect. Australian Journal of Psychology 44:163-69. [JGP]

McIntyre, J., Zago, M., Berthoz, A. \& Lacquaniti, F. (2001) Does the brain model Newton's laws? Nature Neuroscience 4:693-4. [MB-C]

McNamara, T. P. (1986) Mental representations of spatial relations. Cognitive Psychology 18:87-121. [HS]

McNamara, T. P. \& Diwadkar, V. (1997) Symmetry and asymmetry of human spatial memory. Cognitive Psychology 34:160-90. [HS]

Merfeld, D. M., Young, L. R., Oman, C. M. \& Shelhamer, M. J. (1993) A multidimensional model of the effect of gravity on the spatial orientation of the monkey. Journal of Vestibular Research 3(2):141-61. [aKJJ]

Merfeld, D. M. \& Zupan, L. H. (2002) Neural processing of gravitoinertial cues in humans. III. Modeling tilt and translation responses. Journal of Neurophysiology 87(2):819-33. [aKJJ]

Messing, R. M. \& Durgin, F. H. (2005) Distance perception and the visual horizon in head-mounted displays. Transactions on Applied Perception 2:234-50. [FHD]

Michaux, N., Pesenti, M., Badets, A., Di Luca, S. \& Andres, M. (2010) Let us redeploy attention to sensorimotor experience. Behavioral and Brain Sciences 33:283-84. [ABa]

Mittelstaedt, H. (1983) A new solution to the problem of the subjective vertical. Naturwissenschaften 70:272-81. [MB-C]

Mittelstaedt, H. (1998) Origin and processing of postural information. Neuroscience and Biobehavioral Reviews 22(4):473-78. [aKJJ]

Moar, I. \& Bower, G. (1983) Inconsistency in spatial knowledge. Memory and Cognition 11:107-13. [HS]

Moghaddam, M., Kaminsky, Y. L., Zahalka, A. \& Bures, J. (1996) Vestibular navigation directed by the slope of terrain. Proceedings of the National Academy of Sciences of the United States of America 93(8):3439-43. [aKJJ]

Montello, D. R. (1991) The measurement of cognitive distance: Methods and construct-validity. Journal of Environmental Psychology 11(2):101-22. [CCC]

Montello, D. R. (2005) Navigation. In: The Cambridge handbook of visuospatial thinking, ed. P. Shah \& A. Miyake, pp. 257-94. Cambridge University Press. $[\mathrm{CH}]$

Montello, D. R. \& Pick, H. L. (1993) Integrating knowledge of vertically aligned largescale spaces. Environment and Behavior 25(3):457-84. [aKJJ, ABe, CCC]

Morishita, H. \& Hensch, T. K. (2008) Critical period revisited: Impact on vision. Current Opinion in Neurobiology 18(1):101-107. [rKJJ]

Moser, E. I., Kropff, E. \& Moser, M. B. (2008) Place cells, grid cells, and the brain's spatial representation system. Annual Review of Neuroscience 31:69-89. $[\mathrm{aKJJ}]$

Moser, E. I. \& Moser, M. B. (2008) A metric for space. Hippocampus 18(12):114256. [aKJJ]

Mueller, M. \& Wehner, R. (2010) Path integration provides a scaffold for landmark learning in desert ants. Current Biology 20:1368-71. [TBdP]

Muller, R. U. \& Stead, M. (1996) Hippocampal place cells connected by Hebbian synapses can solve spatial problems. Hippocampus 6(6):709-19. [FTS]

Nardi, D. \& Bingman, V. P. (2009a) Pigeon (Columba livia) encoding of a goal location: The relative importance of shape geometry and slope information. Journal of Comparative Psychology 123:204-16. [DN]

Nardi, D. \& Bingman, V. P. (2009b) Slope-based encoding of a goal location is unaffected by hippocampal lesions in homing pigeons (Columba livia). Behavioural Brain Research 205(1):322-26. [aKJJ, DN]

Nardi, D., Mauch, R. J., Klimas, D. B. \& Bingman, V. P. (2012) Use of slope and feature cues in pigeon (Columba livia) goal-searching behavior. Journal of Comparative Psychology 126:288-93. [DN] 
Nardi, D., Newcombe, N. S. \& Shipley, T. F. (2011) The world is not flat: Can people reorient using slope? Journal of Experimental Psychology. Learning, Memory and Cognition 37(2):354-67. [aKJJ]

Nardi, D., Nitsch, K. P. \& Bingman, V. P. (2010) Slope-driven goal location behavior in pigeons. Journal of Experimental Psychology. Animal Behavior Processes 36 (4):430-42. [aKJJ, DN]

Nieder, A. \& Miller, E. K. (2004) A parieto-frontal network for visual numerical information in the monkey. Proceedings of the National Academy of Sciences USA 101:7457-62. [ABa]

Nieh, J. C., Contrera, F. A. \& Nogueira-Neto, P. (2003) Pulsed mass recruitment by a stingless bee, Trigona hyalinata. Proceedings of the Royal Society of London, B: Biological Sciences 270(1529):2191-96. [aKJJ]

Nieh, J. C. \& Roubik, D. W. (1998) Potential mechanisms for the communication of height and distance by a stingless bee, Melipona panamica. Behavioral Ecology and Sociobiology 43(6):387-99. [aKJJ, CCC]

Nitz, D. A. (2011) Path shape impacts the extent of CAl pattern recurrence both within and across environments. Journal of Neurophysiology 105:1815-24. [FSa]

Normand, E. \& Boesch, C. (2009) Sophisticated Euclidean maps in forest chimpanzees. Animal Behaviour 77(5):1195-201. doi:10.1016/j. anbehav.2009.01.025. [AMH]

Noser, R. \& Byrne, R. (2007a) Mental maps in chacma baboons (Papio ursinus): Using inter-group encounters as a natural experiment. Animal Cognition 10 (3):331-40. doi: 10.1007/s10071-006-0068-x. [AMH]

Noser, R. \& Byrne, R. (2007b) Travel routes and planning of visits to out-of-sight resources in wild chacma baboons, Papio ursinus. Animal Behaviour 73(2):25766. doi: 10.1016/j.anbehav.2006.04.012. [AMH]

O’Keefe, J. \& Burgess, N. (1996) Geometric determinants of the place fields of hippocampal neurons. Nature 381:425-28. [PAD, FSa]

O'Keefe, J. \& Dostrovsky, J. (1971) The hippocampus as a spatial map. Preliminary evidence from unit activity in the freely-moving rat. Brain Research 34(1):17175. [aKJJ]

O’Keefe, J. \& Nadel, L. (1978) The hippocampus as a cognitive map. Clarendon Press. [aKJJ]

Oman, C. (2007) Spatial orientation and navigation in microgravity. In: Spatial processing in navigation, imagery and perception, ed. F. W. Mast \& L. Jancke. Springer. [aKJJ]

Oman, C. M., Lichtenberg, B. K., Money, K. E. \& McCoy, R. K. (1986) M.I.T./ Canadian vestibular experiments on the Spacelab-1 mission: 4 . Space motion sickness: Symptoms, stimuli, and predictability. Experimental Brain Research $64(2): 316-34 . \quad[\mathrm{aKJJ}]$

Ooi, T. L. \& He, Z. J. (2007) A distance judgment function based on space perception mechanisms: Revisiting Gilinsky's (1951) equation. Psychological Review 114:441-54. [FHD]

Ooi, T. L., Wu, B. \& He, Z. J. (2001) Distance determined by the angular declination below the horizon. Nature 414:197-99. [FHD, AP]

Orban, G. A. (2011) The extraction of 3D shape in the visual system of human and nonhuman primates. Annual Review of Neuroscience 34:361-88. [GAO]

O'Shea R. P. \& Ross, H. E. (2007) Judgments of visually perceived eye level (VPEL) in outdoor scenes: Effects of slope and height. Perception 36:1168-78. [HER]

Pasqualotto, A. \& Proulx, M. J. (2012) The role of visual experience for the neural basis of spatial cognition. Neuroscience and Biobehavioral Reviews 36:1179-87. [AP]

Pasqualotto, A., Spiller, M. J., Jansari, A. S. \& Proulx, M. J. (2013) Visual experience is necessary for the representation of spatial patterns. Behavioural Brain Research 236:175-79. [AP]

Passini, R. \& Proulx, G. (1988) Wayfinding without vision: An experiment with congenitally totally blind people. Environment and Behavior 20:227-52. [AP]

Payne, R. S. (1971) Acoustic location of prey by barn owls (Tyto alba). Journal of Experimental Biology 54:535-73. [FTS]

Paz-Villagrán, V., Lenck-Santini, P.-P., Save, E. \& Poucet, B. (2002) Properties of place cell firing after damage to the visual cortex. European Journal of Neuroscience 16:771-76. [AP]

Pellicano, E., Smith, A. D., Cristino, F., Hood, B. M., Briscoe, J. \& Gilchrist, I. D. (2011) Children with autism are neither systematic nor optimal foragers. Proceedings of the National Academy of Sciences USA 108(1):421-26. [KAL]

Peponis, J., Zimring, C. \& Choi, Y. K. (1990) Finding the building in wayfinding. Environment and Behavior 22(5):555-90. [CH]

Péruch, P., Vercher, J. L. \& Gauthier, G. M. (1995) Acquisition of spatial knowledge through visual exploration of simulated environments. Ecological Psychology $7: 1-20$. [AP]

Phillips, F. \& Layton, O. (2009) The traveling salesman problem in the natural environment. Journal of Vision 9(8):1145. [aKJJ]

Phillips, J. G. \& Ogeil, R. P. (2010) Curved motions in horizontal and vertical orientations. Human Movement Science 29:737-50. [JGP]

Phillips, J. G. Triggs, T. J. \& Meehan, J. W. (2005) Forward/up directional incompatibilities during cursor placement within graphical user interfaces. Ergonomics 48:722-35. [JGP]
Pick, H. L. \& Rieser, J. J. (1982) Children’s cognitive mapping. In: Spatial orientation: Development and physiological bases, ed. M. Potegal, pp. 107-28. Academic Press. [aKJJ]

Pigarev, I. N. \& Levichkina, E. V. (2011) Distance modulated neuronal activity in the cortical visual areas of cats. Experimental Brain Research 214(1):105-11. [rKJJ]

Pinker, S. (2007) The stuff of thought: Language as a window into human nature. Penguin Books. [KJH]

Postma, A., Zuidhoek, S., Noordzij, M. L. \& Kappers, A. M. L. (2007) Differences between early-blind, late-blind, and blindfolded-sighted people in haptic spatial configuration learning and resulting memory traces. Perception 36:125365. $[\mathrm{AP}]$

Poucet, B., Save, E. \& Lenck-Santini, P.-P. (2000) Sensory and memory properties of place cells firing. Reviews in the Neurosciences 11:95-111. [AP]

Pouget, A., Deneve, S., Ducom, J.-C. \& Latham, P. E. (1999) Narrow versus wide tuning curves: What's best for a population code? Neural Computation 11:8590. [SRL]

Pouget, A., Fisher, S. \& Sejnowski, T. J. (1993) Egocentric representation in early vision. Journal of Cognitive Neuroscience 5(2):150-61. [DMK]

Pouget, A. \& Sejnowski, T. J. (1997) Spatial transformations in the parietal cortex using basis functions. Journal of Cognitive Neuroscience 9(2):222-37. [DMK]

Powers, W. T. (1973) Behavior: The control of perception. Aldine. [FHD]

Pravosudov, V. V. \& Clayton, N. S. (2002) A test of the adaptive specialization hypothesis: Population differences in caching, memory, and the hippocampus in black-capped chickadees (Poecile atricapilla). Behavioral Neuroscience 116:515-22. [DN]

Proffitt, D. R., Bhalla, M., Gossweiler, R. \& Midgett, J. (1995) Perceiving geographical slant. Psychonomic Bulletin and Review 2(4):409-28. [aKJJ, FHD, HER]

Proulx, M. J., Brown, D. J., Pasqualotto, A. Neuroscience and Biobehavioral Reviews [http://dx.doi.org/10.1016/j.neubiorev.2012.11.017] Epub ahead of print, [AP]

Proulx, M. J., Brown, D. J., Pasqualotto, A. \& Meijer, P. (2012) Multisensory perceptual learning and sensory substitution. Neuroscience and Biobehavioral Reviews. 2012 Dec 7. pii: S0149-7634(12)00207-2. [AP]

Proulx, M. J., Stoerig, P., Ludowig, E. \& Knoll, I. (2008) Seeing “where” through the ears: Effects of learning-by-doing and long-term sensory deprivation on localization based on image-to-sound substitution. PLoS ONE 3:e1840. [AP]

Quinn, P. C., Polly, J. L., Furer, M. J., Dobson, V. \& Narter, D. B. (2002) Young infants' performance in the object-variation version of the above-below categorization task: A result of perceptual distraction or conceptual limitation? Infancy 3:323-47. [AGD]

Reppert, S. M. (2006) A colorful model of the circadian clock. Cell 124:233-36. [JGP]

Restat, J. D., Steck, S. D., Mochnatzki, H. F. \& Mallot, H. A. (2004) Geographical slant facilitates navigation and orientation in virtual environments. Perception 33 (6):667-87. [aKJJ, SMW]

Riener, C. R., Stefanucci, J. K., Proffitt, D. R. \& Clore, G. (2011) An effect of mood on the perception of geographical slant. Cognition and Emotion 25(1):17482. [aKJJ]

Rieser, J. J., Pick, H. L., Jr., Ashmead, D. \& Garing, A. (1995) Calibration of human locomotion and models of perceptual-motor organization. Journal of Experimental Psychology: Human Perception and Performance 21:480-97. [FHD]

Röder, B., Kusmierek, A., Spence, C. \& Schicke, T. (2007) Developmental vision determines the reference frame for the multisensory control of action. Proceedings of the National Academy of Sciences USA 104:4753-58. [AP]

Rolls, E. T. (1999) Spatial view cells and the representation of place in the primate hippocampus. Hippocampus 9:467-80. [SRL]

Ross, H. E. (1974) Behaviour and perception in strange environments. Allen \& Unwin. [HER]

Ross, H. E. (1994) Scaling the heights. Scottish Mountaineering Club Journal 255 (185):402-10. [HER]

Ross, H. E. (2006) Judgements of frontal slope in nearby outdoor scenes. In: Fechner Day 2006, ed. D. E. Kornbrot, R. M. Msetfi \& A. W. MacRae, pp. 257-62. International Society for Psychophysics. [HER]

Ross, H. E. (2010) Perspective effects in frontal slope perception. In: Fechner Day 2010, ed. A. Bastianelli \& G. Vidotto, pp. 87-92. International Society for Psychophysics. [HER]

Roswarski, T. E. \& Proctor, R. W. (1996) Multiple spatial codes and temporal overlap in choice reaction tasks. Psychological Research 59:196-211. [IB]

Sabra, A. I. (1989) The Optics of Ibn al-Haytham. Books I-III: On direct vision. Vol. 1, trans. A. I. Sabra, pp. 163-66. The Warburg Institute, University of London. [HER]

Sampaio, C. \& Wang, R. F. (2009) Category-based errors and the accessibility of unbiased spatial memories: A retrieval model. Journal of Experimental Psychology: Learning, Memory, and Cognition 35(5):1331-37. [RFW]

Sargolini, F., Fyhn, M., Hafting, T., McNaughton, B. L., Witter, M. P., Moser, M. B. \& Moser, E. I. (2006) Conjunctive representation of position, direction, and velocity in entorhinal cortex. Science 312(5774):758-62. [aKJJ] 
Sato, N., Sakata, H., Tanaka, Y. L. \& Taira, M. (2006) Navigation-associated medial parietal neurons in monkeys. Proceedings of the National Academy of Sciences USA 103:17001-7006. [ABa]

Savardi, U. \& Bianchi, I. (2009) The spatial path to contrariety. In: The perception and cognition of contraries, ed. U. Savardi, pp. 63-92. McGraw-Hill. [IB]

Savardi, U., Bianchi, I. \& Bertamini, M. (2010) Naive prediction of orientation and motion in mirrors. From what we see to what we expect reflections to do. Acta Psychologica 134(1):1-15. doi: 10.1016/J.ACTPSY.2009.11.008. [IB]

Savelli, F. \& Knierim, J. J. (2012) Flexible framing of spatial representations in the hippocampal formation. Poster presented at the Society for Neuroscience Conference, New Orleans, LA, October 13-17, 2012. Poster No. 812.13. [FSa]

Savelli, F., Yoganarasimha, D. \& Knierim, J. J. (2008) Influence of boundary removal on the spatial representations of the medial entorhinal cortex. Hippocampus 18:1270-82. [FSa]

Schaafsma, S. J. \& Duysens, J. (1996) Neurons in the ventral intraparietal area of awake macaque monkey closely resemble neurons in the dorsal part of the medial superior temporal area in their responses to optic flow patterns. Journal of Neurophysiology 76(6):4056-68. [GAO]

Schäfer, M. \& Wehner, R. (1993) Loading does not affect measurement of walking distance in desert ants Cataglyphis fortis. Verhandlungen der Deutschen Zoologischen Gesellschaft 86:270. [aKJJ]

Schnall, S., Harber, K. D., Stefanucci, J. K. \& Proffitt, D. R. (2012) Social support and the perception of geographical slant. Journal of Experimental Social Psychology 44:1246-55. [aKJJ]

Schnitzler, H.-U., Moss, C. F. \& Denzinger, A. (2003) From spatial orientation to food acquisition in echolocating bats. Trends in Ecology and Evolution 18 (8):386-94. [CFM]

Schultheis, H. \& Barkowsky, T. (2011) Casimir: An architecture for mental spatial knowledge processing. Topics in Cognitive Science 3:778-95. [HS]

Schultheis, H., Bertel, S., Barkowsky, T. \& Seifert, I. (2007) The spatial and the visual in mental spatial reasoning: An ill-posed distinction. In: Spatial cognition V: Reasoning, action, interaction (Lecture Notes in Computer Science, vol. 4387), ed. T. Barkowsky, M. Knauff, G. Ligozat \& R. D Montello, pp. 191-209. Springer. [HS]

Schwabe, L. \& Blanke, O. (2008) The vestibular component in out-of-body experiences: A computational approach. Frontiers in Human Neuroscience 2:17. Available at: http://www.frontiersin.org/human_neuroscience/10.3389/ neuro.09.017.2008/abstract [MB-C]

Schwarz, W. \& Keus, I. M. (2004) Moving the eyes along the mental number line: Comparing SNARC effects with saccadic and manual responses. Perception and Psychophysics 66:651-64. [ABa]

Seidl, T. \& Wehner, R. (2008) Walking on inclines: How do desert ants monitor slope and step length. Frontiers in Zoology 5:8. [aKJJ]

Sereno, A. B. \& Lehky, S. R. (2011) Population coding of visual space: Comparison of spatial representations in dorsal and ventral pathways. Frontiers in Computational Neuroscience 4:159. doi:110.3389/fncom.2010.00159. [SRL]

Sereno, I. M., Pitzalis, S. \& Martinez, A. (2001) Mapping of contralateral space in retinotopic coordinates by a parietal cortical area in humans. Science 294:135054. [HS]

Shelton, P. A., Bowers, D. \& Heilman, K. M. (1990) Peripersonal and vertical neglect. Brain 113:191-205. [JGP]

Sherry, D. F. \& Vaccarino, A. L. (1989) Hippocampus and memory for food caches in black-capped chickadees. Behavioral Neuroscience 103:308-18. [DN]

Shinder, M. E. \& Taube, J. S. (2010) Responses of head direction cells in the anterodorsal thalamus during inversion. Society for Neuroscience Abstracts, No. 895.1/FFF6. [Society of Neuroscience Meeting Planner 2010, San Diego, CA, Program No. 895.1.] (Online). [aKJJ]

Si, B., Kropff, E. \& Treves, A. (2012) Grid alignment in entorhinal cortex. Biological Cybernetics 106(8-9):483-506. doi: 10.1007/s00422-012-0513-7. [FSt]

Siegel, J. J., Neunuebel, J. P. \& Knierim, J. J. (2007) Dominance of the proximal coordinate frame in determining the locations of hippocampal place cell activity during navigation. Journal of Neurophysiology 99:60-76. [FSa]

Silder, A., Besier, T. \& Delp, S. L. (2012) Predicting the metabolic cost of incline walking from muscle activity and walking mechanics. Journal of Biomechanics 45(10):1842-49. [FTS]

Sinai, M. J., Ooi, T. L. \& He, Z. J. (1998) Terrain influences the accurate judgement of distance. Nature 395:497-500. [AP]

Skaggs, W. E. \& McNaughton, B. L. (1998) Spatial firing properties of hippocampal CAl populations in an environment containing two visually identical regions. Journal of Neuroscience 18:8455-66. [PAD]

Smith, A. D., Gilchrist, I. D. \& Hood, B. M. (2005) Children's search behaviour in large-scale space: Developmental components of exploration. Perception 34 (10):1221-29. [KAL]

Smith, A. D., Hood, B. M. \& Gilchrist, I. D. (2008) Visual search and foraging compared in a large-scale search task. Cognitive Processing 9(2):121-26. [KAL]
Smith, A. D., Hood, B. M. \& Gilchrist, I. D. (2010) Probabilistic cuing in large-scale environmental search. Journal of Experimental Psychology: Learning, Memory, and Cognition 36(3):605-18. [KAL]

Snyder, L. H., Grieve, K. L., Brotchie, P. \& Andersen, R. A. (1998) Separate bodyand world-referenced representations of visual space in parietal cortex. Nature 394(6696):887-91. [rKJJ]

Soeda, M., Kushiyama, N. \& Ohno, R. (1997) Wayfinding in cases with vertical motion. In: Proceedings of MERA 97: International Conference on Environment-Behavior Studies, ed. T. Takahashi \& Y. Nagasawa, pp. 55964, University of Tokyo and Man-Environment Relations Association. $[\mathrm{CH}]$

Solstad, T., Boccara, C. N., Kropff, E., Moser, M.-B. \& Moser, E. I. (2008) Representation of geometric borders in the entorhinal cortex. Science 322:186568. [FSa]

Spiers, H. J., Jovalekic, A. \& Jeffery, K. J. (2009) The hippocampal place codeMultiple fragments or one big map? Society of Neuroscience Abstracts, No. 101.28. [Society of Neuroscience Meeting Planner 2009, Chicago, IL, Program No. 101.28.] (Online). [PAD]

Spinozzi, G., Lubrano, G. \& Truppa, V. (2004) Categorization of above and below spatial relations by tufted capuchin monkeys (Cebus apella). Journal of Comparative Psychology 118:403-12. [AGD]

Squatrito, S., Raffi, M., Maioli, M. G., Battaglia-Mayer, A. (2001) Visual motion responses of neurons in the caudal area PE of macaque monkeys. Journal of Neuroscience 21(4):RC130. [GAO]

Stackman, R. W. \& Taube, J. S. (1998) Firing properties of rat lateral mammillary single units: Head direction, head pitch, and angular head velocity. Journal of Neuroscience 18(21):9020-37. [aKJJ, HY]

Stackman, R. W., Golob, E. J., Bassett, J. P. \& Taube, J. S. (2003) Passive transport disrupts directional path integration by rat head direction cells. Journal of Neurophysiology 90:2862-74. [PAD]

Stackman, R. W., Tullman, M. L. \& Taube, J. S. (2000) Maintenance of rat head direction cell firing during locomotion in the vertical plane. Journal of Neurophysiology 83(1):393-405. [aKJJ, HY]

Steck, S. D., Mochnatzki, H. F. \& Mallot, H. A. (2003) The role of geographical slant in virtual environment navigation. In: Spatial Cognition III: Routes and navigation, human memory and learning, spatial representation and spatial learning. Lecture Notes in Artificial Intelligence, No. 2685, ed. C. Freksa, W. Brauer, C. Habel, \& K. F. Wender, pp. 62-76. Springer. [aKJJ]

Stefanucci, J. K., Proffitt, D. R., Banton, T. \& Epstein, W. (2005) Distances appear different on hills. Perception and Psychophysics 67(6):1052-60. [aKJJ]

Stefanucci, J. K., Proffitt, D. R., Clore, G. L. \& Parekh, N. (2008) Skating down a steeper slope: Fear influences the perception of geographical slant. Perception 37(2):321-23. [aKJJ]

Stella, F., Si, B., Kropff, E. \& Treves, A. (2013) Grid cells on the ball. Journal of Statistical Mechanics: Theory and Experiment. P03013 doi:10.1088/1742-5468/ 2013/03/P03013. [FSt]

Stevens, A. \& Coupe, P. (1978) Distortions in judged spatial relations. Cognitive Psychology 10:422-37. [HS]

Suddendorf, T. \& Corballis, M. C. (2007) The evolution of foresight: What is mental time travel, and is it unique to humans? Behavioral and Brain Sciences 30:299351. $[\mathrm{ABa}]$

Sueur, C. (2011) A non-Lévy random walk in chacma baboons: What does it mean? PloS ONE 6(1):e16131. doi:10.1371/journal.pone.0016131. [AMH]

Tafforin, C. \& Campan, R. (1994) Ethological experiments on human orientation behavior within a three-dimensional space - in microgravity. Advances in Space Research 14(8):415-18. [aKJJ]

Taira, M., Tsutsui, K. I., Jiang, M., Yara, K. \& Sakata, H. (2000) Parietal neurons represent surface orientation from the gradient of binocular disparity. Journal of Neurophysiology 83(5):3140-46. [GAO]

Takahashi, K., Gu, Y., May, P. J., Newlands, S. D., DeAngelis, G. C. \& Angelaki, D. E. (2007) Multimodal coding of three-dimensional rotation and translation in area MSTd: Comparison of visual and vestibular selectivity. Journal of Neuroscience 27(36):9742-56. [GAO]

Takahashi, T. T., Bala, A. D., Spitzer, M. W., Euston, D. R., Spezio, M. L. \& Keller, C. H. (2003) The synthesis and use of the owl's auditory space map. Biological Cybernetics 89(5):378-87. [FTS]

Tanaka, K., Hikosaka, K., Saito, H., Yukie, M., Fukada, Y. \& Iwai, E. (1986) Analysis of local and wide-field movements in the superior temporal visual areas of the macaque monkey. Journal of Neuroscience 6(1):134-44. [GAO]

Taube, J. S., Wang, S. S., Kim, S. Y. \& Frohardt, R. J. (2013) Updating of the spatial reference frame of head direction cells in response to locomotion in the vertical plane. Journal of Neurophysiology 109(3):873-88. [aKJJ]

Taube, J. S. (2007) The head direction signal: Origins and sensory-motor integration. Annual Review of Neuroscience 30:181-207. [aKJJ]

Taube, J. S. \& Burton, H. L. (1995) Head direction cell activity monitored in a novel environment and during a cue conflict situation. Journal of Neurophysiology 74 (5):1953-71. [aKJJ, PAD] 
Taube, J. S., Muller, R. U. \& Ranck, J. B. (1990a) Head-direction cells recorded from the postsubiculum in freely moving rats. I. Description and quantitative analysis. Journal of Neuroscience 10(2):420-35. [aKJ]

Taube, J. S., Muller, R. U. \& Ranck, J. B. (1990b) Head-direction cells recorded from the postsubiculum in freely moving rats. II. Effects of environmental manipulations. Journal of Neuroscience 10(2):436-47. [aKJJ]

Taube, J. S., Stackman, R. W., Calton, J. L. \& Oman, C. M. (2004) Rat head direction cell responses in zero-gravity parabolic flight. Journal of Neurophysiology 92 (5):2887-997. [aKJJ]

Tavosanis, G. (2012) Dendritic structural plasticity. Developmental Neurobiology 72 (1):73-86. [rKJJ]

Taylor, C. \& Caldwell, S. (1972) Running up and down hills: Some consequences of size. Science 178(4065):1096. [AMH]

Teufel, H. J., Nusseck, H.-G., Beykirch, K. A., Butler, J. S., Kerger, M. \& Bülthoff, H. H. (2007) MPI motion simulator: Development and analysis of a novel motion simulator. In: Proceedings of the AIAA Modeling and Simulation Technologies Conference and Exhibit, Hilton Head, South Carolina AIAA, 2007-6476. Available at: http://kyb.mpg.de/fileadmin/user_upload/files/publications/attachments/Teufel2007_4512\%5B0\%5D.pdf [MB-C]

Theeuwes, J. (1994) Stimulus driven capture and attentional set-Selective search for colour and visual abrupt onsets. Journal of Experimental Psychology: Human Perception and Performance 20(4):799-806. [KAL]

Thibault, G., Pasqualotto, A., Vidal, M., Droulez, J. \& Berthoz, A. (2013) How does horizontal and vertical navigation influence spatial memory of multi-floored environments? Attention, Perception, and Psychophysics 75(1):10-15. doi: 10.3758/s13414-012-0405-x [ABe, CCC, AP, rKJJ]

Thiele, H. \& Winter, Y. (2005) Hierarchical strategy for relocation of food targets in flower bats: Spatial memory versus cue-directed search. Animal Behaviour 69:315-27. [HP]

Thompson, R. F., Mayers, K. S., Robertson, R. T. \& Patterson, C. J. (1970) Number coding in association cortex of the cat. Science 168:271-73. [ABa]

Tlauka, M., Wilson, P. N., Adams, M., Souter, C. \& Young, A. H. (2007) An investigation into vertical bias effects. Spatial Cognition and Computation 7(4):36591. [aKJJ, CH]

Todd, J. T. \& Norman, J. F. (2003) The visual perception of 3-D shape from multiple cues: Are observers capable of perceiving metric structure? Perception and Psychophysics 65(1):31-47. [GAO]

Torrealba, F. \& Valdes, J. L. (2008) The parietal association cortex of the rat. Biological Research 41(4):369-77. [GAO]

Treves, A., Kropff, E. \& Biswas, A. (2005) On the triangular grid of entorhinal place fields. Society for Neuroscience Abstracts, Abstract No. 31:198.11. [FSt]

Tsoar, A., Nathan, R., Bartan, Y., Vyssotski, A., Dell’Omo, G. \& Ulanovsky, N. (2011) Large-scale navigational map in a mammal. Proceedings of the National Academy of Sciences USA 108(37):E718-24. doi: 10.1073/pnas.1107365108. [aKJJ, CFM]

Tversky, B. (1993) Cognitive maps, cognitive collages, and spatial mental models. In: Spatial information theory: A theoretical basis for GIS, ed. A. U. Frank \& I. Campari, pp. 14-24. Springer Verlag. [HS]

Ulanovsky, N. \& Moss, C. F. (2007) Hippocampal cellular and network activity in freely moving echolocating bats. Nature Neuroscience 10(2):224-33. [aKJJ, CFM]

Umiltà, C. \& Nicoletti, R. (1990) Spatial stimulus-response compatibility. In: Stimulus-response compatibility: An integrated perspective, ed. R. W. Proctor \& T. G. Reeve, pp. 89-116. North Holland (Elsevier Science Publishing). [IB]

Umiltà, C., Priftis, K. \& Zorzi, M. (2009) The spatial representation of numbers: Evidence from neglect and pseudoneglect. Experimental Brain Research 192:561-69. [ABa]

Valerio, S., Clark, B. J., Chan, J. H., Frost, C. P., Harris, M. J. \& Taube, J. S. (2010) Directional learning, but no spatial mapping by rats performing a navigational task in an inverted orientation. Neurobiology of Learning and Memory 93 (4):495-505. [aKJJ]

Valero, A. \& Byrne, R. W. (2007) Spider monkey ranging patterns in Mexican subtropical forest: Do travel routes reflect planning? Animal Cognition 10(3):30515. doi:10.1007/s10071-006-0066-z. [AMH]

Vargas, J. P., Petruso, E. J. \& Bingman, V. P. (2004) Hippocampal formation is required for geometric navigation in pigeons. European Journal of Neuroscience 20:1937-44. [DN]

Vecchi, T., Tinti, C. \& Cornoldi, C. (2004) Spatial memory and integration processes in congenital blindness. NeuroReport 15:2787-90. [AP]

Veelaert, P. \& Peremans, H. (1999) Flexibility maps: A formalisation of navigation behaviours. Robotics and Autonomous Systems 27:151-69. [HP]

Vidal, M., Amorim, M. A. \& Berthoz, A. (2004) Navigating in a virtual threedimensional maze: How do egocentric and allocentric reference frames interact? Brain Research. Cognitive Brain Research 19(3):244-58. [aKJJ, AP]

Vidal, M., Lipshits, M., McIntyre, J. \& Berthoz, A. (2003) Gravity and spatial orientation in virtual 3D-mazes. Journal of Vestibular Research 13:273-86. [AP]

von der Malsburg, C. (1973) Self-organization of orientation sensitive cells in the striate cortex. Kybernetik 14:85-100. [DMWP]
Wade, M. G. \& Jones, G. (1997) The role of vision and spatial orientation in the maintenance of posture. Physical Therapy 77:619-28. [MBC]

Wagner, M. (1985) The metric of visual space. Perception and Psychophysics 38:483-95. [FHD]

Wall, M. B., \& Smith, A. T. (2008) The representation of egomotion in the human brain. Current Biology 18(3):191-94. [GAO]

Walls, M. L. \& Layne, J. E. (2009) Fiddler crabs accurately measure two-dimensional distance over three-dimensional terrain. The Journal of Experimental Biology 212(Pt. 20):3236-40. [aKJJ]

Walsh, V. (2003) A theory of magnitude: Common cortical metrics of time, space, and quantity. Trends in Cognitive Sciences 7:483-88. [ABa]

Wang, R. F. (2012) Theories of spatial representations and reference frames: What can configuration errors tell us? Psychonomic Bulletin and Review 19(4):575-87. [RFW]

Wang, R. F. (in press). Human four-dimensional spatial judgments of hypervolume. Spatial Cognition \& Computation: An Interdisciplinary Journal. [RFW]

Wang, R. F. \& Brockmole, J. R. (2003) Human navigation in nested environments. Journal of Experimental Psychology: Learning, Memory, and Cognition 29:398404. $[\mathrm{RFW}]$

Wang, R. F. \& Cutting, J. E. (1999) A probabilistic model for recovering camera translation. Computer Vision and Image Understanding 76:205-12. [RFW]

Wang, R. F. \& Spelke, E. S. (2000) Updating egocentric representations in human navigation. Cognition 77:215-50. [RFW]

Wang, R. F. \& Spelke, E. S. (2002) Human spatial representation: Insights from animals. Trends in Cognitive Sciences 6(9):376. [aKJJ]

Wang, R. F. \& Spelke, E. S. (2003) Comparative approaches to human navigation. In: The neurobiology of spatial behaviour, ed. K. Jeffery, pp. 119-43. Oxford University Press. [RFW]

Webber, D. M., Aitken, J. P. \& O’Dor, R. K. (2000) Costs of locomotion and vertic dynamics of cephalopods and fish. Physiological and Biochemical Zoology 73 (6):651-62. [aKJJ]

Wehner, R. (2003) Desert ant navigation: How miniature brains solve complex tasks Journal of Comparative Physiology A: Neuroethology, Sensory, Neural, and Behavioral Physiology 189:579-88. [aKJJ]

Wehner, R., Boyer, M., Loertscher, F., Sommer, S. \& Menzl, U. (2006) Ant navigation: One-way routes rather than maps. Current Biology 16:75-79. [SZ]

Wehner, R. \& Menzel, R. (1990) Do insects have cognitive maps? Annual Review of Neuroscience 13:403-14. [SZ]

Weisberg, S. M., Brakoniecki, E. \& Newcombe, N. S. (2012) The other side of the mountain: Slope as a cue in navigation. Paper presented at the 5 th Internationa Conference on Spatial Cognition, Rome, Italy, September 4-8, 2012. [SMW]

Weisberg, S. M., Nardi, D. N., Newcombe, N. S. \& Shipley, T. F. (2012) Sensing the slopes: Sensory modality effects on a goal location task. Poster presented at the 53rd Annual Meeting of the Psychonomics Society, Minneapolis, MN, November 15-18, 2012. [SMW]

Weisman, J. (1981) Evaluating architectural legibility: Way-finding in the built environment. Environment and Behavior 13(2):189-204. [CH]

Whitlock, J. R. \& Derdikman, D. (2012) Head direction maps remain stable despite grid map fragmentation. Frontiers in Neural Circuits 6, article 9. doi: 10.3389/ fncir.2012.00009. [PAD]

Whitlock, J. R., Sutherland, R. J., Witter, M. P., Moser, M.-B. \& Moser, E. I. (2008) Navigating from hippocampus to parietal cortex. Proceedings of the National Academy of Sciences USA 105(39):14755-62. [GAO]

Wiener, J. M., Hölscher, C., Büchner, S. \& Konieczny, L. (2012) Gaze behaviour during space perception and spatial decision making. Psychological Research 76 (6):713-29. [CH]

Wills, T. J., Cacucci, F., Burgess, N. \& O'Keefe, J. (2010) Development of the hippocampal cognitive map in preweanling rats. Science 328(5985):1573-76. [rKJ]

Wilson, P., Foreman, N., Stanton, D. \& Duffy, H. (2004) Memory for targets in a multilevel simulated environment: Evidence for vertical asymmetry in spatial memory. Memory and Cognition 32(2):283-97. [aKJJ, RFW]

Winter, Y. \& Stich, K. P. (2005) Foraging in a complex naturalistic environment: Capacity of spatial working memory in flower bats. Journal of Experimental Biology 208:539-48. [HP]

Winter, Y., von Merten, S. \& Kleindienst, H.-U. (2004) Visual landmark orientation by flying bats at a large-scale touch and walk screen for bats, birds, and rodents. Journal of Neuroscience Methods 141:283-90. [HP]

Wintergerst, S. \& Ronacher, B. (2012) Discrimination of inclined path segments by the desert ant Cataglyphis fortis. Journal of Comparative Physiology A: Neuroethology, Sensory, Neural, and Behavioral Physiology 198(5):363-73. [aKJJ]

Wohlgemuth, S., Ronacher, B. \& Wehner, R. (2001) Ant odometry in the third dimension. Nature 411(6839):795-98. [aKJJ]

Worringham, C. J. \& Beringer, D. B. (1989) Operator orientation and compatibility in visual-motor task performance. Ergonomics 32:387-99. [JGP]

Wray, M. K., Klein, B. A., Mattila, H. R. \& Seeley, T. D. (2008) Honeybees do not reject dances for "implausible" locations: Reconsidering the evidence for cognitive maps in insects. Animal Behaviour 76:261-69. [SZ] 


\section{References/Jeffery et al.: Navigating in a three-dimensional world}

Wyeth, G. \& Milford, M. J. (2009) Spatial cognition for robots: Robot navigation from biological inspiration. IEEE Robotics and Automation Magazine 16(3):2432. $[\mathrm{HP}]$

Wystrach, A., Schwarz, S., Schultheiss, P., Beugnon, G. \& Cheng, K. (2011) Views, landmarks, and routes: How do desert ants negotiate an obstacle course? Journal of Comparative Physiology A 197:167-79. [SZ]

Yang, Z. \& Purves, D. (2003) A statistical explanation of visual space. Nature Neuroscience 6(6):632-40. [HER]

Yantis, S. \& Jonides, J. (1984) Abrupt visual onsets and selective attention: Evidence from visual search. Journal of Experimental Psychology: Human Perception and Performance 10(5):601-21. [KAL]

Yartsev, M. M. \& Ulanovsky, N. (2013) Representation of threedimensional space in the hippocampus of flying bats. Science 340:367-72. [CFM, FSa]
Yartsev, M. M., Witter, M. P. \& Ulanovsky, N. (2011) Grid cells without theta oscillations in the entorhinal cortex of bats. Nature 479(7371):103-107. doi: 10.1038/nature10583. [CFM]

Young, L. R., Oman, C. M., Watt, D. G., Money, K. E. \& Lichtenberg, B. K. (1984) Spatial orientation in weightlessness and readaptation to earth's gravity. Science 225(4658):205-208. [aKJJ]

Zhang, K. \& Sejnowski, T. J. (1999) Neural tuning: To broaden or to sharpen? Neural Computation 11:75-84. [SRL]

Zhu, H., Sauman, I., Yuan, Q., Casselman, A., Emery-Le, M., Emery, P. \& Reppert, S. M. (2008) Cryptochromes define a novel circadian clock mechanism in Monarch butterflies that may underlie sun compass navigation. PLoS Biology 6:138-55. [JGP]

Zugaro, M. B., Arleo, A., Berthoz, A. \& Wiener, S. I. (2003) Rapid spatial reorientation and head direction cells. Journal of Neuroscience 23(8):3478-82. [aKJJ] 